\title{
Understanding factors affecting postoperative quality of life
}

Citation for published version (APA):

Theunissen, M. (2017). Understanding factors affecting postoperative quality of life. [Doctoral Thesis, Maastricht University]. Datawyse / Universitaire Pers Maastricht. https://doi.org/10.26481/dis.20170607mt

Document status and date:

Published: 01/01/2017

DOI:

$10.26481 /$ dis.20170607mt

Document Version:

Publisher's PDF, also known as Version of record

\section{Please check the document version of this publication:}

- A submitted manuscript is the version of the article upon submission and before peer-review. There can be important differences between the submitted version and the official published version of record.

People interested in the research are advised to contact the author for the final version of the publication, or visit the DOI to the publisher's website.

- The final author version and the galley proof are versions of the publication after peer review.

- The final published version features the final layout of the paper including the volume, issue and page numbers.

Link to publication

\footnotetext{
General rights rights.

- You may freely distribute the URL identifying the publication in the public portal. please follow below link for the End User Agreement:

www.umlib.nl/taverne-license

Take down policy

If you believe that this document breaches copyright please contact us at:

repository@maastrichtuniversity.nl

providing details and we will investigate your claim.
}

Copyright and moral rights for the publications made accessible in the public portal are retained by the authors and/or other copyright owners and it is a condition of accessing publications that users recognise and abide by the legal requirements associated with these

- Users may download and print one copy of any publication from the public portal for the purpose of private study or research.

- You may not further distribute the material or use it for any profit-making activity or commercial gain

If the publication is distributed under the terms of Article $25 \mathrm{fa}$ of the Dutch Copyright Act, indicated by the "Taverne" license above, 


\section{Understanding factors affecting postoperative Quality of Life}

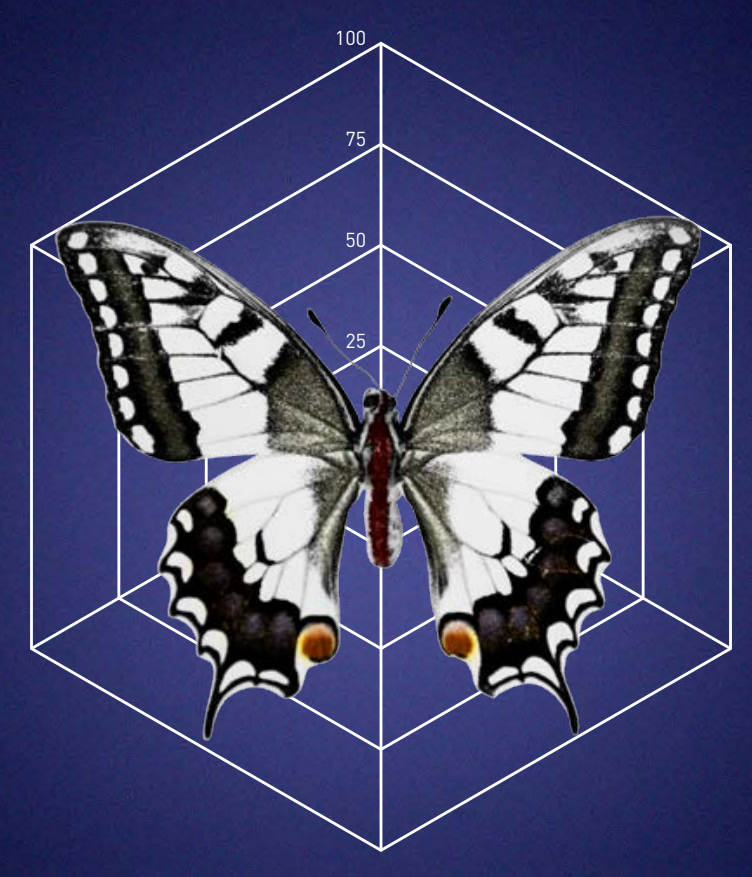

Maurice Theunissen 
Cover photo: Mitchell Giebels | mitchellgiebels.com Prologue: Pieter Theunissen

(C) copyright Maurice Theunissen, Maastricht 2017

Printing: Datawyse | Universitaire Pers Maastricht

ISBN 9789461597014

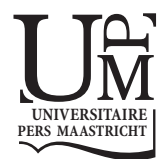




\title{
Understanding factors affecting postoperative Quality of Life
}

\author{
Proefschrift \\ ter verkrijging van de graad van doctor aan de Universiteit Maastricht, \\ op gezag van de Rector Magnificus, Prof. dr. Rianne M. Letschert, \\ volgens het besluit van het College van Decanen, \\ in het openbaar te verdedigen \\ op woensdag 7 juni 2017 om 10:00 uur \\ door
}

Henricus Mauritius Servatius (Maurice) Theunissen 


\section{Promotores}

Prof. dr. M.L. Peters

Prof. dr. M.A.E. Marcus

\section{Copromotor}

Dr. H-F. Gramke

\section{Beoordelingscommissie}

Prof. dr. E.A.J. Joosten (voorzitter)

Prof. dr. C. van Heugten

Dr. J.A.F. Huirne (VUMC Amsterdam)

Prof. dr. C.J. Kalkman (UMC Utrecht)

Dr. S.M.J. van Kuijk

This thesis was supported by an Innovative Research Grant (VICI), received by M.L.

Peters from the Netherlands Organization for Scientific Research (Grant \# 453-07-005) 


\section{Proloog}

I

Snel duwt men mijn ledikant met ronde hoeken, Met strakke en professionele precisie,

Door gangen waarin ik alleen hysterie zie;

De verpleegsters waren knapper in de boeken.

De bittere smaak van synthetische geuren

Kan ik door te ademen rustig vergeten.

In mistige hemelen meen ik te weten:

Gemaskerde engelen staan te gebeuren.

II

Instrumenten liggen scherp gestemd te blinken.

De maestro tikt de maten af in mijn boezem.

Dan laat het orkest, terwijl ik dwaal in droesem

De verre symfonieën groots weerklinken.

Het is donker, traag keert de vervreemding terug.

Terwijl valse honden blaffen in de stegen,

Nadert een zeurende pijn als helse zegen.

Ik lig verstijfd in vieze plassen op mijn rug.

III

In het ochtendlicht voelen mijn lakens als satijn.

Ik kan, met benen licht als papieren vleugels,

Door groene velden draven met losse teugels.

Het had allemaal stukken erger kunnen zijn. 



\section{Content}

Proloog

Chapter 1 General introduction

Chapter 2 Preoperative anxiety and catastrophizing: a systematic review and meta-analysis of the association with chronic postsurgical pain

Chapter 3 Validation of the Surgical Fear Questionnaire in adult patients waiting for elective surgery

Chapter 4 Validity and time course of surgical fear as measured with the Surgical Fear Questionnaire in patients undergoing cataract surgery

Chapter 5 Recovery 3 and 12 months after hysterectomy: epidemiology and predictors of chronic pain, physical functioning, and global surgical recovery

Chapter 6 Prevalence and predictors of depression and well-being after hysterectomy: an observational study

Chapter 7 General discussion

Valorization

Summary

Samenvatting

Dankwoord

Curriculum Vitae 215

List of publications 

Chapter

1

General introduction

Best care...Always! Dixit: Institute for Healthcare Improvement 



\section{POSTOPERATIVE QUALITY OF LIFE}

Obviously, when people undergo curative surgery, their aim is to get better again. Sometimes there is a price to be paid for this in terms of quality of life, depending on the type of surgery. This should be taken into account when making the decision to perform a certain surgical procedure. Quality of life is influenced by many factors and therefore it is very hard to find an adequate evaluation tool for it. When, in the nineties of the last century, the World Health Organization (WHO) developed a questionnaire for the measurement of quality of life, they provided the following definition of quality of life: "individuals' perception of their position in life in the context of the culture and value systems in which they live and in relation to their goals, expectations, standards and concerns. It is a broad ranging concept affected in a complex way by the persons' physical health, psychological state, level of independence, social relationships and their relationship to salient features of their environment." The aim of the WHO was to create an instrument that would enable measurement of quality of life worldwide, across different cultures. Six domains were established: physical, psychological, level of independence, social relationships, environment, and spirituality/religion/personal beliefs. ${ }^{1,}$ ${ }^{2}$ Since the introduction of the concept, quality of life has gained major importance in studies evaluating the effects of surgery. However, depending on the study aim, the focus of measurements often directs towards health-related quality of life, to enable a more detailed evaluation of the surgery. In surgical terms this is denoted by the term postoperative recovery, where postoperative health-related quality of life is compared with preoperative status of health-related quality of life.

Similar to quality of life, postoperative recovery has multiple dimensions and can be considered from many viewpoints such as patient's view, health care workers view, time stages like early-, intermediate-, and long-term recovery, domains of recovery, or assessment of recovery. A concept analysis performed on postoperative recovery resulted in the following definition: "Postoperative recovery is an energy-requiring process of returning to normality and wholeness as defined by comparative standards, achieved by regaining control over physical, psychological, social, and habitual functions, which results in returning to preoperative levels of independence/dependence in activities of daily living and an optimum level of psychological well-being". ${ }^{3}$ Another index of postoperative recovery is cognitive functioning. Although it might be considered part of psychological recovery, psychological recovery often focusses on the more emotional aspects like well-being, depression, or anxiety. However, assessment of social and habitual recovery may indirectly reflect aspects of cognitive function as memory function, intelligence scale, concentration, or language abilities. 


\section{Assessment of postoperative recovery}

For the measurement of recovery many instruments are available ranging from general instruments to targeted instruments focussing on discharge criteria or surgery specific outcomes. A generic one-item scale for self-reported assessment of postoperative recovery is the global surgical recovery (GSR) index. The GSR assesses recovery on a scale from $0 \%$ (no recovery) to 100\% (back to usual health before becoming ill and having surgery). An advantage of this tool is that the patients themselves decide which aspects of recovery are most relevant and thus mirrored in their recovery score. ${ }^{4}$ However, despite many publications on the assessment of surgical recovery, there is no consensus yet about a generally accepted and validated assessment package for the evaluation of surgical recovery. ${ }^{5}$

In 2013 VanDenKerkhof et al. presented a framework with core predictor and outcome domains for chronic postsurgical pain (CPSP) in epidemiological studies, based on the IMMPACT recommendations. Four outcome domains are suggested: pain, physical functioning, psychological functioning, and global ratings of outcome. ${ }^{6}, 7$ Moreover, outcome measures within each domain are suggested. Using these domains and outcome measures, this thesis will consider postoperative quality of life by studying pain, physical and psychological functioning, and global self-perceived recovery. Predictors of these outcomes, will be assessed, and special attention will be paid to the role of surgical fear.

\section{Chronic postsurgical pain}

It was after the disclosure of the results of a survey among British pain centers, revealing that $20 \%$ of the visiting patients suffered from postsurgical pain, that the issue of CPSP really became apparent. ${ }^{8}$ Nowadays CPSP is an important mark of postoperative long-term recovery since the burden of pain on quality of life and society is substantial. Many studies across different parts of the world prove that people with pain, compared to people without pain, have a significantly poorer physical and mental health status, and also show higher levels of absenteeism, work impairment, healthcare resource utilization, emergency room visits, and hospitalization. ${ }^{9-13}$ As a result, all efforts leading to further reduction of postoperative pain are of utmost importance.

A generally accepted definition of CPSP is given by Macrae and consists of four criteria: 1) the pain developed after surgery, 2) has a duration of at least two months, 3) other causes for the pain are excluded, and 4) continuation of a pre-existing problem as potential cause should be excluded. ${ }^{14}$ Recently an update of this definition was proposed. ${ }^{15}$ The most important changes refer to the development of CPSP (expanded with "or increases in intensity after the surgical procedure"), pain duration (adapted to "the pain duration should be at least 3-6 months"), and the location of pain (related to the surgical field, or projected or referred pain). 
Differences in the type of surgery, actual follow-up period, used cut-offs for pain intensity, and the use of composite outcome measures result in a wide range of reported incidence/prevalence of CPSP. For example, reported rates of chronic postoperative pain after ophthalmic surgery are $<5 \%,{ }^{16}$ after hernia repair $5-30 \%,{ }^{17}$ after hysterectomy $5-32 \%{ }^{18}$ after breast cancer surgery $25-50 \%,{ }^{19}$ after thoracotomy $47-57 \%{ }^{20}$ Despite their heterogeneity, these numbers underline the potential impact of further enhancements in postoperative pain prevention.

The etiology of chronic postoperative pain is complex. Pre-, per-, and postoperative factors are probably involved. ${ }^{21,22}$ Potential preoperative factors comprise pre-existing pain, psychosocial factors like surgical fear or pain catastrophizing, and biological factors like sex, age, co-morbidity, and genetics. A peroperative factor is type of incision/surgery which causes inflammatory and nociceptive responses and has the risk of long term nerve damage. Also type of anesthesia and drug use, which for example may be the cause of opioid-induced hyperalgesia, and long duration of surgery are potential factors. A major postoperative factor appears to be acute pain, and depending on the underlying disease, postoperative radiotherapy and chemotherapy may cause additional cell damage with pain as result. Surgical pain can be nociceptive, neuropathic, inflammatory, or visceral. At present, in the evaluation of chronification of postoperative pain, nerve damage and the mechanisms of peripheral and central sensitization are considered the most important.

In the case of chronic postoperative pain the postoperative pain continues after normal wound healing has been established and the original cause of pain has disappeared. The pain manifests itself in two ways. The first is by a lower pain threshold, leading to pain experience of stimuli that were not painful before, called allodynia. The word allodynia means a painful response to a normally innocuous stimulus. The second way is by a higher pain sensibility, which means an increased response to a painful stimulus. In the case of peripheral sensitization, the peripheral nociceptors are in a state of higher excitation, resulting in prolonged and increased responses to pain stimuli or allodynia. Peripheral sensitization leads to increased pain sensitivity in the region of the injury, called primary hyperalgesia. In the case of central sensitization regions of the central nerve system involved in pain processing, in the spinal cord, have evolved to a state of permanent hyperexcitation, leading to allodynia, hyperalgesia, and secondary hyperalgesia, an expansion of hyperalgesia to non-injured areas remote from the initial wound. Besides the spinal cord, also other regions of the central nerve system are associated with the process of central sensitization, such as the brain stem and the somatosensory cortex. Importantly, endogeneous pain modulation can facilitate or inhibit nociceptive spinal input and as such, enhance or dampen pain sensation, including the effects of central sensitization. This mechanism of top-down control, originates from the brain stem. Biopsychosocial factors such as stress, fear, or catastrophizing, or different modes of surgery such as minimally invasive surgery, and different anesthesia tech- 
niques such as spinal analgesia or use of multimodal preventive analgesia, can modulate processes described above either in a positive or negative way. ${ }^{22-27}$

In addition to peripheral and central sensitization, nerve damage could be an underlying cause of persistent postsurgical pain. Nerve damage is associated with the occurrence of neuropathic pain. Neuropathic pain is defined as "pain arising as a direct consequence of a lesion or disease affecting the somatosensory system." ${ }^{28}$ In the case of postoperative pain this may occur as a result of surgical nerve lesion, surgical retractor placement, positioning of the patient, or nerve entrapment. ${ }^{29-31}$ In the pathway from neuropathy to pain, the root of the matter is "ectopia". Under certain circumstances after nerve injury, secondary changes in the afferent sensory neurons occur. As a result a considerable part of these neurons begin to fire spontaneously. This ectopic response can occur within hours or days after the injury. Several factors may enhance ectopic reactivity, such as mechanical stimulation, temperature changes, hypoxia, or circulating catecholamines. Until now it is not clarified what circumstances are relevant in the etiology of painful ectopia, since not all nerve injuries lead to neuropathic pain. However, altered gene expression of injured neurons in the dorsal root ganglion plays a role, but it is not clear yet which of the thousands of up- or down-regulated genes exactly are relevant. Furthermore alterations in the trafficking of the signalling molecules and of the channel kinetics and receptor molecules lead to higher excitability of the system. ${ }^{32}$ In fact, surgical nerve injury may also trigger the process of central sensitization, leading via neuronal plasticity to a state of pain hypersensivity as described earlier. However, the etiology is different: ectopic reactivity versus hyperactive nociceptors.

\section{Physical functioning}

Physical functioning is an important measure of health-related quality of life. It reflects physical condition as such, but high levels of physical function also have a positive impact on many aspects of physical and psychosocial health status and mortality, as is consistently proven across many studies in various populations. ${ }^{33-37}$ Thus, it is recommended that in relation to perioperative assessments physical functioning is measured. To improve physical functioning after surgery many intervention studies are performed, applying pre- or postoperative physical exercises. For preoperative interventions also the term prehabilitation is used, analogous to postoperative rehabilitation. Prehabilitation aims at "improving an individual's functional capacity through increased physical activity before an anticipated procedure." ${ }^{38}$ Using a dedicated programme the body can be prepared, first to undergo a stressful situation and second, to a faster return to preoperative level of functioning. However, despite many publications, the number of studies included in reviews and meta-analyses is often relatively low and the results on such interventions are still conflicting, not in the last place because of heterogeneity in study design. ${ }^{39-41}$ This is also the case for postoperative interventions. ${ }^{42}$ Nonetheless, physical optimization has become more and more important and the future direction 
might best be summarized by the publication of Hoogeboom et al. ${ }^{43}$ in which they propose perioperative exercise therapy for elective surgery patients at increased risk of postoperative complications, and further study on the establishment of groups at risk and effective interventions. Therefore, additional information on physical function before and after surgery, and its related factors, will probably enable further unraveling of the involved mechanisms. These insights might be helpful in optimizing patient care in the future.

\section{Depression and well-being}

Both depression and well-being are important aspects in the evaluation of postoperative psychological quality of life. Preoperative complaints as indication for surgery or the burden of a new diagnosis can cause increased feelings of depression or reduced wellbeing. Depending on the results of surgery these feelings might persist postoperatively or, if not present before, occur after surgery, or preferably, disappear after surgery. Besides surgical stress, feelings of depression can be triggered by several other factors. Genetics, recent or early-life stress events like childhood maltreatment or a divorce, a person's psychosocial situation, inflammation, and insomnia, are factors that may affect depression. ${ }^{44-50}$ Besides the burden of depression on itself, the presence of depression in surgical patients may also be a risk factor for other postoperative mobidity. ${ }^{51}$

Depression is considered a measure of ill-being, like anxiety, negative affect, and anger. On the other hand, well-being, the state of being healthy, happy, or prosperous ${ }^{52}$ reflects a positively oriented dimension of psychological functioning. In other words, well-being can be seen as more than just the absence of psychopathology. ${ }^{53}$ Because the absence of ill-being not automatically implies the presence of well-being, assessing both ill- and well-being for evaluation of postoperative quality of life is preferred. However, the research on the mutual independence of the concepts ill-being and well-being is inconclusive and ongoing, as reflected in the paper of Franz et al. ${ }^{54}$ Combining the results of their twin study with the existing knowledge from publications on genetic, environmental, and biological parameters, they conclude that there is evidence "that illand well-being are not simply at opposite ends of a continuum, neither are they totally independent." Factors associated with higher levels of well-being are associated with personality characteristics: a positive spirit, optimism, and a tendency to not to ruminate too much about negative experiences. Also social factors are involved and comprise living in an economically flourishing region, having close friends or family to discuss private matters, and having the ability to pursue relevant goals. ${ }^{55}$

Similar to physical $(p)$ rehabilitation programmes, also psychological ( $p$ )rehabilitation programmes exist, to improve the pre- and postoperative status. Several types of interventions have been studied, such as stress management by a psychologist, ${ }^{56}$ guided imagery, ${ }^{56,57}$ relaxation, ${ }^{56,57}$ a teaching programme with patient instruction, ${ }^{56,58}$ a psychotherapeutic intervention combined with an interview, ${ }^{56}$ nurse counseling, $^{59}$ and 
hypnosis. ${ }^{57,60}$ Some studies revealed partial or even strong evidence for postoperative improvements, however not all studies were positive and in some of the studies the quality of evidence was limited.

We conclude that with regard to depression and well-being, similar to physical functioning and pain, a considerable amount of research has been performed. However, for many interventions and patient groups the benefit is still unclear and additional perioperative information is necessary.

\section{PREDICTORS OF POSTSURGICAL PAIN AND OTHER ASPECTS OF POSTOPERATIVE QUALITY OF LIFE}

As illustrated in the previous paragraph by the examples of studies on the association between physical/psychological prehabilitation and postoperative outcome, and about the etiology of postoperative pain, clinicians and researchers are constantly trying to unravel the potential associations between preoperative patient status, peroperative interventions, and postoperative outcome. Common goal is to predict who is at risk of impaired outcome and what preventive measures could be implemented for those groups at risk. In the last decades progression has been made and this has yielded relevant improvements in perioperative care. In the next paragraph examples are given of several predictor studies. Then a short introduction on surgical fear is presented, the topic of the chapters 2,3 , and 4.

\section{Predictor studies}

There are many studies on predictors of postoperative pain or other outcomes. Pain is one of the most intensively investigated parameters. Early studies were relevant for signalling medical issues and gave direction to future study and treatment options. However, many studies consisted of small study populations, ${ }^{61}$ used a retrospective design, ${ }^{62}$ or performed only limited correction for covariates based on simple stratified analyses. ${ }^{61,63}$ Other studies only assessed biomedical predictors without considering psychological aspects. ${ }^{64}$ In later studies the quality of evidence improved remarkably as a result of prospective designs in combination with multivariate analyses using comprehensive sets of predictors and larger study samples. For example, in 2007 our study group performed a study to assess predictors of CPSP, functional limitations, global surgical recovery, and quality of life after various types of surgery. ${ }^{65}$ Using a prospective design data of 625 clinical patients were analysed, duration of follow-up was six months. Among several medical, surgical, and psychosocial predictors were pre- and postoperative pain and surgical fear. A consecutive study was performed on another sample of 908 day surgery patients. Predictors of chronic pain and global surgical recovery were assessed and similar predictors were found. ${ }^{66}$ Other studies have devel- 
oped multifactorial prediction rules e.g. for early postoperative pain, ${ }^{67,68}$ or persistent pain after breast surgery. ${ }^{69}$ Altogether, these more recent studies have led to increased insight in predictors and processes involved in chronic pain, ${ }^{22}$ and postoperative healthrelated quality of life. ${ }^{70,71}$

However, although the more recent studies were generally well performed, heterogeneity in types of surgery still impair the strength of conclusions. Therefore, future studies investigating a single type of surgery on a more homogeneous population are still of additional value. In this regard hysterectomy patients are a suitable population. Hysterectomy is a frequently performed operation: in the Netherlands in 201011697 hysterectomies were carried out in women aged between 20 and 65 years. ${ }^{72}$ In this thesis a comprehensive study on hysterectomy patients is described.

\section{Surgical fear}

The first part of this thesis will focus on an important psychological predictor for surgical outcome, preoperative anxiety or fear. Attention for anxiety and fear in the preoperative period dates back to decades ago or even earlier. Publications of Sheldon "The false fear of disc surgery" (1949), ${ }^{73}$ and Walsh in Psychotherapy Chapter 2 "Mental influence before operation" (1912) ${ }^{74}$ are early examples of scientific interest. In more general terms, in ancient times Aristotle already wrote about the effect of music as a medicine creating an emotion of relaxation, and creating a pleasure from which we obtain rest, ${ }^{75}$ implying that negative emotions might affect health negatively. Recently, several review articles clustered the existing insights on possible associations between preoperative anxiety or fear and postoperative outcome. ${ }^{76-78}$

In the case of preoperative anxiety or surgical fear, more state-like anxiety or fear might play a role, whereas in many studies on surgical outcome for the assessment of preoperative anxiety or fear, general instruments were used. Even if state anxiety was assessed, most of the used instruments are not designed to assess specific surgeryrelated anxiety or fear. Because the existing psychological and surgical literature reveals a potentially important role for anxiety and fear in the preoperative stage, in this thesis two aspects are subjected to further study.

The first aspect is the assessment of the association between preoperative anxiety or pain catastrophizing and CPSP. As already mentioned, psychological mechanisms play a role in pain sensitivity. And currently, for the association between preoperative anxiety and acute postoperative pain convincing evidence exists. However, for chronic postoperative pain the association with preoperative anxiety is less clear until now. Since many new studies emerged in the last years, we performed a literature review including a meta-analysis, to assess the actual state of evidence with regard to the association between chronic postoperative pain and preoperative anxiety.

The second aspect we seek to answer in this thesis is the validation of the Surgical Fear Questionnaire. The Surgical Fear Questionnaire was developed in 2004 to assess 
specifically the fear of surgery. We describe the development of the instrument, the reliability, and the construct, content, convergent, and predictive validity. Data of five observational studies applying the Surgical Fear Questionnaire were used, one study on mixed in-hospital surgery patients, two studies on mixed day-surgery patients, and two studies on hysterectomy patients. Because these studies only assessed surgical fear at one moment before surgery, further study was performed in cataract patients. The aim of this study was to assess the course of surgical fear in the week before surgery and to further validate the questionnaire. In addition to self-report questionnaires, salivary cortisol and alpha amylase samples were collected as biomarkers of stress.

\section{AIM AND RESEARCH QUESTIONS}

Knowledge on predictors of postoperative quality of life facilitates targetted physical and psychosocial prehabilitation, which, in combination with dedicated surgical and anesthesiological techniques, and optimized postoperative care can lead to higher quality of life after surgery. In this thesis we try to seek answers for several research questions. In chapter two, data of 29 prospective studies on musculoskeletal surgery and various other types of surgery are described. By means of a literature review and metaanalysis we provide an answer to research question 1:

What is the current level of evidence concerning the hypothesis that high levels of preoperative anxiety or pain catastrophizing are associated with an increased risk of chronic postsurgical pain?

As chapter two reveals, a diversity of instruments exist to assess anxiety and fear. For the assessment of surgical fear however, only a limited number of instruments is available and existing instruments are either limited in scope, or aiming at specific patient groups. So there is need for an instrument which is suitable for a broad scope of patients and surgery types, and which is easy to obtain in view of a general clinical use in the future. Therefore the Surgical Fear Questionnaire was developed, as an instrument dedicated to detect fear of surgery in adult patients across different types of surgery. In chapter three, using data of five different studies we seek to answer research question 2:

Is the Surgical Fear Questionnaire a valid and reliable instrument for the assessment of surgical fear?

The validation process of the Surgical Fear Questionnaire as described in chapter three shows that in all studies that provided data, the Surgical Fear Questionnaire was assessed only once. As a result, the sensitivity to differences in fear level over time is unknown. Also the convergent validity with biological indices of stress is unknown. In order to solve this problem, a study was performed in cataract surgery patients, where we assessed the course of surgical fear in the week before cataract surgery and simultane- 
ously collected samples of salivary cortisol and alpha-amylase. In chapter four we describe our findings, to answer research question 3 :

What is the sensitivity of the Surgical Fear Questionnaire with regard to the detection of potential differences in surgical fear across time, in the week before cataract surgery, and is there an association between the level of surgical fear assessed by the Surgical Fear Questionnaire, and salivary cortisol and salivary alpha amylase levels?

For the study on predictors of postoperative quality of life a multicenter observational study was performed on elective hysterectomy patients with a benign indication. Chapter five describes predictors of pain, physical function, and global surgical recovery, whereas in chapter six psychological aspects of postoperative quality of life are addressed. Moreover, epidemiological data on baseline status and the multiple outcome measures are presented. Two research questions will be answered in chapter five and six using the data of this hysterectomy cohort. Research question 4:

What are the somatic and psychosocial risk and protective factors for pain, depression, physical functioning, global surgical recovery, and well-being after 3 and 12 months, in patients undergoing elective hysterectomy?

Research question 5:

What is the prevalence/incidence of chronic pain and depression, and what is the level of physical functioning, global surgical recovery, and well-being after 3 and 12 months, in patients undergoing elective hysterectomy? 


\section{REFERENCES}

1. The World Health Organization Quality of Life assessment (WHOQOL): position paper from the World Health Organization. Soc Sci Med. 1995; 41(10): 1403-1409.

2. The World Health Organization Quality of Life Assessment (WHOQOL): development and general psychometric properties. Soc Sci Med. 1998; 46(12): 1569-1585.

3. Allvin R, Berg K, Idvall E, et al. Postoperative recovery: a concept analysis. J Adv Nurs. 2007; 57(5): 552558.

4. Kleinbeck SV Self-reported at-home postoperative recovery. Res Nurs Health. 2000; 23(6): 461-472.

5. Lee L, Tran T, Mayo NE, et al. What does it really mean to "recover" from an operation? Surgery. 2014; 155(2): 211-216.

6. Turk DC, Dworkin RH, Allen RR, et al. Core outcome domains for chronic pain clinical trials: IMMPACT recommendations. Pain. 2003; 106(3): 337-345.

7. VanDenKerkhof EG, Peters ML, Bruce J Chronic pain after surgery: time for standardization? A framework to establish core risk factor and outcome domains for epidemiological studies. Clin J Pain. 2013; 29(1): 2-8.

8. Davies HTO, Crombie IK, Macrae WA, et al. Pain clinic patients in northern Britain. 1992.

9. Goren A, Mould-Quevedo J, daCosta DiBonaventura M Prevalence of pain reporting and associated health outcomes across emerging markets and developed countries. Pain Med. 2014; 15(11): 18801891.

10. Langley P, Muller-Schwefe G, Nicolaou A, et al. The societal impact of pain in the European Union: health-related quality of life and healthcare resource utilization. J Med Econ. 2010; 13(3): 571-581.

11. Langley P, Perez Hernandez C, Margarit Ferri C, et al. Pain, health related quality of life and healthcare resource utilization in Spain. J Med Econ. 2011; 14(5): 628-638.

12. Langley PC The societal burden of pain in Germany: health-related quality-of-life, health status and direct medical costs. J Med Econ. 2012; 15(6): 1201-1215.

13. Takura T, Ushida T, Kanchiku T, et al. The societal burden of chronic pain in Japan: an internet survey. J Orthop Sci. 2015; 20(4): 750-760.

14. Macrae WA Chronic pain after surgery. Br J Anaesth. 2001; 87(1): 88-98.

15. Werner MU, Kongsgaard UE I. Defining persistent post-surgical pain: is an update required? Br J Anaesth. 2014; 113(1): 1-4.

16. Hoofwijk DM, Fiddelers AA, Peters ML, et al. Prevalence and Predictive Factors of Chronic Postsurgical Pain and Poor Global Recovery One Year after Outpatient Surgery. Clin J Pain. 2015.

17. Kalliomaki ML, Sandblom G, Gunnarsson U, et al. Persistent pain after groin hernia surgery: a qualitative analysis of pain and its consequences for quality of life. Acta Anaesthesiol Scand. 2009; 53(2): 236-246.

18. Brandsborg B, Nikolajsen L, Kehlet $\mathrm{H}$, et al. Chronic pain after hysterectomy. Acta Anaesthesiol Scand. 2008; 52(3): 327-331.

19. Gartner R, Jensen MB, Nielsen J, et al. Prevalence of and factors associated with persistent pain following breast cancer surgery. JAMA. 2009; 302(18): 1985-1992.

20. Bayman EO,Brennan TJ Incidence and severity of chronic pain at 3 and 6 months after thoracotomy: meta-analysis. J Pain. 2014; 15(9): 887-897.

21. Grosu I, de Kock M New concepts in acute pain management: strategies to prevent chronic postsurgical pain, opioid-induced hyperalgesia, and outcome measures. Anesthesiol Clin. 2011; 29(2): 311-327.

22. Katz J, Seltzer Z Transition from acute to chronic postsurgical pain: risk factors and protective factors. Expert Rev Neurother. 2009; 9(5): 723-744.

23. Deumens $R$, Steyaert A, Forget $P$, et al. Prevention of chronic postoperative pain: cellular, molecular, and clinical insights for mechanism-based treatment approaches. Prog Neurobiol. 2013; 104: 1-37.

24. Gebhart GF Descending modulation of pain. Neurosci Biobehav Rev. 2004; 27(8): 729-737.

25. Goodin BR, McGuire L, Allshouse M, et al. Associations between catastrophizing and endogenous paininhibitory processes: sex differences. J Pain. 2009; 10(2): 180-190. 
26. Kehlet $\mathrm{H}$, Jensen TS, Woolf CJ Persistent postsurgical pain: risk factors and prevention. Lancet. 2006; 367(9522): 1618-1625.

27. Woolf CJ Central sensitization: implications for the diagnosis and treatment of pain. Pain. 2011; 152(3 Suppl): S2-15.

28. Treede RD, Jensen TS, Campbell JN, et al. Neuropathic pain: redefinition and a grading system for clinical and research purposes. Neurology. 2008; 70(18): 1630-1635.

29. Haroutiunian S, Nikolajsen L, Finnerup NB, et al. The neuropathic component in persistent postsurgical pain: a systematic literature review. Pain. 2013; 154(1): 95-102.

30. Loos MJ, Scheltinga MR, Mulders LG, et al. The Pfannenstiel incision as a source of chronic pain. Obstet Gynecol. 2008; 111(4): 839-846.

31. Shin JH, Howard FM Abdominal wall nerve injury during laparoscopic gynecologic surgery: incidence, risk factors, and treatment outcomes. J Minim Invasive Gynecol. 2012; 19(4): 448-453.

32. Devor M, De Koninck Y, Sommer C, Underlying Mechanisms of Neuropathic Pain, in 15th World Congress on Pain, S.N. Raja and C.L. Sommer, Editors. 2014, IASP Press: Buenos Aires, Argentina. p. 187-198.

33. Bauman AE Updating the evidence that physical activity is good for health: an epidemiological review 2000-2003. J Sci Med Sport. 2004; 7(1 Suppl): 6-19.

34. Bize R, Johnson JA, Plotnikoff RC Physical activity level and health-related quality of life in the general adult population: a systematic review. Prev Med. 2007; 45(6): 401-415.

35. Milton K, Macniven R, Bauman A Review of the epidemiological evidence for physical activity and health from low- and middle-income countries. Glob Public Health. 2014; 9(4): 369-381.

36. Rebar AL, Stanton R, Geard D, et al. A meta-meta-analysis of the effect of physical activity on depression and anxiety in non-clinical adult populations. Health Psychol Rev. 2015: 1-13.

37. Vogel T, Brechat PH, Lepretre PM, et al. Health benefits of physical activity in older patients: a review. Int J Clin Pract. 2009; 63(2): 303-320.

38. Ditmyer MM, Topp R, Pifer M Prehabilitation in preparation for orthopaedic surgery. Orthop Nurs. 2002; 21(5): 43-51; quiz 52-44.

39. Henschke N, Diong J Exercise reduces pain and improves physical function for people awaiting hip replacement surgery. Br J Sports Med. 2014; 48(6): 477-478.

40. Hulzebos EH, Smit Y, Helders PP, et al. Preoperative physical therapy for elective cardiac surgery patients. Cochrane Database Syst Rev. 2012; 11: CD010118.

41. Santa Mina D, Clarke $H$, Ritvo $P$, et al. Effect of total-body prehabilitation on postoperative outcomes: a systematic review and meta-analysis. Physiotherapy. 2014; 100(3): 196-207.

42. Handoll HH, Sherrington C, Mak JC Interventions for improving mobility after hip fracture surgery in adults. Cochrane Database Syst Rev. 2011(3): CD001704.

43. Hoogeboom TJ, Dronkers JJ, Hulzebos EH, et al. Merits of exercise therapy before and after major surgery. Curr Opin Anaesthesiol. 2014; 27(2): 161-166.

44. Baglioni C, Battagliese G, Feige B, et al. Insomnia as a predictor of depression: a meta-analytic evaluation of longitudinal epidemiological studies. J Affect Disord. 2011; 135(1-3): 10-19.

45. Maes M Depression is an inflammatory disease, but cell-mediated immune activation is the key component of depression. Progress in Neuro-Psychopharmacology \& Biological Psychiatry. 2011; 35(3): 664675.

46. McKenzie LH, Simpson J, Stewart M A systematic review of pre-operative predictors of post-operative depression and anxiety in individuals who have undergone coronary artery bypass graft surgery. Psychol Health Med. 2010; 15(1): 74-93.

47. Saboonchi F, Petersson LM, Wennman-Larsen A, et al. Changes in caseness of anxiety and depression in breast cancer patients during the first year following surgery: patterns of transiency and severity of the distress response. Eur J Oncol Nurs. 2014; 18(6): 598-604.

48. Smoller JW The Genetics of Stress-Related Disorders: PTSD, Depression, and Anxiety Disorders. Neuropsychopharmacology. 2015.

49. Wrench JM, Rayner G, Wilson SJ Profiling the evolution of depression after epilepsy surgery. Epilepsia. 2011; 52(5): 900-908. 


\section{Chapter 1}

50. Zieger M, Schwarz R, Konig HH, et al. Depression and anxiety in patients undergoing herniated disc surgery: relevant but underresearched - a systematic review. Cent Eur Neurosurg. 2010; 71(1): 26-34.

51. Ghoneim MM, O'Hara MW Depression and postoperative complications: an overview. BMC Surg. 2016; 16(1): 5 .

52. "well-being, n.", in Oxford English Dictionary, Oxford University Press.

53. Ryan RM, Deci EL On happiness and human potentials: a review of research on hedonic and eudaimonic well-being. Annu Rev Psychol. 2001; 52: 141-166.

54. Franz CE, Panizzon MS, Eaves LJ, et al. Genetic and environmental multidimensionality of well- and illbeing in middle aged twin men. Behav Genet. 2012; 42(4): 579-591.

55. Diener E, Suh EM, Lucas RE, et al. Subjective well-being: Three decades of progress. Psychological Bulletin. 1999; 125(2): 276-302.

56. Tsimopoulou I, Pasquali S, Howard R, et al. Psychological Prehabilitation Before Cancer Surgery: A Systematic Review. Ann Surg Oncol. 2015; 22(13): 4117-4123.

57. Nelson EA, Dowsey MM, Knowles SR, et al. Systematic review of the efficacy of pre-surgical mind-body based therapies on post-operative outcome measures. Complement Ther Med. 2013; 21(6): 697-711.

58. Arthur HM, Daniels C, McKelvie R, et al. Effect of a preoperative intervention on preoperative and postoperative outcomes in low-risk patients awaiting elective coronary artery bypass graft surgery. A randomized, controlled trial. Ann Intern Med. 2000; 133(4): 253-262.

59. Furze G, Dumville JC, Miles JN, et al. "Prehabilitation" prior to CABG surgery improves physical functioning and depression. Int J Cardiol. 2009; 132(1): 51-58.

60. Tefikow S, Barth J, Maichrowitz S, et al. Efficacy of hypnosis in adults undergoing surgery or medical procedures: a meta-analysis of randomized controlled trials. Clin Psychol Rev. 2013; 33(5): 623-636.

61. Parbrook GD, Steel DF, Dalrymple DG Factors predisposing to postoperative pain and pulmonary complications. A study of male patients undergoing elective gastric surgery. Br J Anaesth. 1973; 45(1): 21-33.

62. Cryer HM, Howard DA, Garrison RN Liver cirrhosis and biliary surgery: assessment of risk. South Med J. 1985; 78(2): 138-141.

63. Parkhouse J, Lambrechts W, Simpson BR The incidence of postoperative pain. Br J Anaesth. 1961; 33: 345-353.

64. Stovall TG, Ling FW, Crawford DA Hysterectomy for chronic pelvic pain of presumed uterine etiology. Obstet Gynecol. 1990; 75(4): 676-679.

65. Peters ML, Sommer M, de Rijke JM, et al. Somatic and psychologic predictors of long-term unfavorable outcome after surgical intervention. Ann Surg. 2007; 245(3): 487-494.

66. Hoofwijk DM, Fiddelers AA, Peters ML, et al. Prevalence and Predictive Factors of Chronic Postsurgical Pain and Poor Global Recovery 1 Year After Outpatient Surgery. Clin J Pain. 2015; 31(12): 1017-1025.

67. Janssen KJ, Kalkman CJ, Grobbee DE, et al. The risk of severe postoperative pain: modification and validation of a clinical prediction rule. Anesth Analg. 2008; 107(4): 1330-1339.

68. Kalkman CJ, Visser K, Moen J, et al. Preoperative prediction of severe postoperative pain. Pain. 2003; 105(3): 415-423.

69. Sipila R, Estlander AM, Tasmuth $\mathrm{T}$, et al. Development of a screening instrument for risk factors of persistent pain after breast cancer surgery. Br J Cancer. 2012; 107(9): 1459-1466.

70. Filbay SR, Ackerman IN, Russell TG, et al. Health-related quality of life after anterior cruciate ligament reconstruction: a systematic review. Am J Sports Med. 2014; 42(5): 1247-1255.

71. Wirken L, van Middendorp H, Hooghof CW, et al. The Course and Predictors of Health-Related Quality of Life in Living Kidney Donors: A Systematic Review and Meta-Analysis. Am J Transplant. 2015; 15(12): 3041-3054.

72. Statistic Netherlands (CBS). Operaties in het ziekenhuis; soort opname, leeftijd en geslacht, 1995-2010 2010 22-11-2014]; Available from: http://statline.cbs.nl/Statweb/publication/?DM=SLNL\&PA=80386NED \&D1=a\&D2=|\&D3=2-3\&D4=0,16-17\&D5=|\&HDR=T\&STB=G1,G4,G2,G3\&VW=T.

73. Sheldon KW The false fear of disc surgery. Nebr State Med J. 1949; 34(9): 326-329.

74. Walsh JJ, Psychotherapy. 1912: D Appleton \& Company. 
75. Ogden RM Music and Medicine, by Dorothy M. Schullian and Max Schoen (Book Review). The American Journal of Psychology. 1948; 61(4): 595-598.

76. Hinrichs-Rocker A, Schulz K, Jarvinen I, et al. Psychosocial predictors and correlates for chronic postsurgical pain (CPSP) - a systematic review. Eur J Pain. 2009; 13(7): 719-730.

77. Munafo MR, Stevenson J Anxiety and surgical recovery. Reinterpreting the literature. J Psychosom Res. 2001; 51(4): 589-596.

78. Rosenberger PH, Jokl P, Ickovics J Psychosocial factors and surgical outcomes: an evidence-based literature review. J Am Acad Orthop Surg. 2006; 14(7): 397-405. 



\section{Chapter}

\section{Preoperative anxiety and catastrophizing: a systematic review and meta-analysis of the association with chronic postsurgical pain}

This chapter is published as: Maurice Theunissen, Madelon L. Peters, Julie Bruce, HansFritz Gramke, and Marco A. Marcus (2012). Preoperative anxiety and catastrophizing: a systematic review and meta-analysis of the association with chronic postsurgical pain. Clinical Journal of Pain, 28, 819-841. 


\section{ABSTRACT}

Objectives: Anxiety and pain catastrophizing predict acute postoperative pain. However, it is not well established whether they also predict chronic postsurgical pain (CPSP). The aim of this systematic review and meta-analysis was to investigate whether high levels of preoperative anxiety or pain catastrophizing are associated with an increased risk of CPSP.

Methods: Electronic search databases included PubMed and PsychINFO. Additional literature was obtained by reference tracking and expert consultation. Studies from 1958 until October 2010, investigating the association between preoperative anxiety or pain catastrophizing and CPSP in adult surgery patients, were assessed. The primary outcome was the presence of pain at least 3 months postoperatively.

Results: Twenty-nine studies were included; 14 instruments were used to assess anxiety or pain catastrophizing. Sixteen studies (55\%) reported a statistically significant association between anxiety or pain catastrophizing and CPSP. The proportion of studies reporting a statistically significant association was $67 \%$ for studies of musculoskeletal surgery and $36 \%$ for other types of surgery. There was no association with study quality, but larger studies were more likely to report a statistically significant relationship. The overall pooled odds ratio, on the basis of 15 studies, ranged from 1.55 (95\% confidence interval, $1.10-2.20)$ to 2.10 (95\% confidence interval, 1.49-2.95). Pain catastrophizing might be of higher predictive utility compared with general anxiety or more specific pain-related anxiety.

Discussion: There is evidence that anxiety and catastrophizing play a role in the development of CPSP. We recommend that anxiety measures should be incorporated in future studies investigating the prediction and transition from acute to chronic postoperative pain. 


\section{BACKGROUND}

Postoperative pain is of major concern after surgery because of the impact on postoperative recovery, quality of life, and the risk of acute postoperative pain (APP) in the first postoperative days progressing to become chronic postsurgical pain (CPSP). ${ }^{1}$ Many studies report unacceptably high levels of APP after surgery. ${ }^{2-5}$ Predictive factors for APP include factors such as younger age and prior pain experience, in addition to psychological factors. ${ }^{2,6,7}$ In particular, the influence of preoperative anxiety on APP is well established. ${ }^{6,8-10}$ Both state anxiety, a temporary condition experienced in a specific situation, and trait anxiety, a general tendency to perceive situations as threatening, can play a role in the experience of postoperative pain. ${ }^{11}$ Another psychological factor that has been found to be predictive of APP is pain catastrophizing. ${ }^{12-14}$ Pain catastrophizing, defined as the tendency to magnify the threat value of pain and to feel helpless in the context of pain, is highly correlated with both state and trait anxiety and with more specific pain-related fears. ${ }^{15-17}$

The question we seek to answer in this systematic review is whether preoperative anxiety and catastrophizing also predict chronic pain after surgery. Since the early 1990s, there has been a growth in clinical and epidemiological research investigating the prevalence, predictors, and burden of CPSP: the potential long-term impact on quality of life and the societal and economic burden from CPSP has been well reported. ${ }^{18-20}$ Chronic pain is defined by the IASP as pain persisting beyond the normal time of healing, generally after 3 months. ${ }^{21}$ More specifically, for CPSP, Macrae refers to pain that develops after a surgical procedure; is present for at least 2 months; no other cause for the pain can be identified; and is not the result of a continuing, preexisting problem. $^{22}$ Prevalence estimates for CPSP have ranged from $10 \%$ to $50 \%$ depending on the specific study, methodology, timing of follow-up, and type of surgical procedure. ${ }^{18,23}$ The role of psychological factors in the development of CPSP has been suggested ${ }^{23,24}$ but is not as well established as in the case of APP.

One of the most recent systematic reviews targeting the role of psychosocial predictors of CPSP in patients undergoing various types of surgery was performed by HinrichsRocker et al. $^{25}$ Depression, psychological vulnerability, and stress were identified as psychological factors showing a likely correlation to CPSP. For many other psychological factors, including anxiety, the results were inconclusive. However, since the publication of this review, which included papers published up to 2006, several new studies have examined preoperative anxiety and/or pain catastrophizing in relation to CPSP. To our knowledge, no formal meta-analysis has been conducted using data from multiple primary studies. Therefore, it is timely to conduct an updated review and meta-analysis that specifically focuses on the predictive value of anxiety and pain catastrophizing for CPSP after different types of surgeries.

For the purpose of this review, we consider all studies that have included either a measure of general (state or trait) anxiety, specific pain-related anxiety, or pain 
catastrophizing. According to the fear-avoidance model of chronic pain, pain catastrophizing elicits pain-related anxiety, and both are considered major determinants of pain persistence after an acute injury. ${ }^{26}$ In addition, a hierarchical model of general and specific anxieties has been proposed in which both pain catastrophizing and painrelated anxiety are seen as pain-specific constructs that are subsumed under the higher order construct of trait anxiety. ${ }^{27}$ A high global anxiety disposition is hypothesized to lead to pain-related anxieties in acute pain situations, for example during surgery. Because it may also be speculated that more proximal constructs (ie, pain-specific anxieties) have higher predictive value than more distal constructs (ie, global trait or state anxiety), in addition to pooling the results of all classes of predictors together, we will also examine the role of global anxiety, pain-specific anxiety, and pain catastrophizing separately.

An additional question is whether an association between anxiety and pain catastrophizing would be more evident in patients undergoing surgery involving the musculoskeletal system, that is lumbar, hip, knee, and shoulder surgery. The fearavoidance model of chronic pain was originally developed to explain the persistence of nonspecific musculoskeletal pain. A key role is assigned to avoidance of physical activity: fear-induced avoidance results in decreased physical fitness, which in turn fuels the cycle of increased disability and pain. ${ }^{26}$ Persistent avoidance might therefore be especially detrimental in the recovery from musculoskeletal surgery. Moreover, musculoskeletal surgery patients may be more likely to have chronic or ongoing preoperative pain that could be the primary indication for surgery, and preexisting pain is in itself a determinant of CPSP. ${ }^{24,28}$

Two systematic reviews have specifically focused on psychological predictors of outcome after lumbar surgery. Den Boer et al. ${ }^{29}$ found evidence that, in addition to somatization and passive avoidance coping strategies, preoperative anxiety may predict unfavorable outcomes (including pain) after lumbar surgery. More recently, Celestin et al. ${ }^{30}$ performed a systematic review of psychosocial variables as predictors of outcomes (including pain) after lumbar surgery and spinal cord stimulation. The results suggest a possible association between preoperative levels of anxiety, depression, coping, somatization, hypochondriasis, and poor outcome (including pain) after lumbar procedures. Thus, the evidence that preoperative anxiety and pain catastrophizing are predictive of persistent pain in patients undergoing lumbar surgery seems to be more consistent than for other types of surgery. Whether this also holds for other surgeries involving the musculoskeletal system remains to be determined.

The aim of this systematic review was to summarize current evidence concerning the hypothesis that high levels of preoperative anxiety or pain catastrophizing are associated with an increased risk of CPSP. The outcome in this review will be limited to intensity and/or presence of pain or a composite score including pain presence/intensity at 3 months or longer after surgery. Furthermore, we will investigate whether the hy- 
pothesized association between anxiety and CPSP is consistent between studies of musculoskeletal surgery and the other types of surgery.

\section{MATERIALS AND METHODS}

\section{Search strategy and study selection}

Studies considered eligible for inclusion within the review were observational cohort studies, case-control studies, and RCTs of adult surgical patients undergoing all types of surgery except dental surgery. Studies published in English, German, French, and Dutch were eligible for inclusion. Only studies in which anxiety data were collected before surgery and pain data were collected after surgery were included.

Bibliographic searches were conducted on PubMed (National Center for Biotechnology Information, NCBI, Bethesda) and PsychINFO (EBSCO Publishing, Ipswich) databases for literature published between 1958 and October 2010. Search strategies were initially developed and piloted using MEDLINE but a higher return rate was obtained using PubMed. Final search strategies used a combination of Medical Subject Heading (MeSH) and text words: ((presurgical or preoperative) AND ("Fear"[Mesh] OR "Anxiety"[Mesh] OR "Anxiety Disorders"[Mesh], fear or anxiety or coping or catastrophizing or psychosocial)) AND ("Pain, Postoperative"[Mesh], pain or acute pain or chronic pain or persistent pain) AND (postoperative or postsurgical). Electronic searches were limited to studies of adults. Additional searches were carried out by reference tracking and expert consultation. The search was performed by M.P. (psychologist) and M.T. (epidemiologist). The abstracts retrieved from electronic and hand searching were assessed independently. Subsequently, all studies describing surgical patients not having dental surgery, with an assessment of preoperative psychosocial state and postoperative measurement of pain at least 3 months after surgery, were selected for full-text reading. If any aspect of the abstract was unclear, the full text was obtained and assessed. There was no use of conference abstracts. To enable meta-analysis, all authors of publications that did not include data suitable for the calculation of pooled odds ratios (OR) were contacted for additional data to contribute to the review.

\section{Outcomes and predictors}

The primary outcome was pain assessed at least 3 months after surgery. In addition, multidimensional outcome measures were included if they incorporated a pain component or measure. Predictors related to preoperative anxiety were eligible for inclusion if they assessed concepts of anxiety, surgical fear, or pain catastrophizing. If multidimensional instruments were used preoperatively or postoperatively, these had to include and report at least 1 relevant subscale. 


\section{Data extraction and critical appraisal}

Extracted data of the selected studies were tabulated, reporting author, publication date, country, study design, follow-up duration, type of surgery, sample size, outcome measure(s), predictor(s), effect, and type of statistics used. Study quality was assessed using an 8-item checklist (scores 0 to 8). The quality items were based on the Critical Appraisal Checklist for Cohort/Case Control appraisal of the Joanna Briggs institute ${ }^{31}$ and the checklist for measuring study quality developed by Downs and Black, ${ }^{32}$ which were adapted for use (Appendix). Unblinded quality assessment was performed independently by M.T. and V.T. (MSc in Medicine). Interrater agreement on the total score was assessed using the weighted quadratic $\mathrm{k}^{33}$; interrater agreement per item was assessed using the $\mathrm{k}$ statistic. In cases of divergent scoring, a consensus meeting with a third rater (M.L.P.) led to the final judgment. Chi-square tests were used for a sensitivity analysis on study quality with regard to the proportion of studies reporting statistically significant results, type of surgery (musculoskeletal surgery vs. remaining types of surgery), and publication date (before/after 2008). The publication of the STROBE statement, ${ }^{34}$ providing guidelines for the reporting of observational studies, was the reason for selecting 2008 as the cutoff for publication date as a proxy quality indicator. The weighted quadratic $\mathrm{k}$ was calculated using kappapls.exe software [Software developed by A.G. Kessels, MD, MSc, Department of Clinical Epidemiology and Medical Technology Assessment (KEMTA), MUMC+, Maastricht, The Netherlands]. The $\mathrm{k}$ statistic and $\chi^{2}$ tests were performed using the Statistical Package for the Social Sciences (SPSS version 18, Chicago, IL). Meta-analysis was performed using STATA version 11.2 (Stata Corp., College Station, TX).

Because of heterogeneity in the reported data, initial pooling of the results of all 29 included studies was restricted to vote counting and descriptive analysis. At the study level, the total number of studies reporting any statistically significant positive association between preoperative anxiety or catastrophizing and CPSP is reported. At the predictor level, the number of studies reporting any statistically significant positive association between a specific predictor and any of the pain-related outcomes, versus the total number of studies using that predictor, is reported.

Second, a random-effects meta-analysis was performed on a subset of 15 studies that provided data to allow the calculation of pooled OR. Published data (9 references) and unpublished data, which were provided at request by 6 authors, were used. If raw data were provided, patients were classified as having CPSP yes/no and preoperative anxiety or catastrophizing yes/no by a median split. Because of the nonparametric distribution, statistical pooling was performed using log-transformed ORs. Subsequently, the results were converted by taking the exponential of the log OR. Because of the heterogeneity in predictor and outcome measures, the random-effects model was chosen. Where a study presented more than one predictor/outcome combination, we divided 
the results into a maximum effect scenario (highest $O R$ ) and a minimum effect scenario (lowest OR) to avoid overrepresentation of studies reporting more than 1 outcome.

\section{RESULTS}

The process of study selection is presented in the flow chart (Figure 1). A total of 71 articles from 512 references were obtained and critically appraised; of these, 29 studies were considered eligible for inclusion in the review ( $N=6628$ patients). Musculoskeletal surgery was performed in 18 studies ( $n=4963$ patients) and 11 studies concerned other types of surgery $(n=1665)$. All studies were either performed in the United States (11) or western Europe (18). One study was a nested cohort study within an RCT; all other studies were observational cohort studies. Data on the selected studies are presented in Table 1. 


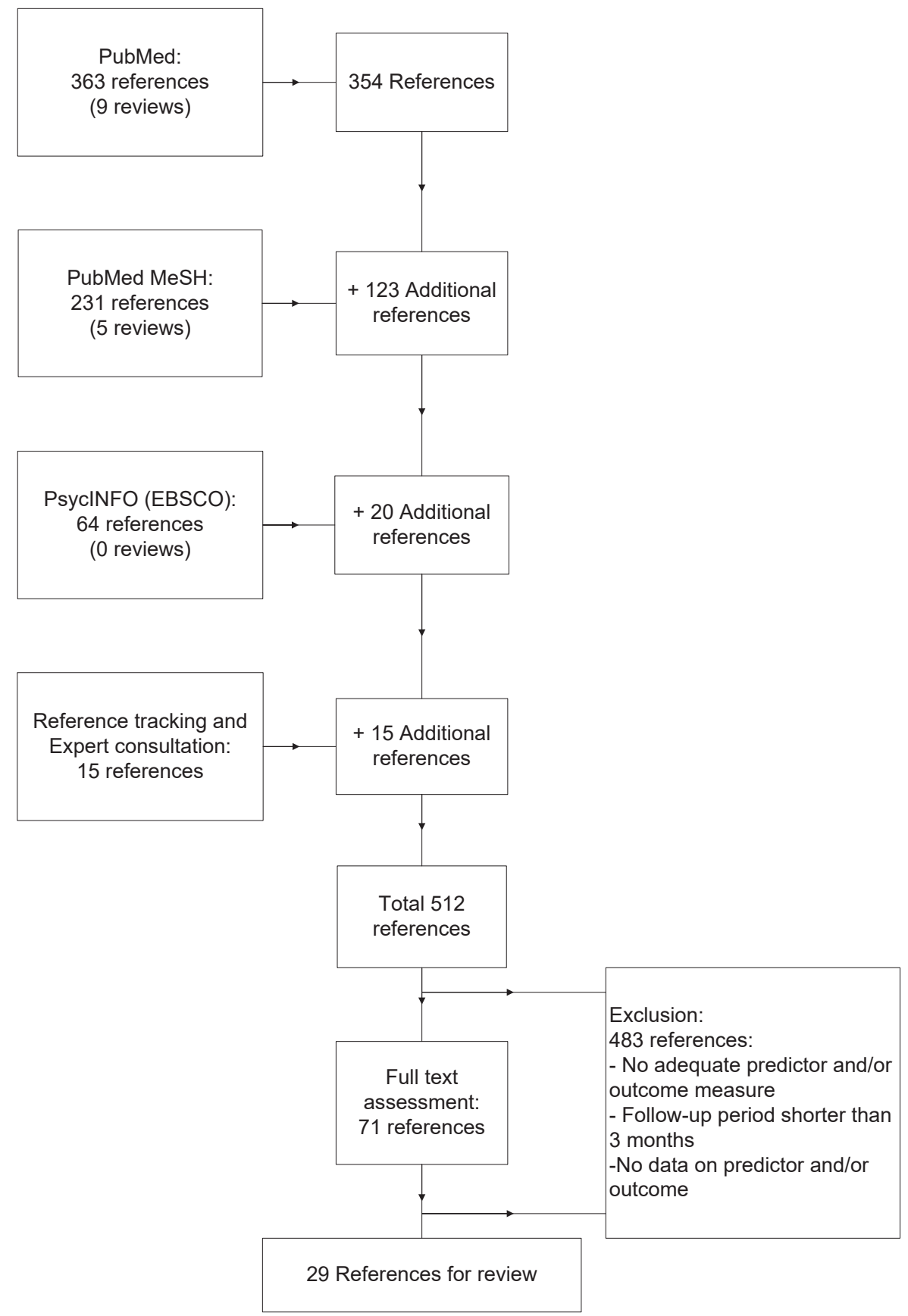

Figure 1. Flow chart study selection 
Preoperative anxiety and catastrophizing

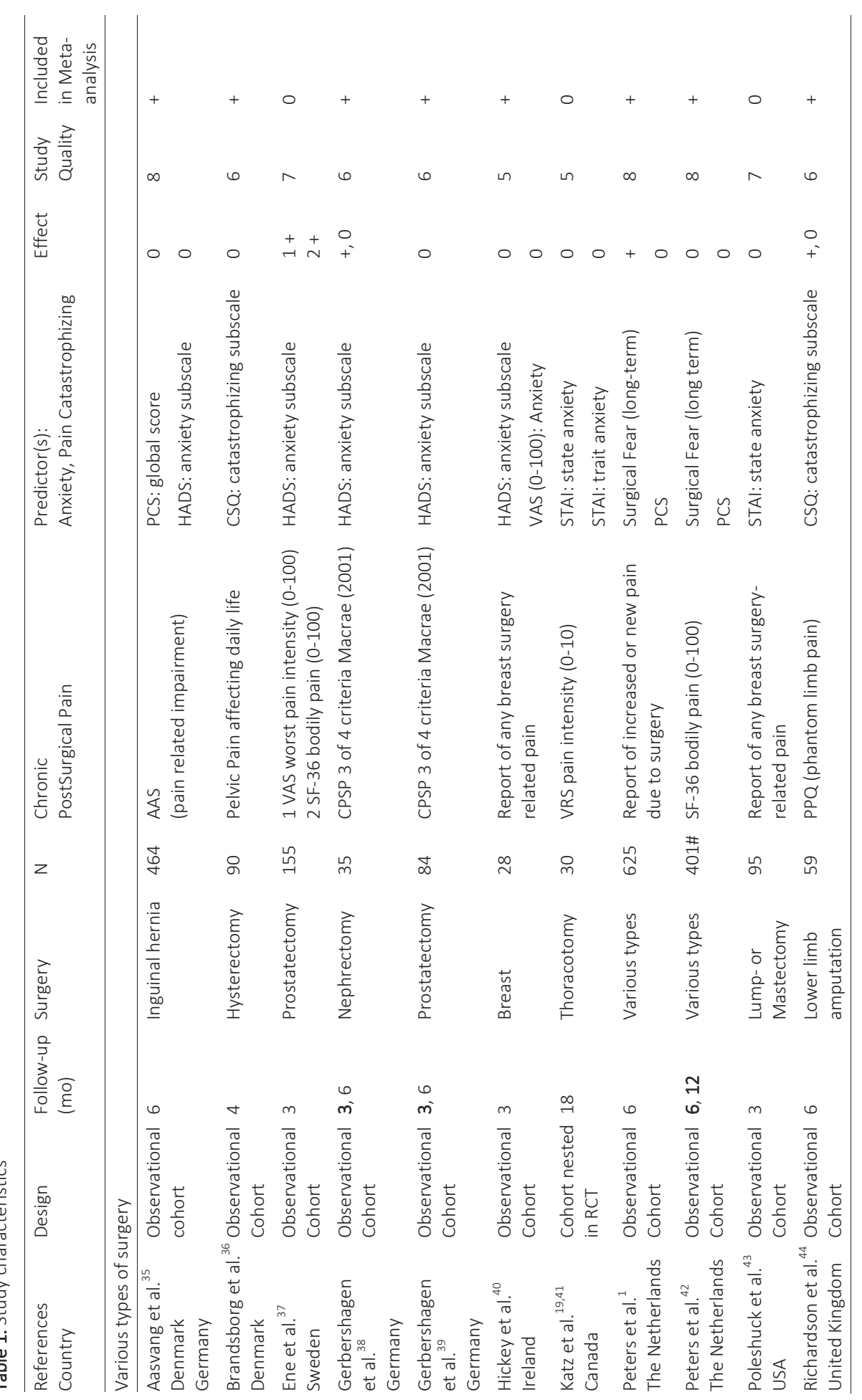




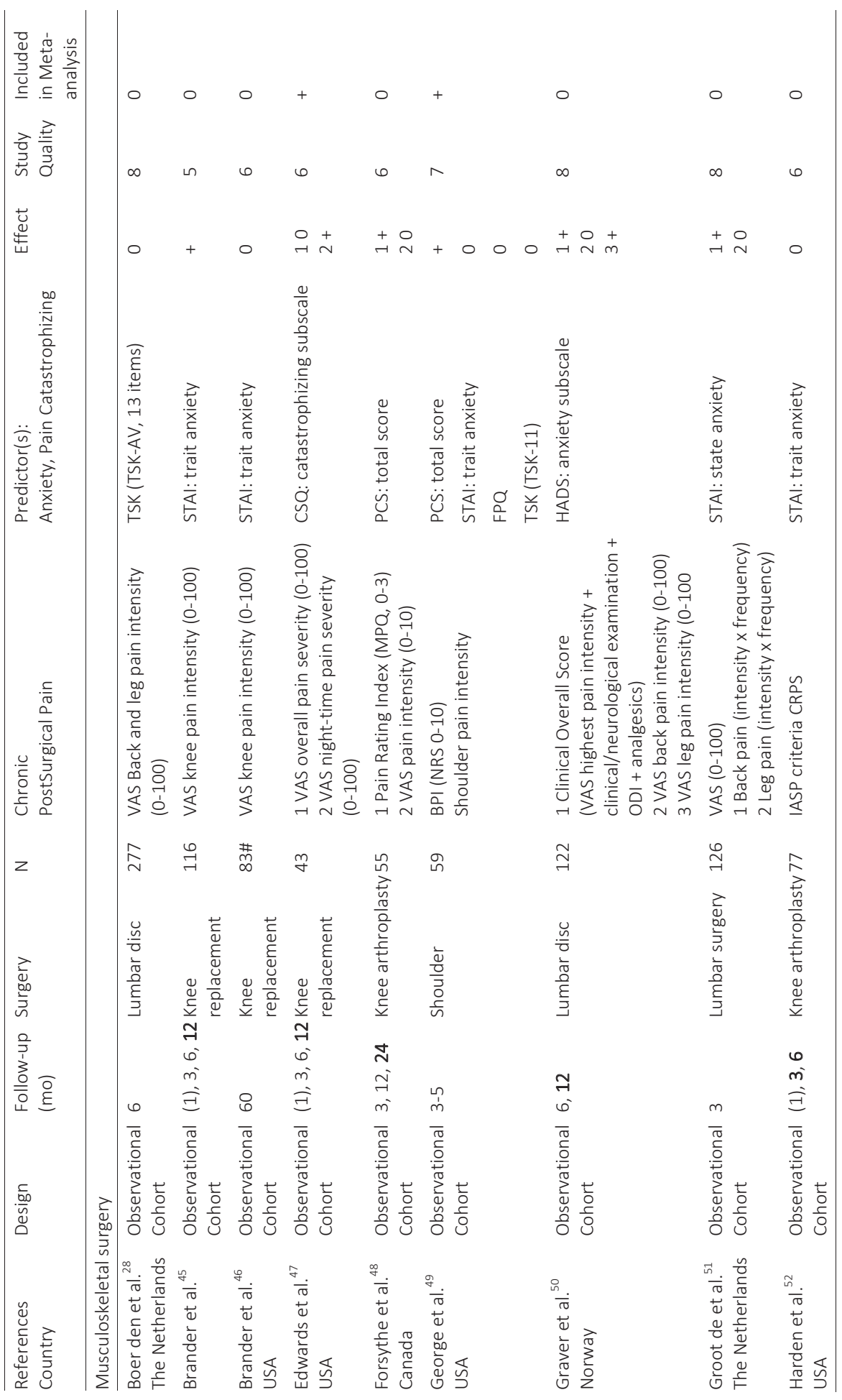


Preoperative anxiety and catastrophizing

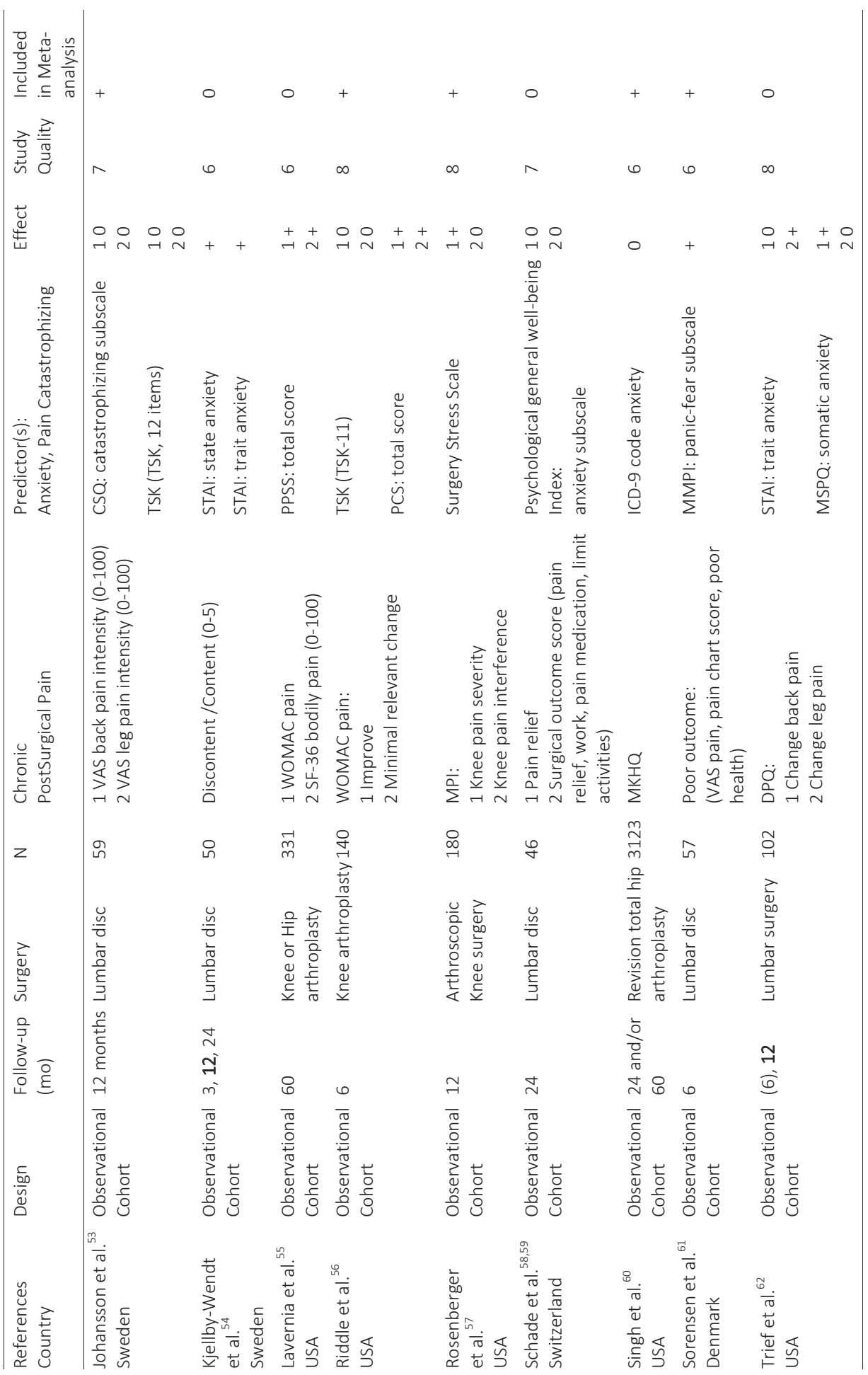


Chapter 2

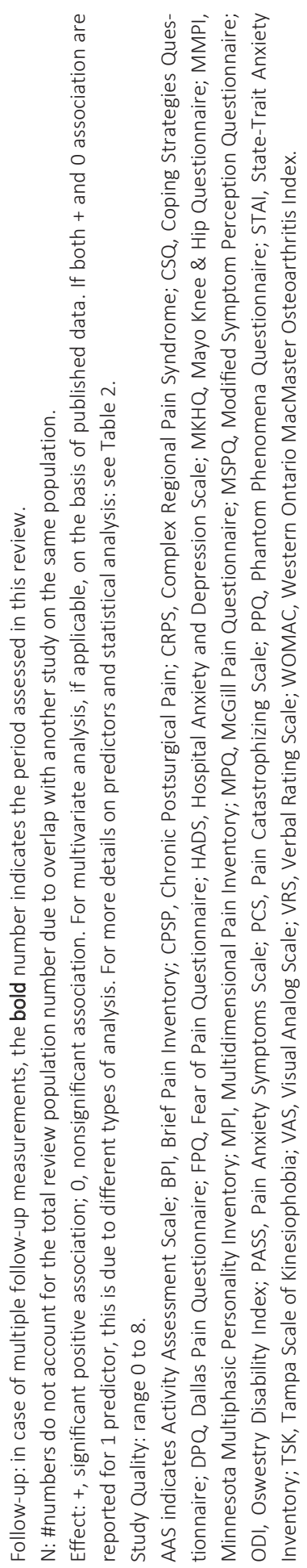




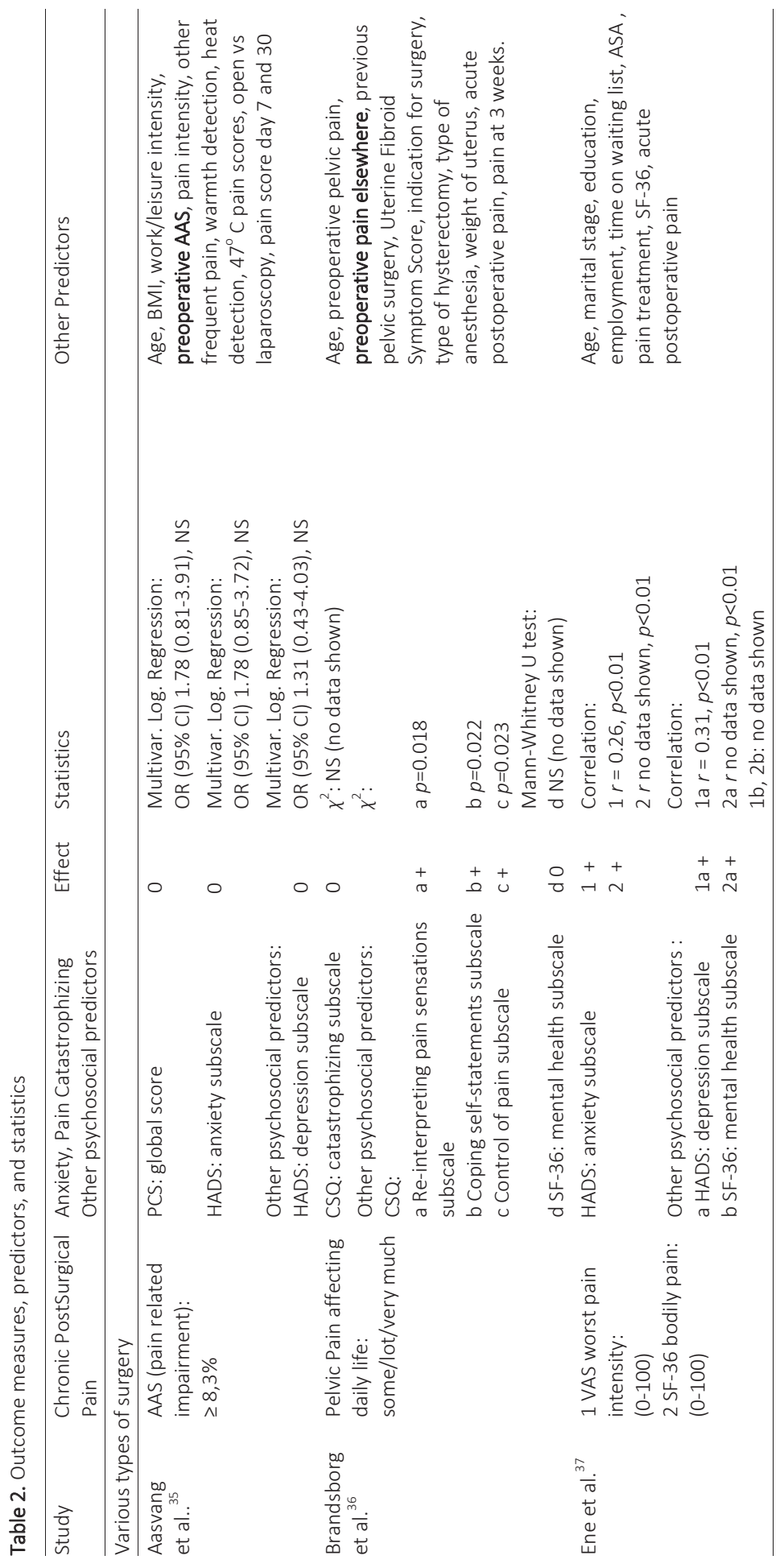




\section{Chapter 2}

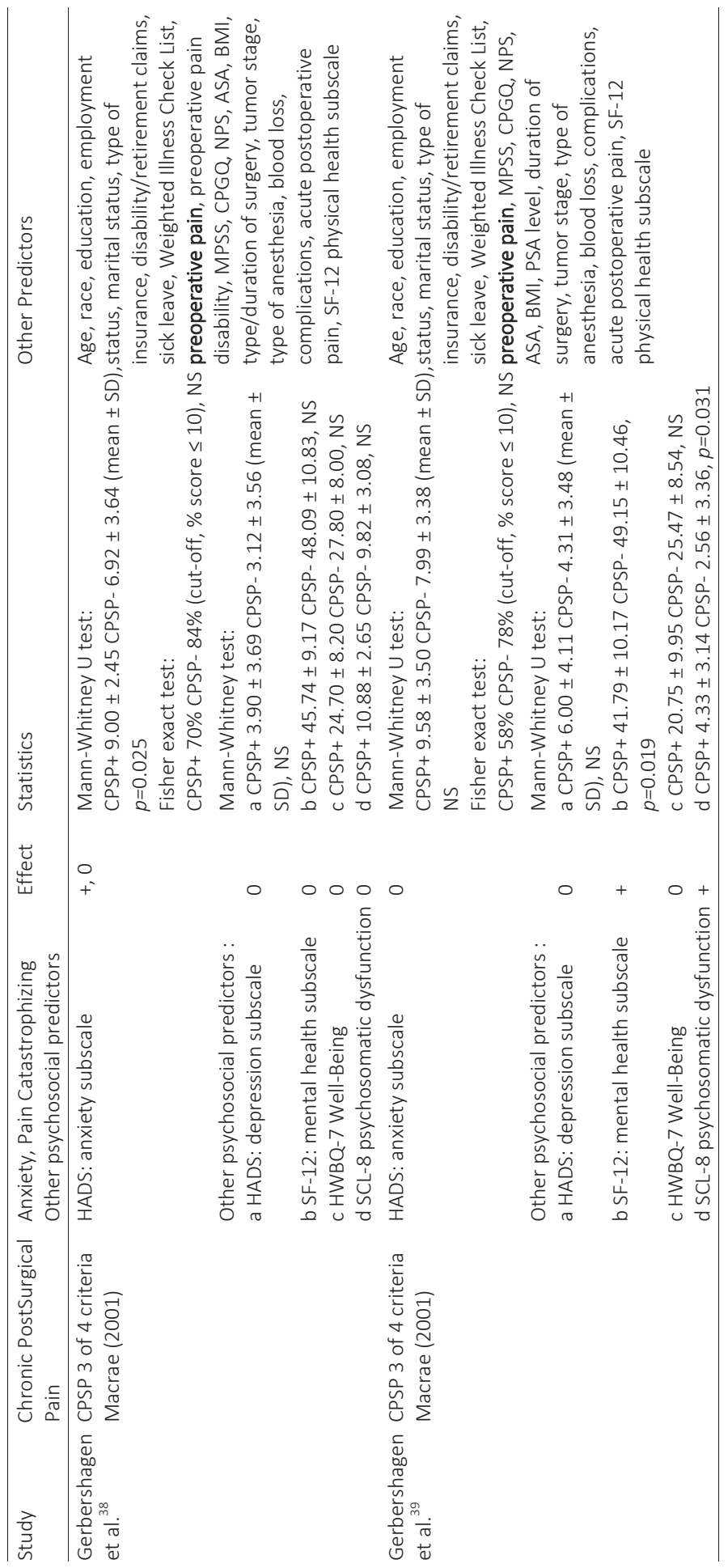




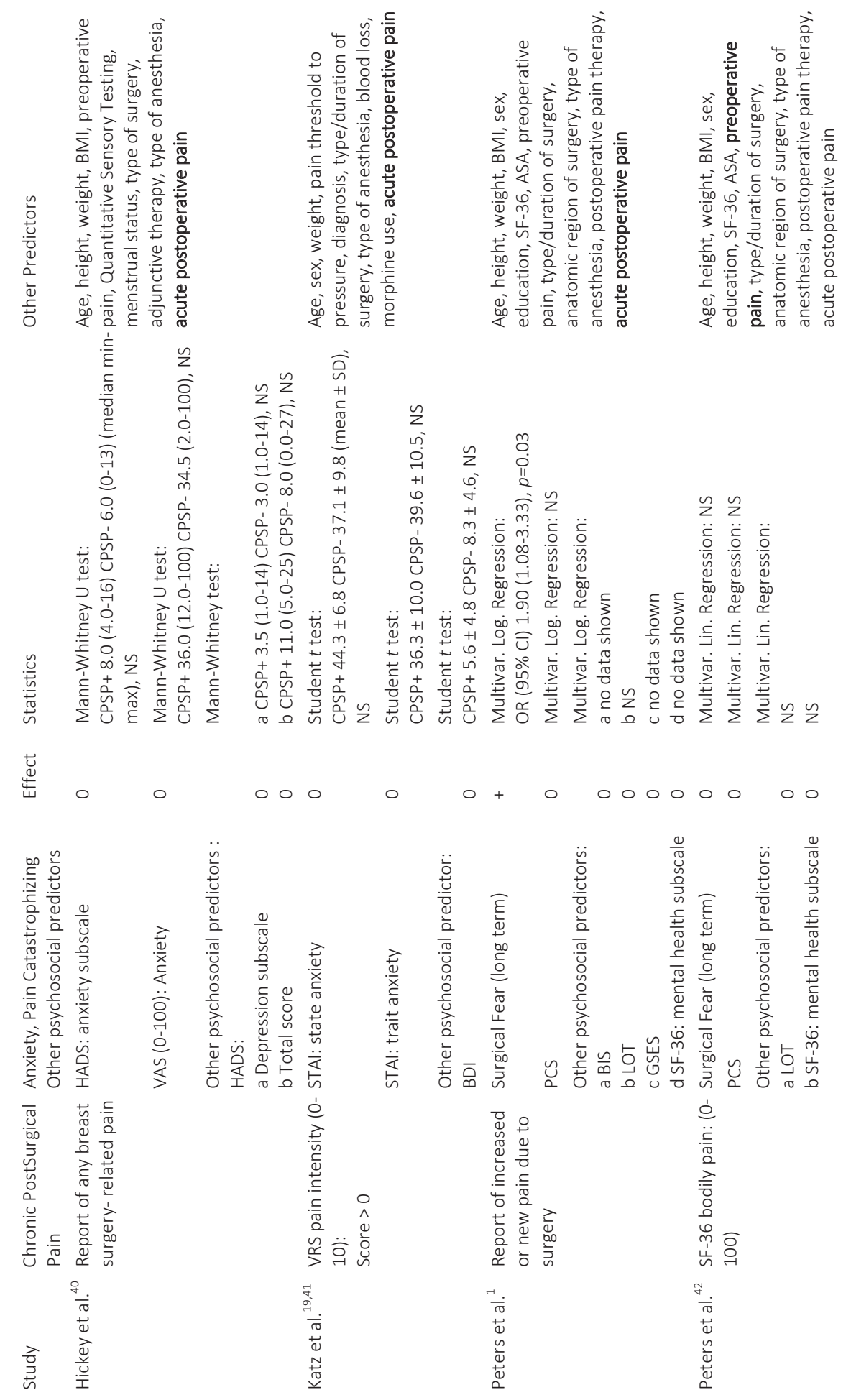




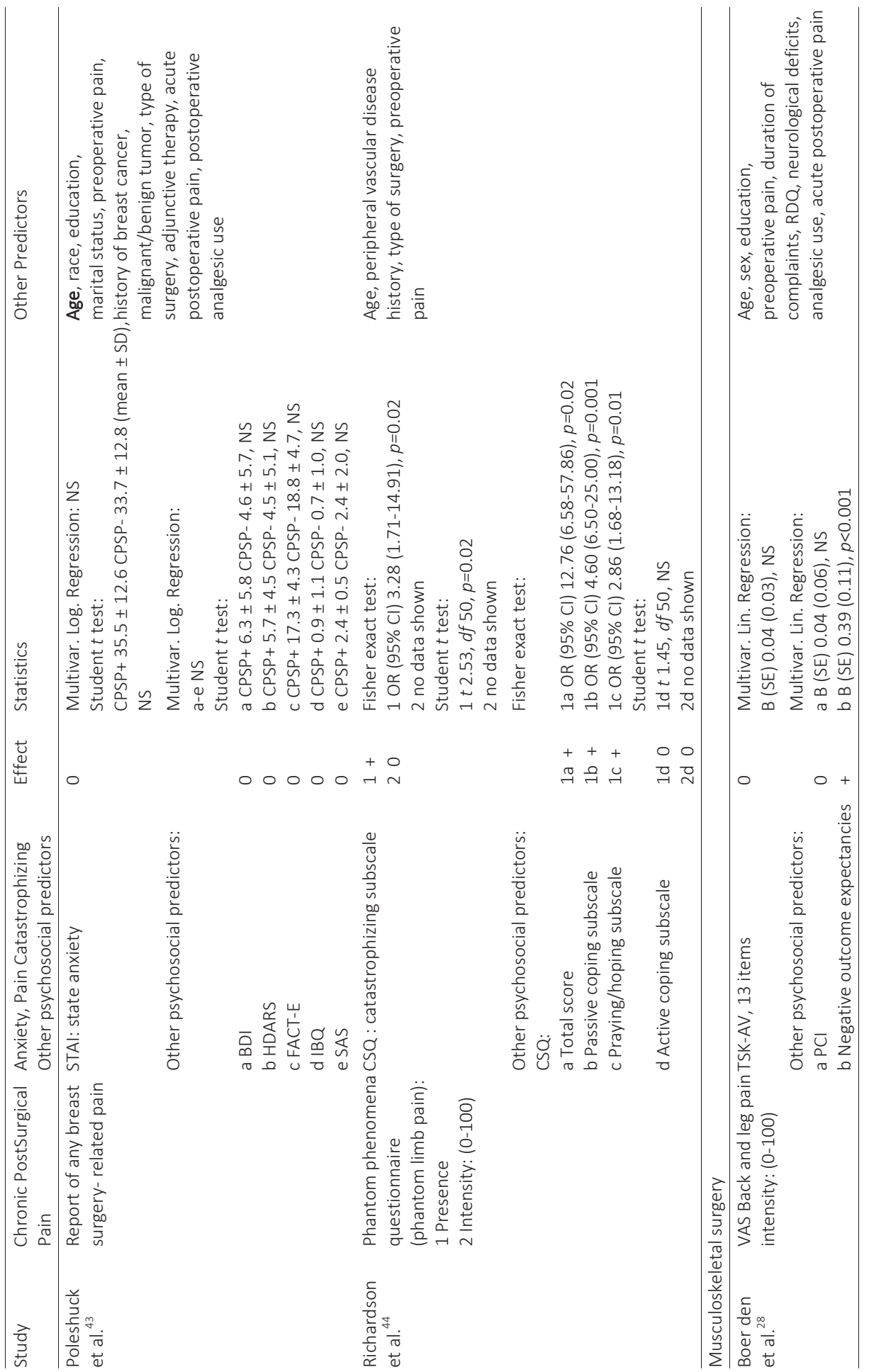




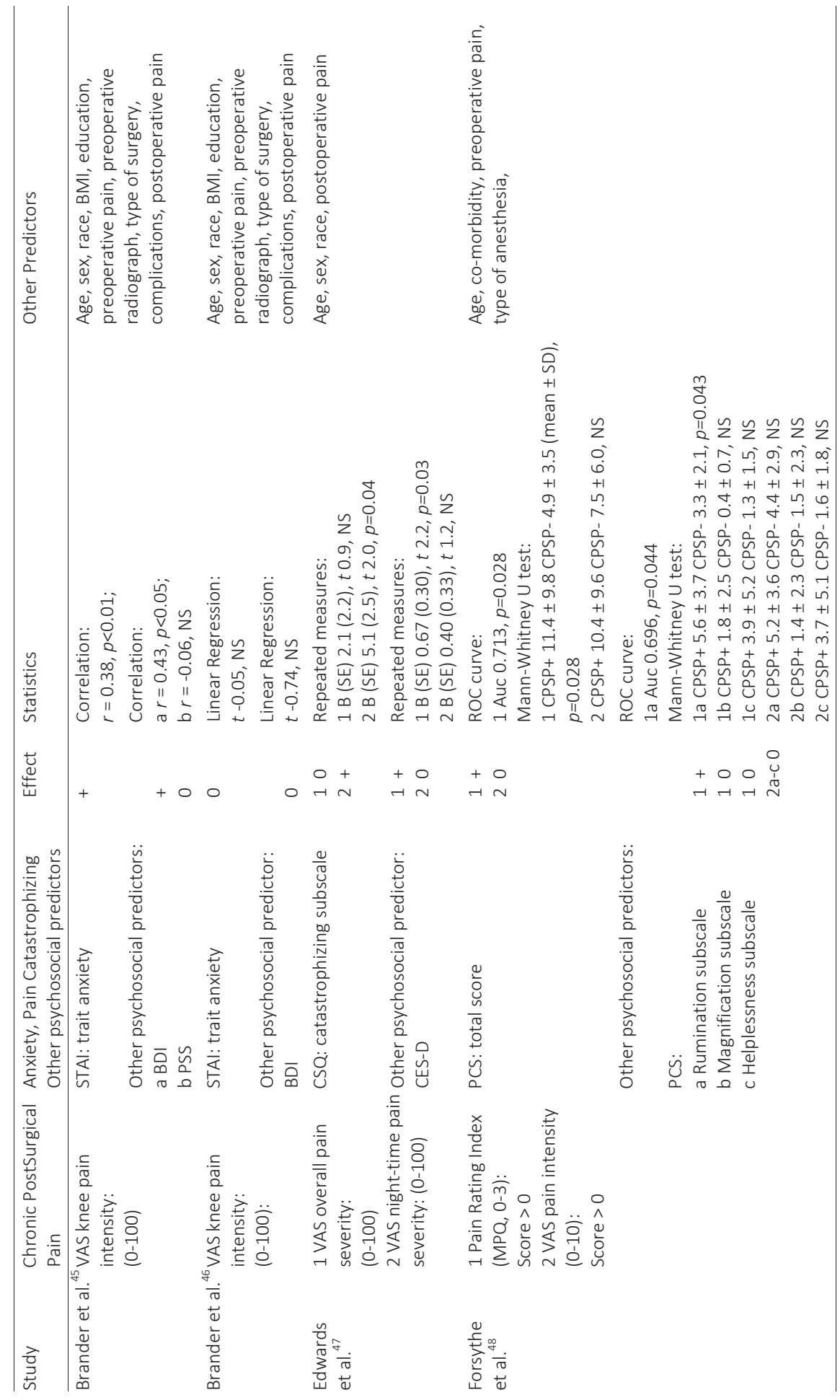




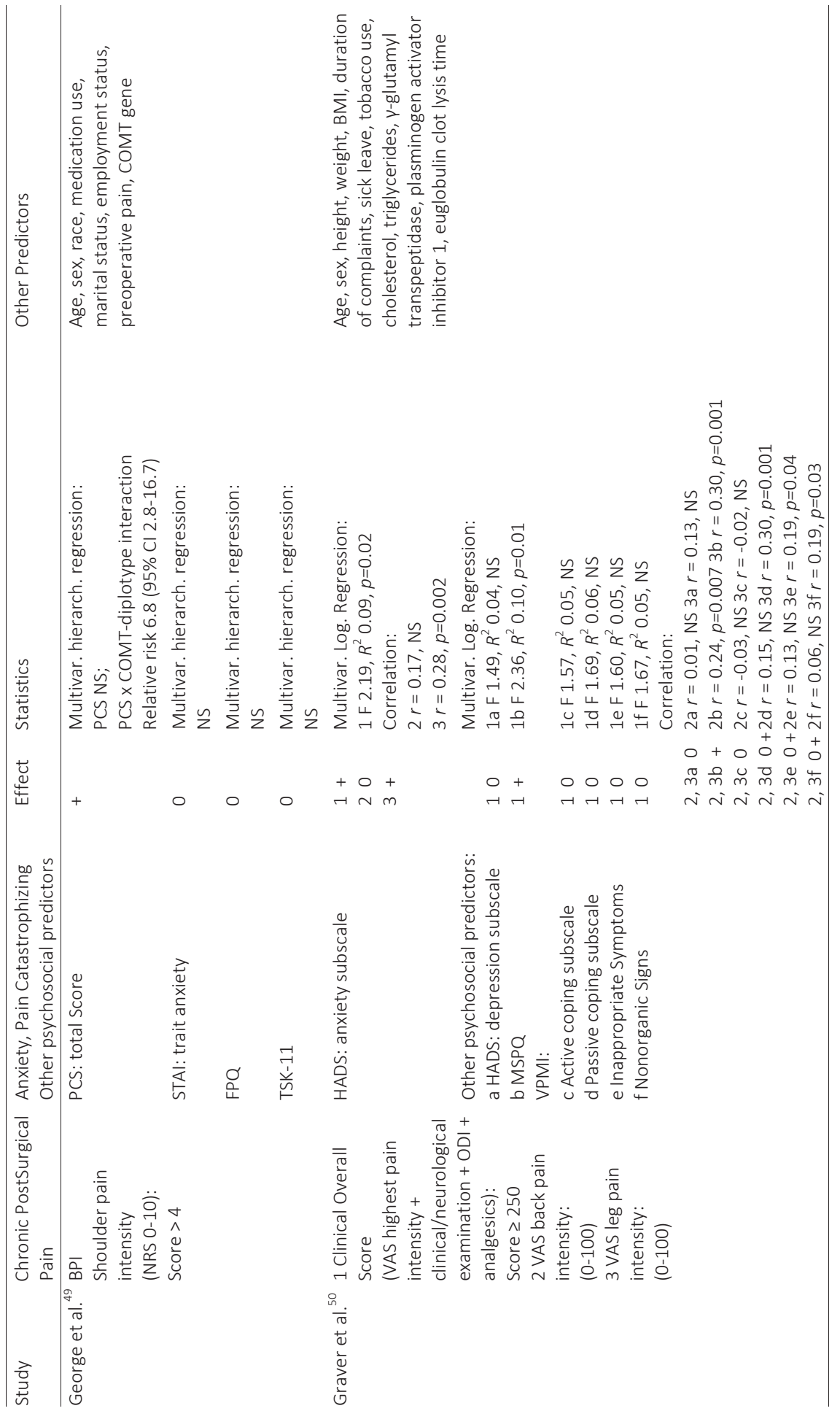




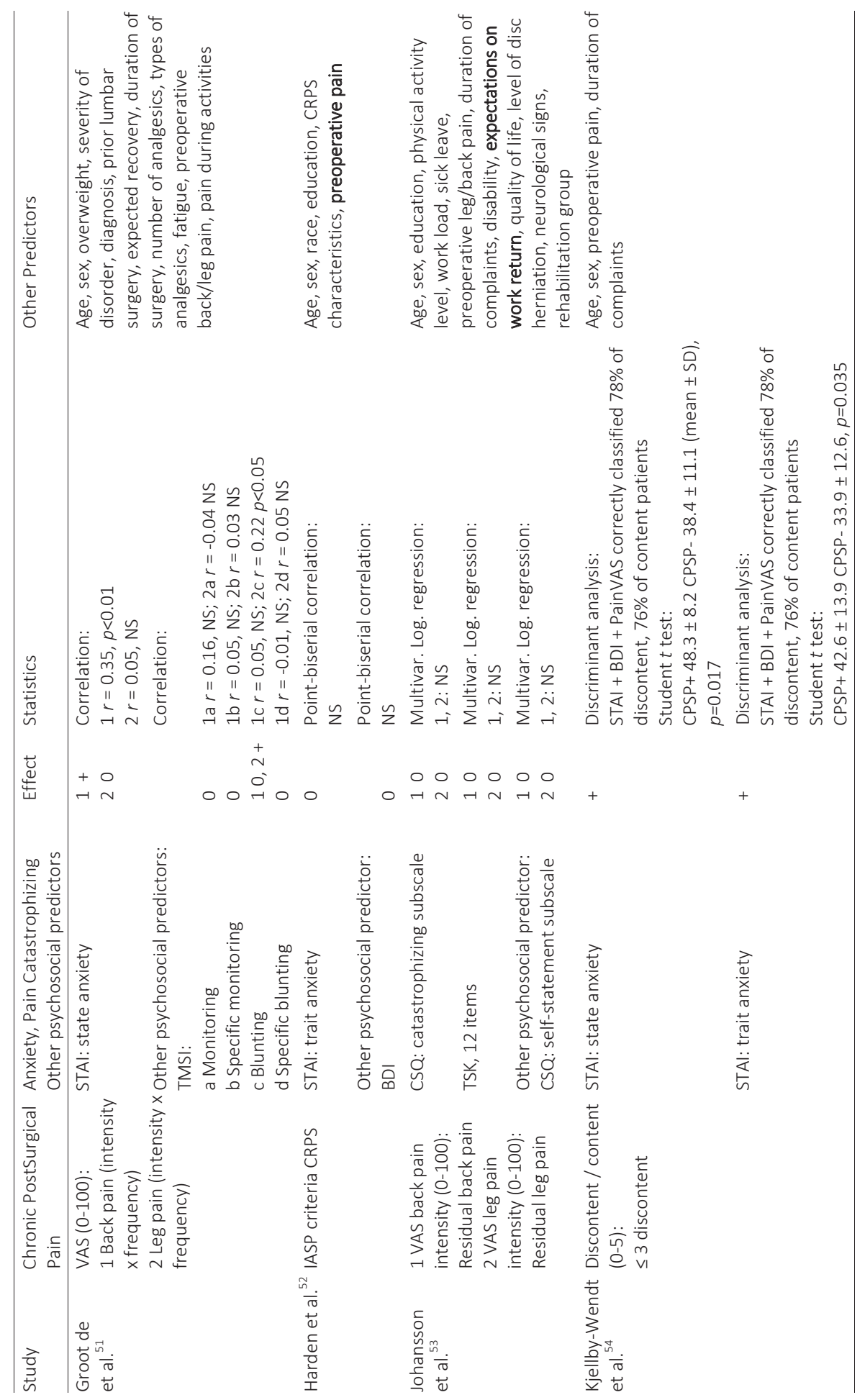


Chapter 2

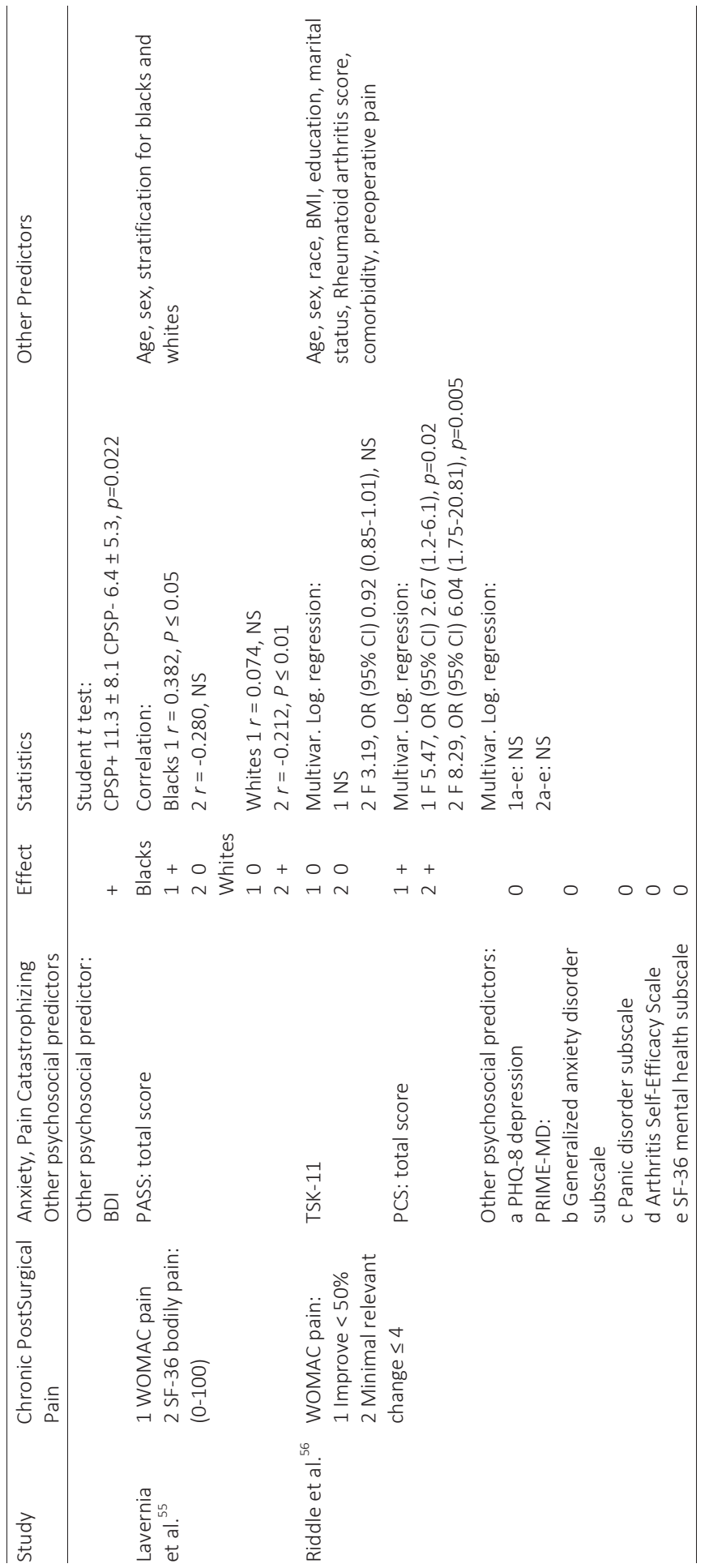




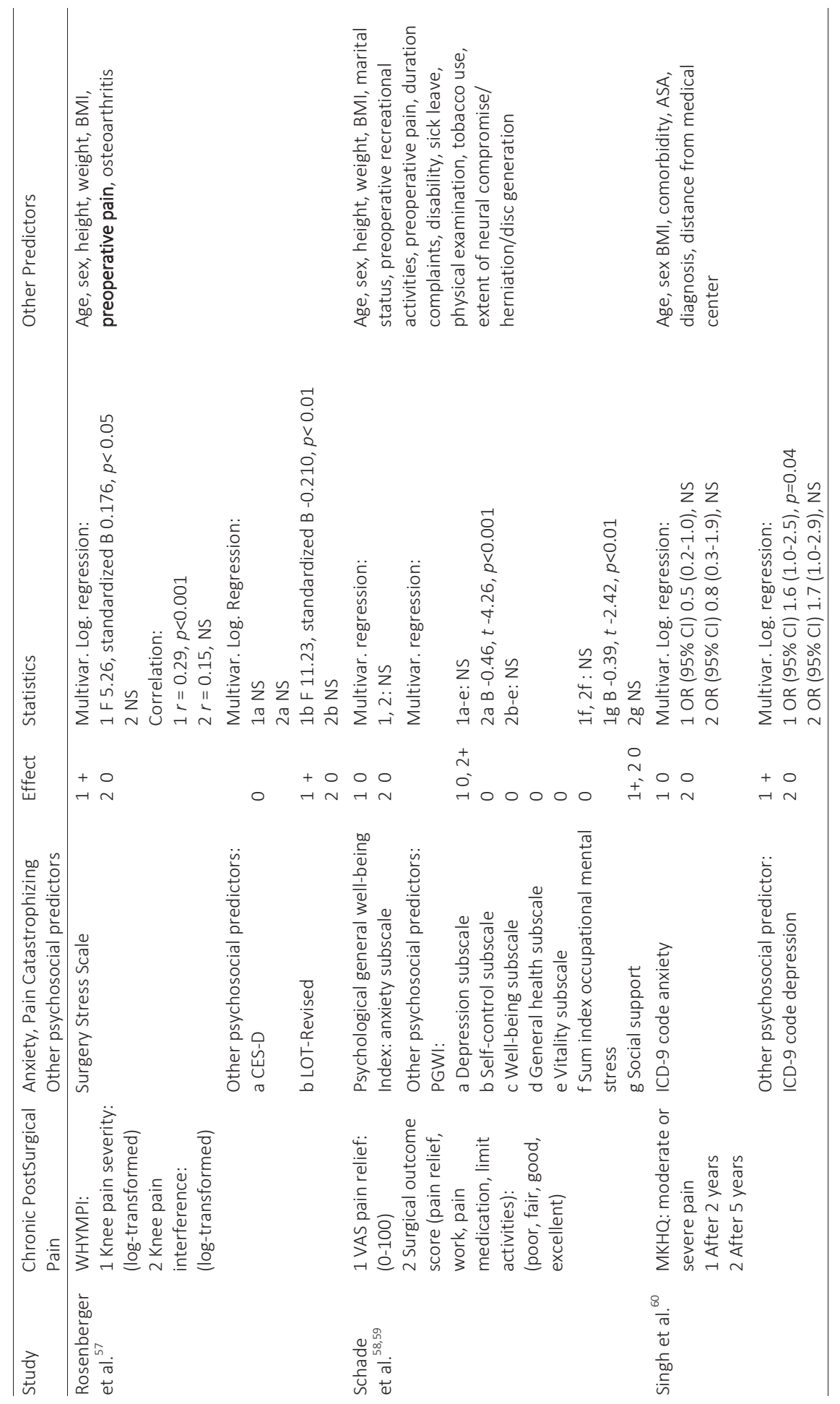




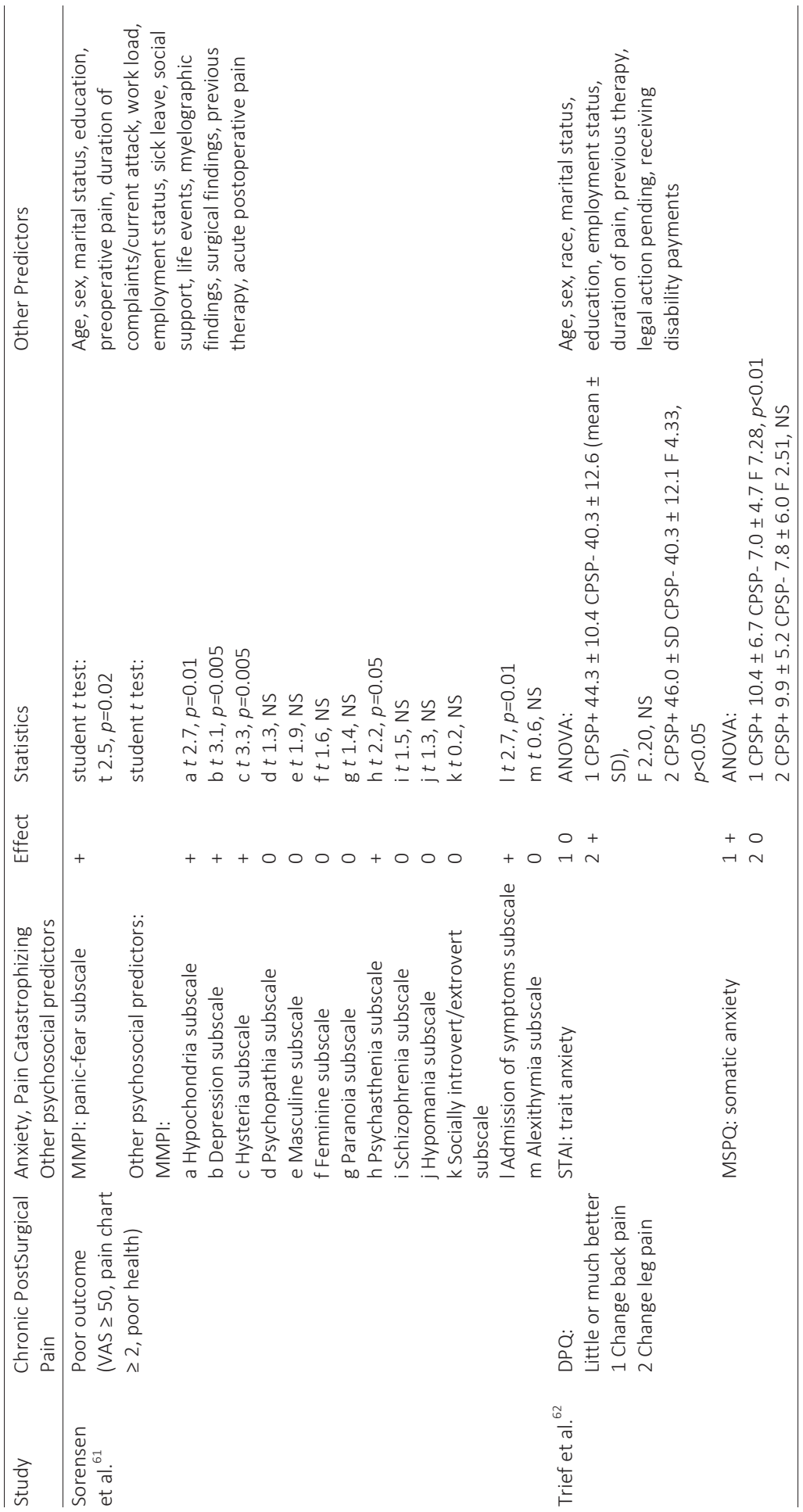




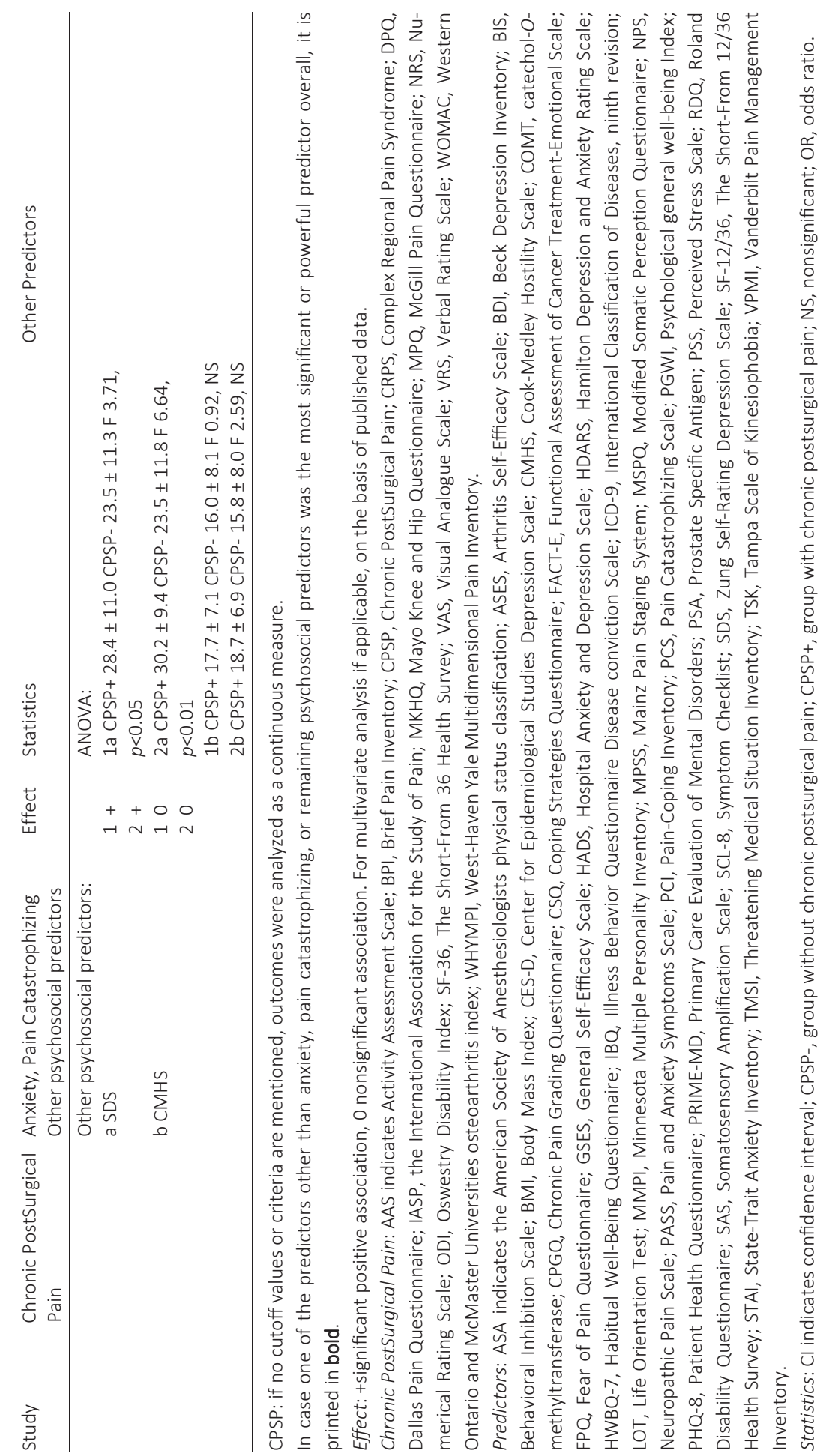




\section{Quality of evidence}

Overall, the quality of studies was relatively high. The quality scores of the selected studies were $5(10 \%),{ }^{19,40,45} 6(42 \%),{ }^{36,38,39,44,46-48,52,54,55,60,61} 7(17 \%),{ }^{37,43,49,53,58}$ or 8 $(31 \%)$, 1,28,35,42,50,51,56,57,62 respectively (Table 1$)$. Agreement between the 2 raters was substantial: the weighted quadratic $\mathrm{k}$ was 0.67 [SD 0.14; 95\% confidence interval (CI), $0.40-0.95)$. The $\mathrm{k}$ scores per item ranged from 0.35 to 1.00 . The lowest values were 0.35 for the item "withdrawal described" and 0.44 for the item "sample representative." A sensitivity analysis revealed that the quality of the selected studies was not affected by publication date (before/after 2008; $\chi^{2} 0.94, d f 3, p=0.82$ ) or by categorization of surgery (musculoskeletal vs. remaining types of surgery; $\chi^{2} 1.25, d f 3, p=0.74$ ). With regard to the relationship between study quality and the reported effects of the predictors, no statistically significant differences were found (studies reporting any significant predictor vs. no significant predictor; $\chi^{2} 1.57, d f 3, p=0.67$ ).

Retrospective studies were excluded. In one of the included studies, selection bias could have played a role. Preoperative anxiety was based on assessment of the ICD-9 anxiety code in the patient file. The author stated that this might have led to underreporting of anxiety. For 6 of the 29 included studies, it was not possible to determine whether the study population was representative of the whole surgical population (eg, single surgical center or only patients of 1 surgeon included). A further 16 (55\%) studies had a sample size of $<100$ participants. Attrition was $>30 \%$ or could not be determined in 2 studies. Confounding variables were adjusted by multivariate analyses in 16 studies; in the remaining studies, bivariate analysis was used or the correction method used to adjust for confounding was unclear.

\section{Measurement of predictors and outcomes}

A total of 14 different instruments were used to assess psychological predictors: (in alphabetical order) Coping Strategies Questionnaire (CSQ, subscale pain catastrophizing); Fear of Pain Questionnaire (FPQ III); Hospital Anxiety and Depression Scale (HADS, subscale anxiety); ICD-9 code anxiety; Minnesota Multiple Personality Inventory [MMPI, subscale panic-fear (PF)]; Modified Somatic Perception Questionnaire (MSPQ, subscale somatic anxiety); Pain and Anxiety Symptoms Scale (PASS); Pain Catastrophizing Scale (PCS); Psychological general well-being index (subscale anxiety); State-Trait Anxiety Inventory (STAI); Surgery Stress Scale; Surgical Fear Questionnaire; Tampa Scale of Kinesiophobia (TSK); the Visual Analogue Scale (VAS anxiety).

A total of 21 different instruments were used to capture pain-related outcomes: Activity Assessment Scale (AAS, pain-related impairment); Brief Pain Inventory [BPI; the pain intensity Numerical Rating Scale (NRS) scores]; Clinical Overall Score (COS; an aggregate measure of pain intensity by VAS, clinical examination, the Oswestry Disability Index, and analgesic use); CPSP presence (Macrae criteria); Dallas Pain Questionnaire 
(back pain, leg pain); International Association for the Study of Pain (IASP) criteria for Complex Regional Pain Syndrome (CRPS); Mayo Knee and Hip Questionnaire; McGill Pain Questionnaire (MPQ, pain rating index); Pain intensity/change by Likert or Visual Analogue Scale (VAS) or Verbal Rating Scale (VRS); Pain relief (VAS); Pelvic pain affecting daily life (Likert); Phantom Phenomena Questionnaire (intensity by VAS, presence yes/no); Poor outcome [aggregate score of pain intensity (VAS), pain chart score, poor health]; Presence of any breast surgery-related pain (yes/no); Retrospective report of increased or new pain due to surgery; Satisfaction with surgical treatment (content/discontent); SF-36 (subscale bodily pain); Surgical outcome score (aggregate score of pain relief, return to work, medication use, limitation of physical activities); Western Ontario and McMaster Universities (WOMAC) Osteoarthritis index; and West-Haven Yale Multidimensional Pain Inventory (MPI).

\section{Predictors of chronic pain after surgery}

In 16 of the 29 studies (55\%), preoperative anxiety or pain catastrophizing was significantly associated with higher rates of CPSP. In all cases, higher levels of anxiety or pain catastrophizing predicted worse outcome. However, in 12 out of these 16 studies using multiple predictor or outcome measures, a significant association was not uniformly found across all measures or analyses. Reported effect sizes varied across studies, but were generally small to moderate (all correlations <0.5; ORs ranged from 1.90 to 6.04). Thirteen studies did not find any association between preoperative anxiety or pain catastrophizing and CPSP (Tables 1 and 2).

Table 3 shows the number of studies that reported a statistically significant effect of a specific predictor on any pain-related outcome. To obtain more homogeneity in the predictors assessed, we divided them into 3 groups: general anxiety measures, painrelated anxiety measures, and pain catastrophizing. Remarkably, pain-related anxiety seems to be less predictive compared with the general anxiety measures and pain catastrophizing, respectively. This seems to be mainly due to an absence of association for the TSK. 


\section{Chapter 2}

Table 3. Predictors: statistical significance per total studies

\begin{tabular}{|c|c|c|}
\hline References & Predictor & $\begin{array}{l}\text { Significant Effect } \\
\text { (Total Studies) }\end{array}$ \\
\hline \multicolumn{3}{|l|}{ Anxiety general } \\
\hline Aasvang and colleagues ${ }^{35,37-40,50}$ & HADS (subscale anxiety) & $3(6)$ \\
\hline Katz and colleagues ${ }^{19,45,46,49,52,54,62}$ & STAI (trait) & $3(7)$ \\
\hline Katz and colleagues ${ }^{19,43,51,54}$ & STAI (state) & $2(4)$ \\
\hline Singh and Lewallen ${ }^{60}$ & ICD-9 & $0(1)$ \\
\hline Schade et al. ${ }^{58}$ & Psychological well-being (subscale anxiety) & $0(1)$ \\
\hline Trief et al. ${ }^{62}$ & MSPQ (subscale anxiety) & $1(1)$ \\
\hline Sorensen et al. ${ }^{61}$ & MMPI (panic-fear scale) & $1(1)$ \\
\hline \multirow[t]{2}{*}{ Hickey et al. ${ }^{40}$} & VAS (anxiety) & $0(1)$ \\
\hline & Sub total & $10(22)$ \\
\hline \multicolumn{3}{|l|}{ Anxiety pain related } \\
\hline den Boer and colleagues ${ }^{28,49,53,56}$ & TSK & $0(4)$ \\
\hline Lavernia et al. ${ }^{55}$ & PASS (total) & $1(1)$ \\
\hline Peters et al. ${ }^{1,42}$ & Surgical fear & $1(2)$ \\
\hline Rosenberger et al. ${ }^{57}$ & Surgery stress scale & $1(1)$ \\
\hline \multirow[t]{2}{*}{ George et al. ${ }^{49}$} & Fear of pain questionnaire & $0(1)$ \\
\hline & Sub total & $3(9)$ \\
\hline \multicolumn{3}{|l|}{ Catastrophizing } \\
\hline Peters and colleagues ${ }^{1,35,42,48,49,56}$ & PCS & $3(6)$ \\
\hline \multirow[t]{3}{*}{ Brandsborg and colleagues ${ }^{36,44,47,53}$} & $\mathrm{CSQ}$ & $2(4)$ \\
\hline & Sub total & $5(10)$ \\
\hline & Total & $18(41)$ \\
\hline
\end{tabular}

Any statistically significant effect (one or more times counts for one) of each predictor per study versus total number of studies using that predictor.

CSQ indicates Coping Strategies Questionnaire; HADS, Hospital Anxiety and Depression Scale; ICD-9, International Classification of Diseases, ninth revision; MMPI, Minnesota Multiple Personality Inventory; MSPQ, Modified Symptom Perception Questionnaire; PASS, Pain Anxiety Symptoms Scale; PCS, Pain Catastrophizing Scale; STAI, State-Trait Anxiety Inventory; TSK, Tampa Scale of Kinesiophobia; VAS, Visual Analog Scale.

Next, we assessed whether there were differences in outcome between studies addressing musculoskeletal surgery versus other types of surgery. There was a trend showing an increased proportion of statistically significant predictors in studies of musculoskeletal surgery $(67 \%)$ compared with the studies investigating other types of surgery (36\%, $\left.\chi^{2} 2.54, d f 1, p=0.11\right)$. Stratification for sample size is shown in Table 4. The proportion of studies revealing statistically significant predictors was $69 \%$ for studies with $>100$ participants compared with $44 \%$ for the smaller studies $\left(\chi^{2} 1.88\right.$, df 1 , $p=0.17)$. The proportion of statistically significant predictors was similar for the higher quality studies (scores 7 to 8 ) (57\%) compared with studies with intermediate quality scores 5 to $6\left(53 \%, \chi^{2} 0.04, d f 1, p=0.84\right)$. The length of postoperative follow-up, 3 to 12 months compared with 12 months or more, did not yield a significantly different pro- 
portion of studies reporting a significant association between predictor and outcome (respectively, 53\% and 57\%, $\chi^{2} 0.04, d f 1, p=0.84$ ). For studies using bivariate versus multivariate analysis, the proportions were $62 \%$ and $50 \%$, respectively $\left(\chi^{2} 0.39, d f 1\right.$, $p=0.53)$.

Table 4. Predictors: statistical significance and sample size

\begin{tabular}{ll}
\hline Sample size & $\begin{array}{l}\text { Significant studies } \\
\text { (Total studies) }\end{array}$ \\
\hline $\mathrm{N} \leq 100$ & $7(16)$ \\
$\mathrm{N}>100$ & $9(13)$ \\
Total & $16(29)$ \\
\hline
\end{tabular}

Studies reporting any statistically significant effect versus total number of studies per sample size.

For the meta-analysis, pooled ORs were calculated on the basis of 15 studies including 5046 patients. Five studies made use of multivariate analysis ${ }^{1,35,49,56,60}$ and 10 were based on bivariate analysis. ${ }^{36,38-40,42,44,47,53,57,61}$ Table 5 presents pooled ORs with their $\mathrm{Cl}$ and a measure for between-study variation (heterogeneity). Overall, anxiety and catastrophizing seemed to be significant predictors of CPSP. Depending on the (sub)group and the maximum or the minimum effect scenario, pooled ORs varied between 1.11 (95\% Cl, 0.82-1.50) and 2.71 (95\% Cl, 1.40-5.24), with a total OR of 2.1 (95\% $\mathrm{Cl}, 1.49-2.95)$ for the maximum effect scenario and $1.55(95 \% \mathrm{Cl}, 1.10-2.20)$ for the minimum effect scenario. Because the OR of 1 study $^{49}$ included an interaction effect of catastrophizing with the COMT gene, the analysis was repeated excluding these data. Although ORs were reduced slightly, the direction of the effects did not change, except for the magnitude of the total OR, minimum effect scenario: 1.32 ( $95 \% \mathrm{Cl}, 0.98$ to 1.77). The pooled ORs confirmed the trend from the vote counting procedure that painrelated anxiety measures were less predictive for CPSP than either pain catastrophizing or pain-related anxiety. Moreover, the ORs also suggest the relative superiority of pain catastrophizing as a predictor. Finally, the trend showing that anxiety and catastrophizing are most predictive for CPSP in musculoskeletal surgery was confirmed by the pooled ORs according to the maximum effect scenario but not by the minimum effect scenario. 
Table 5. Pooled effect sizes

\begin{tabular}{|c|c|c|c|c|c|c|c|c|}
\hline \multirow{3}{*}{$\begin{array}{l}\text { (Sub-)Group } \\
\text { General anxiety } \\
35,38-40,60,61\end{array}$} & \multicolumn{4}{|c|}{ Maximum effect scenario } & \multicolumn{4}{|c|}{ Minimum effect scenario } \\
\hline & \multirow{2}{*}{$\begin{array}{l}\text { Pooled } \\
\text { OR } \\
1.76\end{array}$} & \multirow{2}{*}{$\begin{array}{l}95 \% \mathrm{Cl} \\
1.07-2.90\end{array}$} & \multicolumn{2}{|c|}{ Test for heterogeneity } & \multirow{2}{*}{$\begin{array}{l}\text { Pooled } \\
\text { OR } \\
1.56\end{array}$} & \multirow{2}{*}{$\begin{array}{l}95 \% \mathrm{Cl} \\
0.76-3.24\end{array}$} & \multicolumn{2}{|c|}{ Test for heterogeneity } \\
\hline & & & $5.53 d f 5$ & $p=0.355$ & & & $11.13 d f 5$ & $p=0.049$ \\
\hline $\begin{array}{l}\text { Anxiety pain } \\
\text { related }^{1,42,53,56,57}\end{array}$ & 1.25 & $0.87-1.79$ & $10.83 d f 4$ & $p=0.029$ & 1.11 & $0.82-1.50$ & $8.19 d f 4$ & $p=0.085$ \\
\hline $\begin{array}{l}\text { Catastrophizing } \\
35,36,42,44,47,49,53,56\end{array}$ & 2.37 & $1.32-4.28$ & $20.94 d f 7$ & $p=0.004$ & 13 & $1.26-3.59$ & $18.31 d f 7$ & $p=0.011$ \\
\hline $\begin{array}{l}\text { Various types } \\
\text { of surgery } \\
1,35,36,38-40,42,44\end{array}$ & 1.57 & $1.18-2.10$ & $6.83 d f 7$ & $p=0.447$ & 1.57 & $1.13-2.16$ & $8.06 d f 7$ & $p=0.328$ \\
\hline $\begin{array}{l}\text { Musculoskeletal } \\
47,49,53,56,57,60,61\end{array}$ & 2.71 & $1.40-5.24$ & $15.46 d f 6$ & $p=0.017$ & 1.51 & $0.82-2.80$ & $28.16 d f 6$ & $p<0.001$ \\
\hline Total & 2.10 & $1.49-2.95$ & $25.37 d f 14$ & $p=0.031$ & 1.55 & $1.10-2.20$ & $45.89 d f 14$ & $p<0.001$ \\
\hline
\end{tabular}

Maximum effect scenario: highest OR per (sub-)group; minimum effect scenario: lowest OR per (sub-)group. Test for heterogeneity: $\chi^{2}$.

$95 \% \mathrm{Cl}$ indicates $95 \%$ confidence interval; OR, odds ratio.

\section{DISCUSSION}

This systematic review identified 29 studies that examined the relationship between preoperative psychological factors and the risk of persistent, chronic pain after surgery. In 16 out of 29 studies (55\%), at least one of the measures of preoperative anxiety or pain catastrophizing showed a significant positive association with CPSP. The remaining 13 studies (45\%) failed to detect a relationship between anxiety or pain catastrophizing and CPSP. No studies reported a reversed association, that is a decreased risk of CPSP with high levels of anxiety or lower levels of anxiety predicting CPSP. Overall effect sizes on the basis of 15 studies varied between pooled OR $1.55(95 \% \mathrm{Cl}, 1.10-2.20$, minimum effect scenario) and pooled OR 2.10 (95\% Cl, 1.49-2.95, maximum effect scenario).

Previous systematic reviews by Huber and Lautenbacher, ${ }^{8}$ which included 6 empirical papers, and Hinrichs-Rocker et al., ${ }^{25}$ which included 7 empirical papers examining the relationship between preoperative anxiety and CPSP, both concluded that there is inconclusive evidence for a predictive relationship. Our systematic review was more comprehensive in that we were able to identify no less than 29 empirical papers investigating the relationship between preoperative anxiety or pain catastrophizing and CPSP. Moreover, we were able to perform a meta-analysis. Although there was no consistent relationship between anxiety or pain catastrophizing and CPSP among the 29 papers, a small majority of studies demonstrated a statistically significant positive association, with no study reporting a reversed effect. It is noteworthy that the results were not related to overall study quality, but that studies with larger sample sizes more often yielded significant relationships. The meta-analysis on a subset of the papers provided 
additional evidence for an association between preoperative anxiety or catastrophizing and CPSP, yielding effect sizes (pooled ORs) varying between 1.55 and 2.10. Therefore, we propose that there is at least moderate evidence that preoperative anxiety and pain catastrophizing play a role in the development of chronic pain after surgery.

A secondary aim of this review was to examine whether the association between anxiety and pain catastrophizing is more evident in patients undergoing musculoskeletal surgery compared with other types of surgery. Our results show that the proportion of studies showing a significant association was indeed larger and the maximum effect ORs were higher for the subgroup of musculoskeletal surgery patients. It is important to note that CPSP in these patients may be a continuation of preexisting pain instead of new onset pain after surgery as in the definition proposed by Macrae. ${ }^{22}$ Thus, it may be speculated that anxiety and pain catastrophizing play a more prominent role in nonrecovery from (often longstanding) pain than inciting new pain.

We also compared the predictive utility of instruments assessing different anxietyrelated concepts. We categorized the anxiety measures into 3 main groups: general anxiety, pain-related anxiety, and (pain) catastrophizing. There did not seem to be an overall superior predictive value of 1 of the 3 groups. However, on the basis of our meta-analysis, catastrophizing was the most consistent predictor. Studies by George et al. ${ }^{49}$ and Riddle et al. ${ }^{56}$ reported that when anxiety and pain catastrophizing were entered into a regression model simultaneously, only pain catastrophizing was an independent predictor of CPSP. However, the opposite effect was found by Peters et al., ${ }^{1}$ who reported that anxiety instead of pain catastrophizing remained a significant independent predictor after multivariate analysis. Although anxiety and pain catastrophizing are presumed to measure 2 separate constructs (ie, an affective reaction vs. beliefs about pain), they are considerably correlated. Therefore, when entering them simultaneously in one analysis, one may take precedence over the other because of shared explained variance.

One striking finding was the absence of the predictive utility of the TSK across studies. This instrument was developed to capture fear of movement in patients with chronic musculoskeletal pain and not specifically for surgical patients. Although the TSK was used in studies of knee, shoulder, and lumbar surgery, it was not independently predictive of CPSP. It may be proposed that fear of movement is more predictive of painrelated disability or activity-related outcomes than for pain per se. However, 3 of the 4 studies using the TSK also included a disability questionnaire, and only 1 study found that preoperative TSK scores were predictive of continued disability. ${ }^{28}$

Concerning the quality of evidence, several remarks can be made. First, the quality scores of the included articles were generally high, ranging from a total score of between 5 and 8 on a 0 to 8 scale. Study quality had no effect on the results. Second, in general, for predictors and outcome measures, validated or at least well-described measures were used. All studies used a prospective design. Third, the main bias in the review process was related to the heterogeneity in the measurement and reporting of 
predictors, outcome measures, and in statistical analyses, making comparison of results difficult. Therefore, the pooled results should be interpreted with caution. The postoperative follow-up interval also varied between studies but was at least 3 months for every included study. Fourth, the quality of evidence could have been improved, particularly if larger study samples were used, allowing correction for confounding by multivariate analysis. Fifth, there may have been an underreport of anxiety in some studies. For instance, in the study by Singh and Lewallen, ${ }^{60}$ anxiety was based on an ICD-9 anxiety diagnosis extracted from the patient file. Moreover, 2 studies excluded patients if they were on current treatment with anxiolytic drugs. ${ }^{45,46}$ Thus, a decreased prevalence of preoperative anxiety may have occurred in certain study populations, with a negative effect on the power to detect a relationship. Finally, none of the included studies incorporated an intervention aimed at reduction of preoperative anxiety or pain catastrophizing.

\section{Conclusions}

This updated systematic review and meta-analysis demonstrates that there is evidence that anxiety plays a role in the occurrence of chronic pain after surgery. Fifty-five percent of studies reported a positive association between preoperative anxiety or pain catastrophizing and CPSP and no studies reported a negative association. The metaanalysis on a subset of studies yielded pooled ORs in the range of 1.55 to 2.10 for the association between anxiety/catastrophizing and CPSP. The effect seemed to be somewhat more evident and consistent in musculoskeletal surgery, where $67 \%$ of the studies showed a significant positive association versus $36 \%$ in the remaining studies of other types of surgery. The results also suggest that the predictive value of pain catastrophizing is more consistent compared with general anxiety and pain-related fear. The TSK seems to be less appropriate for the prediction of chronic pain after surgery.

\section{Implications for practice}

It is premature to recommend widespread implementation of preoperative screening of anxiety as a predictor of CPSP. Further research is required to establish the most sensitive psychological predictors of CPSP. Then, interventions targeted toward reducing preoperative anxiety might be a next step in the prevention of CPSP.

\section{Implications for research}

There is an urgent need for larger prospective studies allowing more powerful multivariate analyses to establish which anxiety-related construct is the most important and which assessment instrument is the most suitable for predicting CPSP. Results should be clearly presented, including intercorrelation between used predictors, uniform outcome 
measures, and facilitating statistical pooling. A taskforce should be assembled to establish one or a strictly limited number of "standard" anxiety measures that preferably should be included in every study to facilitate future comparison of results.

\section{ACKNOWLEDGMENTS}

The authors thank Veronique Timmer for her participation in the quality assessment of the included articles during her scientific traineeship at our department. 


\section{REFERENCES}

1. Peters ML, Sommer M, de Rijke JM, et al. Somatic and psychologic predictors of long-term unfavorable outcome after surgical intervention. Ann Surg. 2007;245:487-494.

2. Gramke HF, de Rijke JM, van Kleef $M$, et al. Predictive factors of postoperative pain after day-case surgery. Clin J Pain. 2009;25:455-460.

3. Gramke HF, de Rijke JM, van Kleef M, et al. The prevalence of postoperative pain in a cross-sectional group of patients after day-case surgery in a university hospital. Clin J Pain. 2007;23:543-548.

4. Perkins FM, Kehlet $\mathrm{H}$. Chronic pain as an outcome of surgery. A review of predictive factors. Anesthesiology. 2000;93:1123-1133.

5. Sommer M, Geurts JW, Stessel B, et al. Prevalence and predictors of postoperative pain after ear, nose, and throat surgery. Arch Otolaryngol Head Neck Surg. 2009;135:124-130.

6. Ip HY, Abrishami A, Peng PW, et al. Predictors of postoperative pain and analgesic consumption: a qualitative systematic review. Anesthesiology. 2009;111:657-677.

7. Sommer M, de Rijke JM, van Kleef $M$, et al. Predictors of acute postoperative pain after elective surgery. Clin J Pain. 2010;26:87-94.

8. Huber C, Lautenbacher S. The relevance of psychological variables in postoperative pain - Psychological variables and postoperative pain. Anasth Intensivmed. 2008;49:1-16.

9. Munafo MR, Stevenson J. Anxiety and surgical recovery. Reinterpreting the literature. J Psychosom Res. 2001;51:589-596.

10. Vaughn F, Wichowski H, Bosworth G. Does preoperative anxiety level predict postoperative pain? Aorn J. 2007;85:589-604.

11. Spielberger CD. Assessment of state and trait anxiety: conceptual and methodological issues. South Psychol. 1985;2:6-16.

12. Granot M, Ferber SG. The roles of pain catastrophizing and anxiety in the prediction of postoperative pain intensity: a prospective study. Clin J Pain. 2005;21:439-445.

13. Papaioannou M, Skapinakis $P$, Damigos $D$, et al. The role of catastrophizing in the prediction of postoperative pain. Pain Med. 2009;10:1452-1459.

14. Pavlin DJ, Sullivan MJ, Freund PR, et al. Catastrophizing: a risk factor for postsurgical pain. Clin J Pain. 2005;21:83-90.

15. Quartana PJ, Campbell CM, Edwards RR. Pain catastrophizing: a critical review. Expert Rev Neurother. 2009;9:745-758.

16. Sullivan MJ, Thorn B, Rodgers W, et al. Path model of psychological antecedents to pain experience: experimental and clinical findings. Clin J Pain. 2004;20:164-173.

17. Sullivan MJLB, Bishop SR, Pivik J. The Pain Catastrophizing Scale: development and validation. Psychol Assess. 1995; 4: 524-532.

18. Crombie IK, Davies HT, Macrae WA. Cut and thrust: antecedent surgery and trauma among patients attending a chronic pain clinic. Pain. 1998;76:167-171.

19. Katz J, Jackson M, Kavanagh BP, et al. Acute pain after thoracic surgery predicts long-term postthoracotomy pain. Clin J Pain. 1996;12:50-55.

20. Kehlet H, Bay-Nielsen M, Kingsnorth A. Chronic postherniorrhaphy pain-a call for uniform assessment. Hernia. 2002;6: 178-181.

21. Classification of chronic pain. Descriptions of chronic pain syndromes and definitions of pain terms. Prepared by the International Association for the Study of Pain, Subcommittee on Taxonomy. Pain. 1986;3:S1-226.

22. Macrae WA. Chronic pain after surgery. Br J Anaesth. 2001;87:88-98.

23. Kehlet $\mathrm{H}$, Jensen TS, Woolf CJ. Persistent postsurgical pain: risk factors and prevention. Lancet. 2006;367:1618-1625.

24. Katz J, Seltzer Z. Transition from acute to chronic postsurgical pain: risk factors and protective factors. Expert Rev Neurother. 2009;9:723-744. 
25. Hinrichs-Rocker A, Schulz K, Jarvinen I, et al. Psychosocial predictors and correlates for chronic postsurgical pain (CPSP) - a systematic review. Eur J Pain. 2009;13:719-730.

26. Leeuw M, Goossens ME, Linton SJ, et al. The fear-avoidance model of musculoskeletal pain: current state of scientific evidence. J Behav Med. 2007;30:77-94.

27. Vancleef LM, Vlaeyen JW, Peters ML. Dimensional and componential structure of a hierarchical organization of pain-related anxiety constructs. Psychol Assess. 2009;21:340-351.

28. den Boer JJ, Oostendorp RA, Beems T, et al. Continued disability and pain after lumbar disc surgery: the role of cognitive-behavioral factors. Pain. 2006;123:45-52.

29. den Boer JJ, Oostendorp RA, Beems T, et al. A systematic review of bio-psychosocial risk factors for an unfavourable outcome after lumbar disc surgery. Eur Spine J. 2006;15:527-536.

30. Celestin J, Edwards RR, Jamison RN. Pretreatment psychosocial variables as predictors of outcomes following lumbar surgery and spinal cord stimulation: a systematic review and literature synthesis. Pain Med. 2009;10:639-653.

31. Joanna Briggs Institute. Joanna Briggs Insitute Reviewers' Manual: 2008 edition. 2008 ed. Adelaide: The Joanna Briggs Institute; 2008.

32. Downs SH, Black N. The feasibility of creating a checklist for the assessment of the methodological quality both of randomised and non-randomised studies of health care interventions. J Epidemiol Community Health. 1998;52:377-384.

33. Cohen J. Weighted kappa: nominal scale agreement with provision for scaled disagreement or partial credit. Psychol Bull. 1968;70:213-220.

34. von Elm E, Altman DG, Egger M, et al. The Strengthening the Reporting of Observational Studies in Epidemiology (STROBE) statement: guidelines for reporting observational studies. J Clin Epidemiol. 2008;61:344-349.

35. Aasvang EK, Gmaehle E, Hansen JB, et al. Predictive risk factors for persistent postherniotomy pain. Anesthesiology. 2010;112:957-969.

36. Brandsborg B, Dueholm M, Nikolajsen L, et al. A prospective study of risk factors for pain persisting 4 months after hysterectomy. Clin J Pain. 2009;25:263-268.

37. Ene KW, Nordberg G, Johansson FG, et al. Pain, psychological distress and health-related quality of life at baseline and 3 months after radical prostatectomy. BMC Nurs. 2006;5:8.

38. Gerbershagen HJ, Dagtekin O, Rothe $\mathrm{T}$, et al. Risk factors for acute and chronic postoperative pain in patients with benign and malignant renal disease after nephrectomy. Eur J Pain. 2009;13:853-860.

39. Gerbershagen HJ, Ozgur E, Dagtekin O, et al. Preoperative pain as a risk factor for chronic post-surgical pain - six month follow-up after radical prostatectomy. Eur J Pain. 2009;13:1054-1061.

40. Hickey OT, Burke SM, Hafeez P, et al. Severity of acute pain after breast surgery is associated with the likelihood of subsequently developing persistent pain. Clin J Pain. 2010;26:556-560.

41. Kavanagh BP, Katz J, Sandler AN, et al. Multimodal analgesia before thoracic surgery does not reduce postoperative pain. Br J Anaesth. 1994;73:184-189.

42. Peters ML, Sommer M, van Kleef M, et al. Predictors of physical and emotional recovery 6 and 12 months after surgery. Br J Surg. 2010;97:1518-1527.

43. Poleshuck EL, Katz J, Andrus $\mathrm{CH}$, et al. Risk factors for chronic pain following breast cancer surgery: a prospective study. J Pain. 2006;7:626-634.

44. Richardson C, Glenn S, Horgan M, et al. A prospective study of factors associated with the presence of phantom limb pain six months after major lower limb amputation in patients with peripheral vascular disease. J Pain. 2007;8:793-801.

45. Brander VA, Stulberg SD, Adams AD, et al. Predicting total knee replacement pain: a prospective, observational study. Clin Orthop Relat Res. 2003;416:27-36.

46. Brander V, Gondek S, Martin E, et al. Pain and depression influence outcome 5 years after knee replacement surgery. Clin Orthop Relat Res. 2007;464:21-26.

47. Edwards RR, Haythornthwaite JA, Smith MT, et al. Catastrophizing and depressive symptoms as prospective predictors of outcomes following total knee replacement. Pain Res Manag. 2009;14:307-311. 


\section{Chapter 2}

48. Forsythe ME, Dunbar MJ, Hennigar AW, et al. Prospective relation between catastrophizing and residual pain following knee arthroplasty: two-year follow-up. Pain Res Manage. 2008;13:335-341.

49. George SZ, Wallace MR, Wright TW, et al. Evidence for a biopsychosocial influence on shoulder pain: pain catastrophizing and catechol-O-methyltransferase (COMT) diplotype predict clinical pain ratings. Pain. 2008;136:53-61.

50. Graver V, Ljunggren AE, Malt UF, et al. Can psychological traits predict the outcome of lumbar disc surgery when anamnestic and physiological risk factors are controlled for? Results of a prospective cohort study. J Psychosom Res. 1995;39:465-476.

51. de Groot KI, Boeke S, van den Berge HJ, et al. Assessing short- and long-term recovery from lumbar surgery with pre-operative biographical, medical and psychological variables. Brit J Health Psychol. 1997;2:229-243.

52. Harden RN, Bruehl S, Stanos S, et al. Prospective examination of pain-related and psychological predictors of CRPS-like phenomena following total knee arthroplasty: a preliminary study. Pain. 2003;106:393400.

53. Johansson AC, Linton SJ, Rosenblad A, et al. A prospective study of cognitive behavioural factors as predictors of pain, disability and quality of life one year after lumbar disc surgery. Disabil Rehabil. 2010;32:521-529.

54. Kjellby-Wendt G, Styf JR, Carlsson SG. The predictive value of psychometric analysis in patients treated by extirpation of lumbar intervertebral disc herniation. J Spinal Disord. 1999;12:375-379.

55. Lavernia CJ, Alcerro JC, Rossi MD. Fear in arthroplasty surgery: the role of race. Clin Orthop Relat Res. 2009;468: 547-554.

56. Riddle DL, Wade JB, Jiranek WA, et al. Preoperative pain catastrophizing predicts pain outcome after knee arthroplasty. Clin Orthop Relat Res. 2010;468:798-806.

57. Rosenberger PH, Kerns R, Jokl P, et al. Mood and attitude predict pain outcomes following arthroscopic knee surgery. Ann Behav Med. 2009;37:70-76.

58. Schade V, Semmer N, Main CJ, et al. The impact of clinical, morphological, psychosocial and work-related factors on the outcome of lumbar discectomy. Pain. 1999;80:239-249.

59. Boos N, Rieder R, Schade V, et al. 1995 Volvo Award in clinical sciences. The diagnostic accuracy of magnetic resonance imaging, work perception, and psychosocial factors in identifying symptomatic disc herniations. Spine. 1995;20: 2613-2625.

60. Singh JA, Lewallen D. Age, gender, obesity, and depression are associated with patient-related pain and function outcome after revision total hip arthroplasty. Clin Rheumatol. 2009; 28:1419-1430.

61. Sorensen LV, Mors O, Skovlund O. A prospective study of the importance of psychological and social factors for the outcome after surgery in patients with slipped lumbar disk operated upon for the first time. Acta Neurochir (Wien). 1987;88:119-125.

62. Trief PM, Grant W, Fredrickson B. A prospective study of psychological predictors of lumbar surgery outcome. Spine. 2000;25:2616-2621. 


\section{APPENDIX}

Checklist quality of observational studies

\begin{tabular}{|c|c|c|c|c|}
\hline & Checklist quality of observational studies for systematic review & Yes & No & $\begin{array}{l}\text { Unable to } \\
\text { determine }\end{array}$ \\
\hline & Author (year): & $(1)$ & $(0)$ & (0) \\
\hline 1 & $\begin{array}{l}\text { Is the sample representative of patients in the population as a whole? } \\
\text { (external validity) }\end{array}$ & & & \\
\hline 2 & $\begin{array}{l}\text { If any of the results were based on data dredging (retrospective), was } \\
\text { this made clear? } \\
\text { (internal validity/bias) }\end{array}$ & & & \\
\hline 3 & $\begin{array}{l}\text { Were the main predictors used accurate: reliable and valid? } \\
\text { (internal validity/bias) }\end{array}$ & & & \\
\hline 4 & $\begin{array}{l}\text { Were the main outcome measures used accurate: reliable and valid? } \\
\text { (internal validity/bias) }\end{array}$ & & & \\
\hline 5 & $\begin{array}{l}\text { Were the statistical tests used to assess the main outcomes } \\
\text { appropriate? } \\
\text { (internal validity/bias) }\end{array}$ & & & \\
\hline 6 & $\begin{array}{l}\text { Were the outcomes of people who withdrew described and included in } \\
\text { the analysis? } \\
\text { (internal validity/selection bias) }\end{array}$ & & & \\
\hline 7 & $\begin{array}{l}\text { Was there adequate adjustment for confounding in the analyses from } \\
\text { which the main findings are drawn? } \\
\text { (internal validity/confounding) }\end{array}$ & & & \\
\hline \multirow[t]{2}{*}{8} & $\begin{array}{l}\text { Is the sample size }>100 ? \\
\text { (power) }\end{array}$ & & & \\
\hline & Total score & & & \\
\hline
\end{tabular}

Checklist quality of studies for systematic review

Criteria Explanation (Based on Downs \& Black ${ }^{32}$ )

1 Is the sample representative The study must identify the source population for patients and describe of patients in the population how the patients were selected. Patients would be representative if they as a whole? (external validity) comprised the entire source population, an unselected sample of consecutive patients, or a random sample.

Random sampling is only feasible where a list of all members of the relevant population exists.

Where a study does not report the proportion of the source population from which the patients are derived, the question should be answered as unable to determine.

2 If any of the results were Any analyses that had not been planned at the outset of the study should based on data dredging be clearly indicated.

(retrospective), was this If no retrospective unplanned subgroup analyses were reported, then made clear? answer yes.

(internal validity/bias) 
Checklist quality of studies for systematic review

Criteria Explanation (Based on Downs \& Black ${ }^{32}$ )

3 Were the main predictors For studies where the predictors are clearly described, the question used accurate: reliable and should be answered yes.

valid? (internal validity/bias) For studies which refer to other work or that demonstrates the predictors are accurate, the question should be answered as yes. For studies were validated instruments were used, the question should be answered yes.

For studies using a self-developed instrument were the psychometric characteristics are clearly described, the question should be answered yes.

In case of more predictors, the predictor used for the main analysis should be accurate.

$4 \quad$ Were the main outcome For studies where the outcome measures are clearly described, the measures used accurate: reliable and valid? (internal validity/bias)

$5 \quad$ Were the statistical tests used to assess the main outcomes appropriate? (internal validity/bias)

6 Were the outcomes of people who withdrew described and included in the analysis? (internal validity/selection bias)

7 Was there adequate question should be answered yes.

For studies which refer to other work or that demonstrates the outcome measures are accurate, the question should be answered as yes.

For studies where validated instruments were used, the question should be answered yes.

For studies using a self-developed instrument were the characteristics of pain are clearly described, the question should be answered yes. In case of more outcomes, the outcome used for the main analysis should be accurate.

The statistical techniques used must be appropriate to the data. For example nonparametric methods should be used for small sample sizes. Where little statistical analysis has been undertaken but where there is no evidence of bias, the question should be answered yes. If the distribution of the data (normal or not) is not described it must be assumed that the estimates used were appropriate and the question should be answered yes.

$\mathrm{N}$ should be at least 10x the number of used predictors.

If the numbers of patients lost to follow-up are not reported, the question should be answered as unable to determine.

If the proportion lost to follow-up was too small to affect the main findings $(<30 \%)$, the question should be answered yes.

In nonrandomized studies if the effect of the main confounders was not adjustment for confounding investigated or confounding was demonstrated but no adjustment was in the analyses from which made in the final analyses the question should be answered as no. the main findings are drawn? (internal validity/ confounding) (power) 


\section{Chapter}

\section{Validation of the Surgical Fear Questionnaire in adult patients waiting for elective surgery}

This chapter is published as: Maurice Theunissen, Madelon L. Peters, Erik G. W.

Schouten, Audrey A. A. Fiddelers, Mark G. A. Willemsen, Patrícia R. Pinto, Hans-Fritz Gramke, Marco A. E. Marcus (2014). Validation of the Surgical Fear Questionnaire in adult patients waiting for elective surgery. PLOS ONE 9(6): e100225. 


\section{ABSTRACT}

Objectives: Because existing instruments for assessing surgical fear seem either too general or too limited, the Surgical Fear Questionnaire (SFQ) was developed. The aim of this study is to assess the validity and reliability of the SFQ.

Methods: Based on existing literature and expert consultation the ten-item SFQ was composed. Data on the SFQ were obtained from 5 prospective studies ( $N=3233)$ in inpatient or day surgery patients. These data were used for exploratory factor analysis (EFA), confirmatory factor analysis (CFA), reliability analysis and validity analysis.

Results: EFA in Study 1 and 2 revealed a two-factor structure with one factor associated with fear of the short-term consequences of surgery (SFQ-S, item 1-4) and the other factor with fear of the long-term consequences of surgery (SFQ-I, item 5-10). However, in both studies two items of the SFQ-I had low factor loadings. Therefore in Study 3 and 4 the two-factor structure was tested and confirmed by CFA in an eight-item version of the SFQ. Across all studies significant correlations of the SFQ with pain catastrophizing, state anxiety, and preoperative pain intensity indicated good convergent validity. Internal consistency (Cronbach's alpha) was between 0.765-0.920 (SFQ-total), 0.766-0.877 (SFQ-S), and 0.628-0.899 (SFQ-I). The SFQ proved to be sensitive to detect differences based on age, sex, education level, employment status and preoperative pain intensity.

Discussion: The SFQ is a valid and reliable eight-item index of surgical fear consisting of two subscales: fear of the short-term consequences of surgery and fear of the longterm consequences. 


\section{INTRODUCTION}

Preoperative or surgical fear is a well recognizable emotional state for many patients waiting for surgery and is a risk factor for major personal and socio-economic burden. Various studies have found that surgical fear is associated with impaired psychosocial and physical recovery, such as increased levels of acute and chronic postoperative pain. $^{1-3}$ Therefore, preoperative assessment of surgical fear could provide essential information for improving perioperative care and could be a first step towards targeted intervention.

Objects of surgical fear can be heterogeneous. Previous studies have listed more than 20 objects of fear, varying from fear of the surgical procedure itself to fear of the anaesthesia, having to undergo blood transfusions, being stung with needles, losing dignity or even dying. ${ }^{4-6}$ Some factors that may influence the reported prevalence of surgical fear are type or impact of planned surgery, time span until surgery, previous experience with surgery, provision of preoperative information about surgical procedure, age and sex. ${ }^{3,5,7-9}$ Also, the instrument used for assessment of fear may influence the reported prevalence.

Only few instruments are available for assessment of surgical fear and most of these are disease specific, such as the Bypass Grafting Fear Scale (BGFS) ${ }^{10}$ and the Surgery Stress Scale (for knee surgery). ${ }^{11}$ Therefore, in many studies, nonspecific instruments have been used such as the Hospital Anxiety and Depression Scale (HADS), ${ }^{12}$ State-Trait Anxiety Inventory (STAI), ${ }^{13}$ or a Visual Analogue Scale (VAS) assessing anxiety. One generic instrument has been developed for preoperative assessment of surgical fear, the six-item Amsterdam Preoperative Anxiety and Information Scale (APAIS). ${ }^{14,15}$ However, this instrument is relatively limited in scope; it includes two items on fear of the anaesthetic procedure and two items on fear of the surgical procedure. The remaining two items asses the need for information rather than fear.

Because existing instruments for assessing surgical fear are either limited in scope, or too general, or too specific and not broadly generalizable to other surgical populations, we developed the Surgical Fear Questionnaire (SFQ). The SFQ has already been used in several studies ${ }^{16-22}$ but formal assessment of its validity and reliability is still lacking. This paper describes the development and psychometric assessment of the SFQ. Similar to the BGFS, ${ }^{10}$ the SFQ aims to be comprehensive enough to cover the most important targets of fear and at the same time concise enough for general use in clinical practice and research. We present data on the construct, content, convergent, and predictive validity as well as the internal consistency of the SFQ. Data from five different studies in which the SFQ was administered to patients one day to one week prior to undergoing inpatient or day surgery are used. Because patients from different clinical populations and different countries are included, this also allows us to test the stability of the SFQ and its factor structure across different subgroups. 


\section{MATERIALS AND METHODS}

\section{Ethics statement}

Study 1, 3, 4, and 5 were approved by the Medical Ethics Committee of Maastricht University Medical Center+, Maastricht, the Netherlands. For Study 2 approval was given by the Medical Ethics Committee of the Centro Hospital do Alto Ave, Guimarães, Portugal. All patients gave written informed consent.

\section{Scale development}

The SFQ was developed to create a tailor made instrument for the assessment of selfreported surgical fear, suitable for general use among all types of adult surgery patients, and covering a broad range of short-term and long-term surgery-related fears. The composition and phrasing of the SFQ was based on items selected from existing questionnaires $s^{4,6,10,14,23}$ and expert consultation. The selection of the initial 10 items took place after a consensus meeting of experts in the field of psychology, anaesthesiology, methodology, or epidemiology. All items are scored on an eleven point numeric rating scale (NRS) ranging from 0 (not at all afraid) to 10 (very afraid). This results in a total score of 0 to 100 . Selected items are: afraid of operation, anaesthesia, postoperative pain, side effects, health deterioration, failed operation, hospital stay, (worried) about family members, incomplete recovery, long duration of rehabilitation.

\section{Procedure}

To establish the factor structure of the SFQ, data of four different studies were used, see table 1. A two stage approach was employed. Exploratory factor analysis (EFA) was performed on the data of the first two studies, followed by confirmatory factor analysis (CFA) on the data of study 3 and 4 . EFA is used to explore the underlying factor structure of a set of items without an a priori hypothesis about the number and structure of factors to be identified. CFA is a hypothesis testing technique used to confirm the solution of the EFA in a different sample.

An initial EFA was performed on the SFQ data obtained from a prospective observational cohort study examining predictors of acute and chronic postoperative pain. ${ }^{16,17}$ The sample consisted of 1490 Dutch inpatients scheduled to undergo surgery at one of the following departments: general surgery, plastic surgery, orthopedics, ophthalmology, gynaecology, ear-nose-throat, maxillofacial surgery, urology, neurosurgery, or thoracic surgery. Table 1 presents the primary sample characteristics (Study 1). Patients completed the SFQ in the hospital one day before surgery.

To examine the robustness of the factor solution, the EFA was repeated in a second independent sample consisting of 201 women. Data were obtained from a prospective 
cohort study on predictors of acute and chronic pain after elective hysterectomy carried out in Portugal. The sample characteristics are described in table 1 (Study 2). ${ }^{18}$ In a face to face interview with a trained psychologist the SFQ was completed on the day before surgery in the hospital. For the translation of the SFQ into Portuguese a three stage procedure was performed. The first step was the forward translation of the English version into Portuguese. This was done by a bilingual person, a native speaker of the target language (Portuguese). The second step was a separate back translation. This was performed by a bilingual translator who is a native speaker of the source language (English). The translations coincided. In step three a pilot with the Portuguese version of the SFQ was performed in a sample of 46 women undergoing hysterectomy. Before surgery the SFQ was applied and participants were asked to reflect on the comprehensibility of the scale and asked for additional suggestions. The women agreed with the Portuguese translated version and showed no doubts about the items. After this the Portuguese version of the SFQ was considered ready for use.

Meanwhile a new (Brazilian) Portuguese translation was made from the original Dutch version of the scale (A.C. Mesquita, University of São Paulo at Ribeirão Preto College of Nursing). Back translation to Dutch of this version showed it to be $100 \%$ identical to the original version. This new Portuguese translation was compared to the version used in study 2 . There were only minor differences in wording which are mostly due to differences between Brazilian and European Portuguese.

On the basis of the results obtained in Study 1 and 2 two items were deleted from the SFQ and a two-factor structure was proposed for the new eight-item SFQ yielding a range of 0-80 (see below). Confirmatory factor analysis (CFA) was used to test the fit of this two-factor model against a one-factor model in a new sample of hysterectomy patients. ${ }^{24}$ Data were obtained from the first 192 included patients of an ongoing prospective multicenter study in the Netherlands on predictors of postoperative recovery after hysterectomy. Sample characteristics are presented in table 1 (Study 3). These patients completed the eight-item version of the SFQ at home in the week before surgery. 
Table 1. Sociodemographic and surgery characteristics of Study 1-5

\begin{tabular}{|c|c|c|c|c|c|}
\hline & $\begin{array}{l}\text { Study } 1 \\
\mathrm{~N}=1490\end{array}$ & $\begin{array}{l}\text { Study } 2 \\
N=201\end{array}$ & $\begin{array}{l}\text { Study } 3 \\
N=192\end{array}$ & $\begin{array}{l}\text { Study } 4 \\
N=1275\end{array}$ & $\begin{array}{l}\text { Study } 5 \\
N=75\end{array}$ \\
\hline Country & $\mathrm{NL}$ & $P$ & $N L$ & $\mathrm{NL}$ & $\mathrm{NL}$ \\
\hline Surgery & Mixed inpatient & Hysterectomy & Hysterectomy & $\begin{array}{l}\text { Mixed day } \\
\text { surgery }\end{array}$ & $\begin{array}{l}\text { Mixed day } \\
\text { surgery }\end{array}$ \\
\hline Age & $55.6 \pm 15.5$ & $51.2 \pm 9.4$ & $46.2 \pm 7.8$ & $51.9 \pm 14.7$ & $52.8 \pm 15.3$ \\
\hline \multicolumn{6}{|l|}{ Sex } \\
\hline Male & 702 & - & - & 722 & 31 \\
\hline Female & 788 & 201 & 192 & 553 & 44 \\
\hline \multicolumn{6}{|l|}{ Education } \\
\hline Low & 392 & 188 & 33 & 396 & 20 \\
\hline Intermediate \& high & 788 & 12 & 158 & 864 & 52 \\
\hline Missing & 310 & 1 & 1 & 15 & 3 \\
\hline \multicolumn{6}{|l|}{ Employment } \\
\hline Occupation & 484 & 99 & 129 & 688 & 34 \\
\hline No occupation & 684 & 102 & 60 & 586 & 37 \\
\hline Missing & 322 & 0 & 3 & 1 & 4 \\
\hline \multicolumn{6}{|l|}{ ASA } \\
\hline $1 / 11$ & 1222 & 184 & 180 & 1196 & 69 \\
\hline III / IV & 268 & 14 & 3 & 53 & 6 \\
\hline Missing & 0 & 3 & 9 & 26 & 0 \\
\hline \multicolumn{6}{|l|}{ Malignancy } \\
\hline Yes & 239 & 0 & 0 & 107 & 7 \\
\hline No & 1251 & 201 & 192 & 1168 & 68 \\
\hline Preoperative pain & $3(0-21)$ & $40(20-50)$ & $50(30-60)$ & $20(0-50)$ & $30(0-60)$ \\
\hline \multicolumn{6}{|l|}{ Expected pain } \\
\hline No / mild & 760 & 48 & 47 & 590 & 28 \\
\hline Moderate / high & 679 & 52 & 142 & 651 & 47 \\
\hline Missing / don't know & 51 & 101 & 3 & 34 & 0 \\
\hline
\end{tabular}

$\mathrm{N}$ numbers baseline population; mean \pm standard deviation, median (interquartile range).

Country: NL the Netherlands, P Portugal.

- Not applicable. Preoperative pain: VAS/NRS 0-100. Expected pain VAS/NRS 0-100 or Likert scale (Study 2): no/mild pain VAS/NRS <40, moderate/high pain VAS/NRS 40-100. ASA: American Society of Anesthesiologists. doi:10.1371/journal.pone.0100225.t001

In the last step we tested the invariance of the factor structure in a mixed male - female sample of patients undergoing various surgical procedures. Data were obtained from a prospective cohort study on the prevalence of postoperative pain in adult patients after elective day surgery performed in the Netherlands. ${ }^{25}$ The most frequently performed types of surgery in this study were, among other, general surgery, orthopaedic surgery, ear nose throat surgery, plastic surgery, and gynaecologic surgery. Sample characteristics are presented in table 1 (Study 4). A total of 1275 patients completed the eight-item version of the SFQ at home in the week before surgery. 
Convergent validity was tested by comparing the scores on the SFQ with scores on questionnaires assessing general anxiety, or negative cognitions about pain before the operation. All four studies that provided data for the psychometric evaluation of the SFQ included a measure of pain catastrophizing, either the Pain Catastrophizing Scale (PCS) or the catastrophizing subscale of the Coping Strategies Questionnaire-revised (CSQ-R). ${ }^{26,27}$ Both scales measure negative cognitions and worrying about pain. The full thirteen-item PCS was included in Study 1 and 3, a six-item abbreviated version in Study 4 and the catastrophizing subscale of CSQ-R was used in Study 2. Additionally, all studies included a pre-operative assessment of expected pain. Only Study 2 also included a measure of pre-operative general anxiety, namely the anxiety subscale of the HADS. The HADS is a widely used and well validated instrument, developed for assessing selfreported anxiety and depression. ${ }^{28}$ For the $\mathrm{PCS},{ }^{29} \mathrm{CSQ}-\mathrm{R}^{30}$ and $\mathrm{HADS}^{31}$ validity of the Portuguese and Dutch versions has been established.

Because one of the most frequently used instruments for measuring pre-operative (general) anxiety is the STAI, ${ }^{2}$ and this instrument was not included in any of the previous studies with the SFQ, we performed an additional study (Study 5) to assess convergent validity of the SFQ with the STAI. The Dutch version of the STAI has been shown to be valid. ${ }^{32}$ Both the state and trait anxiety subscales were included. In addition, patients filled out the PCS and the numerical rating scale to assess expected pain intensity. Study 5 included 75 adult patients scheduled for elective day surgery. Inclusion criteria and types of operation were similar as in study 4 . All questionnaires were completed at home in the week before surgery.

Besides construct and convergent validity, also the internal consistency of the SFQ was assessed. Therefore, in all studies Cronbach's alpha was calculated.

The next step in the validation procedure was the assessment of the sensitivity to detect differences in fear between subgroups based on age, sex, employment status, ASA (American Society of Anesthesiologists) classification, surgery because of malignancy (yes/no), preoperative pain status (no/mild or VAS/NRS $<40$, moderate/high or VAS/NRS 40-100), ${ }^{33}$ and education (lower compared to intermediate/higher education). Lower education was defined as no education, primary education, lower vocational education, or $\leq 9$ years education (Study 2). Intermediate education was defined as secondary education, intermediate vocational education, or 10-12 years of education (Study 2). Higher education was defined as higher vocational education, university, or graduation (Study 2). Finally, predictive validity was assessed on data of Study 1 and 4. Predictor variables were the SFQ and its subscales, dichotomized by median split. ${ }^{16}$ Outcome measures were acute postsurgical pain on postoperative day 4 and chronic postsurgical pain, after 6 months in Study 1 and after one year in Study 4. Another outcome measure for predictive validity was self-perceived recovery, assessed by the global surgical recovery index (GSR, range $0-100 \%$, values of $80-100 \%$ were considered as good recovery). ${ }^{16,34}$ 


\section{Statistical analysis}

Parametric data were described using mean \pm standard deviation, non-parametric data with median and interquartile range (IQR) and minimum-maximum values. EFA (principal component analyses) was performed using oblique factor rotation (oblimin). Factor extraction was based on evaluation of the scree plot and the Kaiser's criterion (factors with eigenvalues $>1$ were retained). Item selection was based on evaluation of factor loadings (cut-off value $>0.40$ ). The factor loadings can be thought of as the Pearson correlation between a factor and a variable. Item selection was further confirmed by reliability analysis (evaluation of Cronbach's alpha, values $\geq 0.7$ are considered fair and $\geq 0.8$ good). For CFA improvement of goodness of fit was assessed by Minimum Fit Function chi square. Other test criteria were the Root Mean Square Error of Approximation (RMSEA), Standardized Root Mean Square Residual (SRMR), Non-Normed Fit Index (NNFI), and the Comparative Fit Index (CFI). The RMSEA and SRMR reflect the deviation of the factor solution from the data (the lower the better, with a minimum of 0 ) and the NNFI and CFI reflect the deviation of the factor solution with the independence model (the higher the better, with a maximum around 1). Values indicating a good fit are for RMSEA $\leq 0.06$, SRMR $\leq 0.09, \mathrm{NNFI}$ and CFI $\geq 0.95 .{ }^{35}$ Convergent validity was assessed using the Pearson correlation coefficient. Sensitivity analysis was performed with the Mann Whitney U-test. For assessing predictive validity of the SFQ, odds ratios (OR) were generated by bivariate logistic regression analyses. For the descriptive statistics, EFA, reliability analysis, validity analysis, and sensitivity analysis the Statistical Package for the Social Sciences was used (SPSS version 18, Chicago, Illinois, USA). The CFA was performed with Lisrel 8.20 (Jöreskog \& Sörbom, Scientific Software International, Chicago, Illinois, USA). A $p$-value $<0.05$ was considered statistically significant for all analyses except for the convergent and predictive validity analyses. To adjust for multiple testing a Bonferroni correction of 0.05:3 was applied resulting in a $p$-value $<0.017$ considered statistically significant for all Pearson correlation coefficients and logistic regression analyses.

\section{RESULTS}

\section{Study 1. Exploratory factor analysis: initial results}

The original 10 -item version of the SFQ was completed by 1490 patients. A median score of 23 was obtained (IQR 11-38). In table 2 median and minimum-maximum scores are presented. The scores of all items comprised the whole range from 0-10 indicating an appropriate item scaling, although some floor effect cannot be excluded since the distribution is skewed to the right. 
Table 2. SFQ scores of Study 1-5

\begin{tabular}{lllllll}
\hline & $\begin{array}{l}\text { Study } 1 \\
10 \text { items }\end{array}$ & $\begin{array}{l}\text { Study 2 } \\
10 \text { items }\end{array}$ & $\begin{array}{l}\text { Study 2 } \\
8 \text { items }\end{array}$ & $\begin{array}{l}\text { Study } 3 \\
8 \text { items }\end{array}$ & $\begin{array}{l}\text { Study } 4 \\
\text { items }\end{array}$ & 8 items \\
\hline SFQ & $23(0-98)$ & $20(0-82)$ & $13(0-62)$ & $22.9(0-77)$ & $22(0-80)$ & $25(0-66)$ \\
SFQ-S & $12(0-40)$ & $9(0-36)$ & $9(0-36)$ & $14(0-40)$ & $14(0-40)$ & $14(0-38)$ \\
SFQ-I & $9.5(0-60)$ & $10(0-48)$ & $3(0-35)$ & $7(0-38)$ & $8(0-40)$ & $9(0-32)$ \\
\hline
\end{tabular}

Median (minimum-maximum).

SFQ-S: Surgical Fear Questionnaire short-time consequences (item 1-4), SFQ-I: SFQ long-term consequences (10-item version: item 5-10; 8-item version: item 5, 6, 9,10). doi:10.1371/journal.pone.0100225.t002

The EFA identified two factors together explaining $60.2 \%$ of the total variance. All items loaded adequately (defined as $>0.40$ ) on one of the two factors (see table 3 ). Inspection of the items indicated that the items of one of the factors referred to more proximal fears (item 1-4; e.g. fear of pain, fear of anaesthesia) while the items in the other factor referred to more distal fears (item 5-10; e.g. fear of incomplete recovery, fear of long rehabilitation). These factors were labelled "fear of the short-term consequences of surgery" (SFQ-S) and "fear of the long-term consequences of surgery" (SFQ-I) respectively. Cronbach's alpha of the SFQ-s was 0.83, of SFQ-I 0.82 and of the total scale 0.87. The intercorrelation between SFQ-I and SFQ-S was 0.57, $p<0.01$.

This initial EFA thus indicated a two-factor model for the SFQ comprising all ten items. To examine the robustness of this factor structure and the generalizability to a different population, the EFA was repeated using data from a Portuguese study on women undergoing hysterectomy.

\section{Study 2. Exploratory factor analysis: confirmation in an independent sample}

The 10-item SFQ was completed by 201 patients. Compared to our previous sample, patients in this sample scored somewhat lower on most items with a median score of 20 (IQR 10-32), see also table 2. All ten items yielded scores ranging the full scale from 0-10. The distribution was skewed to the right.

EFA again revealed a two-factor structure similar to Study 1, explaining 50.3\% of the variance. One factor contained items related to fear of short-term consequences of surgery (item 1-4) and one factor contained items related to long-term consequences of surgery (item 5, 6, 9, 10). However, two items (item 7: "I am afraid of staying in the hospital" and item 8 "I worry about my family") did not load above the cut-off of $>0.40$ on either of the two factors (table 3). These were also the two items that had the lowest factor loading in the previous sample, with loadings well below the other items on the same factor. Moreover, Cronbach's alpha on the SFQ-I subscale indicated only moderate internal consistency (0.63). Deleting these two insufficiently loading items increased the Cronbach's alpha of the SFQ-I subscale to 0.77. Cronbach's alpha of the total scale increased from 0.77 to 0.80 ; Cronbach's alpha for SFQ-s was 0.77. Intercorrelation be- 
tween the SFQ-S and SFQ-I subscale was $0.41, p<0.01$. A post hoc reliability analysis on the SFQ eight-item total scale and four-item SFQ-I subscale of Study 1 revealed a Cronbach's alpha of 0.87 on the total scale (unchanged) and of 0.84 on the SFQ-I.

Table 3. Exploratory factor analysis

\begin{tabular}{|c|c|c|c|c|c|}
\hline & & \multicolumn{2}{|l|}{ Study 1} & \multicolumn{2}{|l|}{ Study 2} \\
\hline & & SFQ-S & SFQ-I & SFQ-S & SFQ-I \\
\hline & Eigenvalue & 1.211 & 4.807 & 3.588 & 1.440 \\
\hline 1 & Operation & 0.845 & 0.035 & 0.889 & 0.091 \\
\hline 2 & Anaesthesia & 0.907 & -0.123 & 0.756 & 0.045 \\
\hline 3 & Pain & 0.657 & 0.200 & 0.695 & -0.073 \\
\hline 4 & Side effects & 0.740 & 0.054 & 0.719 & 0.034 \\
\hline 5 & Health deterioration & 0.040 & 0.768 & 0.066 & -0.728 \\
\hline 6 & Failed operation & 0.013 & 0.776 & -0.068 & -0.761 \\
\hline 7 & Hospital stay & 0.256 & 0.434 & 0.393 & -0.125 \\
\hline 8 & Family & 0.156 & 0.464 & 0.316 & -0.008 \\
\hline 9 & Incomplete recovery & -0.114 & 0.931 & 0.094 & -0.805 \\
\hline 10 & Long rehabilitation & -0.057 & 0.834 & -0.028 & -0.770 \\
\hline
\end{tabular}

Eigenvalues and factor loadings.

doi:10.1371/journal.pone.0100225.t003

Based on the factor loadings and internal consistency, the SFQ can best be used as an eight-item scale with two subscales, each consisting of four items. In the next step, we performed a confirmatory factor analysis on the eight-item SFQ in a new sample of women undergoing hysterectomy. We compared the two-factor model with a onefactor model. It may be argued that a one-factor model is equally suitable and more parsimonious for the data because of the high internal consistency of the total scale and the moderate but significant intercorrelation between the subscales.

\section{Study 3. Confirmatory factor analysis}

A total of 192 women scheduled for hysterectomy completed the SFQ pre-operatively. Median fear response of this sample was higher than the two previous samples with a score of 22.9 (IQR 11-37) on the eight-item version of the SFQ, which is as high as the score on the ten-item version in our initial sample and even higher than the scores of the Portuguese women on the ten-item version (table 2). Similar as in the previous samples the distribution was skewed to the right, and all item scores covered the full range of $0-10$.

CFA was performed to compare a one-factor model with the two-factor model as determined by the previous EFA. Table 4 displays the results of the CFA. All test criteria indicated a poor fit of the one-factor model. The two-factor model revealed a fair model 
fit, except for the RMSEA. Cronbach's alpha was 0.89 for the total scale and 0.86 and 0.87 for the SFQ-S and SFQ-I respectively. Intercorrelation between the SFQ-S and SFQ-I subscale was $0.61, p<0.01$.

Thus, based on factor analyses in the first three studies, the two-factor model seems most appropriate for the SFQ. However, the second EFA and the CFA were both performed in an entirely female sample undergoing hysterectomy in an inpatient setting. To exclude that these results are population specific, we repeated the CFA in male and female patients undergoing various procedures in day surgery setting. It may be expected that these procedures are more minor and possibly elicit less fear. Because the SFQ is meant to be generally applicable in all kind of surgical settings, generalizability of the results to another setting is important.

\section{Study 4. Confirmatory factor analysis: generalization to day surgery patients}

The eight-item SFQ was completed by 1275 patients at home in the week before surgery. In contrast to our expectation, day surgery patients scored equally high on the SFQ as inpatients, with a median score of 22 (IQR 11-36). This was also true for the subscale fear of long-term consequences, see table 2. All item scores covered the full range of 0-10. Similar to the results in the inpatient sample, the one-factor model did not show adequate fit, whereas the parameters of the two-factor model indicated a fair model, except for the RMSEA. Cronbach's alpha was excellent, i.e. 0.91 for the total scale and 0.88 and 0.89 for the SFQ-S and SFQ-I respectively. Intercorrelation between the SFQ-S and SFQ-I subscale was $0.65, p<0.01$.

Table 4. Confirmatory factor analysis

\begin{tabular}{llllll}
\hline & \multicolumn{3}{l}{ Study 3} & & \multicolumn{3}{l}{ Study 4} & \\
\cline { 2 - 3 } \cline { 5 - 6 } & 1 Factor & 2 Factors & & 1 Factor & 2 Factors \\
\hline Minimum Fit Function chi square $(d f)$ & $251.9179(20)$ & $88.6924(19)$ & & $1212.4356(20)$ & $346.5056(19)$ \\
RMSEA & 0.2730 & 0.1357 & & 0.2495 & 0.1206 \\
Standardized RMR & 0.1010 & 0.0586 & & 0.0838 & 0.0419 \\
NNFI & 0.6435 & 0.8872 & & 0.7356 & 0.9236 \\
CFI & 0.7476 & 0.9235 & 0.8112 & 0.9481 \\
\hline
\end{tabular}

Minimum Fit Function chi square: improvement of 2 factor model compared to 1 factor model 163.2255 ( $d f$ 1), $p<0.0001$ (Study 3) and 865.93 (df1) $p<0.0001$ (Study 4).

Df: degrees of freedom. RMSEA: Root Mean Square Error of Approximation, Standardized RMR: Standardized Root Mean Square Residual, NNFI: Non-Normed Fit Index, CFI: Comparative Fit Index.

doi:10.1371/journal.pone.0100225.t004

In sum, the SFQ can best be conceived as an eight-item questionnaire consisting of two subscales, with four items measuring fear of the short-term consequences of surgery and four items measuring fear of long-term consequences. The factor structure appears to be robust across different populations and in different languages (Dutch vs. Portu- 
guese). In the next step we assessed the convergent validity of the SFQ with other instruments that have been used to measure pre-operative anxiety or worries, i.e. the PCS, the HADS and the STAI. Also we correlated the SFQ score with pre-operatively assessed expected pain after surgery.

\section{Study 1-5. Convergent validation}

Data were obtained from the four studies presented above and from Study 5, which was specifically set-up to further examine convergent validity. Median score on the SFQ in this latter study was 25 (IQR 10-39.3), see table 2. Cronbach's alpha was again excellent with 0.92 for the SFQ, 0.88 for SFQ-s and 0.90 for SFQ- I. Intercorrelation between the SFQ-S and SFQ-I subscale was 0.73, $p<0.01$.

Table 5. Correlations of the SFQ with pain catastrophizing, expected pain, and state anxiety

\begin{tabular}{|c|c|c|c|c|c|c|c|}
\hline & & & Study 1 & Study 2 & Study 3 & Study 4 & Study 5 \\
\hline \multirow[t]{3}{*}{ Pain Catastrophizing } & / & SFQ & $0.41^{1}$ & $0.44^{2}$ & $0.32^{1}$ & $0.45^{1}$ & $0.60^{1}$ \\
\hline & & SFQ-S & 0.34 & 0.36 & 0.28 & 0.41 & 0.47 \\
\hline & & SFQ-I & 0.40 & 0.41 & 0.31 & 0.42 & 0.66 \\
\hline \multirow[t]{3}{*}{ Expected Pain } & / & SFQ & 0.33 & - & 0.39 & 0.48 & 0.45 \\
\hline & & SFQ-S & 0.33 & - & 0.42 & 0.46 & 0.40 \\
\hline & & SFQ-I & 0.26 & - & 0.27 & 0.42 & 0.42 \\
\hline \multirow[t]{3}{*}{ State anxiety } & / & SFQ & - & $0.56^{3}$ & - & - & $0.70^{4}$ \\
\hline & & SFQ-S & - & 0.53 & - & - & 0.62 \\
\hline & & SFQ-I & - & 0.40 & - & - & 0.66 \\
\hline
\end{tabular}

Pearson correlation, all significant at 0.01 level. - Not applicable.

SFQ: eight items; SFQ-s: Surgical Fear Questionnaire short-time consequences (item 1-4), SFQ-I: SFQ longterm consequences (item 5, 6, 9, and 10).

Catastrophizing: ${ }^{1}$ PCS: Pain Catastrophizing Scale, (Study 1, 3 and 513 items; Study 4 six items: I feel I can't stand it any more, I become afraid that the pain may get worse, I can't seem to keep it out of my mind, I keep thinking about how badly I want the pain to stop, there is nothing I can do to reduce the intensity of the pain, I wonder whether something serious may happen). Catastrophizing: ${ }^{2} \mathrm{CSQ}$-c: Coping Strategies QuestionnaireRevised, subscale pain catastrophizing.

State anxiety: ${ }^{3}$ HADS-a: Hospital Anxiety and Depression Scale, anxiety subscale. State anxiety: ${ }^{4}$ STAl: StateTrait Anxiety Inventory, state subscale. doi:10.1371/journal.pone.0100225.t005

Table 5 shows the Pearson correlation coefficients between the SFQ and its subscales with pain catastrophizing, expected pain, and general anxiety for all five studies. To facilitate comparison of the results across the five studies, correlation coefficients were calculated using the SFQ eight item version. Correlations between the SFQ and the three other scales were significant at 0.01 level. The correlation with pain catastrophizing ranged from 0.32 to 0.60 and with expected pain from 0.33 to 0.48 . For the two studies assessing state anxiety (HADS or STAI-state anxiety subscale) correlations with SFQ were 0.56 and 0.70 respectively. In most cases the correlations with the SFQ total 
score were slightly higher compared to the correlations with the SFQ-S and SFQ-I. In Study 5 also the STAI-trait anxiety subscale was assessed. The correlation between the SFQ and trait anxiety was significant, but the values of 0.45 for the SFQ, 0.40 for the SFQ-S, and 0.42 for the SFQ-I were lower compared to state anxiety.

Thus, the SFQ appeared to be significantly related to other instruments used to assess pre-operative anxiety or worry, in particular to the HADS and the STAI-state anxiety subscale. In the next step we looked at the sensitivity of the SFQ to detect the hypothesized differences in fear in certain subgroups. In accordance with previous studies, we expected that female patients, younger patients, and patients with less education would score higher on surgical fear. The other factors were included exploratory.

\section{Study 1-5. Sensitivity to differences in patient characteristics}

To assess the effect of different patient characteristics on the SFQ the following subgroups were defined: age $<65$ years compared to $\geq 65$ years (in Study 3 patients older than 65 years were excluded), males compared to females, lower compared to intermediate/higher education, employed compared to not employed, ASA classification I/II compared to ASA III/IV, malignancy as indication for surgery yes/no, and preoperative pain $<40$ compared to $\geq 40$ on $100 \mathrm{~mm}$ VAS. In table 6 the results of the sensitivity analyses are presented, again using the SFQ eight-item score across all five studies. In general, the results for the SFQ-S and SFQ-I subscales were in line with the SFQ total score. As expected, SFQ scores of the younger participants were higher compared to those reported by older participants although only in the Portuguese sample this difference reached statistical significance. Also in line with our expectations, females appeared to be more fearful about the surgery compared to males (Study 1 and 4 significant, Study 5 non significant (ns)). Concerning the effect of education on surgical fear, a difference between the Portuguese and the Dutch populations occurred: in the Portuguese population lower education level was associated with a lower level of surgical fear (ns) whereas in all four Dutch populations lower educated participants scored higher compared to intermediate or higher educated participants. In two out of five studies participants with an occupation scored significantly higher on the SFQ compared to participants without an occupation. ASA-classification did not affect SFQ scores. SFQ results for the subgroups concerning malignancy or not as indication for surgery revealed no differences in the studies 1 and 4 with large population samples. In the smaller Study 5 malignancy did lead to significantly increased surgical fear. Finally, preoperative pain was associated with increased surgical fear across all five studies. 


\section{Chapter 3}

Table 6. Sensitivity to differences in patient characteristics

\begin{tabular}{|c|c|c|c|c|c|}
\hline & Study 1 & Study 2 & Study 3 & Study 4 & Study 5 \\
\hline \multicolumn{6}{|l|}{ Age } \\
\hline$<65$ & $20(9-33)$ & $14(6-25)^{* *}$ & $22.9(11-37)$ & $23(12-37)$ & $25(11-40)$ \\
\hline$\geq 65$ & $19(8-33)$ & $6(0-15)$ & NA & $21(8-35)$ & $22(6-35)$ \\
\hline \multicolumn{6}{|l|}{ Sex } \\
\hline Male & $15(6-27)^{* * *}$ & NA & NA & $19(8-31)^{* * *}$ & ${ }^{\mathrm{a}} 17(9-34)$ \\
\hline Female & $24.5(12.9-36.7)$ & $13(5-24)$ & $22.9(11-37)$ & $26(13.3-40)$ & $26(12-44)$ \\
\hline \multicolumn{6}{|l|}{ Education } \\
\hline Low & $22(8.8-34.1)$ & ${ }^{\mathrm{a}} 12(4.3-23.8)$ & $25(13.5-41)$ & b25 (11.3-39)* & $28(6-44)$ \\
\hline Intermediate \& hig & 19 (9-31) & $19.5(11.8-24.5)$ & $21(10-36)$ & $22(11-35.7)$ & $25(10.3-38.8)$ \\
\hline \multicolumn{6}{|l|}{ Employment } \\
\hline Occupation & ${ }^{\mathrm{a}} 21(10-34)^{* *}$ & ${ }^{c} 14(6-28)^{*}$ & $24(11.3-36)$ & ${ }^{b} 22(11-36)$ & $25(10.8-38.3)$ \\
\hline No occupation & $18(8-30)$ & $11.5(2.8-22)$ & $20(8-38)$ & $23(11-38)$ & $22.5(6.5-39.3)$ \\
\hline \multicolumn{6}{|l|}{ ASA } \\
\hline $1 / 11$ & $20(9-33)$ & $14(5-24)$ & $24(11-37)$ & $22(11-36)$ & ${ }^{\mathrm{a}} 25(11.3-39.8)$ \\
\hline III / IV & $20.6(9.5-33.6)$ & $12(8-23)$ & $10(4-N C)$ & $25(12.5-36)$ & $9.5(3-25)$ \\
\hline \multicolumn{6}{|l|}{ Malignancy } \\
\hline Yes & $20(9-33)$ & NA & NA & $24.5(13-40)$ & ${ }^{a} 36(25-58)^{*}$ \\
\hline No & $20(9-33)$ & & & $22(11-36)$ & $23(10-38)$ \\
\hline \multicolumn{6}{|l|}{ Preoperative pain } \\
\hline No / mild & $19(8-32)^{* * *}$ & $14(6.5-22)$ & $18.5(7.8-32.3)$ & $18(9-31)^{* * *}$ & $17(6-31)^{* *}$ \\
\hline Moderate / high & $26.1(13.5-39)$ & $15(5-25)$ & $26(14-40)$ & $29(16-42)$ & $35(14-43)$ \\
\hline
\end{tabular}

SFQ (eight items), median (interquartile range). ${ }^{*} p<0.05, * * p<0.01, * * * p<0.001$. NA not applicable: hysterectomy patients only, malignancy excluded; in Study 3 age $\geq 65$ excluded. NC not calculable. ASA: American Society of Anesthesiologists.

Deviation of SFQ-short term and SFQ-long term subscale from the SFQ results is indicated as: ${ }^{a}$ SFQ-s significant difference and SFQ-I non significant difference; ${ }^{b}$ SFQ-S non significant difference and SFQ-I significant difference; 'SFQ-S and SFQ-I non significant difference.

Preoperative pain: no/mild or VAS/NRS <40, moderate/high or VAS/NRS 40-100, Study 1: pain at time of completion questionnaire, Study 2-5: average pain last week.

doi:10.1371/journal.pone.0100225.t006

The final part of this paper presents data on the predictive validity of the SFQ for acute and chronic post-operative pain and for perceived recovery. We also compare the predictive value of the total SFQ score with that of its two subscales.

\section{Study 1 and 4. Predictive validity}

Median split was used to identify fearful and non-fearful patients. Using OR's generated by bivariate logistic regression analyses, the predictive value of the SFQ for pain and recovery was assessed. Pain scores were dichotomized using a cut-off value of 40 for the VAS/NRS. Values of $80-100 \%$ were considered as (near) optimal recovery, on the 
GSR scale of $0-100 \%$. Results are presented in table 7. Acute pain as well as long-term pain was more strongly predicted by the scores on the SFQ-I subscale than the scores on the SFQ-S subscale. Also for recovery the SFQ-I was the strongest predictor. The predictive value of the SFQ total score was in most cases only slightly lower than that of the SFQ-I score. Predictive value of the two subscales of the SFQ for post-operative pain and perceived recovery using multivariate logistic regression analyses was previously reported in studies of Gramke et al. ${ }^{36}$ and Peters et al. ${ }^{17}$

Table 7. Predictive validity of the SFQ, SFQ-S and SFQ-I

\begin{tabular}{llllll}
\hline & & \multicolumn{2}{l}{ Study 1} & \multicolumn{2}{l}{ Study 4} \\
Outcome & Predictor & \multicolumn{2}{l}{ OR $(95 \mathrm{Cl})$} & \multicolumn{2}{l}{ OR (95 Cl) } \\
\hline APSP & SFQ & 2.73 & $(1.59-4.69)^{* * *}$ & 2.35 & $(1.81-3.04)^{* * *}$ \\
APSP & SFQ-s & 1.83 & $(1.08-3.10)$ & 2.12 & $(1.64-2.73)^{* * *}$ \\
APSP & SFQ-I & 3.55 & $(1.99-6.32)^{* * *}$ & 2.62 & $(2.02-3.39)^{* * *}$ \\
CPSP & SFQ & 1.77 & $(1.25-2.51)^{* *}$ & 2.28 & $(1.56-3.34)^{* * *}$ \\
CPSP & SFQ-S & 1.66 & $(1.16-2.37)^{* *}$ & 1.66 & $(1.15-2.39)^{* *}$ \\
CPSP & SFQ-I & 1.77 & $(1.24-2.51)^{* *}$ & 3.05 & $(2.06-4.51)^{* * *}$ \\
GSR & SFQ & 0.44 & $(0.31-0.62)^{* * *}$ & 0.56 & $(0.41-0.77)^{* * *}$ \\
GSR & SFQ-S & 0.61 & $(0.43-0.87)^{* *}$ & 0.77 & $(0.56-1.05)$ \\
GSR & SFQ-I & 0.44 & $(0.31-0.63)^{* * *}$ & 0.40 & $(0.29-0.55)^{* * *}$ \\
\hline
\end{tabular}

Bivariate logistic regression with median split SFQ, SFQ short term and SFQ long term as predictor. APSP: acute postsurgical pain on day 4. CPSP: chronic postsurgical pain after 6 months in Study 1, after one year in Study 4. Pain scores were dichotomized using a cut-off value of 40 for the VAS/NRS. GSR: global surgical recovery on a scale of 0-100\%, values of $80-100 \%$ were considered as good recovery; long term GSR after 6 months in Study 1, after one year in Study 4.

To adjust for multiple testing a Bonferroni correction was applied: a $p$-value $<0.017$ was considered statistically significant. ${ }^{* *} p<0.01,{ }^{* * *} p<0.001$.

doi:10.1371/journal.pone.0100225.t007

\section{DISCUSSION}

The aim of the present study was to establish the reliability and validity of the SFQ. Therefore use was made of data obtained from 5 prospective studies ( $N=3233$ ) in inpatient or day surgery patients. Exploratory and confirmatory factor analyses indicated that a two-factor model best describes the structure of the SFQ. Two four-item subscales can be distinguished: fear of immediate consequences of surgery and fear of the long-term consequences. However, the high internal consistency of the SFQ (eight-item total score) and the moderate, but significant intercorrelations between the SFQ shortterm and long-term subscales indicate that the SFQ total score may also be suitable for use in studies on surgical fear. This is further attested by the almost comparable predictive value of the SFQ total score compared to the SFQ-I subscale, and both being stronger related to the patient-reported outcomes than the SFQ-s subscale. 
Significant intercorrelations with other validated instruments for the measurement of preoperative fear such as pain catastrophizing, expected postoperative pain and state anxiety indicate good convergent validation of the SFQ. As we expected, the SFQ can be used in day surgery as well as in inpatient surgery and has an adequate sensitivity for differences with regard to sex and age, and in the Dutch samples also for education level.

A limitation of this paper is that the SFQ in all five studies was assessed once in the week or evening before surgery. There are no data yet on the effect of preoperative time course on SFQ scores. For coronary surgery patients, Koivula ${ }^{5}$ assessed surgical fear during the waiting period at home, at hospital admission, and after surgery. Preoperative fear and anxiety levels were highest during the waiting period at home and dropped after hospital admission. But for other types of surgery most studies only measure preoperative fear or anxiety in the week before surgery. ${ }^{37-41}$ However, in the case of undesirable high levels of preoperative fear, treatment will be advocated. Depending on the type of intervention, a certain amount of time may be needed before the intended reduction of surgical fear can be achieved. Therefore, to enable preoperative treatment of surgical fear, as well as to further explore the optimal time point for the assessment of surgical fear, a study measuring the SFQ at different time points, starting from preoperative screening until the day of surgery is necessary. Secondly, the differences in SFQ scores between the Portuguese population and the Dutch population with regard to age, sex, education, and employment status raise the question to what extent sociodemographic factors affect the SFQ. Therefore, the stability of the SFQ across different subgroups needs further exploration. Thirdly, because of the non parametric distribution of the SFQ a median split was used for predictive logistic regression analyses. However, for practical use, e.g. selection of the most fearful patients for preoperative treatment of surgical fear, a more stringent cut-off point seems indicated.

Implications for practice. This paper demonstrated that the SFQ is a concise and generic instrument for the assessment of surgical fear, suitable for most types of elective adult surgery. For further research we suggest additional testing of the convergent validation using biomarkers such as preoperative stress hormone levels. Also the effect of linguistic and cultural influences on the SFQ needs further study. Finally, for diagnostic use optimal cut-off points of the SFQ need to be established. We conclude that the SFQ is a valid and reliable eight-item index of surgical fear, consisting of two subscales: fear of the short-term consequences of surgery and fear of the long-term consequences.

\section{ACKNOWLEDGEMENTS}

We thank Nieke Oversier for the effective patient recruitment and data entry for Study 5 during her scientific traineeship at our department. 


\section{REFERENCES}

1. Munafo MR, Stevenson J Anxiety and surgical recovery. Reinterpreting the literature. J Psychosom Res. 2001; 51(4): 589-596.

2. Theunissen M, Peters ML, Bruce J, et al. Preoperative anxiety and catastrophizing: a systematic review and meta-analysis of the association with chronic postsurgical pain. Clin J Pain. 2012; 28(9): 819-841.

3. Zieger $\mathrm{M}$, Schwarz $\mathrm{R}$, Konig $\mathrm{HH}$, et al. Depression and anxiety in patients undergoing herniated disc surgery: relevant but underresearched - a systematic review. Cent Eur Neurosurg. 2010; 71(1): 26-34.

4. Graham LE, Conley EM Evaluation of anxiety and fear in adult surgical patients. Nurs Res. 1971; 20(2): 113-122.

5. Koivula M, Tarkka MT, Tarkka M, et al. Fear and anxiety in patients at different time-points in the coronary artery bypass process. Int J Nurs Stud. 2002; 39(8): 811-822.

6. Shafer A, Fish MP, Gregg KM, et al. Preoperative anxiety and fear: a comparison of assessments by patients and anesthesia and surgery residents. Anesth Analg. 1996; 83(6): 1285-1291.

7. Carr E, Brockbank K, Allen S, et al. Patterns and frequency of anxiety in women undergoing gynaecological surgery. J Clin Nurs. 2006; 15(3): 341-352.

8. Caumo W, Schmidt AP, Schneider CN, et al. Risk factors for preoperative anxiety in adults. Acta Anaesthesiol Scand. 2001; 45(3): 298-307.

9. Millar K, Jelicic M, Bonke B, et al. Assessment of preoperative anxiety: comparison of measures in patients awaiting surgery for breast cancer. Br J Anaesth. 1995; 74(2): 180-183.

10. Koivula M, Tarkka MT, Tarkka M, et al. Fear and in-hospital social support for coronary artery bypass grafting patients on the day before surgery. Int J Nurs Stud. 2002; 39(4): 415-427.

11. Rosenberger PH, Kerns R, Jokl P, et al. Mood and attitude predict pain outcomes following arthroscopic knee surgery. Ann Behav Med. 2009; 37(1): 70-76.

12. Zigmond AS, Snaith RP The hospital anxiety and depression scale. Acta Psychiatr Scand. 1983; 67(6): 361370.

13. Spielberger CD Assessment of state and trait anxiety: conceptual and methodological issues. South Psychol. 1985; 2: 6-16.

14. Boker A, Brownell L, Donen N The Amsterdam preoperative anxiety and information scale provides a simple and reliable measure of preoperative anxiety. Can J Anaesth. 2002; 49(8): 792-798.

15. Moerman N, van Dam FS, Muller MJ, et al. The Amsterdam Preoperative Anxiety and Information Scale (APAIS). Anesth Analg. 1996; 82(3): 445-451.

16. Peters ML, Sommer M, de Rijke JM, et al. Somatic and psychologic predictors of long-term unfavorable outcome after surgical intervention. Ann Surg. 2007; 245(3): 487-494.

17. Peters $\mathrm{ML}$, Sommer $\mathrm{M}$, van Kleef $\mathrm{M}$, et al. Predictors of physical and emotional recovery 6 and 12 months after surgery. Br J Surg. 2010; 97(10): 1518-1527.

18. Pinto $P R, M c I n t y r e ~ T$, Almeida $A$, et al. The mediating role of pain catastrophizing in the relationship between presurgical anxiety and acute postsurgical pain after hysterectomy. Pain. 2012; 153(1): 218226.

19. Pinto PR, Mclntyre T, Fonseca C, et al. Pre- and post-surgical factors that predict the provision of rescue analgesia following hysterectomy. Eur J Pain. 2012.

20. Pinto PR, Mclntyre T, Nogueira-Silva C, et al. Risk factors for persistent postsurgical pain in women undergoing hysterectomy due to benign causes: a prospective predictive study. J Pain. 2012; 13(11): 1045-1057.

21. Sommer M, de Rijke JM, van Kleef $M$, et al. Predictors of acute postoperative pain after elective surgery. Clin J Pain. 2010; 26(2): 87-94

22. Sommer M, Geurts JW, Stessel B, et al. Prevalence and predictors of postoperative pain after ear, nose, and throat surgery. Arch Otolaryngol Head Neck Surg. 2009; 135(2): 124-130.

23. Johnston M Recognition of patients' worries by nurses and by other patients. Br J Clin Psychol. $1982 ; 21$ (Pt 4): 255-261. 


\section{Chapter 3}

24. Peters ML, Theunissen HMS, Maas J, et al. Recovery after hysterectomy: A study into influencing factors (study protocol). 2011; Available from: http://www.trialregister.nl/trialreg/admin/rctview.asp?TC=2702.

25. Fiddelers A, Hoofwijk D, Gramke H-F, et al., How does persistent postoperative pain correlate with preoperative pain and acute postoperative pain? (Abstract), in Eur J Anaesthesiol 2012. p. 198.

26. Riley JL, Robinson ME CSQ: five factors or fiction? Clin J Pain. 1997; 13(2): 156-162.

27. Sullivan MJL, Bishop SR, Pivik J The Pain Catastrophizing Scale: Development and validation. Psychological Assessment. 1995; 7(4): 524-532.

28. Bjelland I, Dahl AA, Haug TT, et al. The validity of the Hospital Anxiety and Depression Scale. An updated literature review. J Psychosom Res. 2002; 52(2): 69-77.

29. Van Damme S, Crombez G, Bijttebier P, et al. A confirmatory factor analysis of the Pain Catastrophizing Scale: invariant factor structure across clinical and non-clinical populations. Pain. 2002; 96(3): 319-324.

30. Ferreira-Valente MA, Ribeiro JL, Jensen MP, et al. Coping with chronic musculoskeletal pain in Portugal and in the United States: a cross-cultural study. Pain Med. 2011; 12(10): 1470-1480.

31. Pais-Ribeiro J, Silva I, Ferreira T, et al. Validation study of a Portuguese version of the Hospital Anxiety and Depression Scale. Psychol Health Med. 2007; 12(2): 225-235; quiz 235-227.

32. Van der Ploeg HM, Defares PB, Spielberger CD, Handleiding bij de Zelf-Beoordelings Vragenlijst ZBV: Een Nederlandstalige bewerking van de Spielberger State-Trait Anxiety Inventory STAI-DY [Manual for the Self-Judgment Questionnaire ZBV: A Dutch adaptation of the Spielberger State-Trait Anxiety Inventory STAI-DY]. 1980, Swets \& Zeitlinger: Lisse.

33. Dihle A, Helseth S, Paul SM, et al. The exploration of the establishment of cutpoints to categorize the severity of acute postoperative pain. Clin J Pain. 2006; 22(7): 617-624.

34. Kleinbeck SV Self-reported at-home postoperative recovery. Res Nurs Health. 2000; 23(6): 461-472.

35. Hu L, Bentler PM Cutoff criteria for fit indexes in covariance structure analyses: Conventional criteria versus new alternatives. Structural Equation Modeling. 1999; 6(1): 1-55.

36. Gramke HF, de Rijke JM, van Kleef $M$, et al. Predictive factors of postoperative pain after day-case surgery. Clin J Pain. 2009; 25(6): 455-460

37. Johnston M Anxiety in surgical patients. Psychol Med. 1980; 10(1): 145-152.

38. de Groot KI, Boeke S, van den Berge HJ, et al. Assessing short- and long-term recovery from lumbar surgery with pre-operative biographical, medical and psychological variables. Brit J Health Psychol. 1997; 2: 229-243.

39. Ene KW, Nordberg G, Johansson FG, et al. Pain, psychological distress and health-related quality of life at baseline and 3 months after radical prostatectomy. BMC Nurs. 2006; 5: 8.

40. Graver V, Ljunggren AE, Malt UF, et al. Can psychological traits predict the outcome of lumbar disc surgery when anamnestic and physiological risk factors are controlled for? Results of a prospective cohort study. J Psychosom Res. 1995; 39(4): 465-476.

41. Poleshuck EL, Katz J, Andrus CH, et al. Risk Factors for Chronic Pain Following Breast Cancer Surgery: A Prospective Study. J Pain. 2006; 7(9): 626-634. 


\section{SUPPORTING INFORMATION}

\section{S1 Surgical Fear Questionnaire}

Patient instructions:

This questionnaire assesses how afraid you are for various aspects related to the surgical procedure you are about to undergo. Please circle the number that best reflects how you feel right now.

1. I am afraid of the operation

2. I am afraid of the anaesthesia

3. I am afraid of the pain after the operation

4. I am afraid of the unpleasant side effects (like nausea) after the operation

5. I am afraid my health will deteriorate because of the operation

6. I am afraid the operation will fail

7. (I am afraid of staying in the hospital)*

8. (I worry about my family)*

9. I am afraid that I won't recover completely from the operation

10. I am afraid of the long duration of the rehabilitation after the operation

*Note: in the final version item seven and eight are left out.

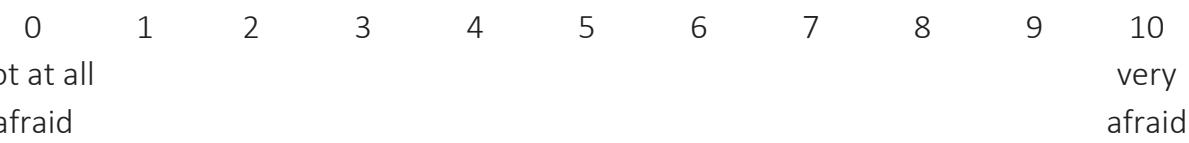

For the calculation of the total score the following instructions are applicable: one missing item score at maximum is allowed, to be replaced by the subject's mean score. In the case of more than one missing, the SFQ should not be interpreted. For the calculation of the subscales (short-term fear item 1-4 and long-term fear item 5, 6, 9, 10) no missings are allowed. If a subject enters two scores for one item: if adjacent, choose the highest value, if non-adjacent (other values are in between) the item has to be considered as missing. 



\section{Chapter}

\section{Validity and time course of surgical fear as measured with the Surgical Fear Questionnaire in patients undergoing cataract surgery}

This chapter is submitted for publication as: Maurice Theunissen, Soraya Jonker, Jan Schepers, Nancy A. Nicolson, Rudy Nuijts, Hans-Fritz Gramke, Marco A.E. Marcus, MadeIon L. Peters. Validity and time course of surgical fear as measured with the Surgical Fear Questionnaire in patients undergoing cataract surgery. 


\section{ABSTRACT}

Objectives: The primary aim of the study was to assess the convergent validity of the Surgical Fear Questionnaire (SFQ) with other self-report instruments and biological indices of stress. Secondary aims were the examination of predictors of the level and time course of fear and preferences for fear treatment.

Methods: In a prospective observational cohort study SFQ short-term (SFQ-s) and longterm (SFQ-I) scores were assessed one week, one day, and the morning before cataract surgery, together with salivary cortisol and alpha-amylase (SAA) levels, and numeric rating scale (NRS) fear score. SFQ-scores were also assessed before second eye surgery. Expected pain and recovery, and sociodemographic and medico-psychological predictors of fear were assessed at baseline.

Results: Data of 98 patients were analyzed. Scores of both SFQ-subscales (range 0-40) were generally low, all mean $\leq 9.0$. SFQ-S and SFQ-I correlated significantly with the other self-report instruments: NRS fear .83 and .89 , expected pain .49 and .54 , expected recovery -.27 and -.44 . No association was found between SFQ-scores and cortisol or sAA level. Predictors of the level of fear were baseline pain and stress. Additional effects of time were found for subgroups based on educational level, antidepressant use, and presurgical stress (SFQ-I). SFQ-scores were significantly lower before the second cataract surgery than before the first, and higher in patients who would have appreciated treatment of fear.

Discussion: Convergent validity of the SFQ with other self-report measures is shown. The sensitivity of the SFQ permits the detection of small variations in fear caused by time or other factors. 


\section{INTRODUCTION}

Across several studies surgical fear has been associated with increased postoperative pain, increased use of analgesia, and poor postoperative recovery, physical functioning, or mental health. ${ }^{1-6}$ As a result, assessment of surgical fear seems important because it is the first step towards preoperative treatment of fearful patients which, in turn, is likely to increase postoperative personal well-being and might save considerable medical and societal costs.

For a valid assessment of surgical fear two aspects need consideration: the selection of an adequate instrument and the timing of assessment. Previous studies have used a variety of instruments for the assessment of surgical fear: general instruments such as the State Trait Anxiety Inventory (STAI) ${ }^{7}$ and Hospital Anxiety and Depression Scale $(\mathrm{HADS})^{8}$ or disease specific instruments such as the Bypass Graft Fear Scale (BGFS)., 10 This publication will focus on another questionnaire, dedicated to assess surgical fear across all types of clinical and day surgery patients, the Surgical Fear Questionnaire (SFQ). ${ }^{11}$ The SFQ is formatted as an eight item index of surgical fear which can be divided into fear of short- and long-term consequences of surgery. Initial validation of the SFQ showed it to be a valid and reliable questionnaire, however, so far all studies administered the questionnaire at just one time point. As a result, information about the optimal time point for assessing surgical fear, the sensitivity of the SFQ to detect changes in fear over time, and factors that may influence the course of fear, is lacking. Therefore, in the present study we administered the SFQ at multiple time points before cataract surgery, i.e. one week before the scheduled procedure, the night before and at the morning of surgery. Boker et al. (2002) found a significant increase in surgical fear in patients undergoing various types of surgery after admittance to the surgical ward, compared to assessment during their visit to the pre-anesthesia clinic. ${ }^{12}$ However, a previous study in cataract patients did not find a difference in state anxiety one to two weeks before surgery compared to just before surgery. ${ }^{13}$ In the latter study, a generic state anxiety instrument was used, and the SFQ may prove more sensitive to detect subtle changes in surgery-related fear specifically. Moreover, since most cataract patients are scheduled for bilateral cataract surgery, this enables comparison between surgical fear of the first versus the second cataract surgery. Usually recovery is unproblematic and most patients feel relieved after surgery. ${ }^{13}$ We expected an increase in fear from one week before surgery to the assessment the night and the morning before surgery. In addition we expected that fear before the second surgery would be lower than before the first surgery.

Besides information on the validity and sensitivity of the SFQ with regard to effects of time course, we assessed convergent validity of the SFQ with other self-report instruments (numerical rating scale of fear, expected pain, and expected recovery) and with biological indices of stress (cortisol, alpha-amylase). Cortisol is used as a biomarker of activity of the hypothalamic-pituitary-adrenal (HPA) axis. ${ }^{14}$ Cortisol secretion shows a 
circadian rhythm with high morning levels and declining levels during the day; its secretion can be increased by physical or mental stress. We hypothesized that SFQ scores would predict cortisol elevation on the day of surgery compared to cortisol level on a control day. Cortisol can be measured in saliva where it serves as a good indicator of the serum unbound and active cortisol fraction. Salivary alpha-amylase (SAA) can be used as an indicator of activation of the autonomic nervous system. Previous studies showed good correlation between SAA levels and acute stress. ${ }^{15,16}$ We hypothesized that SFQ scores would predict a greater increase in pre- to postoperative sAA levels.

As a secondary aim, we assessed predictors of the level and time course of fear. Based on the findings of previous studies, we expected fear to be higher in women, in patients with high levels of preoperative pain, in patients with generally high psychological vulnerability, and in patients reporting low social support. ${ }^{11,13,17-19}$ Age, employment status, and educational level were included as additional factors that might be related to fear level and course, since these have been found predictive of surgical fear in some studies. ${ }^{11}$

In sum, we assessed sensitivity of the SFQ to detect differences in the preoperative time course of fear and sensitivity to detect changes in fear from first to second surgery. We hypothesized fear to increase closer to the time of surgery, and to be lower before the second surgery. Convergent validity was assessed by establishing the association of the SFQ with related self-report measures and with biological indices of stress. Finally we examined potential predictors of the level and time course of surgical fear.

\section{MATERIALS AND METHODS}

\section{Ethics statement}

Prior to the conduction of this study, approval was obtained from the Medical Ethics Committee of the Maastricht UMC+, Maastricht, the Netherlands. Signed informed consent was obtained from all participants and the study was conducted according to the declaration of Helsinki. This study was registered at the Dutch Trial Register under number NTR4491.

\section{Patient recruitment and sample size}

Patient recruitment took place at the University Eye Clinic at the Maastricht UMC+, Maastricht, the Netherlands. Inclusion criteria were age over 18 years, scheduled for elective cataract surgery in a day surgery setting, (loco-)regional anesthesia, a good command of the Dutch language, an ASA classification varying from I to III, and time to surgery of over 7 days. Exclusion criteria consisted of previous cataract surgery on the contralateral eye during the past year, illiteracy, cognitive impairment, surgery sched- 
uled under general anesthesia, ASA classification IV, Morbus Cushing, Addison's disease, hypothyroidism, hyperthyroidism, corticosteroid use (except inhaler), and participation in another trial.

If the true difference between mean SFQ scores assessed one week before surgery and assessed on the morning on the day of surgery is five, we would need to study 93 subjects in order to be able to reject the null hypothesis that this response difference is zero with a power set at 0.8 . Based on prior data ${ }^{11,20}$ we assumed a standard deviation of 17 for the difference between SFQ scores. The Type I error probability associated with this test of this null hypothesis was 0.05 . With an additional nine patients for correction of drop-out, 102 patients would need to be included.

\section{Measurements}

Surgical Fear Questionnaire (SFQ). The SFQ is an eight-item instrument for the assessment of self-reported surgical fear, suitable for general use among all types of adult surgery patients, covering a range of short-term (SFQ-s, item 1-4) and long-term (SFQ-I, item 5-8) surgery-related fears. All items are scored on an eleven point numeric rating scale (NRS) ranging from 0 (not at all afraid) to 10 (very afraid). This results in a score of 0-40 for each subscale and a total score of 0 to 80 . Items are: afraid of operation, anesthesia, postoperative pain, side effects, health deterioration, failed operation, incomplete recovery, and long duration of rehabilitation. See S1 File.

\section{Measures for assessing convergent validity}

Fear NRS. Pre- and post-operative fear was assessed with one question: "Circle the number that best reflects how afraid you are right now", that was scored on a 11-point scale (0-10). Labels were not at all afraid (0) and very afraid (10).

Expected pain. The level of pain patients expected to have after surgery was assessed with the question "Please indicate on a scale from 0 (no pain at all) to 10 (worst pain imaginable) how much pain you expect to feel four days after surgery". It was also scored on a 11-point scale and assessed at baseline, one week before surgery. ${ }^{21}$

Expected recovery. Expected recovery after surgery was also assessed at baseline, using the question "Please indicate on a scale from 0 to $100 \%$ to what extent you expect to be recovered from the operation, four weeks after the operation. 100\% recovery means that your health is back at the level it was before you developed your complaints and surgery was performed". This question was adapted from the Global Surgical Recovery scale (GSR). ${ }^{22}$ Another measure of expected recovery was the anticipated number of days until resumption of daily activities. Patients were asked at baseline to enter a number of days in answer to the question: "After how many days do you expect to be able to completely resume your daily activities?"

Cortisol and alpha-amylase. Saliva samples were obtained using Salivette swabs (Sarstedt AG \& Co., Nümbrecht, Germany). The synthetic swabs were specifically de- 
signed for cortisol determination in a small amount of saliva. Soft chewing on the swab during 60 seconds stimulates salivary production and ensures absorption of a sufficient amount of saliva. For the saliva samples collected at home, patients were instructed to collect the morning samples immediately after awaking, before brushing teeth or having breakfast, and for the other samples to refrain from smoking, eating and drinking for a minimum of 30 minutes, and in case of alcohol intake a minimum of two hours, prior to sample collection. Patients were asked to store the swabs in their home freezer before handing them in at the hospital visits. In the hospital saliva samples were frozen and stored in a freezer at $-20^{\circ} \mathrm{C}$ until shipment to and analysis at the laboratory of the Technical University of Dresden, Germany. After thawing, Salivettes were centrifuged at 3,000 rpm for 5 minutes, resulting in a clear supernatant of low viscosity. Salivary concentrations were measured using commercially available chemiluminescence immunoassay with high sensitivity (IBL International, Hamburg, Germany). The intra- and interassay coefficients of variation for cortisol were below $8 \%$.

The Salivettes additionally allowed for salivary alpha-amylase (SAA) assessment, performed in the same lab in Dresden. Concentration of alpha-amylase in saliva was measured by an enzyme kinetic method, as described by Rohleder et al. (2006). ${ }^{23}$

\section{Measures for examining predictors of surgical fear}

Sociodemographic data. A baseline questionnaire completed one week before surgery assessed age, sex, marital state (living alone versus living together/married), employment state (full-time or part-time work, temporary unable to carry out paid job, retired, student, unemployed, disabled, housekeeping, other), educational level (no, lower, intermediate, higher, university), smoking (yes/no/stopped), alcohol use (yes/no), and medication use. The following groups of medications were considered for the analyses of surgical fear and cortisol: total number of home medication, COX-2 inhibitors, antidepressants, anxiolytics, hypnotic sedatives, nonsteroidal anti-inflammatory drugs, inhaled steroids, antihypertensives, anticonvulsants. ${ }^{24}$

Pre-surgical pain. Pain present prior to surgery was assessed separately for pain related and unrelated to the cataract surgery. At first patients were asked whether they had pain last week or not. If so, a pain intensity score reflecting any pain (related or unrelated to the eye) at the moment of completing the questionnaire was assessed with a 0-10 numerical rating scale. ${ }^{21}$ The following baseline pain data were also assessed but not further analyzed: average and highest pain intensity during the last week, the onset of pain, the location of pain, and in addition to analgesic use, nonpharmaceutical pain treatment.

Social support. Social support was measured with the Medical Outcomes Study Social Support Survey (MOS-SSS) questionnaire. ${ }^{25}$ The MOS-SSS is a 19-item self-report questionnaire with four support subscales: emotional/informational, tangible, positive interaction, and affectionate support. In addition an overall support index can be calculated. One extra question assesses the number of close friends or relatives. Items are 
scored on a five-point scale. For the present study we used the total score, which is scaled from 0-100. Social support served as one of the control factors for stress, based on the assumption that patients with more social support might experience lower stress levels.

Stress. Stress was assessed at baseline using the Perceived Stress Scale (PSS). ${ }^{26}$ The PSS is a valid and reliable 10-item self-report measure of the degree to which life situations are appraised as stressful over the past month. Items are scored on a five-point scale. After rescaling of four positively worded items, a total score from 0-40 can be obtained by summation of the item scores. In addition, on the day of surgery patients were asked whether they had experienced a major event that caused stress in the week before surgery. Answer categories were no; yes, a little bit stress; yes, much stress; yes, very much stress.

\section{Other per-and postoperative measures}

Day of surgery. Body mass index (BMI), American Society of Anaesthesiologists (ASA) classification, co-morbidity, type of surgical intervention, type of local analgesia, duration of operation, perioperative complications, and unplanned hospital admission were assessed. Only data on ASA and hospital admission are reported.

Post-surgery questionnaire. At the final visit four weeks post-surgery patients handed in a final questionnaire completed one day earlier, assessing recovery as measured with the GSR. Patients responded to the question how much they felt recovered compared to their situation before surgery (0-100\%). Patients were also asked if they were able to perform their daily activities again since the last surgery, and if true, the number of days until that moment. Finally, postoperative pain intensity was assessed using the NRS.

Furthermore we retrospectively assessed whether patients would like to have had additional therapy to treat their surgical fear. The question was: "Thinking back to the period from one week before surgery until the day of surgery: would you have appreciated any treatment of surgical fear?" Possible answers were: "No, I was not (very) afraid and I don't appreciate treatment", No, I was afraid, however I don't need treatment", or "Yes, I was afraid and would have appreciated treatment". Patients who answered "yes" were able to choose one or more of the following treatment categories they would have preferred: more information provided by the ophthalmologist or the anesthesiologist, counseling by a psychologist, relaxation exercises, guided visualization, meditation, pharmaceutical treatment, or other.

To control for events that might affect the cortisol levels, the occurrence of major stressful events other than the operation during the four postoperative weeks was assessed. Participants were asked: "during the past four weeks (after surgery), did you go through anything that caused a lot of stress?" Response categories were "no"; "yes, but just a little bit of stress"; "yes, much stress"; "yes, very much stress". 


\section{Procedure}

Patients received a batch of date- and time-marked questionnaires and Salivette swabs the day they signed their informed consent form and the day of their first surgery. They were instructed to fill out the questionnaires and use the swabs at the indicated days and times, and for control, to register the exact dates and times of completion and saliva collection. The first SFQ was filled out on the evening one week before surgery (T1). At this time, patients also completed the baseline questionnaire. The second SFQ assessment took place on the evening before surgery (T2). On both of these days, patients were asked to collect three saliva samples, i.e., directly after awakening, in the afternoon between three and four o'clock, and in the evening between eight and nine. The third SFQ was filled out at home after awaking, on the morning surgery took place (T3). At this time, patients also scored fear on the NRS and took another saliva sample. In the hospital, two more NRS fear ratings were made, and at the same time saliva samples were taken: before surgery immediately after arrival at the ward of the University Eye Clinic, and after surgery when the patient had returned to the ward. Patients scheduled for cataract surgery on both eyes filled out the SFQ a fourth time, i.e., the evening before their second surgery (T4). Finally all patients returned to the hospital four weeks after (second) surgery for a control visit. The day before this control visit patients filled out the post-surgery questionnaire, containing questions on recovery, pain, and need for treatment of surgical fear (T5). Also, saliva samples were taken on that day, to serve as a baseline reference for cortisol. For an overview of the procedure see figure 1.

Cortisol was analyzed from the three saliva samples obtained on the two preoperative days, the morning sample and pre- and post-surgical samples on the day of the (first) surgery and from the three samples of the control day four weeks following surgery. Because sAA is thought to increase mainly in response to acute stressors, sAA was only analyzed in the immediate pre- and post-surgical samples on the day of surgery.

Data obtained by questionnaires were entered into a database in duplo by independent members of the study staff. Both databases were compared and all inconsistencies were corrected. 


\section{Time point 1}

1 week before first eye surgery, at home:

-SFQ: evening

-Baseline variables

-Expected recovery and postoperative pain

-Salivary cortisol: morning / afternoon / evening

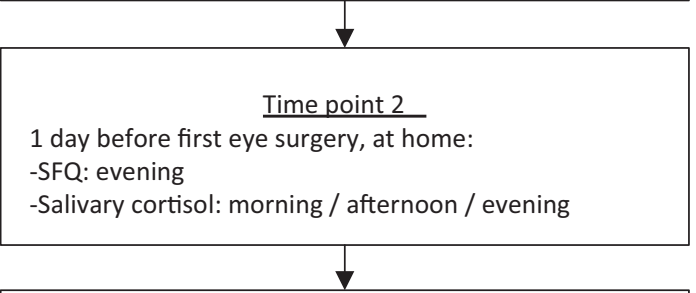

Time point 3

day of first surgery, at home:

-SFQ: morning

-NRS fear: morning

-Major stressful event during T1-T3

-Salivary cortisol: morning

day of first surgery, in hospital:

-NRS fear: pre- and postoperative

-Salivary cortisol: pre- and postoperative

-Salivary alpha amylase: pre- and postoperative

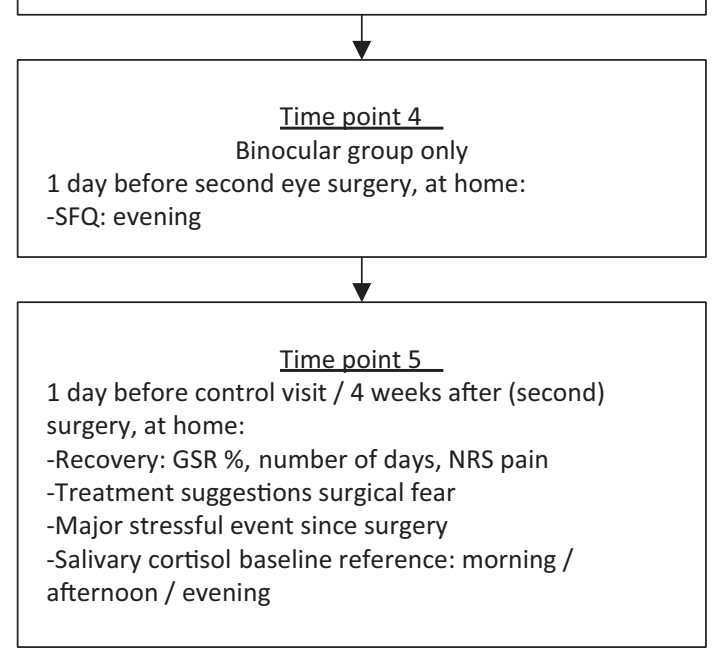

Figure 1. Schedule study measurements 


\section{Statistical analysis}

Descriptive analysis was performed on baseline and outcome data, using mean \pm SD, median $\left(25^{\text {th }}-75^{\text {th }}\right.$ percentile), number $(\%)$, or boxplots. To evaluate SFQ-S and SFQ-I scores over time bivariate analyses were performed using paired $t$-tests. We assessed whether there was an increase between T1 (one week before surgery) and T3 (the morning on the day of surgery), and for the binocular group we assessed whether there was a decrease between first surgery (T2, the evening before first surgery) and second surgery (T4, the evening before second surgery).

Convergent validity of the SFQ was assessed by calculating Pearson's correlation coefficients between SFQ-S and SFQ-I at T3 and the preoperative NRS fear scores, also completed at home at T3. Furthermore correlations were assessed between SFQ-S and SFQ-I at T1, and expected pain, expected recovery (GSR), and expected number of days until resumption of daily activities. To assess convergent validity of the SFQ with the biomarkers cortisol and SAA, multivariate analyses of natural-log transformed cortisol and SAA levels were performed. For cortisol, the differences between the morning samples of the day of surgery and the control day 4 weeks later (T3-T5) were regressed on SFQ-S and SFQ-I in separate analyses. For SAA, the differences between the immediate pre- and post-surgical levels were calculated and regressed on SFQ scores. Potential confounding factors such as sociodemographic variables, smoking habits, health status (defined by ASA-classification), medication use (total number of home medications, hypnotic sedatives, inhaled steroids), and the time at which the sample was taken were added to the model. ${ }^{27,28}$ Results of the regression analyses are presented as beta (SD).

To examine predictors of the overall level and the time course of surgical fear before the first surgery, multivariate analysis was performed using linear mixed modeling with a random person intercept and time variables defining $\mathrm{T} 2$ and $\mathrm{T} 3$, while $\mathrm{T} 1$ served as a reference. Age, sex, employment status (recoded to paid job yes/no), educational level (recoded to no/lower, intermediate, or higher/university education), pain at baseline (NRS $\geq 4$ versus $<4$ ), social support (total score), perceived stress during the month prior to T1 as measured with the PSS, and stress during the week between T1-T3 (no/little stress versus (very) much stress) were included as predictor variables. We also included the use of anxiolytics and antidepressants as reported on the medical file as additional indicators of psychological vulnerability. Interaction effects between time and each of the selected variables were assessed per variable, for SFQ-S and SFQ-I separately. In the case of a significant interaction effect, the interaction terms were added to the multivariate model (criterion $p<0.10$ ). The time variables, age, and sex were entered using a forced entry method, for all other variables a backward deletion procedure was used (criterion $p<0.10$ ). A final selection criterion of $p<0.05$ was used. A significant interaction with time reflects an effect of the respective variable on time course, while a significant main effect implies an overall effect of the respective variable on surgical fear, irrespec- 
tive of time. Results are presented as estimate (SD), which reflect the change in outcome for each unit of change in the predictor.

A post-hoc analysis was performed to assess whether the SFQ-S and SFQ-I were related to patients' need for preoperative treatment of fear. Using independent $t$-tests, the SFQ-S and SFQ-I scores of patients indicating a need for additional psychological treatment for surgical fear were compared to the scores of patients not reporting this need. For all analyses a final $p$-value $<0.05$ was considered statistically significant. Analyses were performed using IBM SPSS Statistics for Windows, version 23.0. Armonk, NY, USA.

\section{RESULTS}

Between March 2014 and January 2015109 cataract surgery patients were included, of whom 98 (89.9\%) were suitable for analyses. Detailed information on patient flow is presented in figure 2. Mean (SD) age of the participants was 68.8 (8.9) years, 42 (42.9\%) were female, and 49 (50.5\%) were scheduled for cataract surgery on both eyes. Patients who underwent cataract surgery on the contralateral eye during the last year were excluded, however there were 17 (17.7\%) patients who reported other eye surgery during the last year. Detailed information on the baseline characteristics is presented in table 1.

Postoperative recovery was generally rated as good. Four weeks after cataract surgery perceived recovery on the GSR was $89.9 \%$ (SD 17.7), average pain in the eye during the last week was 0 (median, $25^{\text {th }}-75^{\text {th }}$ percentile $0-1$ ), 83 patients $(89.2 \%$ ) were able to perform their daily activities again (missing data 5), and the number of days before they were able to perform daily activities again since the last surgery was 6.9 (7.1). There were no unplanned hospital admissions. 


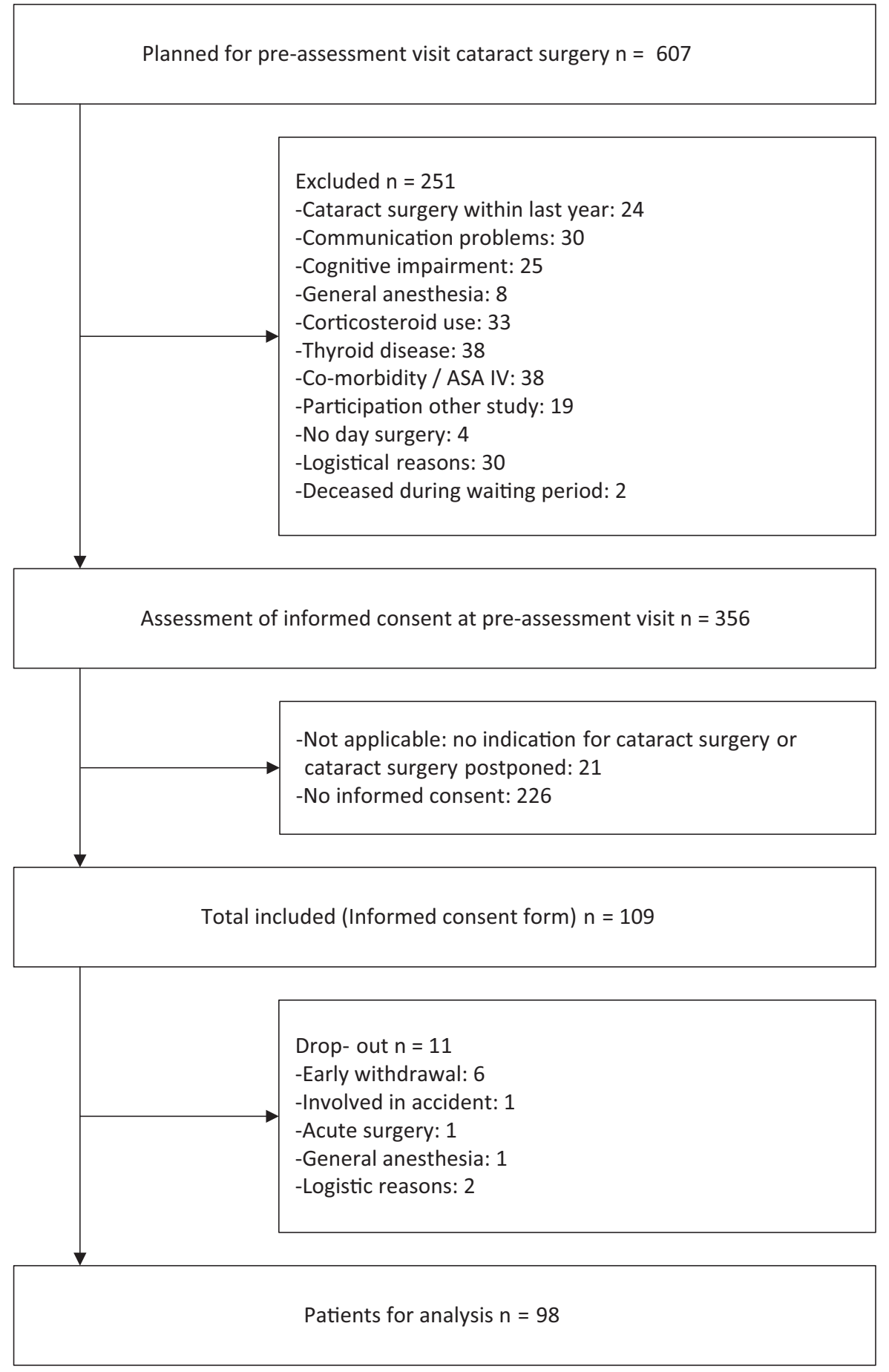

Figure 2. Patient flow 
Table 1. Baseline characteristics

\begin{tabular}{|c|c|}
\hline Variable & $\mathrm{N}=98$ \\
\hline Age & $68.8(8.9)$ \\
\hline \multicolumn{2}{|l|}{ Sex } \\
\hline male & $56(57 \%)$ \\
\hline female & 42 (43\%) \\
\hline \multicolumn{2}{|l|}{ Education } \\
\hline no / lower & $17(18 \%)$ \\
\hline intermediate & $36(38 \%)$ \\
\hline higher & $42(44 \%)$ \\
\hline missing & 3 \\
\hline \multicolumn{2}{|l|}{ Employment } \\
\hline paid job & $20(21 \%)$ \\
\hline no paid job & $76(79 \%)$ \\
\hline missing & 2 \\
\hline \multicolumn{2}{|l|}{ Marital status } \\
\hline living alone & $27(28 \%)$ \\
\hline living together & $69(72 \%)$ \\
\hline missing & 2 \\
\hline Expected pain (0-10) & $2.0(0-3)$ \\
\hline Expected GSR (0-100\%) & $91.9(14.4)$ \\
\hline Expected number of days & $14.0(14.7)$ \\
\hline MOS-SSS total (0-100) & $78.2(23.5)$ \\
\hline PSS (0-40) & $10.9(6.7)$ \\
\hline \multicolumn{2}{|l|}{ Non-surgical stress } \\
\hline no & $52(56 \%)$ \\
\hline a bit & 27 (29\%) \\
\hline a lot & $12(13 \%)$ \\
\hline extreme & $2(2 \%)$ \\
\hline missing & 5 \\
\hline \multicolumn{2}{|l|}{ ASA } \\
\hline 1 & $20(21 \%)$ \\
\hline$\|$ & $65(66 \%)$ \\
\hline III & $13(14 \%)$ \\
\hline \multicolumn{2}{|l|}{ Smoking } \\
\hline no & $31(32 \%)$ \\
\hline stopped & $59(62 \%)$ \\
\hline yes & $6(6 \%)$ \\
\hline missing & 2 \\
\hline \multicolumn{2}{|l|}{ Alcohol } \\
\hline yes & $76(79 \%)$ \\
\hline no & $20(21 \%)$ \\
\hline missing & 2 \\
\hline \multicolumn{2}{|l|}{ Medication } \\
\hline anxiolytics & $5(5 \%)$ \\
\hline antidepressants & $5(5 \%)$ \\
\hline hypnotic sedatives & $6(6 \%)$ \\
\hline inhaled corticosteroids & $7(7 \%)$ \\
\hline antihypertensives & $44(45 \%)$ \\
\hline Previous eye surgery last year & $17(18 \%)[2]$ \\
\hline
\end{tabular}




\begin{tabular}{ll}
\hline Variable & $\mathrm{N}=98$ \\
\hline Planned cataract surgery & $48(49 \%)$ \\
monocular & $49(51 \%)$ \\
binocular & 1 \\
missing & \\
Preoperative pain & $2(2 \%)[1]$ \\
related to eye & $36(37 \%)[1]$ \\
\hline
\end{tabular}

Mean (SD), median $\left(25^{\text {th }}-75^{\text {th }}\right.$ percentile), number (\%), [missing].

Expected pain: expected pain four days after surgery (numeric rating scale, NRS 0-10); Expected GSR: expected global surgical recovery at four weeks after last surgery (T5); Expected number of days: expected number of days until full recovery after last surgery. MOS-SSS number: medical outcome study social support scale, number of close friends/relatives; MOS-SSS total: total score (0-100). PSS: perceived stress scale; Nonsurgical stress: stress unrelated to surgery with major impact during the week before surgery (the week of T1T3). ASA: American Society of Anesthesiologists. Preoperative pain: pain last week, yes/no.

\section{Time course of surgical fear}

Our data revealed low surgical fear scores at all time points for most patients. A minority of patients did however report considerable levels of fear, with a maximum of 36 for both SFQ-S and SFQ-I at T3. Mean (SD) SFQ-s scores were 8.2 (8.4) at T1 and 8.6 (8.7) at T3. The SFQ-I scores were $6.9(8.1)$ at T1 and 8.1 (8.5) at T3. A paired $t$-test revealed that the increase between T1 and T3 was significant for SFQ-I $(p=0.034)$ but not for SFQs. At T2, the evening before the first surgery, the SFQ-s score of the total sample was 8.7 (8.5), SFQ-I was 7.7 (7.7). For the binocular group SFQ-s at T2 was 9.0 (SD 8.6) and decreased to $5.3(5.8, p=0.002)$ at $T 4$, the evening before the second surgery. SFQ-I decreased from $8.0(7.7)$ to $5.6(6.2, p=0.010)$. Details on SFQ results are presented in the boxplots of figure 3 .

\section{Convergent validity of the SFQ with NRS fear scores and preoperative expectations}

On the day of surgery the NRS fear score at home was 2.6 (2.6) and pre- and postoperative in-hospital scores were 2.7 (2.4) and 0.8 (1.4) respectively. Convergent validity of the SFQ was assessed using the preoperative NRS fear score assessed at home, and outcome expectancy scores. The Pearson correlations between SFQ-s and SFQ-I scores at T3 and preoperative NRS fear were high, 0.898 and 0.828 , and significant at alpha $=0.01$ level. The correlations of SFQ scores of T1 with expected pain were somewhat lower, 0.543 for SFQ-S and 0.493 for SFQ-I, but also significant at alpha $=0.01$ level. Finally, negative correlations with expected recovery were found, -0.267 significant at alpha $=0.05$ level for SFQ-S and -0.439 for SFQ-I, significant at alpha $=0.01$ level. The expected number of days until reuptake of activities did not correlate with any of the SFQ scores; no data shown. 


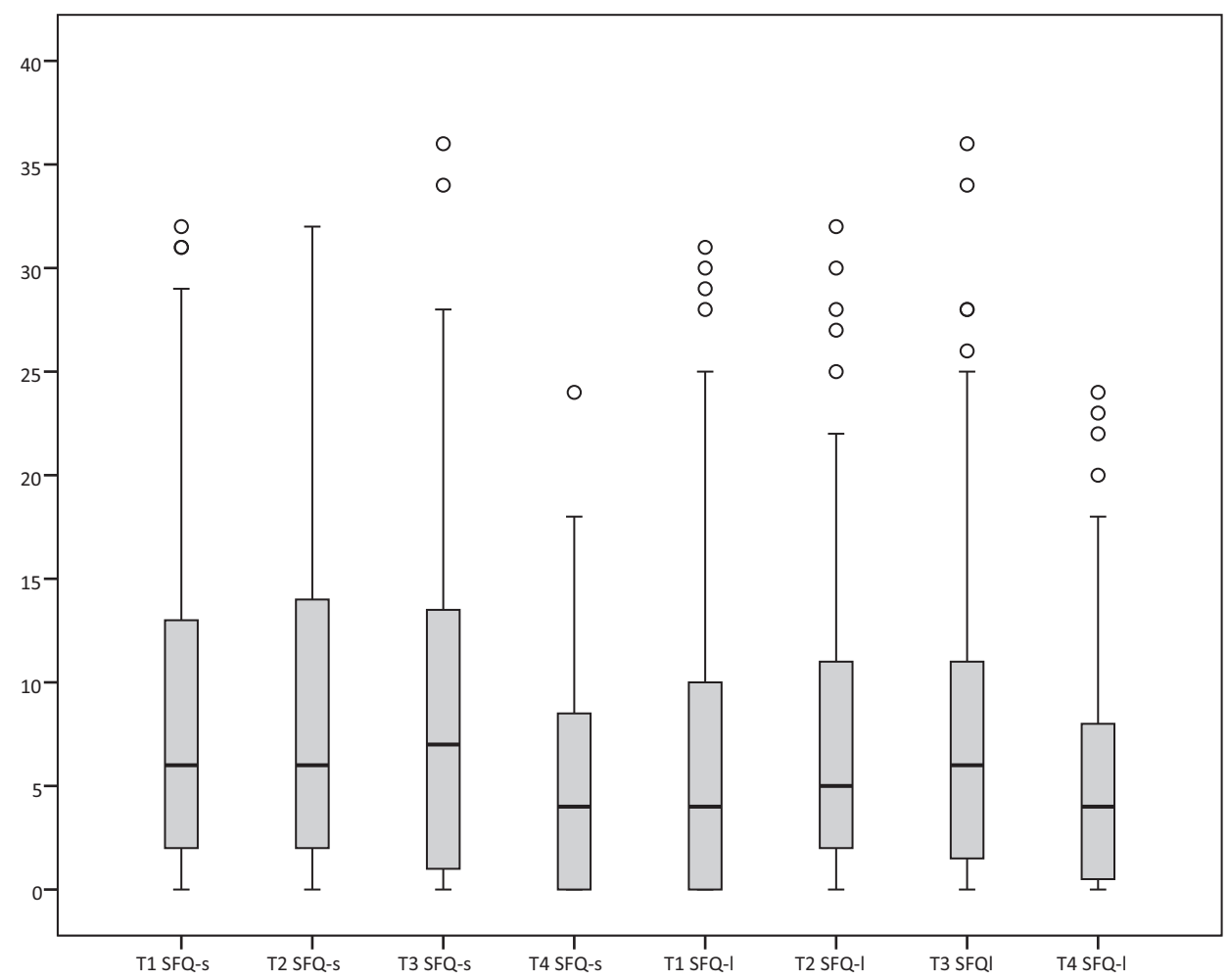

Figure 3. Surgical fear short- and long-term subscales, at time point 1-4 T1-3: all patients, T4: binocular patients only.

T1: one week before surgery, T2: the evening before surgery, T3: the morning of the day of surgery, morning, at home, T4: binocular patients, the evening before their second surgery. ${ }^{\circ}$ indicates outlier.

\section{Convergent validity of the SFQ with salivary cortisol and alpha-amylase}

The cortisol levels sampled at T1-3 and T5 showed normal circadian patterns with levels decreasing by approximately $2 \mathrm{nmol} / \mathrm{L}$ per hour from morning through evening measures. Mean collection times complied with the given patient instructions, see S1 figure and S1 table. On average, the samples were collected one hour (SD 1.3) earlier on the day of surgery compared to the day before the control visit. Linear regression analyses were performed using the calculated difference between the natural-log transformed cortisol sample collected at home on the day of surgery and the morning cortisol sample of the day before the control visit as dependent variable, and SFQ scores as independent variables. Furthermore we corrected for potential confounders by including age, smoking, medication use (hypnotic sedatives, inhaled corticosteroids), ASAclassification, and both cortisol sampling times in the analyses. Analyses were performed separately for SFQ-S and SFQ-I. Change in cortisol level was predicted by time of day of the saliva collected at the day of surgery (T3 morning sample). Neither SFQ-s or 


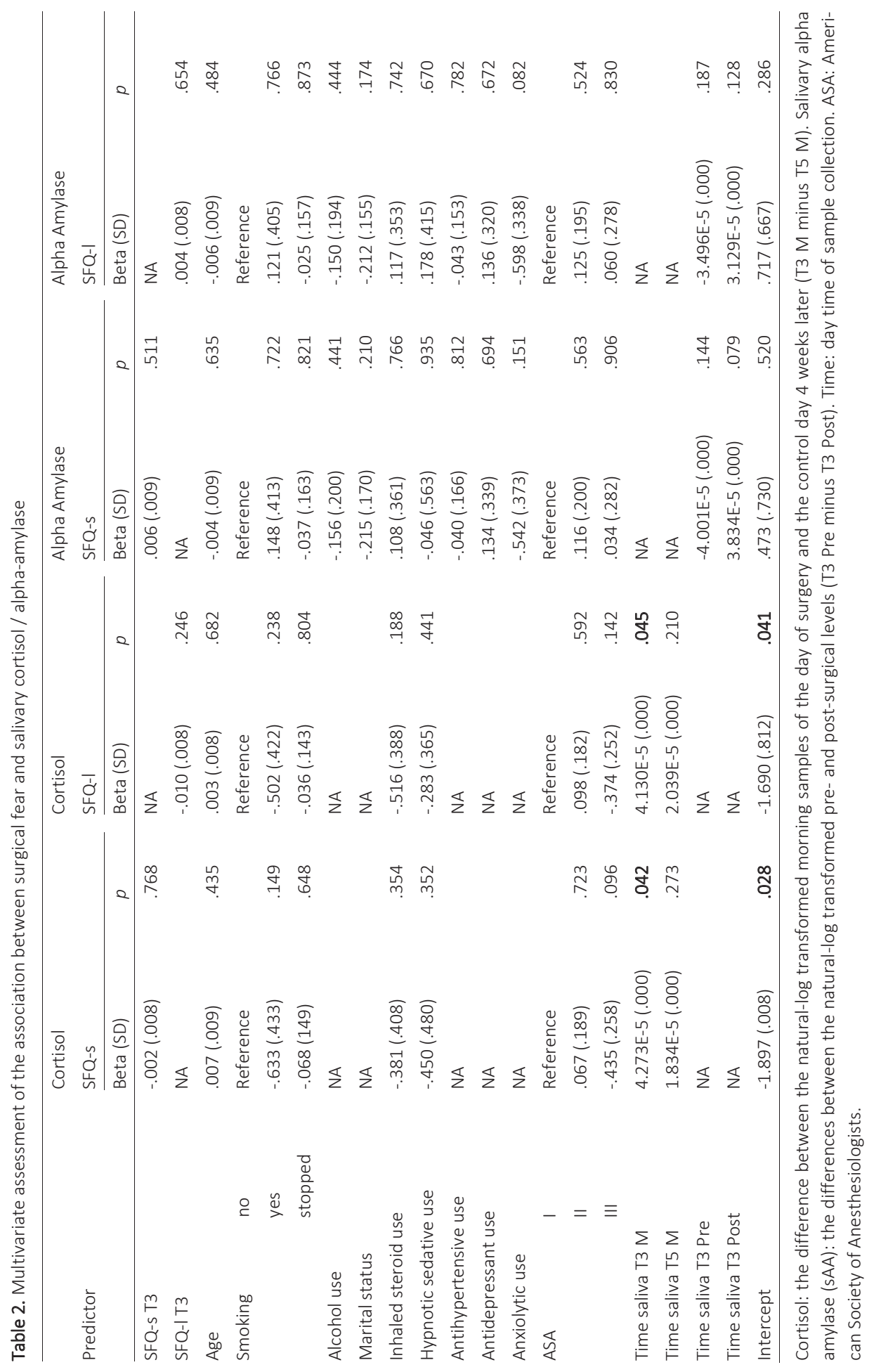


SFQ-I, nor any of the mentioned confounders contributed significantly to the model, see table 2. Additional correction for the potential confounders stress and social support did not change these findings. Also the association between the SFQ and the cortisol samples collected at other time points was explored, however no association was found.

The preoperative SAA level at T3 was 196.5 (158.3) U/ml mean (SD) and increased to 258.7 (197.1) postoperatively. A paired $t$-test on the natural-log transformed data revealed that this increase was significant, $t-4.628 d f 85 p<0.001$. Linear regression analysis was performed with the calculated difference between the natural-log transformed pre- and postoperative sAA sample as dependent variable. Also for SAA, assessed at the day of surgery, separate analyses were performed for SFQ-S and SFQ-I. We controlled for age, marital status, smoking, alcohol use, medication use (antihypertensives, inhaled corticosteroids, hypnotic sedatives, antidepressants, anxiolytics), health status (ASAclassification), and both sAA sampling times. We found no association between either surgical fear or any of the other co-variables with perioperative change in SAA level, see table 2 .

\section{Prediction of the level and course of fear}

The following variables showed an interaction with time in their association with at least one of the subscales of the SFQ in bivariate analyses, and were therefore added to the multivariate model: educational level, perceived stress during the month before T1, stress during the week between T1-T3, use of antidepressants, and use of anxiolytics. The final results after a backward deletion procedure, for SFQ-S and SFQ-I are presented in table 3. For SFQ-s significant main effects were found for baseline pain [6.0 (2.2), $p=0.007]$ and perceived stress during the month before surgery [0.5 (0.1), $p<0.001]$. Patients reporting higher pre-surgical pain and stress levels reported overall higher levels of surgical fear. Furthermore the effect of time (T3 versus T1) on surgical fear was different for patients with no/lower education compared to the reference group, patients with higher/university education [estimate -2.9 (1.3), $p=0.025$ ]. For patients using antidepressants the effect of time (T2 versus T1) on SFQ-s was larger compared to patients not using antidepressants [6.8 (2.1), $p=0.001]$.

Significant main effects on SFQ-I were found for baseline pain and perceived stress during the month preceding T1. Similar to the findings with SFQ-S, baseline pain [8.6 (2.1), $p<0.001]$ and perceived stress during the month before surgery [0.5 (0.1), $p<0.001]$ were associated with higher SFQ-I scores. Also similar to SFQ-S, we found different effects of time in relation to educational level and use of antidepressants. The effect of time (T3 versus T1) for patients with no/lower educational level was opposite to that of the higher/university level group [estimate -3.3 (1.3), $p=0.013$ ]. The effect of time (T2 versus T1) for patients with intermediate educational level was opposite to that of the higher educational level group [estimate $-2.2(1.0), p=0.037$ ]. For patients using antidepressants the effect of time (T2 versus T1) on SFQ-I was larger compared to 
patients not using antidepressants [8.0 (2.3), $p=0.001]$. Finally, for patients reporting much/very much stress resulting from a major event in the week before surgery, the effect of time (T3 versus T1) on SFQ-I was larger compared to patients with no or little stress [3.6 (1.3), $p=0.008]$.

Table 3. Predictors of surgical fear

\begin{tabular}{|c|c|c|c|c|}
\hline & SFQ-S & & SFQ-I & \\
\hline Predictor & Estimate (SD) & $p$ & Estimate (SD) & $P$ \\
\hline \multicolumn{5}{|l|}{ Time } \\
\hline T1 & Reference & & Reference & \\
\hline $\mathrm{T} 2$ & $0.18(.69)$ & .795 & $1.51(.72)$ & .036 \\
\hline T3 & $.86(.69)$ & .214 & $1.66(.71)$ & .021 \\
\hline Age & $.03(.09)$ & .758 & $-.11(.08)$ & .180 \\
\hline Sex & $-1.58(1.50)$ & .297 & $-1.59(1.53)$ & .300 \\
\hline \multicolumn{5}{|l|}{ Education } \\
\hline no/lower & $-.97(2.10)$ & .645 & $.15(2.17)$ & .946 \\
\hline intermediate & $-2.03(1.65)$ & .221 & $-.12(1.69)$ & .942 \\
\hline higher/university & Reference & & Reference & \\
\hline Baseline pain & $6.01(2.17)$ & .007 & $8.64(2.11)$ & $<.001$ \\
\hline MOS-SSS (0-100) & $-.06(.03)$ & .083 & NA & \\
\hline PSS baseline stress $(0-40)$ & $.48(.12)$ & $<.001$ & $.47(.12)$ & $<.001$ \\
\hline Antidepressants use & $3.64(3.48)$ & .299 & $1.24(3.61)$ & .733 \\
\hline Presurgical stress T1-T3 & NA & & $-4.34(2.25)$ & .056 \\
\hline \multicolumn{5}{|l|}{ Interaction with Time } \\
\hline T2 * no/lower education & $-1.15(1.30)$ & .379 & $-1.57(1.35)$ & .244 \\
\hline T3 * no/lower education & $-2.89(1.28)$ & .025 & $-3.27(1.29)$ & .013 \\
\hline T2 * intermediate education & $0.81(1.00)$ & .421 & $-2.15(1.02)$ & .037 \\
\hline T3 * intermediate education & $-.06(1.01)$ & .952 & $-1.66(1.02)$ & .104 \\
\hline T2 $*$ antidepressant use & $6.83(2.05)$ & .001 & $8.04(2.29)$ & .001 \\
\hline T3 $*$ antidepressant use & $1.42(2.05)$ & .488 & $1.54(2.28)$ & .499 \\
\hline T2 * presurgical stress T1-T3 & NA & & $.34(1.37)$ & .805 \\
\hline T3 * presurgical stress T1-T3 & NA & & $3.55(1.33)$ & .008 \\
\hline Intercept & $6.44(6.84)$ & .349 & $9.86(5.73)$ & .089 \\
\hline
\end{tabular}

$\mathrm{T} 1$ = one week before surgery, $\mathrm{T} 2$ = the day before surgery, $\mathrm{T} 3$ = the day of surgery.

Baseline pain = any pain at baseline (NRS $\geq 4)$. MOS-SSS = Medical Outcomes Study Social Support Survey. PSS baseline stress $=$ Perceived Stress Scale. Presurgical stress T1-T3 $=$ much/very much stress resulting from a major event in the week before surgery.

\section{Postoperative evaluation}

At T5, we assessed whether any form of preoperative treatment of surgical fear would be appreciated. Most of the patients $(72,77.4 \%)$ answered that they had not been afraid of the procedure and did not need any psychological treatment. Thirteen (14\%) 
patients indicated that they had been afraid, but did not need any psychological treatment. Finally, eight (8.6\%) patients indicated that they had been afraid and would have appreciated psychological treatment before surgery (five patients had missing data). However, 13 patients in total selected one or more of the proposed preoperative treatment options: more information from the ophthalmologist (8) or anesthesiologist (8), counseling by a psychologist (2), relaxation exercises (2), guided visualization (3), meditation (2), medicinal treatment (3), other (3). Interestingly, a post-hoc analysis revealed that all SFQ-S and SFQ-I scores at T1-3 of the 13 patients asking for preoperative treatment were significantly higher (mean SFQ-S and SFQ-I scores between 12.1 and 14.5) compared with the scores of the patients not asking for support (mean scores between 6.3 and 7.8 , all $p<0.05)$.

\section{DISCUSSION}

The results of this study provide further support for the validity of the SFQ. This is reflected by the high correlations with the fear NRS scores and the moderate but significant correlations with outcome expectancies. As hypothesized, the SFQ was capable of detecting changes in fear over a period from one week before surgery to the day of surgery (SFQ-I only), and in the binocular group also between fear of the first versus the second surgery. However, we found no association with the biological parameters cortisol or alpha-amylase.

Surgical fear levels assessed during the week before elective cataract surgery were low and fear of the short-term aspects of surgery remained stable. However, adequate sensitivity of the SFQ was shown by the detection of small but significant changes in fear over time for SFQ-I, before second eye surgery, and also by the finding that patients indicating any preference for treatment of surgical fear showed significantly higher fear scores. Because changes over time were small, these results suggest that surgical fear scores assessed at different time points in the week before surgery were comparable.

The generally low levels of surgical fear may be due to the refined preoperative procedure of patient assessment and patient information. ${ }^{29}$ Also the knowledge that cataract surgery currently is one of the ultimate routine surgical procedures, performed in a modern well-equipped day-surgery setting, might lower preoperative fear levels. Although one previous study on cataract patients reported preoperative STAI anxiety levels above normal compared to norm scores, ${ }^{30}$ our findings are in line with several other studies on cataract patients ${ }^{31,32}$ or fast-track trauma and plastic surgery ${ }^{33}$ that also reported low anxiety scores before surgery. The observed low fear scores might also have contributed to the fact that we did not find any association between SFQ and cortisol or alpha-amylase levels. It thus remains the question whether in a population with higher SFQ-scores an association would emerge. 
Our data reveal further evidence for the validity of the subscales of the SFQ. Interestingly, for expected recovery, the strongest correlation occurred with the SFQ-I subscale, which includes questions on fear of incomplete and long duration of recovery. The highest correlation with expected pain was found with the SFQ-S, which includes a question on fear of pain, however the difference with SFQ-I was relatively small.

As a secondary aim we examined predictors of the level and preoperative course of surgical fear. In line with previous findings, ${ }^{11}$ in this population preoperative pain was an overall factor strongly associated with increased SFQ-S and SFQ-I, together with perceived stress in the month before T1. Educational level affected the course of SFQ-s and SFQ-I over time, but the effect sizes were smaller compared to the effect of baseline pain and antidepressant use. Only one other study assessed educational level in relation to the course of surgical fear. This study reported that education had no effect on the course of fear. ${ }^{9}$ Previously reported main effects of educational level in relation to fear are conflicting. In one of the samples in our previous report, lower education was associated with lower fear whereas in the other samples lower educated patients had higher fear scores, albeit not significant across all samples. ${ }^{11}$ Lower education was also associated with higher fear in another study, ${ }^{34}$ whereas Ebirim found no differences in surgical fear prevalence between different educational levels. ${ }^{35}$ In our study the impact of antidepressant use was substantial. In patients using antidepressants larger effects of time on both SFQ-S and SFQ-I were found. This corresponds with previously described associations between anxiety and depression by other authors. ${ }^{36,37}$ An interrelationship between depression and preoperative anxiety assessed two weeks before surgery was found in a study in cardiac surgery patients. ${ }^{38}$ However, in contrast to our findings, depression two weeks before surgery was not associated with anxiety assessed one day before surgery.

\section{Strengths and limitations}

The strength of the current study is that further insight in the validity of the SFQ was obtained. The current study further confirms the congruent validity of the SFQ and additionally it reveals that the SFQ is sensitive to detect even small differences in fear, based on time course or other causes. The main limitation of this study is that, as a result of the low fear level in the study population, the ability to assess potential associations between the SFQ and biomarkers cortisol and SAA was restricted.

\section{Conclusions}

Considering the associations with other self-report measures of fear, expected pain and recovery, our data support the validity of the SFQ. Moreover, the SFQ is sensitive to detect small changes in the preoperative course of fear in the week before cataract surgery. However, we found no association between the SFQ and biomarkers cortisol or 
alpha-amylase. Common factors influencing the course in time of both SFQ-S and SFQ-I are educational level and antidepressant use. Irrespective of time span to surgery, pain and stress at baseline predict increased surgical fear. Except for certain subgroups as mentioned above, the observed variations in fear scores were clinically insignificant, suggesting that in general, surgical fear scores assessed at different time points in the week before surgery are well comparable.

\section{ACKNOWLEDGEMENTS}

We thank Heike van Schaik for the effective patient recruitment and data entry during her scientific traineeship at our department and Annick Ronden for her support with data entry and data cleaning. 


\section{REFERENCES}

1. Celestin J, Edwards RR, Jamison RN Pretreatment psychosocial variables as predictors of outcomes following lumbar surgery and spinal cord stimulation: a systematic review and literature synthesis. Pain Med. 2009; 10(4): 639-653.

2. Ip HY, Abrishami A, Peng PW, et al. Predictors of postoperative pain and analgesic consumption: a qualitative systematic review. Anesthesiology. 2009; 111(3): 657-677.

3. McKenzie LH, Simpson J, Stewart M A systematic review of pre-operative predictors of post-operative depression and anxiety in individuals who have undergone coronary artery bypass graft surgery. Psychol Health Med. 2010; 15(1): 74-93.

4. Rosenberger PH, Jokl P, Ickovics J Psychosocial factors and surgical outcomes: an evidence-based literature review. J Am Acad Orthop Surg. 2006; 14(7): 397-405.

5. Theunissen M, Peters ML, Bruce J, et al. Preoperative anxiety and catastrophizing: a systematic review and meta-analysis of the association with chronic postsurgical pain. Clin J Pain. 2012; 28(9): 819-841.

6. Wilson CJ, Mitchelson AJ, Tzeng TH, et al. Caring for the surgically anxious patient: a review of the interventions and a guide to optimizing surgical outcomes. Am J Surg. 2015.

7. Spielberger CD Assessment of state and trait anxiety: conceptual and methodological issues. South Psychol. 1985; 2: 6-16.

8. Zigmond AS, Snaith RP The hospital anxiety and depression scale. Acta Psychiatr Scand. 1983; 67(6): 361370.

9. Koivula M, Tarkka MT, Tarkka M, et al. Fear and anxiety in patients at different time-points in the coronary artery bypass process. Int J Nurs Stud. 2002; 39(8): 811-822.

10. Koivula M, Tarkka MT, Tarkka M, et al. Fear and in-hospital social support for coronary artery bypass grafting patients on the day before surgery. Int J Nurs Stud. 2002; 39(4): 415-427.

11. Theunissen M, Peters ML, Schouten EG, et al. Validation of the Surgical Fear Questionnaire in Adult Patients Waiting for Elective Surgery. PLoS One. 2014; 9(6): e100225.

12. Boker A, Brownell L, Donen N The Amsterdam preoperative anxiety and information scale provides a simple and reliable measure of preoperative anxiety. Can J Anaesth. 2002; 49(8): 792-798.

13. Nijkamp MD, Kenens CA, Dijker AJ, et al. Determinants of surgery related anxiety in cataract patients. Br J Ophthalmol. 2004; 88(10): 1310-1314.

14. Hellhammer DH, Wust S, Kudielka BM Salivary cortisol as a biomarker in stress research. Psychoneuroendocrinology. 2009; 34(2): 163-171.

15. Nater UM, Rohleder N Salivary alpha-amylase as a non-invasive biomarker for the sympathetic nervous system: current state of research. Psychoneuroendocrinology. 2009; 34(4): 486-496.

16. Schumacher S, Kirschbaum C, Fydrich T, et al. Is salivary alpha-amylase an indicator of autonomic nervous system dysregulations in mental disorders?--a review of preliminary findings and the interactions with cortisol. Psychoneuroendocrinology. 2013; 38(6): 729-743.

17. Koivula M, Paunonen-IImonen M, Tarkka MT, et al. Social support and its relation to fear and anxiety in patients awaiting coronary artery bypass grafting. J Clin Nurs. 2002; 11(5): 622-633.

18. Mols F, Denollet J Type D personality among noncardiovascular patient populations: a systematic review. Gen Hosp Psychiatry. 2010; 32(1): 66-72.

19. Pedersen SS, van Domburg RT, Theuns DA, et al. Type D personality is associated with increased anxiety and depressive symptoms in patients with an implantable cardioverter defibrillator and their partners. Psychosom Med. 2004; 66(5): 714-719.

20. Hoofwijk DM, Fiddelers AA, Peters ML, et al. Prevalence and Predictive Factors of Chronic Postsurgical Pain and Poor Global Recovery 1 Year After Outpatient Surgery. Clin J Pain. 2015; 31(12): 1017-1025.

21. Ferreira-Valente MA, Pais-Ribeiro JL, Jensen MP Validity of four pain intensity rating scales. Pain. 2011; 152(10): 2399-2404.

22. Kleinbeck SV Self-reported at-home postoperative recovery. Res Nurs Health. 2000; 23(6): 461-472. 
23. Rohleder N, Wolf JM, Maldonado EF, et al. The psychosocial stress-induced increase in salivary alphaamylase is independent of saliva flow rate. Psychophysiology. 2006; 43(6): 645-652.

24. Nicolson NA, Davis MC, Kruszewski D, et al. Childhood maltreatment and diurnal cortisol patterns in women with chronic pain. Psychosom Med. 2010; 72(5): 471-480.

25. Sherbourne CD, Stewart AL The MOS social support survey. Soc Sci Med. 1991; 32(6): 705-714.

26. Cohen S, Kamarck T, Mermelstein R A global measure of perceived stress. J Health Soc Behav. 1983; 24(4): 385-396.

27. Kudielka BM, Gierens A, Hellhammer DH, et al. Salivary cortisol in ambulatory assessment--some dos, some don'ts, and some open questions. Psychosom Med. 2012; 74(4): 418-431.

28. Rohleder N, Nater UM Determinants of salivary alpha-amylase in humans and methodological considerations. Psychoneuroendocrinology. 2009; 34(4): 469-485.

29. Nijkamp MD, Ruiter RA, Roeling $M$, et al. Factors related to fear in patients undergoing cataract surgery: a qualitative study focusing on factors associated with fear and reassurance among patients who need to undergo cataract surgery. Patient Educ Couns. 2002; 47(3): 265-272.

30. Gombos K, Jakubovits E, Kolos A, et al. Cataract surgery anaesthesia: is topical anaesthesia really better than retrobulbar? Acta Ophthalmol Scand. 2007; 85(3): 309-316.

31. Kim MS, Cho KS, Woo H, et al. Effects of hand massage on anxiety in cataract surgery using local anesthesia. J Cataract Refract Surg. 2001; 27(6): 884-890.

32. Moon JS, Cho KS The effects of handholding on anxiety in cataract surgery patients under local anaesthesia. J Adv Nurs. 2001; 35(3): 407-415.

33. Wetsch WA, Pircher I, Lederer W, et al. Preoperative stress and anxiety in day-care patients and inpatients undergoing fast-track surgery. Br J Anaesth. 2009; 103(2): 199-205.

34. Kindler $\mathrm{CH}$, Harms $\mathrm{C}$, Amsler $\mathrm{F}$, et al. The visual analog scale allows effective measurement of preoperative anxiety and detection of patients' anesthetic concerns. Anesth Analg. 2000; 90(3): 706-712.

35. Ebirim L, Tobin M Factors Responsible For Pre-Operative Anxiety In Elective Surgical Patients At A University Teaching Hospital: A Pilot Study The Internet Journal of Anesthesiology. 2010; 29(2): 6.

36. Clark LA, Watson D Tripartite Model of Anxiety and Depression - Psychometric Evidence and Taxonomic Implications. J Abnorm Psychol. 1991; 100(3): 316-336.

37. den Hollander-Gijsman ME, Wardenaar KJ, de Beurs E, et al. Distinguishing symptom dimensions of depression and anxiety: An integrative approach. J Affect Disorders. 2012; 136(3): 693-701.

38. Duits AA, Duivenvoorden HJ, Boeke S, et al. A structural modeling analysis of anxiety and depression in patients undergoing coronary artery bypass graft surgery: a model generating approach. J Psychosom Res. 1999; 46(2): 187-200. 


\section{SUPPORTING INFORMATION}

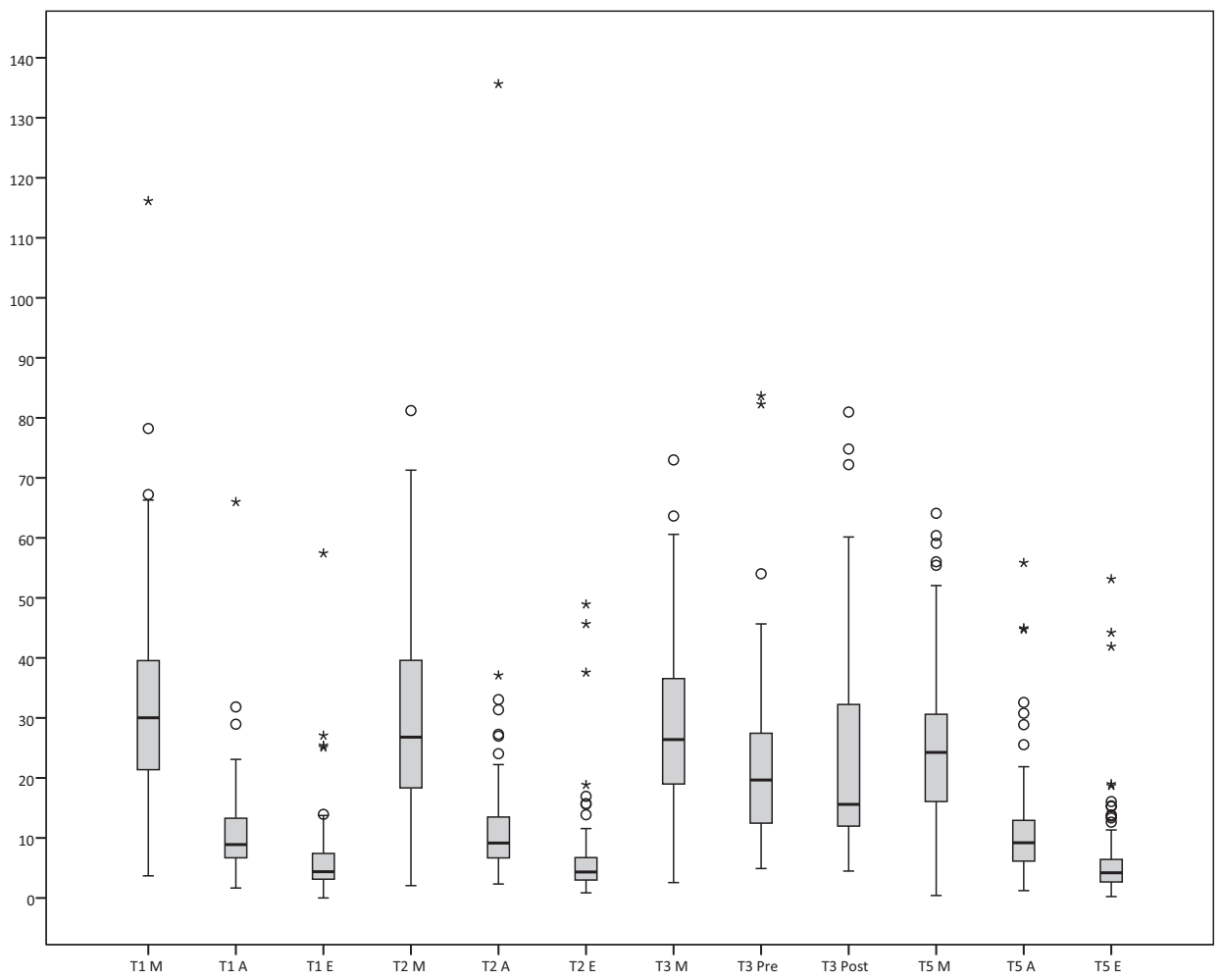

S1 Figure. Boxplot salivary cortisol

Salivary cortisol, $\mathrm{nmol} / \mathrm{L} .{ }^{\circ}$ and * indicate outlier and extreme outlier.

$\mathrm{T} 1$ = one week before surgery, $\mathrm{T} 2=$ the day before surgery, $\mathrm{T} 3=$ the day of surgery, $\mathrm{T} 5=$ the day before the control visit, four weeks after surgery.

$\mathrm{M}=$ morning, $\mathrm{A}=$ afternoon, $\mathrm{E}$ evening, Pre = preoperative, Post = postoperative.

S1 Table. Saliva sampling times

\begin{tabular}{|c|c|c|c|c|c|c|c|c|c|c|c|c|}
\hline Sample & T1 M & $\mathrm{T} 1 \mathrm{~A}$ & $\mathrm{~T} 1 \mathrm{E}$ & T2 M & $\mathrm{T} 2 \mathrm{~A}$ & $\mathrm{~T} 2 \mathrm{E}$ & T3 M & T3 Pre & T3 Post & T5 M & T5 A & T5 E \\
\hline \multirow[t]{2}{*}{ Time } & 3:01 & $15: 22$ & $20: 36$ & $8: 02$ & $5: 2$ & $20: 37$ & $7: 15$ & 5 & 40 & $8: 1$ & $15: 27$ & $20: 50$ \\
\hline & :45) & $(1: 01)$ & $(0: 54)$ & D:59) & $(0: 48)$ & $(0: 35)$ & $(0: 55)$ & $(1: 42)$ & $(2: 15)$ & (1:09) & $(1: 12)$ & $(1: 2 \varepsilon$ \\
\hline
\end{tabular}

$\mathrm{T} 1$ = one week before surgery, $\mathrm{T} 2$ = the day before surgery, $\mathrm{T} 3$ = the day of surgery, $\mathrm{T} 5$ = the day before the control visit, four weeks after surgery.

$\mathrm{M}=$ morning, $\mathrm{A}=$ afternoon, $\mathrm{E}$ evening, Pre = preoperative, Post = postoperative. $\mathrm{hh}: \mathrm{mm}$, mean (SD). 
S1 File. Surgical Fear Questionnaire

Patient instructions:

This questionnaire assesses how afraid you are for various aspects related to the surgical procedure you are about to undergo. Please circle the number that best reflects how you feel right now.

1. I am afraid of the operation

2. I am afraid of the anesthesia

3. I am afraid of the pain after the operation

4. I am afraid of the unpleasant side effects (like nausea) after the operation

5. I am afraid my health will deteriorate because of the operation

6. I am afraid the operation will fail

7. I am afraid that I won't recover completely from the operation

8. I am afraid of the long duration of the rehabilitation after the operation

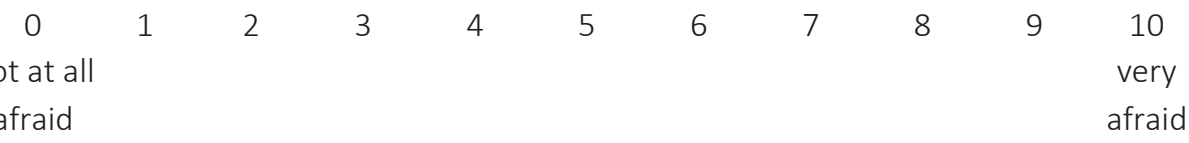

For the calculation of the total score the following instructions are applicable: one missing item score at maximum is allowed, to be replaced by the subject's mean score. In the case of more than one missing, the SFQ should not be interpreted. For the calculation of the subscales (short-term fear item 1-4 and long-term fear item 5-10) no missing data are allowed. If a subject enters two scores for one item: if adjacent, choose the highest value, if non-adjacent (other values are in between) the item has to be considered as missing. 



\title{
Chapter
}

\author{
Recovery 3 and 12 months after \\ hysterectomy: \\ epidemiology and predictors \\ of chronic pain, physical functioning, \\ and global surgical recovery
}

This chapter is published as: Maurice Theunissen, Madelon L. Peters, Jan Schepers, Jacques W.M. Maas, Fleur Tournois, Hans A. van Suijlekom, Hans-Fritz Gramke, Marco A.E. Marcus (2016). Recovery 3 and 12 months after hysterectomy: epidemiology and predictors of chronic pain, physical functioning, and global surgical recovery. Medicine (Baltimore). 95(26):e3980. 


\section{ABSTRACT}

Background: Chronic postsurgical pain (CPSP) is 1 important aspect of surgical recovery. To improve perioperative care and postoperative recovery knowledge on predictors of impaired recovery is essential. The aim of this study is to assess predictors and epidemiological data of CPSP, physical functioning (SF-36PF, 0-100), and global surgical recovery (global surgical recovery index, 0-100\%) 3 and 12 months after hysterectomy for benign indication.

Methods: A prospective multicenter cohort study was performed. Sociodemographic, somatic, and psychosocial data were assessed in the week before surgery, postoperatively up to day 4, and at 3- and 12-month follow-up. Generalized linear model (CPSP) and linear mixed model analyses (SF-36PF and global surgical recovery index) were used. Baseline data of 468 patients were collected, 412 (88\%) patients provided data for 3-month evaluation and 376 (80\%) patients for 12-month evaluation.

Results: After 3 and 12 months, prevalence of CPSP (numeric rating scale $\geq 4$, scale 0-10) was $10.2 \%$ and 9.0\%, respectively, SF-36PF means (SD) were 83.5 (20.0) and 85.9 (20.2), global surgical recovery index $88.1 \%$ (15.6) and $93.3 \%$ (13.4). Neuropathic pain was reported by 20 (5.0\%) patients at 3 months and 14 (3.9\%) patients at 12 months. Preoperative pain, surgery-related worries, acute postsurgical pain on day 4 , and surgeryrelated infection were significant predictors of CPSP. Baseline level, participating center, general psychological robustness, indication, acute postsurgical pain, and surgeryrelated infection were significant predictors of SF-36PF. Predictors of global surgical recovery were baseline expectations, surgery-related worries, American Society of Anesthesiologists classification, type of anesthesia, acute postsurgical pain, and surgeryrelated infection.

Discussion: Several predictors were identified for CPSP, physical functioning, and global surgical recovery. Some of the identified factors are modifiable and optimization of patients' preoperative pain status and psychological condition as well as reduction of acute postsurgical pain and surgery-related infection may lead to improvement of outcome. 


\section{ABBREVIATIONS}

AIC

ASA

BPI-SF

CES-D

CPSP

CzE

DN4-interview

GA

IQR

LAVH

$\mathrm{LH}$

LOT-R

Maastricht UMC+

MMC

MOS-SSS

NPP

NRS

OMC

PACU

PCS

SD

SF-36 PF

SFQ

W-BQ12
Akaike Information Criterion

American Society of Anesthesiologists

Brief Pain Inventory-Short Form

Center for Epidemiological Studies-Depression

chronic postsurgical pain

Catharina Hospital

Douleur neuropathique 4, self-reported items

general anesthesia

interquartile range, 25th to 75th percentile

laparoscopic assisted vaginal hysterectomy

laparoscopic hysterectomy

Life Orientation Test-Revised

Maastricht University Medical Center+

Máxima Medical Center

Medical Outcomes Study social support survey

neuropathic pain

numeric rating scale

Orbis Medical Center

postanesthesia care unit

Pain Catastrophizing Scale

standard deviation

RAND health survey short-form 36, physical functioning

Surgical Fear Questionnaire

Well-Being Questionnaire. 


\section{BACKGROUND}

In 2010, 11,697 hysterectomies were performed in women between 20 and 65 years old in the Netherlands. ${ }^{1}$ The prevalence of chronic postsurgical pain (CPSP) after hysterectomy is estimated between $5 \%$ to $32 \%{ }^{2}$ or even $50 \%{ }^{3}$; however, reported numbers are highly influenced by different definitions used for CPSP. But nonetheless, impaired recovery after hysterectomy may have huge social and economic impact. ${ }^{4-6}$ Therefore, further knowledge on incidence and risk factors of CPSP after hysterectomy is needed.

CPSP is just 1 aspect of postoperative recovery. Several other aspects may be important for a patient's experience of the success of an operation. Despite many publications on the concept and domains of postoperative recovery, there is no consensus yet about a generally accepted and validated assessment package for the evaluation of postoperative recovery. ${ }^{7}$ A study among hysterectomy patients, reflecting on their own recovery, revealed 4 domains: physical symptoms, emotional well-being, activity levels, and decision making. ${ }^{8}$ Other researchers described 5 domains of recovery, based on a concept analysis and interview studies among surgery patients and healthcare workers: physical symptoms, physical functions, psychological, social, and activity. ${ }^{9-12}$ This article will be based on a framework presented in 2013, proposing core predictor and outcome domains for CPSP in epidemiological studies, according to the IMMPACT recommendations. ${ }^{13,14}$ Four outcome domains are suggested: pain, physical functioning, psychological functioning, and global ratings of outcome. Within each domain outcome measures are suggested.

To improve perioperative care and postoperative recovery, knowledge on risk factors of impaired recovery is essential. Currently, many studies have provided insight into predictors and processes involved in chronic pain ${ }^{15}$ and other areas of postoperative recovery. ${ }^{16,17}$ One of the existing studies on this subject that assessed a subset of the whole range of potential predictors was performed in 2007 at the Maastricht University Medical Center+ (Maastricht UMC+). Predictors of CPSP, functional limitations, global surgical recovery, and quality of life 6 months after various types of surgery were assessed. ${ }^{18}$ The most important somatic predictors of suboptimal recovery were a long duration of the operation and high levels of acute postoperative pain. Psychological variables associated with suboptimal long-term outcome were a high level of preoperative fear of surgery and low optimism. However, despite the fact that studies like this can be very indicative, heterogeneity in types of surgery implies the need for further studies to be performed in homogeneous populations. Even in the case of 1 type of surgery under study, heterogeneity in study design may impair the strength of conclusions. ${ }^{2}$ To further refine our previous findings in a more homogeneous surgical population, and according to the call for studies on hysterectomy with detailed baseline and follow-up assessments ${ }^{4}$, the present prospective multicenter study was performed.

To obtain a broad view of the patients' preoperative status, a comprehensive set of demographic, medical, and psychosocial predictor variables was assessed. Of the out- 
come domains previously mentioned, that is, pain, physical functioning, global surgical recovery, and psychological recovery, this publication will focus on predictors for the first 3 domains. The selection of predictor variables was based on findings from previous studies on CPSP and supplemented with some general background characteristics of patients and routinely obtained medical variables. ${ }^{2,15,18-21}$ Important candidate predictors were acute postoperative pain, preoperative pain, and several psychosocial variables. For pre- and postoperative pain, besides the intensity, also location, duration, onset, interference, and the neuropathic character were assessed. The psychosocial variables included both vulnerability and resilience factors. Previous studies have shown that anxiety and pain catastrophizing are among the most important vulnerability factors for CPSP. ${ }^{22}$ Other variables that have previously been associated with higher prevalence of CPSP are depression ${ }^{23,24}$ and negative outcome expectancies. ${ }^{25,26}$ We also included a measure of childhood abuse as this has been related to the prevalence of chronic pain in general ${ }^{27}$ and more specifically to gynecological pain. ${ }^{28}$ One of the most prominent resilience factors for CPSP appears to be optimism. ${ }^{29-31}$ In addition, social support $^{32}$ and more general measures of wellbeing ${ }^{30,33}$ have been proposed as protective factors. Thus, a comprehensive set of demographic, medical, and psychosocial variables was obtained and assessed for its association with long-term surgical outcome.

The primary aim of this study was to establish somatic and psychosocial risk factors for pain after 3 and 12 months, in Dutch patients undergoing elective hysterectomy. Secondary aims were to establish somatic and psychosocial risk factors for poor physical functioning and global surgical recovery, to assess the prevalence, incidence, and characteristics of chronic pain, and to assess epidemiological data regarding the secondary outcome variables and predictors. Epidemiological data on predictors and outcomes on the 3 domains of recovery will be described for the total population, and those concerning the predictors also separately for patients with and without CPSP. Also, patientreported complications during follow-up will be described. To provide better insight in the course of recovery, outcome assessment was performed at multiple relevant time points: postoperative day 1 to 4 , and at 3 and 12 months following surgery.

\section{METHODS}

For this study, approval of the local Medical Ethical Committee's was obtained and all participants gave informed consent. This study was registered at the Dutch Trial Register under number NTR2702 (http://www.trialregister.nl/trialreg/index.asp). This article was written in accordance with the STROBE guidelines. ${ }^{34}$

A prospective multicenter cohort study was performed in 4 hospitals in the Netherlands: the Maastricht UMC+ Maastricht, the Catharina Hospital (CzE) Eindhoven, the Máxima Medical Center (MMC) Veldhoven, and from May 2012, the Orbis Medical 
Center (OMC) Sittard-Geleen. Surgery was performed between September 2010 and January 2014. Patients were admitted to the gynecology or the short stay ward. Data collection took place in the week before surgery, postoperatively up to day 4, and at 3and 12-month follow-up. Preoperative and follow-up assessments were performed at home by postal questionnaires. Pain was recorded in a pain diary, provided by the study coordinator, until 4 days after the operation. In case of earlier discharge, the diary was continued at home. Pain medication was recorded during the hospital stay only and stopped with discharge. Data on surgery and anesthesia were collected during surgery by the attending anesthesiologist, gynecologist, or study coordinator.

Participants underwent hysterectomy for a benign indication in 1 of the hospitals involved in the study. Patients were selected for inclusion during the preoperative screening visit. Inclusion criteria were informed consent, age between 18 and 65 years, good command of the Dutch language, elective surgery, and total or subtotal hysterectomy with or without oophorectomy. All types and combinations of surgical approach were allowed: vaginal, abdominal, or laparoscopic. Exclusion criteria were history of cancer, illiteracy, and cognitive impairment, as indicated in the medical record or assessed during the informed consent procedure. Patients with a history of malignancy or an oncological indication for surgery were excluded because of potential differences in prognosis as well as perioperative treatment like chemotherapy or radiotherapy that might bias preoperative baseline and predictor data as well as postoperative outcome.

\section{Confounding and data management}

Confounding was addressed in several ways. A homogeneous population was selected, that is, females between 18 and 65 years, only hysterectomy as type of surgery, and absence of malignancy. Data were collected prospectively, missing perioperative data were extracted afterward from the medical file, when available. If the pain diary or follow-up questionnaire was not returned, patients received 1 reminder by post. Missing values on multi-item psychosocial questionnaires, physical functioning, and pain interference scale, if $<20 \%$, were imputed by the participants' mean score on that scale. Single-item sociodemographic, surgical, and outcome variables were not imputed. Use was made of validated questionnaires. All questionnaire packages were designed in collaboration with MEMIC Maastricht, center for data and information management. Completed questionnaires were scanned by MEMIC Maastricht and provided directly in SPSS format. Statistically, using multivariate analyses, the collected control and process variables allowed for correction of bias caused by baseline status of outcome and sociodemographic or surgical factors. Finally, all patients who underwent other surgery or reported a malignancy during follow-up were excluded from follow-up analysis. 


\section{Baseline and predictor measures}

Baseline data consisted of the sociodemographic variables such as age, education level, employment status, marital status, number of children, and gynecological history, including whether women were sexually active or not. Health behavior (smoking and number of active days/week, minimum of 30 min of physical activity), general health status, and comorbidity were assessed using the screening list developed by Statistics Netherlands. ${ }^{35}$ American Society of Anesthesiologists (ASA) physical status classification was recorded. Physical functioning was measured with the physical functioning subscale of the RAND health survey short-form 36 (SF-36). This subscale consists of 10 items assessing perceived difficulties in physical activities, range from 0 , indicating severely restricted physical activity, to 100 indicating unrestricted physical activity. ${ }^{36,37}$

Pre-existing pain was assessed by the Brief Pain Inventory-Short Form (BPI-SF), including location, duration, intensity, intermittence, and pain therapy. The BPI-SF is a 12item self-administered questionnaire which references pain during the past $24 \mathrm{~h}$. It consists of 2 subscales: pain severity (4 items) and pain interference (7 items). Questionnaire items are scaled from 0 (meaning no pain/no interference) to 10 (meaning worst pain/complete interference). ${ }^{38}$ The scale was slightly adapted to assess intensity of both hysterectomy-related (i.e., pain related to the planned hysterectomy and gynecological pain) and nonhysterectomy-related pain over the last week instead of the last $24 \mathrm{~h}$. We added 1 item to assess "any pain" at the moment of completion of the questionnaire, using the numeric rating scale (NRS) 0 to 10. In order to further characterize the pain, the Douleur neuropathique 4 (DN4) was administered. The DN4 is a 10-item screening instrument to assess the neuropathic character of the pain. Because examination at home by study team members was not possible, only the 7 self-reported items on neuropathic pain (NPP) could be applied, the DN4-interview. When using this 7-item variant of the instrument, a score $\geq 3$ indicates presence of NPP, with a sensitivity of $78 \%$ and specificity of $81 \%{ }^{39}$ In case of missing data on the DN4-interview scale, NPP was considered present if at least 3 positive scores were completed on the DN4-interview scale, or absent in the case of at least 5 negative scores.

Collected surgery-related data were type of incision (median lower abdominal, Pfannenstiel, vaginal, laparoscopic hysterectomy [LH], or laparoscopic assisted vaginal hysterectomy [LAVH]), total or subtotal hysterectomy with or without oophorectomy, indication for the operation, type of anesthesia (general [GA], spinal, GA combined with epidural or spinal), postoperative analgesics, duration of operation, blood loss, complications, conversion of incision type, hospital where the operation took place, and experience of the attending gynecologist expressed as years of training including fellowship. Acute postsurgical pain was assessed until day 4. Every evening patients reported pain at rest and at movement, highest and average pain over the last 24h (NRS 0-10), use of pain medication yes/no, and at day 4 also NPP. 
The psychological predictors included were outcome expectancy, surgical fear, pain catastrophizing, optimism, social support, depression, well-being, and childhood abuse. Outcome expectancies were measured on the basis of 3 items: expected pain intensity at postoperative day $4,^{25}$ expected level of surgical recovery 3 months after hysterectomy assessed with the global surgical recovery index, ${ }^{40}$ and expected time until full return to work/normal activities. ${ }^{25}$ The first 2 items were chosen so as to match the corresponding outcome domains assessed in this study. As previously noted, expectancy measures whose domain of behavior matches that of the outcome will have the highest predictive value. ${ }^{41,42}$ In addition, expectations about the effect of hysterectomy on feelings of femininity and whether hysterectomy would mean a relief or a loss ${ }^{43,44}$ were assessed on the basis of 2 tailored items. Surgical fear was measured by using the 8-item Surgical Fear Questionnaire (SFQ), whose validation was recently described. ${ }^{45}$ Pain catastrophizing was measured with the Dutch version of the Pain Catastrophizing Scale (PCS). The PCS consists of 13 items assessing an exaggerated negative interpretation of the meaning of pain. It has good reliability and validity. ${ }^{46}$

Optimism/pessimism was assessed by the revised Life Orientation Test (LOT-R). The LOT-R has 10 items, 3 of which measure a positive outlook on the future and 3 items measure a negative outlook. After reversal of the negative items, a single-optimism score can be obtained. ${ }^{47}$ Social support was measured with the Dutch version of the Medical Outcomes Study-social support survey (MOS-SSS). ${ }^{48}$ This is a 19-item self-report questionnaire with 4 support subscales: emotional/informational, tangible, positive interaction, and affectionate. We present the overall support index and the number of close friends/relatives available for support. Depression was measured with the Center for Epidemiological Studies-Depression (CES-D) questionnaire. ${ }^{49}$ This self-report instrument is developed for the assessment of depressive symptoms in the general population. Psychological well-being was assessed with the 12-Item Well-Being Questionnaire (W-BQ12) ${ }^{50}$ The W-BQ12 provides a brief measure of positive well-being, energy and negative well-being. The W-BQ12 avoids the use of somatic items, and is therefore particularly suitable for use in patient populations. The negative well-being subscale of W-BQ12 was omitted to avoid overlap with the CES-D. Childhood physical or sexual abuse was assessed using a validated 2 -item screening questionnaire. ${ }^{51}$

\section{Outcome measures}

Predictors were assessed for 3 outcomes of postoperative recovery at 3- and 12-month follow-up. For the primary outcome, CPSP, the BPI-SF was used. Patients first had to answer a question about whether they had pain related to the surgical procedure or not. If so, the BPI-SF had to be completed. In the case of absence of hysterectomyrelated pain, they could continue with the next section of the questionnaire and the pain scores were replaced by 0 . Predictor analysis was performed with 1 of the 4 pain severity items, the highest hysterectomy-related pain score during the last week, NRS 0 
to 10. In accordance with clinical practice and previous CPSP studies, the NRS score was dichotomized into the no-CPSP group, NRS 0 to 3 indicating no or slight pain, and the CPSP group, NRS 4 to 10 indicating moderate to severe pain. ${ }^{52,53}$ The second outcome measure used for predictor analysis was SF-36 physical functioning, range 0 to 100 . The third outcome measure in predictor analysis was self-perceived recovery, assessed with the global surgical recovery index. The global surgical recovery index is a generic 1-item scale on which patients score to what extent they feel recovered from surgery. The patients themselves decide which aspects of recovery are most relevant and thus mirrored in their recovery score. The scale ranges from $0 \%$, meaning not recovered at all, to $100 \%$, meaning full recovery. It has been used in previous surgical studies and the correlation with another, more extensive, recovery scale was $0.72 .^{18,40}$

In line with the secondary aim, epidemiological data on outcome measures and predictors are presented. For numbers of prevalence and incidence, the scores were dichotomized if applicable. The primary outcome measure pain was dichotomized into the no-CPSP group, NRS from 0 to 3, and the CPSP group, NRS 4 to 10, based on NRS highest hysterectomy-related pain score. In addition to the primary outcome, the neuropathic characters of the pain (DN4-interview), location, onset, and intermittence of the hysterectomy-related pain were assessed. Intensity and location of nonhysterectomy-related pain was also assessed. Patients were asked to report on pain interference, analgesic use, and other forms of pain treatment. Furthermore, health behavior was assessed using the overall physical activity question from the screening list developed by Statistics Netherlands: "How many days per week do you have physical exercise during at least 30 min ?" ${ }^{\prime 35}$ Also, 1 question assessed the number of days after which the patient was capable of performing all activities of daily living again. Finally, the incidence of surgery-related infections, healthcare visits as a result of a complication during follow-up, ${ }^{54}$ and the incidence of significant health events, such as an accident, were explored by 3 questions. Data on sexuality, baseline genetics, and psychological recovery were also collected, but to avoid overload of data these results will be published separately at a later stage.

\section{Statistical analyses}

We planned to include 500 patients. With an expected loss of $40 \%$ (due to either dropout or exclusion due to reoperations and new malignancies), 300 patients were expected to be available for the follow-up analyses. With a regression analysis with 7 prior covariates (control variables) explaining $20 \%$ of variance, alpha set at 0.05 and a power of $90 \%$, this would enable the detection of an R-square increase of $0.027 \quad(R=0.16)$. Eventually 517 participants were included, 412 were available for the analyses at 3month follow-up and 376 at 12-month follow-up.

Data were visually assessed for normality by creating histograms and by ShaphiroWilk test. Pain intensity and interference scores are presented as median and interquar- 
tile range $\left(25^{\text {th }}-75\right.$ th percentile, IQR) and analyzed using Mann-Whitney $U$ test because of non-normal distribution. Other continuous data were tested by Student $t$ test and presented as mean and standard deviation (SD). Categorical data are presented as number (\%) and analyzed using Chi-squared or Fischer exact test. Baseline and followup results are shown for the total study sample. Baseline data and self-reported complications are also shown for the groups CPSP and no CPSP separately, for both 3- and 12month outcomes, available as Supplementary File (Tables 1B, 2B, 3B, 4B, and 6, http://links.lww.com/MD/B57).

Predictor analysis of the primary outcome pain at 3- and 12-month follow-up was performed on the highest hysterectomy-related pain score during the last week, the noCPSP group versus the CPSP group. Because of the dichotomized outcome CPSP, generalized linear model analysis was performed using binary logistic regression and furthermore a time variable defining 3- and 12-month follow-up. The results are presented as beta (SD). See Supplementary Table 5B, http://links.Iww.com/MD/B57 "Final multivariate models: additional legend concerning data reduction and analysis" for detailed information on the statistical analysis. Predictor analyses for the SF-36 physical functioning and global surgical recovery index scores were performed on continuous scores. No clinically accepted cut-off point is available for global surgical recovery index, while the SF-36 physical functioning score can only be interpreted in light of the presurgical SF-36 score. For these outcomes, linear-mixed model analysis with random intercept and a time variable defining 3- and 12-month follow-up was used. Results are presented as estimate (SD), which reflect the change in outcome for each unit of change in the predictor. Goodness-of-fit model is reflected by Akaike Information Criterion (AIC), the lower the better the fit.

To reduce the large number of available predictor variables that could potentially be used in the regression analyses, first bivariate association with the 3 outcomes was assessed. Predictor variables were only entered in the multivariate model if significant at $p<0.10$ level in bivariate analysis. Furthermore, to maintain a certain uniformity across multivariate analyses of the 3 outcome measures and to further reduce the number of variables, for predictors with a high level of similarity or overlap an a priori choice was made regarding which 1 to retain. This selection was based on congruency between predictor and outcome measures, general applicability or frequent clinical use, or contribution to model fit. See Supplementary Table 5B, http://links.lww.com/MD/B57 "Final multivariate models: additional legend concerning data reduction and analysis" for detailed information on the process of data reduction. In the multivariate analyses, type of incision was dichotomized as laparotomy versus vaginal/LH/LAVH. Type of anesthesia was dichotomized as GA versus other, including combination of types. Finally, to reduce the number of psychological predictors a factor analysis with oblimin rotation was performed on the SFQ, PCS, LOT-R, CES-D, and W-BQ12. Principal component analysis revealed 2 factors. The first factor consisted of the SFQ and PCS and was named surgeryrelated worries. Thus, this factor represents high fear of surgery and high-pain catastro- 
phizing. The second factor consisted of LOT-R, CES-D, and W-BQ12 and was named general psychological robustness. This factor represents a high level of optimism and psychological well-being and a low level of depressive symptoms. Aggregate scores were calculated by summing the weighted scores on the respective questionnaires belonging to the 2 new variables. The MOS-SSS was analyzed separately because, in contrast to the selected 5 psychological variables that reflect intraindividual differences, the MOS-SSS reflects interindividual differences, namely the availability of social support. Also, childhood physical or sexual abuse and outcome expectancies were entered as separate predictors. The outcome expectancy variable corresponding with the particular outcome was used in the multivariate analysis. For the outcome CPSP this was expected pain intensity, and for outcome global surgical recovery this was expected global surgical recovery. For outcome physical functioning, no corresponding baseline expectation was assessed. Expectancies of hysterectomy as a relief or loss and expectancy regarding femininity were used for all outcomes.

The control variables, such as hospital, age, type of incision (dichotomized), type of anesthesia (dichotomized), and the time variable defining 3- and 12-month follow-up, were entered in the multivariate model using a forced entry method. All other predictors were entered using a backward deletion procedure (criterion $p<0.10$ ). A final significance level of $p<0.05$ was used. For each outcome, 2 prediction models are presented. Model 1 reveals the results of multivariate prediction analyses using the control variables supplemented with the significant preoperative predictors. This allows establishing a preoperative risk profile. In model 2, the final model, the pre- and postoperative predictors are added. For all 3 outcome measures, multicollinearity was assessed using the variance inflation factor and tolerance, obtained by entering the predictors used in the multivariate models in a linear regression model. Criteria for absence of multicollinearity are variance inflation factor $<10$ and average variance inflation factor around 1, tolerance $\geq 0.2$. Analyses were performed using IBM SPSS Statistics for Windows, Version 22.0 (Armonk, NY).

\section{RESULTS}

Patient inclusion is illustrated in the flow chart in Figure 1. Of the 517 patients providing informed consent, baseline data were obtained from 468 patients. The response rate after 3 months was high with 412 (88\%) of 468 operated patients suitable for follow-up evaluation. After 12 months, 376 (80\%) patients were suitable for follow-up evaluation. The number of missing data was relatively low: for baseline data range $0 \%$ to $12.9 \%$, surgery data $0 \%$ to $5.1 \%$, acute pain data $11.2 \%$ to $17.3 \%$, and follow-up data $0 \%$ to $5.6 \%$. Sociodemographic and baseline health characteristics are presented in Table 1 and 1B (Supplementary File, baseline data split up for the groups CPSP and no CPSP, http://links.Iww.com/MD/B57). Psychosocial baseline measures and data concerning 
surgery and acute postsurgical pain are presented in Tables 2 and 3 (Tables 2B and 3B, Supplementary File, http://links.Iww.com/MD/B57). Most commonly applied interventions were vaginal hysterectomy and GA. Preoperative pain characteristics and other physical baseline parameters are presented in Table 4, together with their corresponding outcomes after 3 and 12 months. Similarly to the other baseline characteristics, in Table 4B (Supplementary File, http://links.Iww.com/MD/B57), baseline data on pain and physical functioning are presented split up for the groups CPSP and no CPSP at 3 and 12 months, respectively.

\section{Descriptive data of recovery variables}

CPSP at 3-month follow-up (hysterectomy-related pain, NRS highest pain last week, cutoff $\geq 4$ ) was indicated by 42 patients (the CPSP group), prevalence $10.2 \%$, 9 of whom had no pain at baseline, incidence $2.2 \%$. In 169 (82.8\%) of the patients reporting preoperative hysterectomy-related pain, the pain had disappeared at 3-month follow-up. Median (IQR) pain intensity in the CPSP group was 6 (4.8-7.3) and in the no-CPSP group 0 (0-0) (hysterectomy-related NRS highest pain last week). Nonhysterectomy-related pain at 3 months (NRS highest pain last week, cut-off $\geq 4$ ) was indicated by 102 (25.0\%) women. Any pain at 3-month follow-up (hysterectomy- and/or nonhysterectomy-related pain at the moment of completing the questionnaire, NRS cut-off $\geq 4$ ) was indicated by 67 $(17.0 \%)$ women. An indication of NPP (DN4-interview $\geq 3$ ) was present in 16 patients (45.7\%) of the CPSP group versus 4 patients (1.1\%) of the no-CPSP group. Occurrence of NPP in relation to type of incision was median lower abdominal $0(0 \%)$, Pfannenstiel 6 (14.0\%), vaginal 6 (3.7\%), LH 3 (3.7\%), and LAVH 5 (5.7\%) cases. NPP occurred in 19 patients $(5.7 \%)$ with GA and in 1 (2.0\%) with spinal analgesia. Three months after surgery, $61.9 \%$ of the patients in the CPSP group used pain medication as compared with $24.4 \%$ of the patients of the no-CPSP group. Pain interference in the CPSP group was 3.9 (2.9-5.6).

At 12 months a minor decrease of CPSP was shown, with 34 women reporting an NRS $\geq 4$, prevalence 9.1\%. Pain intensity (hysterectomy-related pain, NRS highest pain last week) in the CPSP group was 6 (5-7) and in the no-CPSP group 0 (0-0). Nonhysterectomy-related pain was reported by 121 patients (32.5\%), an increase compared with the 3-month results. Any pain at 12-month follow-up (hysterectomy- and/or nonhysterectomy-related pain at the moment of completing the questionnaire) was indicated by 70 (19.7\%) women. Hysterectomy-related NPP was reported by 10 women (38.5\%) of the CPSP group and 4 women (1.2\%) of the no-CPSP group. In the CPSP group, 19 women used analgesia (55.9\%) versus 95 (28.4\%) of the women in the no-CPSP group. Pain interference in the CPSP group was 3.1 (2.1-6.6). 

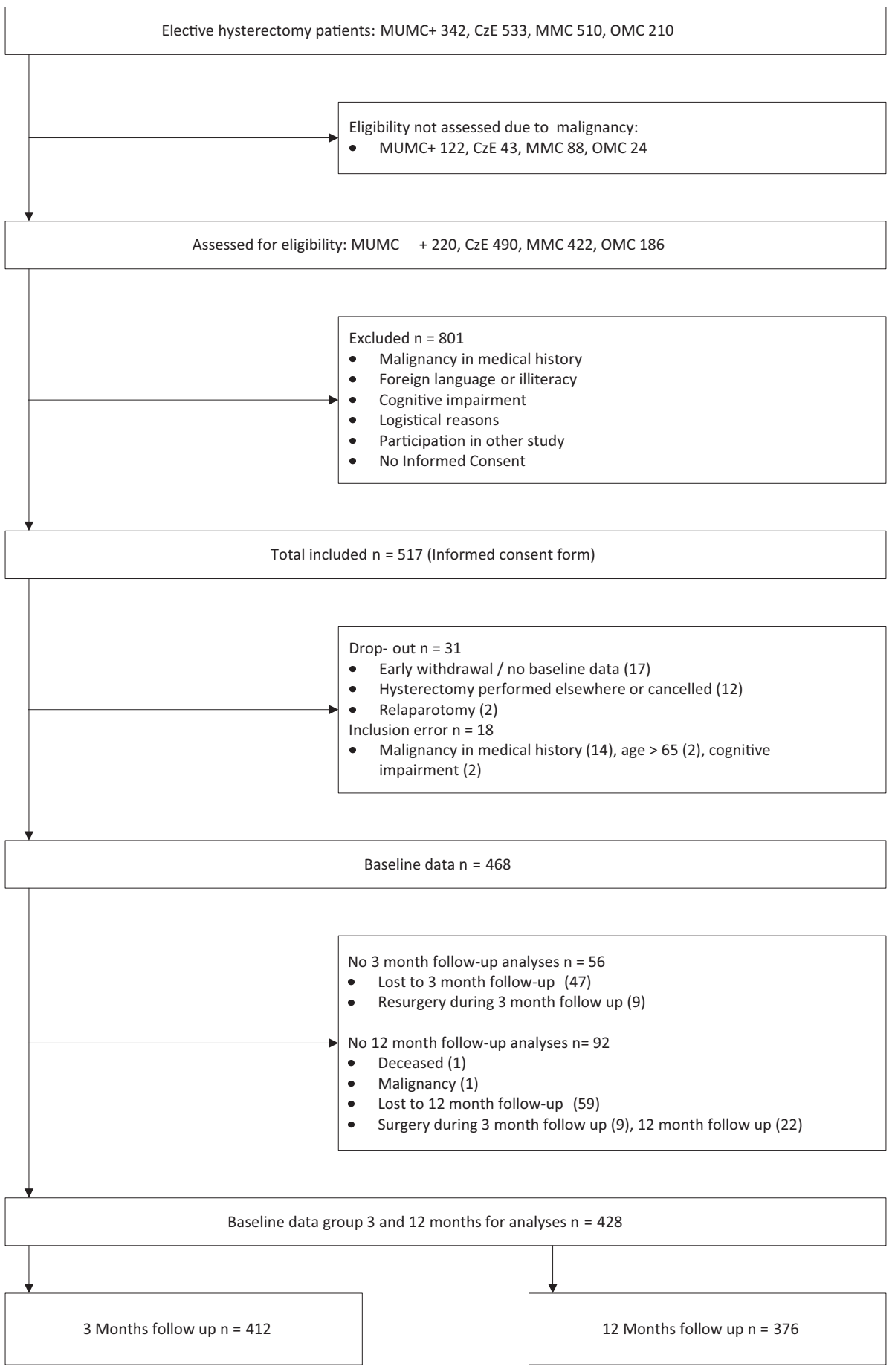

Figure 1. Flow chart patient inclusion 


\section{Chapter 5}

Table 1. Baseline characteristics

\begin{tabular}{|c|c|}
\hline \multirow[t]{2}{*}{ Baseline measures } & Baseline sample \\
\hline & $N=428$ \\
\hline \multicolumn{2}{|l|}{ Center } \\
\hline Maastricht UMC+ & 121 \\
\hline $\mathrm{CzE}$ & 154 \\
\hline MMC & 115 \\
\hline OMC & 38 \\
\hline Age & $46.9(7.1)$ \\
\hline Native country: the Netherlands & $401(94 \%)[1]$ \\
\hline Marital status: living together & $363(85 \%)[2]$ \\
\hline Employment: paid job & $308(72 \%)[2]$ \\
\hline \multicolumn{2}{|l|}{ Education } \\
\hline No / lower & $65(15 \%)$ \\
\hline Intermediate & $249(58 \%)$ \\
\hline University / higher & $113(27 \%)$ \\
\hline Missing data & 1 \\
\hline \multicolumn{2}{|l|}{ General health } \\
\hline Very poor / poor & $5(1 \%)$ \\
\hline Moderate & 77 (18\%) \\
\hline Very good / good & $345(81 \%)$ \\
\hline Missing data & 1 \\
\hline \multicolumn{2}{|l|}{ Smoking } \\
\hline Yes & $97(23 \%)$ \\
\hline No & $170(40 \%)$ \\
\hline Stopped & $156(37 \%)$ \\
\hline Missing data & 5 \\
\hline \multicolumn{2}{|l|}{ ASA I / II / III } \\
\hline 1 & $234(57 \%)$ \\
\hline$\|$ & $169(41 \%)$ \\
\hline III & $7(2 \%)$ \\
\hline Missing data & 18 \\
\hline Body mass index & $26.5(4.9)$ \\
\hline \multicolumn{2}{|l|}{ Medical history } \\
\hline Chronic disease & $152(36 \%)$ \\
\hline Psychological problems & $122(29 \%)[3]$ \\
\hline Surgery last year & $113(27 \%)[5]$ \\
\hline Hysterectomy-related surgery last year & $75(18 \%)[4]$ \\
\hline \multicolumn{2}{|l|}{ Gynecological history } \\
\hline Premenopausal state & $322(77 \%)[8]$ \\
\hline Hormonal replacement therapy & $15(4 \%)[3]$ \\
\hline Contraception use & $88(21 \%))[3]$ \\
\hline Gravidity & $353(84 \%)[8]$ \\
\hline Parity & $1.8(1.1)$ \\
\hline Vaginal delivery or caesarean section & $351(83 \%)[6]$ \\
\hline Caesarean section & $61(14 \%)[2]$ \\
\hline Sexually active & $341(83 \%)[17]$ \\
\hline \multicolumn{2}{|l|}{ Indication (more than 1 possible) } \\
\hline Leiomyoma & 139 \\
\hline Prolapse & 74 \\
\hline
\end{tabular}




\begin{tabular}{ll}
\hline Menorrhagia / metrorrhagia & 207 \\
Dysmenorrhoea & 27 \\
Endometriosis / adenomyosis & 23 \\
Abdominal pain & 26 \\
Cervical dysplasia & 24 \\
Other & 25 \\
\hline
\end{tabular}

Numbers are mean (standard deviation) or number (\%) [missing data].

Medical history, psychological problems: answer yes to "Did you ever suffer from psychological problems for which you needed support?"

$\mathrm{ASA}=$ American Society of Anesthesiologists, $\mathrm{CzE}=$ Catharina Hospital Eindhoven, Maastricht UMC+=Maastricht University Medical Center+, MMC=Máxima Medical Center, Veldhoven, OMC=Orbis Medical Center, SittardGeleen.

Table 2. Psychosocial baseline measures

\begin{tabular}{|c|c|}
\hline Baseline measures & $\begin{array}{l}\text { Baseline sample } \\
\mathrm{N}=428\end{array}$ \\
\hline Expected pain after 4 days (0-10) & $5.0(3-6)$ \\
\hline Expected pain after 4 days NRS $\geq 4$ & $308(74 \%)[10]$ \\
\hline Expected GSR \% after 3 months & $93.0(11.3)$ \\
\hline Expected number of days until full activities & $44(31)$ \\
\hline \multicolumn{2}{|l|}{ Expectation hysterectomy: } \\
\hline Relief & $319(76 \%)$ \\
\hline Neutral & $92(22 \%)$ \\
\hline Loss & $10(2 \%)$ \\
\hline Missing data & 7 \\
\hline \multicolumn{2}{|l|}{ Expectation less femininity: } \\
\hline Not at all / a bit less & $413(98 \%)$ \\
\hline Rather much less / very much less & $10(2 \%)$ \\
\hline Missing data & 5 \\
\hline Surgical fear short term (0-40) & $16.0(9.9)$ \\
\hline Surgical fear long term (0-40) & $9.3(8.6)$ \\
\hline PCS (0-52) & $12.2(8.3)$ \\
\hline LOT-R (0-24) & $17.0(3.7)$ \\
\hline MOS-SSS number of persons & $9.9(6.9)$ \\
\hline MOS-SSS total (0-100) & $85.7(18.5)$ \\
\hline CES-D $(0-60)$ & $10.9(8.3)$ \\
\hline WBQ energy (0-12) & $7.0(2.7)$ \\
\hline WBQ positive well-being (0-12) & $7.6(3.0)$ \\
\hline History of physical abuse & $51(12 \%)[3]$ \\
\hline History of sexual abuse & $83(20 \%)[7]$ \\
\hline
\end{tabular}

Expected pain data are given as median (interquartile range), all others as mean (standard deviation) or number (\%) [missing data].

CES-D=Center for Epidemiological Studies-Depression, GSR \%=global surgical recovery index, 0-100\%, LOT$\mathrm{R}=$ life orientation test-revised, MOS-SSS=Medical Outcomes Study-social support survey with number of close friends/relatives and total score of the subscales tangible support, emotional/informative support, affective support, and positive social interaction, NRS=numeric rating scale, $P C S=$ pain catastrophizing scale, WBQ=WellBeing Questionnaire, energy and positive wellbeing subscales. 


\section{Chapter 5}

Table 3. Surgery and acute pain

\begin{tabular}{|c|c|}
\hline \multirow[t]{2}{*}{ Measures } & \multirow{2}{*}{$\begin{array}{l}\text { Baseline sample } \\
\mathrm{N}=428\end{array}$} \\
\hline & \\
\hline \multicolumn{2}{|l|}{ Anesthesia } \\
\hline GA & $358(84 \%)$ \\
\hline GA and epidural & $14(3 \%)$ \\
\hline spinal & $52(12 \%)$ \\
\hline GA and spinal & $4(1 \%)$ \\
\hline duration (hours) & $2: 17(0: 53)$ \\
\hline \multicolumn{2}{|l|}{ Hysterectomy } \\
\hline Total $^{+}$ & $402(95 \%)[3]$ \\
\hline Oophorectomy & $33(8 \%)$ \\
\hline Prolapse surgery & $78(19 \%)[22]$ \\
\hline \multicolumn{2}{|l|}{ Incision } \\
\hline Median lower abdominal & $23(5 \%)$ \\
\hline Pfannenstiel & $51(12 \%)$ \\
\hline Vaginal & $175(41 \%)$ \\
\hline $\mathrm{LH}$ & $87(20 \%)$ \\
\hline LAVH & $92(22 \%)$ \\
\hline Blood loss (ml) & $279(370)$ \\
\hline Training gynecologist ${ }^{\ddagger}$ (years) & $14.0(9.7)$ \\
\hline \multicolumn{2}{|l|}{ Pain PACU ${ }^{\S}$} \\
\hline At rest $(0-10)$ & $3.0(0.0-5.0)$ \\
\hline At rest $N R S \geq 4$ & $160(42 \%)[48]$ \\
\hline \multicolumn{2}{|l|}{ Pain postoperative day 4} \\
\hline Highest pain last 24 h $(0-10)$ & $3.0(1.0-5.0)$ \\
\hline Highest pain last $24 \mathrm{~h}$ NRS $\geq 4$ & $143(39 \%)[57]$ \\
\hline Average pain last $24 \mathrm{~h}(0-10)$ & $2.0(1.0-4.0)$ \\
\hline Average pain last $24 \mathrm{~h} N R S \geq 4$ & $96(26 \%)[54]$ \\
\hline $\mathrm{DN}-4^{\| \prime} \geq 3$ & $84(24 \%)[74]$ \\
\hline Using pain medication postoperative day 4 & $264(71 \%)[56]$ \\
\hline
\end{tabular}

Numbers are mean (standard deviation), median (interquartile range), or number (\%) [missing data].

DN4=neuropathic pain questionnaire, GA=general anesthesia, LAVH=laparoscopic assisted

vaginal hysterectomy, $\mathrm{LH}=$ laparoscopic hysterectomy, $\mathrm{NRS}=$ numeric rating scale, $\mathrm{PACU}=$

postanesthesia care unit.

+ Compared with subtotal hysterectomy.

¥ Years of experience including residentship of the 1st responsible gynecologist.

$\S$ Pain $1 \mathrm{~h}$ after arrival at PACU.

I| Seven self-report items (DN4-interview).

The mean scores on the SF-36 physical functioning subscale of 83.5 (SD 20.0) at 3month follow-up appeared to be relatively stable when compared with the preoperative scores of 82.4 (19.3). At 12 months the mean score was 85.9 (20.2). Self-perceived recovery after 3 months was high with a mean global surgical recovery index score of 
88.1 (15.6), but significantly lower than the expected global surgical recovery index of 93.2 (10.8) $(p<0.001)$. However, the expected level was achieved after 12 months of recovery with a mean score of 93.9 (SD 13.4). The time until patients were able to perform their normal daily activities, reported after 3 months, was 44 days. This was in line with their expectations. However, the same question asked at 12 months revealed a mean of 69 days. Data on CPSP, SF-36 physical activity, global surgical recovery, and other indicators of recovery are presented in Table 4. For the outcomes on pain intensity, pain interference, analgesia use, SF-36 physical activity, global surgical recovery, and proportion of women able to perform daily activities again, the scores of patients in the CPSP group are significantly less favorable than for patients in the no-CPSP group, at both 3 and 12 months.

Table 4. Baseline measures and corresponding recovery scores at 3 and 12 months after hysterectomy

\begin{tabular}{|c|c|c|c|}
\hline \multirow[t]{2}{*}{ Measures } & \multirow{2}{*}{$\begin{array}{l}\text { Baseline sample } \\
N=428\end{array}$} & \multirow{2}{*}{$\begin{array}{l}\text { Sample } \\
3 \text { month follow-up } \\
\mathrm{N}=412\end{array}$} & \multirow{2}{*}{$\begin{array}{l}\text { Sample } \\
12 \text { month follow-up } \\
N=376\end{array}$} \\
\hline & & & \\
\hline \multicolumn{4}{|l|}{ Pain } \\
\hline \multicolumn{4}{|l|}{ Hysterectomy-related pain intensity: } \\
\hline Highest pain last week $(0-10)$ & $3.5(0.0-7.0)$ & $0.0(0.0-0.0)$ & $0.0(0.0-0.0)$ \\
\hline Highest pain last week NRS $\geq 4$ & $214(50 \%)$ & $42(10 \%)[2]$ & $34(9 \%)[1]$ \\
\hline Average pain last week (0-10) & $2.0(0.0-5.0)$ & $0.0(0.0-0.0)$ & $0.0(0.0-0.0)$ \\
\hline Average pain last week NRS $\geq 4$ & $177(41 \%)$ & $33(8 \%)[2]$ & $26(7 \%)[2]$ \\
\hline \multicolumn{4}{|c|}{ Hysterectomy-related neuropathic pain: } \\
\hline $\mathrm{DN} 4^{+} \geq 3$ & 41 (10\%) [30] & $20(5 \%)[15]$ & 14 (4\%) [13] \\
\hline \multicolumn{4}{|l|}{ Hysterectomy-related pain location: } \\
\hline Lower abdomen & $246(58 \%)$ & $60(15 \%)$ & $40(11 \%)$ \\
\hline Upper abdomen & $23(5 \%)$ & $15(4 \%)$ & $3(1 \%)$ \\
\hline Back & $143(33 \%)$ & $19(5 \%)$ & $17(5 \%)$ \\
\hline Legs & $51(12 \%)$ & $7(2 \%)$ & $8(2 \%)$ \\
\hline Breast & $16(4 \%)$ & $5(1 \%)$ & $7(2 \%)$ \\
\hline Other location & $23(5 \%)$ & $13(3 \%)$ & $7(2 \%)$ \\
\hline \multicolumn{4}{|c|}{ Onset hysterectomy-related pain since: } \\
\hline 1 week & $6(2 \%)$ & $8(10 \%)$ & $1(2 \%)$ \\
\hline 1 month & $8(3 \%)$ & $6(8 \%)$ & $2(3 \%)$ \\
\hline 2 months & $23(9 \%)$ & $5(6 \%)$ & $8(14 \%)$ \\
\hline 3-9 months & NA & NA & $9(16 \%)$ \\
\hline 3-12 months & $69(26 \%)$ & NA & NA \\
\hline$>12$ months & 157 (60\%) & NA & NA \\
\hline hysterectomy & NA & $60(76 \%)$ & $37(65 \%)$ \\
\hline NA & 165 & 333 & 319 \\
\hline \multicolumn{4}{|c|}{ Intermittence hysterectomy-related pain: } \\
\hline Brief/short & $19(7 \%)$ & $24(32 \%)$ & $13(23 \%)$ \\
\hline Periodic/recurrent & $173(67 \%)$ & $38(50 \%)$ & $32(56 \%)$ \\
\hline
\end{tabular}


Chapter 5

\begin{tabular}{|c|c|c|c|}
\hline \multirow[t]{2}{*}{ Measures } & Baseline sample & $\begin{array}{l}\text { Sample } \\
3 \text { month follow-up }\end{array}$ & $\begin{array}{l}\text { Sample } \\
12 \text { month follow-up }\end{array}$ \\
\hline & $N=428$ & $N=412$ & $N=376$ \\
\hline Constant & $68(26 \%)$ & $14(18 \%)$ & $12(21 \%)$ \\
\hline NA & 168 & 336 & 319 \\
\hline \multicolumn{4}{|l|}{ Nonhysterectomy-related pain intensity: } \\
\hline Highest pain last week (0-10) & $0.0(0.0-5.8)$ & $0.0(0.0-3.8)$ & $0.0(0.0-5.0)$ \\
\hline Highest pain last week NRS $\geq 4$ & $141(33 \%)$ & $102(25 \%)[4]$ & $121(33 \%)[4]$ \\
\hline Average pain last week (0-10) & $0.0(0.0-3.0)$ & $0.0(0.0-3.0)$ & $0.0(0.0-4.0)$ \\
\hline Average pain last week NRS $\geq 4$ & $104(24 \%)$ & $82(20 \%)[5]$ & $95(26 \%)[4]$ \\
\hline \multicolumn{4}{|l|}{ Nonhysterectomy-related neuropathic pain: } \\
\hline $\mathrm{DN} 4^{\dagger} \geq 3$ & $39(10 \%)[23]$ & NA & NA \\
\hline \multicolumn{4}{|l|}{ Nonhysterectomy-related pain location: } \\
\hline Lower abdomen & $31(7 \%)$ & $33(8 \%)$ & $33(9 \%)$ \\
\hline Upper abdomen & $14(3 \%)$ & $12(3 \%)$ & $14(4 \%)$ \\
\hline Back & $66(15 \%)$ & $60(15 \%)$ & $66(18 \%)$ \\
\hline Legs & $52(12 \%)$ & $27(7 \%)$ & $39(10 \%)$ \\
\hline Breast & $14(3 \%)$ & $11(3 \%)$ & $11(3 \%)$ \\
\hline Arms & $38(9 \%)$ & $25(6 \%)$ & $32(9 \%)$ \\
\hline Shoulders & $77(18 \%)$ & $52(13 \%)$ & $54(14 \%)$ \\
\hline Head & $36(8 \%)$ & $25(6 \%)$ & $31(8 \%)$ \\
\hline Other location & $19(4 \%)$ & $16(4 \%)$ & $16(4 \%)$ \\
\hline BPI pain interference $(0-10)$ & $2.4(0.3-4.6)$ & $0.0(0.0-2.3)$ & $0.0(0.0-2.6)$ \\
\hline BPI pain interference $\geq 4$ & $138(34 \%)[18]$ & 54 (14\%) [18] & $65(18 \%)[19]$ \\
\hline \multicolumn{4}{|l|}{ Pain medication } \\
\hline No medication & $229(55 \%)$ & $288(71 \%)$ & $256(69 \%)$ \\
\hline Paracetamol & 75 (18\%) & $55(14 \%)$ & $39(11 \%)$ \\
\hline NSAID & 45 (11\%) & $20(5 \%)$ & $21(6 \%)$ \\
\hline Paracetamol \& NSAID & $21(5 \%)$ & $10(3 \%)$ & $13(3 \%)$ \\
\hline Paracetamol and/or NSAID and other medication & $30(7 \%)$ & $16(4 \%)$ & $18(5 \%)$ \\
\hline Other medication & $19(5 \%)$ & $19(5 \%)$ & $23(6 \%)$ \\
\hline Missing data & 9 & 4 & 6 \\
\hline Other pain treatment & $34(8 \%)[10]$ & $23(6 \%)[4]$ & $31(8 \%)[9]$ \\
\hline \multicolumn{4}{|l|}{ Other physical measures } \\
\hline SF36 physical activity (0-100) & $82.2(19.4)$ & $83.5(20.0)$ & $85.9(20.2)$ \\
\hline GSR \% & NA & $88.1(15.6)$ & $93.9(13.4)$ \\
\hline Active days / week, (0-7) & $4.3(2.2)$ & $4.8(2.2)$ & $4.7(2.1)$ \\
\hline Able to perform daily activities again & NA & $337(85 \%)[17]$ & $341(94 \%)[13]$ \\
\hline Time until complete reuptake daily activities (days) & NA & $44(23)$ & $69(47)$ \\
\hline
\end{tabular}

Numbers are mean (standard deviation), median (interquartile range), or number (\%) [missing data].

$\mathrm{BPI}=$ Brief Pain Inventory, DN4=neuropathic pain questionnaire, GSR \%=global surgical recovery index, 0-100\%, $\mathrm{NA}=$ not applicable, NRS=numeric rating scale, NSAID=nonsteroidal anti-inflammatory drug.

+ Seven self-report items (DN4-interview). 


\section{Predictors of recovery after 3 and 12 months}

Pain

Of the control variables such as hospital, age, type of anesthesia, and type of incision, only hospital reached significance at 0.10 level in bivariate analysis: CzE beta -0.785 , $p=0.06$, MMC -0.656, $p=0.13$, OMC $-0.073, p=0.98$, reference Maastricht UMC+, interaction with time not shown. Besides baseline hysterectomy-related pain, also nonhysterectomy-related preoperative pain, ASA classification, number of pregnancies (gravidity) including interaction with time, expectations about hysterectomy (relief/neutral/loss), expected pain on postoperative day 4, number of close friends/relatives (MOS-SSS), surgery-related worries, general psychological robustness, having undergone prolapse surgery, acute pain at the postanesthesia care unit (PACU), NPP on postoperative day 4, and surgery-related infection during 3- and 12-month follow-up including interaction with time were entered in the multivariate model.

Model 1, based on the control variables including time and supplemented with the preoperative predictors, revealed significant differences in the risk of CPSP between the participating hospitals. Also time (12 months compared with 3 months), baseline hysterectomy-related and nonrelated highest pain, and surgery-related worries were predictors of CPSP. Finally, gravidity decreased the risk of CPSP at 12 months ( $N=314$, AIC 354.9).

Table 5. Final multivariate models for predictors of pain, physical functioning, and surgical recovery 3 and 12 months after hysterectomy

\begin{tabular}{llll}
\hline Predictors & Pain $^{\dagger}$ & Physical functioning & Surgical recovery \\
& $\mathrm{N}=253$ & $\mathrm{~N}=297$ & $\mathrm{~N}=261$ \\
& Beta (SD) & Est (SD) & Est (SD) \\
\hline Hospital & & & \\
MUMC+ & Reference & Reference & Reference \\
CzE & $-0.594(0.410)$ & $5.450(4.697)$ & $1.239(1.533)$ \\
MMC & $-0.721(0.459)$ & $6.124(5.236)$ & $-1.825(1.803)$ \\
OMC & $-1.307(0.797)$ & $18.764(7.483)^{*}$ & $0.057(2.460)$ \\
Age & $-0.014(0.025)$ & $0.139(0.136)$ & $-0.046(0.093)$ \\
Anesthesia & & & \\
GA & Reference & Reference & Reference \\
GA and epidural / Spinal / GA and spinal & $0.358(0.496)$ & $2.880(2.503)$ & $3.633(1.667)^{*}$ \\
Incision & & & \\
Vaginal / LH / LAVH & Reference & Reference & Reference \\
Median lower abdominal / Pfannenstiel & $-0.438(0.481)$ & $-5.926(5.206)$ & $-7.309(3.841)$ \\
Time & & & \\
3 months & Reference & Reference & Reference \\
12 months & $1.505(0.884)$ & $5.157(3.064)$ & $4.269(2.327)$ \\
Paid job & - & - & $-5.455(3.590)$ \\
SF36 physical functioning baseline (0-100) & - & $0.387(0.050)^{* * *}$ & - \\
\hline
\end{tabular}




\begin{tabular}{|c|c|c|c|}
\hline \multirow[t]{3}{*}{ Predictors } & Pain $^{+}$ & Physical functioning $^{\ddagger}$ & Surgical recovery ${ }^{\S}$ \\
\hline & $N=253$ & $N=297$ & $N=261$ \\
\hline & Beta (SD) & Est (SD) & Est (SD) \\
\hline \multicolumn{4}{|l|}{ ASA } \\
\hline 1 & Reference & Reference & Reference \\
\hline$\|$ & - & - & $2.677(3.163)$ \\
\hline III & - & - & $12.292(11.178)$ \\
\hline Gravidity & $0.117(0.192)$ & - & - \\
\hline \multicolumn{4}{|l|}{ Indication } \\
\hline Leiomyoma & Reference & Reference & Reference \\
\hline Prolapse & - & $0.715(5.915)$ & - \\
\hline Menorrhagia / metrorrhagia & - & $-2.101(4.543)$ & - \\
\hline Dysmenorrhoea & - & $-7.332(8.864)$ & - \\
\hline Endometriosis / adenomyosis & - & $18.103(8.413)^{*}$ & - \\
\hline Abdominal pain & - & $19.154(7.784)^{*}$ & - \\
\hline Cervical dysplasia & - & $-9.589(8.423)$ & - \\
\hline Other & - & $-16.388(7.965)^{*}$ & - \\
\hline Expected surgical recovery (0-100\%) & - & - & $0.201(0.058)^{* *}$ \\
\hline Surgery-related worries ${ }^{\prime \prime}$ & $0.500(0.159)^{* *}$ & - & $-1.904(0.660)^{* *}$ \\
\hline General psychological robustness & - & $2.332(0.964)^{*}$ & - \\
\hline History of sexual abuse & - & - & $3.754(3.927)$ \\
\hline Hysterectomy-related baseline pain (0-10) & $0.202(0.057)^{* * *}$ & - & $-0.262(0.209)$ \\
\hline Nonhysterectomy-related baseline pain (0-10) & $0.127(0.049)^{*}$ & $-0.297(0.292)$ & - \\
\hline Training gynecologist (years) & - & - & $-0.115(0.066)$ \\
\hline Neuropathic pain" postoperative day 4 & $0.921(0.365)^{*}$ & $-5.464(2.196)^{*}$ & $-3.864(1.600)^{*}$ \\
\hline Surgery-related infection ${ }^{\#} 3$ month & $1.222(0.395)^{* *}$ & - & $-13.203(4.928)^{* *}$ \\
\hline Surgery-related infection ${ }^{\#} 12$ month & - & $12.288(9.855)$ & $8.077(8.041)$ \\
\hline \multicolumn{4}{|l|}{ Interaction with Time ${ }^{+\dagger}$} \\
\hline Hospital OMC & - & $-12.835(4.365)^{* *}$ & - \\
\hline ASA III & - & - & $-14.774(6.782)^{*}$ \\
\hline Gravidity & $-0.748(0.397)^{*}$ & - & - \\
\hline Indication abdominal pain & - & $-10.755(4.537)^{*}$ & - \\
\hline Indication other & - & $9.513(4.646)^{*}$ & - \\
\hline Surgery-related infection 3 month & - & - & $6.043(2.983)^{*}$ \\
\hline Surgery-related infection 12 month & - & $-12.796(5.674)^{*}$ & $-15.928(4.863)^{* *}$ \\
\hline Intercept & $-3.228(1.420)^{*}$ & $37.726(10.043)^{* * *}$ & $69.112(7.787)^{* * *}$ \\
\hline
\end{tabular}

Numbers are Beta (SD) for pain and Estimates (SD) for physical functioning and surgical recovery.

$A S A=A m e r i c a n$ Society of Anesthesiologists, $C z E=$ Catharina Hospital Eindhoven, $G A=$ general anesthesia, $\mathrm{LAVH}=$ laparoscopic assisted vaginal hysterectomy, LH=laparoscopic hysterectomy, MUMC+=Maastricht University Medical Center+, MMC=Máxima Medical Center, OMC=Orbis Medical Center, SD=standard deviation.

${ }^{+}$Chronic postsurgical pain, hysterectomy-related highest pain last week, group no pain (NRS 0-3) versus group pain (NRS 4-10).

${ }^{\ddagger}$ Physical functioning subscale of the Short Form 36 questionnaire (SF-36, 0-100).

$\S$ GSR (0-100\%).

II Surgery-related worries (range -1.675 to 3.665) is based on regression scores, resulting from factor analysis performed on the SFQ, PCS, LOT-R, CES-D, and W-BQ12. Two factors were revealed, the first consisting of the SFQ and PCS named surgery-related worries. The second consisted of LOT-R, CES-D, and W-BQ12, named general psychological robustness (range -4.000 to 1.954 ). 
" DN4-interview, 7 self-report items, score $\geq 3$.

\# Self-reported surgery-related infection during 3- or 12-month follow-up.

${ }^{++}$Interaction with time, that is, an additional effect on 12-month compared with 3-month outcome, is only shown for significant interaction effects.

* $p<0.05$; ** $p<0.01 ; * * * 0<001$.

The results of model 2 are presented in Table 5, left column. None of the control variables reached significance anymore. The risk of CPSP was increased by baseline hysterectomy-related and nonrelated highest pain, surgery-related worries, NPP day 4, and infection at 3 months. Although the simple effects of gravidity on CPSP at 3 and 12 months were not significant, beta $0.117, p=0.54$ and $-0.632, p=0.06$, respectively, the time by gravidity interaction was $(p<0.05)$. This is in line with the near-significant effect for the 12-month outcome ( $N=253$, AIC 287.4).

\section{Physical functioning}

For the outcome physical functioning in bivariate analysis, 3 of the control variables were significant at 0.10 level. Hospital, CzE estimate 4.667, $p=0.26$, MMC 9.530, $p=0.03$, OMC 7.191, $p=0.26$, reference Maastricht UMC+. Type of anesthesia, GA with epidural 5.277, $p=0.56$, spinal $-0.407, p=0.94$, GA with spinal $-58.048, p=0.001$, reference GA. Type of incision, median lower abdominal $-9.200, p=0.25$, Pfannenstiel $-13.493, p=0.02$, vaginal $-9.582, p=0.03, \mathrm{LAVH}-0.760, p=0.88$, reference $\mathrm{LH}$. Interaction with time not shown. In addition to baseline physical functioning, marital status, employment status (paid job yes/no), ASA classification, expectations about femininity, social support (MOS-SSS total score), the psychological aggregate scores of surgery-related worries and general psychological robustness, baseline hysterectomy-related and nonrelated pain, indication, acute pain at the PACU, NPP day 4, and surgery-related postoperative infection at 12-month follow-up were entered for multivariate analyses.

Model 1, based on the control variables including time and the preoperative predictors, revealed that higher baseline levels of physical functioning and general psychological robustness, and the indication abdominal pain predicted higher postoperative levels of physical functioning. Baseline nonhysterectomy-related pain was a risk factor for lower scores. Concerning the level of physical functioning at 12 months, however, the positive effect of indication abdominal pain was attenuated by a time by indication interaction effect. Furthermore, a time by hospital interaction effect revealed that patients of 1 hospital were at increased risk for lower physical functioning scores at 12 months ( $\mathrm{N}=411$, AIC 6255.8).

Model 2 is shown in Table 5, middle column. It revealed a positive effect on physical functioning outcome for patients of 1 hospital, patients with high baseline physical function and general psychological robustness scores, and patients with indication endometriosis/adenomyosis or abdominal pain. Poorer physical functioning at follow-up was seen with indication "other," and NPP day 4. Self-reported infection at 12 months predicted lower scores at 12-month follow-up, simple effect estimate -26.101, $p=0.007$, 
simple effect at 3-month follow-up 12.288, $p=0.21$. Interaction effects of time by hospital $(p=0.004)$, time by indication abdominal pain $(p=0.02)$ or indication "other" $(p=0.04)$, and time by self-reported infection at 12 months $(p=0.03)$, indicated an attenuated effect of the corresponding reported simple effects on 12-month follow-up results ( $N=297$, AIC 4752.9).

\section{Global surgical recovery}

Only type of incision was a significant control variable at 0.10 level in bivariate analysis. Reference LH, median lower abdominal estimate -8.380, $p=0.20$, Pfannenstiel -11.427 , $p=0.02$, vaginal $-2.344, p=0.52$, LAVH 2.187, $p=0.60$, interaction with time not shown. Further added to the model were expected global surgical recovery, marital status, employment status, ASA, indication, expectations about hysterectomy, expectations about femininity, history of sexual abuse, the psychological aggregate scores surgeryrelated worries and general psychological robustness, baseline hysterectomy-related and nonrelated pain, total versus subtotal hysterectomy, experience of the attending gynecologist, NPP day 4, and surgery-related infection during 3- and 12-month followup.

Model 1 of the multilevel analyses, based on the control variables including time and the preoperative predictors, revealed that higher global surgical recovery index scores were predicted by higher expected global surgical recovery and history of sexual abuse. Risk factors were type of incision (laparotomy) and increased baseline hysterectomyrelated pain and surgery-related worries ( $N=383$, AIC 5582.8).

Model 2 is presented in Table 5, right column. Baseline global surgical recovery expectations and surgery-related worries remained significant; however, the other predictors of model 1 were exchanged for ASA classification, type of anesthesia, NPP day 4, and surgery-related infection. Patients with combined anesthesia recovered better than patients with GA only. By a significant interaction with time an attenuating effect was shown for patients of ASA class III or reporting an infection at 3 or 12 months. Patients of ASA class III recovered less well at 12 months compared with patients of ASA class I, simple effect with 12-month outcome estimate $-32.029, p=0.004$, and simple effect with 3-month outcome $12.292, p=0.27$. Patients reporting an infection at 3 or 12 months showed poorer recovery at the corresponding time. The simple effect for the 3month outcome was $-13.203, p=0.008$, and the simple effect for the 12-month outcome was -39.706, $p<0.001$ ( $N=261$, AIC 3961.1).

Multicollinearity did not play any role in the multivariate analyses of the 3 outcomes.

Conversion, complications, and major events during 3- and 12-month follow-up

Conversion of incision type during surgery occurred once in the CPSP group and 12 times in the no-CPSP group (CPSP at 3 months). The incidence of surgical complications 
such as bleeding, genitourinary tract injury, and gastrointestinal injury was relatively low and in line with recent findings. ${ }^{54}$ Blood loss $\geq 1000 \mathrm{~mL}$ occurred in 20 patients $(4.7 \%)$, genitourinary tract injury occurred in 11 patients $(2.6 \%)$, and gastrointestinal injury was reported in 1 patient $(<1 \%)$.

A surgery-related infection was reported by 50 (12.4\%) patients at 3-month followup. Assessed in a different way, 59 (14.3\%) patients reported a healthcare visit for reasons of infection or fever, not necessarily surgery-related. At 12 months, these numbers were $17(4.6 \%)$ and 20 (5.3\%), respectively. The numbers of patients who contacted their general practitioner, specialist, or the emergency department for different types of complications during follow-up are presented in Table 6 (Supplementary File, http://links.Iww.com/MD/B57). Hospitalization during 3-month follow-up was reported by 10 patients, for reasons of: pneumonia, sepsis, wound infection/abscess/hematoma (3); ileus; gastrointestinal complaints, and for flash-backs of incest induced by hysterectomy; urine retention/obstipation, ultimate diagnosis multiple sclerosis; pulmonary embolism; and stroke. At 12 months, reasons for hospitalization were gastroenteritis (2) and stroke.

\section{DISCUSSION}

The primary purpose of the present study was to examine predictors of CPSP, and secondarily of other indicators of recovery, that is, self-reported physical functioning and self-perceived global recovery. CPSP was predicted by the presence of moderate to severe hysterectomy-related and hysterectomy-unrelated pain before the operation and NPP 4 days after the operation. Other predictors were surgery-related worries and surgery-related infection. Interestingly, for the secondary outcome variables, partly different predictors were found. Preoperative pain was not significantly associated with poor physical functioning or global surgical recovery at follow-up. However, abdominal pain as the indication for hysterectomy predicted better physical functioning. Other predictors of physical functioning, besides preoperative physical functioning and indication, were participating center, general psychological robustness, NPP pain at day 4, and surgery-related infection. For global surgical recovery, we identified expected global surgical recovery, surgery-related worries, ASA classification, type of anesthesia, NPP at day 4 , and surgery-related infection as predictors.

The prominent role of preoperative pain as predictor of CPSP in the present study is congruent with many other prediction studies across various surgical procedures ${ }^{15,55}$ including hysterectomy. ${ }^{2,3,56,57}$ We used the highest pain intensity score as predictor instead of the often used mean pain intensity score. Differences were small, but highest pain proved to be the most powerful predictor. Furthermore, there is extensive evidence that high levels of pain in the acute postoperative period are associated a high prevalence of CPSP. ${ }^{15,55-57}$ In the present study, we found that especially pain with neu- 
ropathic characteristics was associated with CPSP, and additionally with physical functioning and global surgical recovery. The predictive value of surgery-related worries for CPSP is in agreement with previous studies in women undergoing hysterectomy. $3,56,57$ Moreover, a systematic review and meta-analysis revealed that preoperative fear and anxiety as well as pain catastrophizing are associated with CPSP across many different interventions. ${ }^{22}$ Because of the overlap of these measures, for the current study we created an aggregate measure representing high surgical fear and high pain catastrophizing. Similarly an aggregate score was created for the 3 more global individual difference variables, that is, optimism, depression, and well-being. This aggregate did not prove predictive of CPSP, although 2 of its constituent parts, depression and wellbeing energy, were significant $(p<0.01)$ in bivariate analyses. In contrast to the findings of several other studies, optimism was not associated with CPSP in our bivariate analyses. $^{29-31,33}$ To check whether an aggregate of only depression and well-being would be retained in the multivariate model, we performed a post hoc analysis (data not shown). This truncated aggregate did not reach significance either, suggesting that psychological factors that are more specific for the operation have greater predictive power for CPSP than global psychological states and traits. However, physical functioning was predicted by general psychological robustness. For this outcome, besides depression and wellbeing energy, the positive well-being subscale was also significant at $p=0.01$ level. Changes in physical functioning in the course of 1 year may be less directly related to the surgical intervention compared with the other outcomes in this study and additionally determined by the general psychological state of an individual.

Preoperative expectations have also been found predictive of outcome in previous studies. $^{25,42,58}$ Here, we found expected global surgical recovery to predict actual global surgical recovery, but expected pain was not predictive of CPSP. Surgery-related infection was a risk factor for physical functioning, global surgical recovery, and also CPSP. Infection may delay healing and affect all aspects of recovery. However, it should be noted that according to the original definition of CPSP, other causes for pain should be excluded. ${ }^{59}$ Our data did not allow us to assess whether reported infection was still present at the moment of pain assessment. Therefore we addressed potential confounding effects of infection with regard to outcome CPSP by including infection in the multilevel analyses.

The only other predictor showing significance across all 3 outcomes was NPP. Because NPP at day 4 contributed most to statistical model improvement, it was selected as the index for acute postsurgical pain over NRS pain and analgesia use. The obvious role of NPP suggests that at least part of the problem is persistence of NPP that already starts in the early postoperative period. ${ }^{60}$ At 3-month follow-up, the overall rate of NPP was $5 \%$ in our study, and $46 \%$ of the patients reporting CPSP indicated that their pain had neuropathic characteristics. At day 4, 53\% in the CPSP group reported NPP against $19 \%$ of patients in the no-CPSP group. Time to recovery of NPP depends on the type of nerve injury. ${ }^{61}$ Unfortunately, our data do not allow assessing whether the nerve injury 
in the no-CPSP group was less severe as compared with patients with CPSP. NPP was reported most after Pfannenstiel incision. Previously it was suggested that indeed Pfannenstiel incisions yield a higher risk of NPP compared with other types of incision. ${ }^{62-64}$

The present study provides several clues toward interventions that might reduce the incidence of CPSP after hysterectomy. We presented 2 different predictor models, with the first model including only those variables that can be obtained preoperatively. This may guide the selection of variables to be included in a future risk assessment tool. High-risk patients can be identified before surgery and offered intensified attention by the nursing staff or some form of counseling. A risk assessment tool might also guide treatment decisions regarding the surgical procedure and analgesic regime. The second model, including pre- and postoperative variables as well, gives an even more complete overview of risk factors for long-term outcome and points to the necessity of adequate postoperative pain management and infection prevention. Future studies should also establish the causality of these relations.

The results of this study also show that in general patients recovered well from hysterectomy. The prevalence of moderate to severe hysterectomy-related pain (i.e., NRS 24) was significantly lower at 3- and 12-month follow-up (10.2\% and 9.1\%) than it was before the operation (50\%). The scores of SF-36 physical functioning, between 82.4 and 85.9, are within the range as measured in female populations in the UK 86.7 (20.2), Australia 85.1 (18.7), ${ }^{65,66}$ and a sample of Dutch females 80.4 (24.2). ${ }^{36}$ Some of the other study variables also indicated generally good recovery with the number of active days/week increasing significantly from baseline to follow-up with 0.5 days. The number of days until complete reuptake of full activities was within patients' expectations, at least at 3 months, presumably in line with the prescribed 6 weeks recovery period. Overall, our results are in line with the findings of Linenberger, reporting that most patients described their physical condition 8 weeks after hysterectomy as "better than before." 8

Despite overall good outcomes, some patients still reported pain after 3 and 12 months. The prevalence of CPSP in our sample was $10.2 \%$ to $9.1 \%$, and 3 -month incidence $2.2 \%$. These numbers are well within the ranges of $5 \%$ to $32 \%$ for CPSP and $0 \%$ to $15 \%$ for acquired/increased pain at follow-up resulting from earlier reports. However, a recent investigation presented a prevalence of even $50 \%$ at 4 months after surgery. $2,3,56,57$ The high prevalence in the latter study may have resulted from the definition of CPSP, namely any pain at follow-up, whereas the other studies, including the present 1, used more stringent criteria.

This study has several strengths. First, we conducted a large-scale multicenter study with a homogeneous population, that is, females undergoing hysterectomy for benign indications. A comprehensive dataset was assessed allowing for broad analyses of predictors and the course of recovery. For future studies, the results can help to select the most appropriate predictor and outcome measures. Furthermore, based on this extensive baseline and recovery data, preoperative counseling can be further tailored to 
future patients. Second, compliance was high with $88 \%$ and $80 \%$ of patients providing follow-up data. Third, we created psychological aggregate scores which might yield more stable outcomes. Many psychological factors are correlated and depending on the specific sample and study, 1 factor might prevail in the multivariate model in one study and another in the next study. But our study still has some limitations. First, to avoid bias patients who underwent resurgery were considered as dropout. As a result, this study cannot provide insight into causes of resurgery and related complications. Second, NPP was assessed using the 7 self-report items instead of the total DN4. Although validated as DN4-interview, sensitivity and specificity are slightly lower as compared with the 10-item DN4. Third, all psychological assessments were performed by selfassessment. Given the fact that $29 \%$ of the patients indicated a history of psychological problems, a clinical diagnosis of current psychopathology would have strengthened our conclusions. Fourth, a large number of baseline data were explored for predictor analyses. As a result, type 1 errors cannot be excluded and inferences with regard to our study population should be made with caution. However, aiming at the development of suitable and comprehensive prediction models for the different outcomes, we notice that despite the risk of type 1 error, narrowing down from a broad scope of potential predictors, many of the established predictors confirmed the results of previous research.

\section{Practical implications}

Future interventional studies should aim at further reduction of perioperative pain by using interventions on modifiable psychological and physical factors. This is not only of great clinical relevance, but could provide evidence for the causal status of the proposed predictors. Studies on dedicated acute pain treatment programs would allow evaluating whether patients' recovery would indeed improve in the case of further postoperative pain reduction. Special attention should be paid to the prevention of NPP. A debate about surgical technique should be initiated among gynecologists, aiming at further reduction of iatrogenic NPP. ${ }^{67}$ Additionally, there is growing evidence that besides reduction of acute pain, perioperative treatment with pregabalin or gabapentin might prevent CPSP. This effect is mainly contributed to the efficacy of pregabalin and gabapentin in the treatment of NPP. ${ }^{68,69}$ Also other anesthesiological treatment options such as epidural analgesia or patient-controlled analgesia might need reconsideration. ${ }^{70}$ Because the length of hospital stay has now been shortened to 2.5 days on average, this might preclude certain patients in need for prolonged acute pain treatment from optimal postoperative care. Besides continued attention for postoperative pain, nurses should be taught to recognize the occurrence of NPP from the day of surgery. Finally, another hypothesis deserving further study is whether reduction of preoperative pain by prolonged, pro-active, preoperative pain treatment can reduce acute postoperative 
and, subsequently, chronic pain. The rationale is the scarcity of studies on preemptive pain treatment starting earlier than $24 \mathrm{~h}$ preoperative.

Further gains in postoperative outcomes may be derived from targeting psychological risk factors pre- or immediately postoperatively. The present study again pointed to the important role of preoperative anxiety and catastrophizing, here combined in a single surgical worries variable. Brief preoperative psychological interventions, directed at diminishing anxiety and negative cognitions relating to surgery and its outcomes, may prove effective in reducing both acute postoperative pain as well as CPSP and related long-term outcomes. ${ }^{71-73}$ In addition, prehabilitation programs based on nurse counseling or physical therapy, for example, have proven successful in improving physical or mental status in different populations. ${ }^{74-76}$ For hysterectomy patients, interventions to improve their physical condition and expectations upon recovery should be assessed and selection criteria for patients who would benefit most should be established. Postoperative care after discharge can be optimized by web-based information provision to discharged women. ${ }^{77}$ Web-based survey requests for hysterectomy patients to enter their health status on a daily basis during the 1st week postoperatively might also improve postoperative (tele) monitoring despite early discharge. Concerning postoperative complications extra attention is needed for prevention of infection and urinary tract complaints. ${ }^{67,78}$ Because of the large impact of infection on recovery, preoperative counseling should pay more attention to this aspect. Finally, consensus meetings should be initiated focusing on the definition of postoperative recovery, including which outcome measure(s) to use.

\section{Conclusion}

Predictors of CPSP are moderate to high baseline levels of pain, acute postsurgical pain on day 4, and surgery-related infection. Of the preoperative psychological factors, surgery-related worries were predictive of CPSP. Overall, recovery after hysterectomy was good in terms of postoperative pain, physical functioning, and self-perceived recovery. However, 1 out of 10 patients suffered from CPSP, frequently characterized by an NPP component. Further improvements in perioperative care should aim at optimizing baseline condition and further reducing acute postsurgical pain and surgery-related infection.

\section{ACKNOWLEDGEMENTS}

The authors like to thank A. van Ommering (MMC) for her contribution to patient inclusion and data collection, and S. van Riet (Maastricht University) for her support with data logistics. Furthermore we thank dr. M. Bergmans for facilitating the data collection of two Maastricht UMC+ patients who had their surgery at the Laurentius hospital 
Chapter 5

Roermond. Finally we thank M. Willemsen, L. Muntinga, N.A.M. Oversier, R.V.G. Soekhoe, B. Vreeswijk, M. Herman, and B.A. de Waal for their contribution to patient inclusion and data collection during their traineeship or residentship. 


\section{REFERENCES}

1. Statistics Netherlands (CBS). Operaties in het ziekenhuis; soort opname, leeftijd en geslacht, 1995-2010 2010 22-11-2014]; Available from: http://statline.cbs.nl/Statweb/publication/?DM=SLNL\&PA=80386 NED\&D1=a\&D2=|\&D3=2-3\&D4=0,16-17\&D5=|\&HDR=T\&STB=G1,G4,G2,G3\&VW=T.

2. Brandsborg B, Nikolajsen $L$, Kehlet $H$, et al. Chronic pain after hysterectomy. Acta Anaesthesiol Scand. 2008; 52(3): 327-331.

3. Pinto PR, Mclntyre T, Nogueira-Silva C, et al. Risk factors for persistent postsurgical pain in women undergoing hysterectomy due to benign causes: a prospective predictive study. J Pain. 2012; 13(11): 1045-1057.

4. Brandsborg B, Nikolajsen L, Hansen CT, et al. Risk factors for chronic pain after hysterectomy: a nationwide questionnaire and database study. Anesthesiology. 2007; 106(5): 1003-1012.

5. Fry $\mathrm{DE}$, Pine $\mathrm{M}$, Jones $\mathrm{BL}$, et al. The impact of ineffective and inefficient care on the excess costs of elective surgical procedures. J Am Coll Surg. 2011; 212(5): 779-786.

6. Lamvu G Role of Hysterectomy in the Treatment of Chronic Pelvic Pain. Obstet Gynecol. 2011; 117(5): 1175-1178.

7. Lee L, Tran T, Mayo NE, et al. What does it really mean to "recover" from an operation? Surgery. 2014; 155(2): 211-216.

8. Linenberger H, Cohen SM From hysterectomy to historicity. Health Care Women Int. 2004; 25(4): 349357.

9. Allvin R, Berg K, Idvall E, et al. Postoperative recovery: a concept analysis. J Adv Nurs. 2007; 57(5): 552558.

10. Allvin R, Ehnfors $M$, Rawal $N$, et al. Experiences of the postoperative recovery process: an interview study. Open Nurs J. 2008; 2: 1-7.

11. Allvin R, Ehnfors M, Rawal N, et al. Development of a questionnaire to measure patient-reported postoperative recovery: content validity and intra-patient reliability. J Eval Clin Pract. 2009; 15(3): 411-419.

12. Allvin R, Svensson E, Rawal N, et al. The Postoperative Recovery Profile (PRP) - a multidimensional questionnaire for evaluation of recovery profiles. J Eval Clin Pract. 2011; 17(2): 236-243.

13. Turk DC, Dworkin RH, Allen RR, et al. Core outcome domains for chronic pain clinical trials: IMMPACT recommendations. Pain. 2003; 106(3): 337-345.

14. VanDenKerkhof EG, Peters ML, Bruce J Chronic pain after surgery: time for standardization? A framework to establish core risk factor and outcome domains for epidemiological studies. Clin J Pain. 2013; 29(1): 2-8.

15. Katz J, Seltzer Z Transition from acute to chronic postsurgical pain: risk factors and protective factors. Expert Rev Neurother. 2009; 9(5): 723-744.

16. Aasvang EK, Luna IE, Kehlet $\mathrm{H}$ Challenges in postdischarge function and recovery: the case of fast-track hip and knee arthroplasty. Br J Anaesth. 2015; 115(6): 861-866.

17. McKenzie LH, Simpson J, Stewart M A systematic review of pre-operative predictors of post-operative depression and anxiety in individuals who have undergone coronary artery bypass graft surgery. Psychol Health Med. 2010; 15(1): 74-93.

18. Peters ML, Sommer M, de Rijke JM, et al. Somatic and psychologic predictors of long-term unfavorable outcome after surgical intervention. Ann Surg. 2007; 245(3): 487-494.

19. Carver CS, Pozo C, Harris SD, et al. How coping mediates the effect of optimism on distress: a study of women with early stage breast cancer. J Pers Soc Psychol. 1993; 65(2): 375-390.

20. Smith BW, Zautra AJ The role of purpose in life in recovery from knee surgery. Int J Behav Med. 2004; 11(4): 197-202.

21. Sommer M, de Rijke JM, van Kleef $M$, et al. Predictors of acute postoperative pain after elective surgery. Clin J Pain. 2010; 26(2): 87-94

22. Theunissen $\mathrm{M}$, Peters $\mathrm{ML}$, Bruce J, et al. Preoperative anxiety and catastrophizing: a systematic review and meta-analysis of the association with chronic postsurgical pain. Clin J Pain. 2012; 28(9): 819-841. 


\section{Chapter 5}

23. Hinrichs-Rocker A, Schulz K, Jarvinen I, et al. Psychosocial predictors and correlates for chronic postsurgical pain (CPSP) - a systematic review. Eur J Pain. 2009; 13(7): 719-730.

24. Shipton EA The transition from acute to chronic post surgical pain. Anaesth Intensive Care. 2011; 39(5): 824-836.

25. Sullivan $M$, Tanzer $M$, Reardon $G$, et al. The role of presurgical expectancies in predicting pain and function one year following total knee arthroplasty. Pain. 2011; 152(10): 2287-2293.

26. Waljee J, McGlinn EP, Sears ED, et al. Patient expectations and patient-reported outcomes in surgery: a systematic review. Surgery. 2014; 155(5): 799-808.

27. Davis DA, Luecken $L$, Zautra AJ Are reports of childhood abuse related to the experience of chronic pain in adulthood? A meta-analytic review of the literature. Clin J Pain. 2005; 21(5): 398-405.

28. Kaliray P, Drife J Childhood sexual abuse and subsequent gynaecological conditions. TOG. 2004; 6: 2092014.

29. Hetmann F, Kongsgaard UE, Sandvik L, et al. Prevalence and predictors of persistent post-surgical pain 12 months after thoracotomy. Acta Anaesthesiol Scand. 2015; 59(6): 740-748.

30. Hoofwijk DM, Fiddelers AA, Peters ML, et al. Prevalence and Predictive Factors of Chronic Postsurgical Pain and Poor Global Recovery 1 Year After Outpatient Surgery. Clin J Pain. 2015; 31(12): 1017-1025.

31. Powell R, Johnston M, Smith WC, et al. Psychological risk factors for chronic post-surgical pain after inguinal hernia repair surgery: a prospective cohort study. Eur J Pain. 2012; 16(4): 600-610.

32. Hanley MA, Jensen MP, Ehde DM, et al. Psychosocial predictors of long-term adjustment to lower-limb amputation and phantom limb pain. Disabil Rehabil. 2004; 26(14-15): 882-893.

33. Bruce J, Thornton AJ, Powell R, et al. Psychological, surgical, and sociodemographic predictors of pain outcomes after breast cancer surgery: a population-based cohort study. Pain. 2014; 155(2): 232-243.

34. von Elm E, Altman DG, Egger M, et al. The Strengthening the Reporting of Observational Studies in Epidemiology (STROBE) Statement: Guidelines for reporting observational studies. Int J Surg. 2014; 12: 1495-1499.

35. Berg van den J, Wulp van der C, Rapport van de Werkgroep Revisie POLS-Gezondheidsenquête 1999. 2003, Statistics Netherlands: Heerlen.

36. Aaronson NK, Muller M, Cohen PD, et al. Translation, validation, and norming of the Dutch language version of the SF-36 Health Survey in community and chronic disease populations. J Clin Epidemiol. 1998; 51(11): 1055-1068.

37. Ware JE, Jr., Sherbourne CD The MOS 36-item short-form health survey (SF-36). I. Conceptual framework and item selection. Medical care. 1992; 30(6): 473-483.

38. Cleeland CS, Ryan KM Pain assessment: global use of the Brief Pain Inventory. Ann Acad Med Singapore. 1994; 23(2): 129-138.

39. Bouhassira D, Attal N, Alchaar H, et al. Comparison of pain syndromes associated with nervous or somatic lesions and development of a new neuropathic pain diagnostic questionnaire (DN4). Pain. 2005; 114(12): 29-36.

40. Kleinbeck SV Self-reported at-home postoperative recovery. Research in nursing \& health. 2000; 23(6): 461-472.

41. Maeland JG, Havik OE Psychological predictors for return to work after a myocardial infarction. J Psychosom Res. 1987; 31(4): 471-481.

42. Mondloch MV, Cole DC, Frank JW Does how you do depend on how you think you'll do? A systematic review of the evidence for a relation between patients' recovery expectations and health outcomes. CMAJ. 2001; 165(2): 174-179.

43. Ferroni P, Deeble J Women's subjective experience of hysterectomy. Aust Health Rev. 1996; 19(2): 4055.

44. Ryan MM Hysterectomy: social and psychosexual aspects. Baillieres Clin Obstet Gynaecol. 1997; 11(1): 23-36.

45. Theunissen M, Peters ML, Schouten EG, et al. Validation of the Surgical Fear Questionnaire in Adult Patients Waiting for Elective Surgery. PLoS One. 2014; 9(6): e100225. 
46. Sullivan MJL, Bishop SR, Pivik J The Pain Catastrophizing Scale: Development and validation. Psychol Assessment. 1995; 7(4): 524-532.

47. Scheier MF, Carver CS, Bridges MW Distinguishing optimism from neuroticism (and trait anxiety, selfmastery, and self-esteem): A reevaluation of the Life Orientation Test. J Pers Soc Psychol. 1994; 67(6): 1063-1078.

48. Sherbourne CD, Stewart AL The MOS social support survey. Soc Sci Med. 1991; 32(6): 705-714.

49. Radloff LS The CES-D Scale: A self-report depression scale for research in the general population. Appl Psych Meas. 1977; 1(3): 385-401.

50. Bradley C The 12-Item Well-Being Questionnaire: origins, current stage of development, and availability. Diabetes Care. 2000; 23(6): 875.

51. Thombs BD, Bernstein DP, Ziegelstein RC, et al. A brief two-item screener for detecting a history of physical or sexual abuse in childhood. Gen Hosp Psychiatry. 2007; 29(1): 8-13.

52. Dihle A, Helseth S, Paul SM, et al. The exploration of the establishment of cutpoints to categorize the severity of acute postoperative pain. Clin J Pain. 2006; 22(7): 617-624.

53. Gerbershagen HJ, Rothaug J, Kalkman CJ, et al. Determination of moderate-to-severe postoperative pain on the numeric rating scale: a cut-off point analysis applying four different methods. Br J Anaesth. 2011; 107(4): 619-626.

54. Clarke-Pearson DL, Geller EJ Complications of hysterectomy. Obstet Gynecol. 2013; 121(3): 654-673.

55. Reddi D, Curran N Chronic pain after surgery: pathophysiology, risk factors and prevention. Postgrad Med J. 2014; 90(1062): 222-227; quiz 226.

56. Brandsborg B, Dueholm M, Nikolajsen L, et al. A prospective study of risk factors for pain persisting 4 months after hysterectomy. Clin J Pain. 2009; 25(4): 263-268.

57. VanDenKerkhof EG, Hopman WM, Goldstein DH, et al. Impact of perioperative pain intensity, pain qualities, and opioid use on chronic pain after surgery: a prospective cohort study. Reg Anesth Pain Med. 2012; 37(1): 19-27.

58. Laferton JA, Auer CJ, Shedden-Mora MC, et al. Factors associated with disability expectations in patients undergoing heart surgery. Int J Behav Med. 2015; 22(1): 85-91.

59. Macrae WA Chronic pain after surgery. Br J Anaesth. 2001; 87(1): 88-98.

60. Lavand'homme PM, Grosu I, France MN, et al. Pain trajectories identify patients at risk of persistent pain after knee arthroplasty: an observational study. Clin Orthop Relat Res. 2014; 472(5): 1409-1415.

61. Bradshaw AD, Advincula AP Postoperative neuropathy in gynecologic surgery. Obstet Gynecol Clin North Am. 2010; 37(3): 451-459.

62. Huikeshoven FJ, Dukel $L$ The bikini incision: nice, but not without painful complications. Ned Tijdschr Geneeskd. 1998; 142(26): 1481-1483.

63. Loos MJ, Scheltinga MR, Mulders LG, et al. The Pfannenstiel incision as a source of chronic pain. Obstet Gynecol. 2008; 111(4): 839-846.

64. Luijendijk RW, Jeekel J, Storm RK, et al. The low transverse Pfannenstiel incision and the prevalence of incisional hernia and nerve entrapment. Ann Surg. 1997; 225(4): 365-369.

65. Jenkinson C, Stewart-Brown S, Petersen S, et al. Assessment of the SF-36 version 2 in the United Kingdom. J Epidemiol Community Health. 1999; 53(1): 46-50.

66. Mishra G, Kuh D Sexual functioning throughout menopause: the perceptions of women in a British cohort. Menopause. 2006; 13(6): 880-890.

67. Hodges KR, Davis BR, Swaim LS Prevention and management of hysterectomy complications. Clin Obstet Gynecol. 2014; 57(1): 43-57.

68. Clarke H, Bonin RP, Orser BA, et al. The prevention of chronic postsurgical pain using gabapentin and pregabalin: a combined systematic review and meta-analysis. Anesth Analg. 2012; 115(2): 428-442.

69. Mishriky BM, Waldron NH, Habib AS Impact of pregabalin on acute and persistent postoperative pain: a systematic review and meta-analysis. Br J Anaesth. 2015; 114(1): 10-31.

70. Gilron I, Kehlet H Prevention of chronic pain after surgery: new insights for future research and patient care. Can J Anaesth. 2014; 61(2): 101-111. 


\section{Chapter 5}

71. Burns J, Moric M Psychosocial factors appear to predict postoperative pain: Interesting, but how can such information be used to reduce risk? Tech Reg Anesth Pain Manag. 2011; 15(3): 90-99.

72. Lim YC, Yobas $P$, Chen HC Efficacy of relaxation intervention on pain, self-efficacy, and stress-related variables in patients following total knee replacement surgery. Pain Manag Nurs. 2014; 15(4): 888-896.

73. Riddle DL, Keefe FJ, Nay WT, et al. Pain coping skills training for patients with elevated pain catastrophizing who are scheduled for knee arthroplasty: a quasi-experimental study. Arch Phys Med Rehabil. 2011; 92(6): 859-865.

74. Furze G, Dumville JC, Miles JN, et al. "Prehabilitation" prior to CABG surgery improves physical functioning and depression. Int J Cardiol. 2009; 132(1): 51-58.

75. Santa Mina D, Clarke H, Ritvo $P$, et al. Effect of total-body prehabilitation on postoperative outcomes: a systematic review and meta-analysis. Physiotherapy. 2014; 100(3): 196-207.

76. Tsimopoulou I, Pasquali S, Howard R, et al. Psychological Prehabilitation Before Cancer Surgery: A Systematic Review. Ann Surg Oncol. 2015; 22(13): 4117-4123.

77. Dukeshire S, Gilmour D, MacDonald N, et al. Development and evaluation of a web site to improve recovery from hysterectomy. Comput Inform Nurs. 2012; 30(3): 164-175; quiz 176-167.

78. Astagneau P, Rioux C, Golliot F, et al. Morbidity and mortality associated with surgical site infections: results from the 1997-1999 INCISO surveillance. J Hosp Infect. 2001; 48(4): 267-274. 


\section{SUPPLEMENTARY FILE}

Table 1B. Baseline characteristics per subgroup CPSP and No CPSP at 3 and 12 months

\begin{tabular}{|c|c|c|c|c|}
\hline \multirow[b]{2}{*}{ Measures } & \multicolumn{2}{|c|}{3 month follow-up } & \multicolumn{2}{|c|}{12 month follow-up } \\
\hline & $\begin{array}{l}\text { CPSP group } \\
\mathrm{n}=42\end{array}$ & $\begin{array}{l}\text { No-CPSP group } \\
n=368\end{array}$ & $\begin{array}{l}\text { CPSP group } \\
\mathrm{n}=34\end{array}$ & $\begin{array}{l}\text { No-CPSP group } \\
\mathrm{n}=341\end{array}$ \\
\hline \multicolumn{5}{|l|}{ Center } \\
\hline Maastricht UMC+ & 17 & 98 & 16 & 93 \\
\hline $\mathrm{CZE}$ & 11 & 139 & 8 & 122 \\
\hline MMC & 9 & 100 & 8 & 96 \\
\hline OMC & 5 & 31 & 2 & 30 \\
\hline Age & $45.4(6.5)$ & $47.2(7.1)$ & $45.8(8.8)$ & $47.4(7.1)$ \\
\hline Native country NL & $36(86 \%)$ & $350(95 \%)[1]$ & $28(82 \%)$ & $325(96 \%)[1]$ \\
\hline Marital status: living together & $38(91 \%)$ & $315(86 \%)[1]$ & $30(91 \%)[1]$ & $292(86 \%)[1]$ \\
\hline Employment: paid job & $22(52 \%)$ & 277 (76\%) [2] & $21(62 \%)$ & $254(75 \%)[1]$ \\
\hline \multicolumn{5}{|l|}{ Education: } \\
\hline no / lower & $10(24 \%)$ & $53(14 \%)$ & $4(12 \%)$ & 49 (15\%) \\
\hline intermediate & $22(52 \%)$ & $215(59 \%)$ & $18(53 \%)$ & $202(59 \%)$ \\
\hline university / higher & $10(24 \%)$ & $99(27 \%)$ & $12(35 \%)$ & $89(26 \%)$ \\
\hline missing data & 0 & 1 & 0 & 1 \\
\hline \multicolumn{5}{|l|}{ General health: } \\
\hline very poor / poor & $2(5 \%)$ & $2(1 \%)$ & $1(3 \%)$ & $4(1 \%)$ \\
\hline moderate & $15(36 \%)$ & $57(15 \%)$ & $8(24 \%)$ & $56(16 \%)$ \\
\hline very good / good & $25(59 \%)$ & $308(84 \%)$ & $25(73 \%)$ & $281(83 \%)$ \\
\hline missing data & 0 & 1 & 0 & 0 \\
\hline \multicolumn{5}{|l|}{ Smoking: } \\
\hline yes & $13(31 \%)$ & $75(21 \%)$ & $9(27 \%)$ & $71(21 \%)$ \\
\hline no & $14(33 \%)$ & $150(41 \%)$ & $10(29 \%)$ & $140(42 \%)$ \\
\hline stopped & $15(36 \%)$ & $138(38 \%)$ & $15(44 \%)$ & $126(37 \%)$ \\
\hline missing data & 0 & 5 & 0 & 4 \\
\hline \multicolumn{5}{|l|}{ ASA I / II / III } \\
\hline । & $23(56 \%)$ & $200(57 \%)$ & $22(65 \%)$ & $190(58 \%)$ \\
\hline$\|$ & $16(39 \%)$ & $148(42 \%)$ & $10(29 \%)$ & $132(41 \%)$ \\
\hline III & $2(5 \%)$ & $4(1 \%)$ & $2(6 \%)$ & $4(1 \%)$ \\
\hline missing data & 1 & 16 & 0 & 15 \\
\hline Body Mass Index & $26.8(4.7)$ & $26.4(4.9)$ & $25.7(4.0)$ & $26.6(5.0)$ \\
\hline \multicolumn{5}{|l|}{ Medical history: } \\
\hline chronic disease & $18(43 \%)$ & $131(36 \%)$ & $15(44 \%)$ & $120(35 \%)$ \\
\hline psychological problems & $14(33 \%)$ & $103(28 \%)[3]$ & $11(32 \%)$ & $91(27 \%)[2]$ \\
\hline surgery last year & $15(36 \%)$ & $96(26 \%)[5]$ & $10(29 \%)$ & $88(26 \%)[2]$ \\
\hline hysterectomy-related surgery last year & $10(24 \%)$ & $64(18 \%)[4]$ & $9(27 \%)$ & $58(17 \%)[1]$ \\
\hline \multicolumn{5}{|c|}{ Gynecological history: } \\
\hline pre-menopausal state & $33(79 \%)$ & $273(76 \%)[8]$ & $22(69 \%)[2]$ & $256(76 \%)[4]$ \\
\hline hormonal replacement therapy & $0(0 \%)$ & $15(4 \%)[3]$ & $1(3 \%)$ & $13(4 \%)[1]$ \\
\hline contraception use & $7(17 \%)$ & $77(21 \%)[3]$ & $9(27 \%)$ & 65 (19\%) [2] \\
\hline gravidity & $36(88 \%)[1]$ & $301(83 \%)[6]$ & $27(82 \%)[1]$ & $283(84 \%)[5]$ \\
\hline parity & $1.9(1.3)$ & $1.8(1.1)$ & $1.6(1.0)$ & $1.8(1.1)$ \\
\hline
\end{tabular}




\begin{tabular}{|c|c|c|c|c|}
\hline \multirow[b]{2}{*}{ Measures } & \multicolumn{2}{|c|}{3 month follow-up } & \multicolumn{2}{|c|}{12 month follow-up } \\
\hline & $\begin{array}{l}\text { CPSP group } \\
n=42\end{array}$ & $\begin{array}{l}\text { No-CPSP group } \\
n=368\end{array}$ & $\begin{array}{l}\text { CPSP group } \\
n=34\end{array}$ & $\begin{array}{l}\text { No-CPSP group } \\
n=341\end{array}$ \\
\hline vaginal delivery or caesarean section & $35(83 \%)$ & $300(83 \%)[5]$ & $26(79 \%)[1]$ & $283(84 \%)[3]$ \\
\hline caesarean section & $8(19 \%)$ & $51(14 \%)[2]$ & $4(12 \%)$ & $46(14 \%)[1]$ \\
\hline sexually active & $33(79 \%)$ & 297 (84\%) [16] & $27(79 \%)$ & $275(84 \%)[15]$ \\
\hline \multicolumn{5}{|l|}{ Indication (more than 1 possible): } \\
\hline leiomyoma & 12 & 120 & 15 & 106 \\
\hline prolapse & 8 & 65 & 3 & 65 \\
\hline menorrhagia / metrorrhagia & 22 & 172 & 18 & 163 \\
\hline dysmenorrhoea & 4 & 22 & 2 & 18 \\
\hline endometriosis / adenomyosis & 1 & 21 & 1 & 18 \\
\hline abdominal pain & 3 & 23 & 6 & 17 \\
\hline cervical dysplasia & 3 & 18 & 2 & 19 \\
\hline other & 4 & 21 & 3 & 19 \\
\hline
\end{tabular}

CPSP: chronic postsurgical pain, at 3 and 12 months, hysterectomy-related highest pain last week, cut-off NRS $\geq 4$. At 3 month $n=412$ but because of 2 cases with missing data on the variable defining CPSP, $n$ of the subgroups with and without CPSP count up to 410 . At 12 months $n=376$ but because of 1 case with missing data on the variable defining CPSP, $\mathrm{n}$ of the subgroups with and without CPSP count up to 375 .

Maastricht UMC+: Maastricht University Medical Center+; CzE: Catharina hospital Eindhoven; MMC: Máxima Medical Center, Veldhoven; OMC: Orbis Medical Center, Sittard-Geleen.

Numbers are mean (SD) or number (\%), [missing data].

Medical history, psychological problems: answer yes to "Did you ever suffer from psychological problems for which you needed support?" 
Table 2B. Psychosocial baseline measures per subgroup CPSP and No CPSP at 3 and 12 months.

\begin{tabular}{|c|c|c|c|c|}
\hline \multirow{3}{*}{ Baseline measures } & \multicolumn{2}{|c|}{ Sample 3 month follow-up } & \multicolumn{2}{|c|}{ Sample 12 month follow-up } \\
\hline & CPSP group & No-CPSP group & CPSP group & No-CPSP group \\
\hline & $N=42$ & $N=368$ & $N=34$ & $N=342$ \\
\hline Expected pain after 4 days $(0-10)$ & $6(4-7)$ & $5(3-6)$ & $6(3-7)$ & $5(3-6)$ \\
\hline Expected pain after 4 days NRS $\geq 4$ & $33(85 \%)[3]$ & $260(72 \%)[5]$ & $25(74 \%)$ & $243(73 \%)[8]$ \\
\hline Expected GSR \% after 3months & $89.9(11.5)$ & $93.8(10.3)$ & $90.9(15.5)$ & $93.1(11.0)$ \\
\hline Expected number of days until full activities & $47(40)$ & $44(30)$ & $47(26)$ & $43(32)$ \\
\hline \multicolumn{5}{|l|}{ Expectation hysterectomy: } \\
\hline relief & $36(88 \%)$ & $268(74 \%)$ & $28(82 \%)$ & $248(74 \%)$ \\
\hline neutral & $3(7 \%)$ & $87(24 \%)$ & $3(9 \%)$ & $81(24 \%)$ \\
\hline loss & $2(5 \%)$ & $7(2 \%)$ & $3(9 \%)$ & $7(2 \%)$ \\
\hline missing data & 1 & 6 & 0 & 5 \\
\hline \multicolumn{5}{|l|}{ Expectation less femininity: } \\
\hline not at all / a bit less & $38(95 \%)$ & $358(98 \%)$ & $33(97 \%)$ & $331(98 \%)$ \\
\hline rather much less / very much less & $2(5 \%)$ & $7(2 \%)$ & $1(3 \%)$ & $7(2 \%)$ \\
\hline missing data & 2 & 3 & 0 & 3 \\
\hline Surgical fear short term (0-40) & $17.8(10.8)$ & $15.6(9.7)$ & $21.3(10.6)$ & $15.5(9.8)$ \\
\hline Surgical fear long term $(0-40)$ & $12.1(8.7)$ & $9.0(8.4)$ & $12.8(10.6)$ & $9.2(8.3)$ \\
\hline PCS (0-52) & $15.2(8.6)$ & $11.7(8.1)$ & $17.7(9.0)$ & $11.5(8.1)$ \\
\hline LOT-R (0-24) & $17.7(3.8)$ & $17.0(3.7)$ & $16.8(3.9)$ & $17.0(3.6)$ \\
\hline MOS-SSS number of persons & $12.0(10.9)$ & $9.7(6.2)$ & $9.4(6.9)$ & $10.2(7.2)$ \\
\hline MOS-SSS total $(0-100)$ & $89.4(14.0)$ & $85.5(18.5)$ & $87.7(15.8)$ & $85.6(18.6)$ \\
\hline CES-D (0-60) & $14.1(9.0)$ & $10.3(7.9)$ & $16.5(9.8)$ & $10.2(7.8)$ \\
\hline WBQ energy (0-12) & $6.1(2.7)$ & $7.4(2.7)$ & $6.2(3.0)$ & $7.4(2.7)$ \\
\hline WBQ positive well-being (0-12) & $7.0(2.7)$ & $7.8(2.9)$ & $6.9(2.7)$ & $7.7(2.9)$ \\
\hline History of physical abuse & $4(10 \%)$ & $44(12 \%)[3]$ & $5(15 \%)$ & $35(10 \%)[3]$ \\
\hline History of sexual abuse & $11(27 \%)[1]$ & $64(18 \%)[6]$ & $8(25 \%)[2]$ & $66(20 \%)[3]$ \\
\hline
\end{tabular}

CPSP: chronic postsurgical pain, at 3 and 12 months, hysterectomy-related highest pain last week, cut-off NRS $\geq 4$. At 3 month $n=412$ but because of 2 cases with missing data on the variable defining CPSP, $n$ of the subgroups with and without CPSP count up to 410 . At 12 months $n=376$ but because of 1 case with missing data on the variable defining CPSP, $n$ of the subgroups with and without CPSP count up to 375 .

Expected pain data are given as median (IQR), all others as mean (SD) or number (\%), [missing data].

GSR \%: Global surgical recovery index, 0-100\%; PCS: pain catastrophizing scale; LOT-R: life orientation testrevised; MOS-SSS: Medical Outcomes Study-social support survey with number of close friends/relatives and total score of the subscales tangible support, emotional/informative support, affective support, and positive social interaction; CES-D: Center for Epidemiological Studies - Depression; WBQ: well-being questionnaire, energy and positive well-being subscales. 
Chapter 5

Table 3B. Surgery and acute pain per subgroup CPSP and No CPSP at 3 and 12 months

\begin{tabular}{|c|c|c|c|c|}
\hline \multirow[b]{2}{*}{ Measures } & \multicolumn{2}{|c|}{ Sample 3 month follow-up } & \multicolumn{2}{|c|}{ Sample 12 month follow-up } \\
\hline & $\begin{array}{l}\text { CPSP group } \\
n=42\end{array}$ & $\begin{array}{l}\text { No-CPSP group } \\
n=368\end{array}$ & $\begin{array}{l}\text { CPSP group } \\
n=34\end{array}$ & $\begin{array}{l}\text { No-CPSP group } \\
\mathrm{n}=341\end{array}$ \\
\hline \multicolumn{5}{|l|}{ Anesthesia } \\
\hline GA & $36(86 \%)$ & $306(83 \%)$ & $29(85 \%)$ & $283(83 \%)$ \\
\hline GA \& epidural & $0(0 \%)$ & $14(4 \%)$ & $2(6 \%)$ & $11(3 \%)$ \\
\hline spinal & $5(12 \%)$ & $45(12 \%)$ & $3(9 \%)$ & $44(13 \%)$ \\
\hline GA \& spinal & $1(2 \%)$ & $3(1 \%)$ & $0(0 \%)$ & $3(1 \%)$ \\
\hline duration (hours) & $2: 14(0: 44)$ & $2: 18(0: 55)$ & $2: 16(0: 45)$ & $2: 15(0: 52)$ \\
\hline \multicolumn{5}{|l|}{ Hysterectomy } \\
\hline total & $39(93 \%)$ & $348(95 \%)[2]$ & $31(91 \%)$ & $323(95 \%)[2]$ \\
\hline oophorectomy & $2(5 \%)$ & $31(8 \%)$ & $1(3 \%)$ & $28(8 \%)$ \\
\hline prolapse surgery & $8(20 \%)[1]$ & $69(20 \%)[21]$ & $2(6 \%)[1]$ & $70(22 \%)[17]$ \\
\hline \multicolumn{5}{|l|}{ Incision } \\
\hline median lower abdominal & $3(7 \%)$ & $19(5 \%)$ & $1(3 \%)$ & $18(5 \%)$ \\
\hline Pfannenstiel & $4(10 \%)$ & $45(12 \%)$ & $6(18 \%)$ & $40(12 \%)$ \\
\hline vaginal & $19(45 \%)$ & $148(40 \%)$ & $14(41 \%)$ & $144(42 \%)$ \\
\hline $\mathrm{LH}$ & $7(17 \%)$ & $76(21 \%)$ & $7(20 \%)$ & $66(19 \%)$ \\
\hline LAVH & $9(21 \%)$ & $80(22 \%)$ & $6(18 \%)$ & $73(22 \%)$ \\
\hline Blood loss (ml) & $202(180)$ & $284(381)$ & $262(542)$ & $284(358)$ \\
\hline Training gynecologist (years) & $14.3(10.3)$ & $14.1(9.6)$ & $16.6(11.3)$ & $13.9(9.6)$ \\
\hline \multicolumn{5}{|l|}{ Pain PACU } \\
\hline at rest $(0-10)$ & $4.0(1.0-7.0)$ & $3.0(0.0-5.0)$ & $3.0(0.0-6.0)$ & $3.0(0.0-5.0)$ \\
\hline at rest $N R S \geq 4$ & $22(56 \%)[3]$ & 131 (40\%) [43] & $14(47 \%)[4]$ & $128(42 \%)[36]$ \\
\hline \multicolumn{5}{|l|}{ Pain postoperative day 4} \\
\hline highest pain last 24 hrs (0-10) & $5(3-6.8)$ & $3(1-4)$ & $3(1.3-6.0)$ & $3(1-5)$ \\
\hline highest pain last 24 hrs NRS $\geq 4$ & $26(72 \%)[6]$ & $114(35 \%)[45]$ & $13(46 \%)[6]$ & $110(37 \%)[43]$ \\
\hline average pain last 24 hrs $(0-10)$ & $4(2-5)$ & $2(1-3)$ & $3(1-5)$ & $2(1-3)$ \\
\hline average pain last $24 \mathrm{hrs} N R S \geq 4$ & $24(63 \%)[4]$ & $69(21 \%)[45]$ & $12(41 \%)[5]$ & $72(24 \%)[42]$ \\
\hline DN4 $\geq 3$ & $19(53 \%)[6]$ & $58(19 \%)[64]$ & $11(38 \%)[5]$ & $61(22 \%)[59]$ \\
\hline Using pain medication postoperative day 4 & $28(76 \%)[5]$ & $226(70 \%)[47]$ & $25(81 \%)[3]$ & $211(71 \%)[45]$ \\
\hline
\end{tabular}

CPSP: chronic postsurgical pain, at 3 and 12 months, hysterectomy-related highest pain last week, cut-off NRS $\geq 4$. At 3 month $n=412$ but because of 2 cases with missing data on the variable defining CPSP, $n$ of the subgroups with and without CPSP count up to 410 . At 12 months $n=376$ but because of 1 case with missing data on the variable defining CPSP, $\mathrm{n}$ of the subgroups with and without CPSP count up to 375 .

Numbers are mean (SD), median (IQR), or number (\%), [missing data].

GA: general anesthesia. Hysterectomy total: compared with subtotal hysterectomy. LH: laparoscopic hysterectomy, LAVH: laparoscopic assisted vaginal hysterectomy.

Training gynecologist: years of experience including residentship of the 1st responsible gynecologist.

PACU: Post Anesthesia Care Unit. Pain 1 hour after arrival at PACU.

DN4: neuropathic pain questionnaire, 7 self-report items (DN4-interview). 
Table 4B. Preoperative pain and physical characteristics per subgroup CPSP and No CPSP at 3 and 12 months

\begin{tabular}{|c|c|c|c|c|}
\hline \multirow[b]{2}{*}{ Baseline measures } & \multicolumn{2}{|c|}{ Sample 3 month follow-up } & \multicolumn{2}{|c|}{ Sample 12 month follow-up } \\
\hline & $\begin{array}{l}\text { CPSP group } \\
n=42\end{array}$ & $\begin{array}{l}\text { No-CPSP group } \\
n=368\end{array}$ & $\begin{array}{l}\text { CPSP group } \\
n=34\end{array}$ & $\begin{array}{l}\text { No-CPSP group } \\
n=341\end{array}$ \\
\hline \multicolumn{5}{|l|}{ Hysterectomy-related pain intensity: } \\
\hline highest pain last week $(0-10)$ & $6.5(4.8-8.0)$ & $3.0(0.0-6.0)$ & $7.0(3.0-8.0)$ & $3.0(0.0-6.0)$ \\
\hline highest pain last week NRS $\geq 4$ & $33(79 \%)$ & $169(46 \%)$ & $25(74 \%)$ & $158(46 \%)$ \\
\hline average pain last week (0-10) & $5.0(2.8-6.3)$ & $2.0(0.0-5.0)$ & $5.0(2.0-6.0)$ & $2.0(0.0-5.0)$ \\
\hline average pain last week NRS $\geq 4$ & $31(74 \%)$ & $136(37 \%)$ & $24(71 \%)$ & $125(37 \%)$ \\
\hline \multicolumn{5}{|c|}{ Hysterectomy-related neuropathic pain: } \\
\hline DN4 $\geq 3$ & $6(16 \%)[4]$ & $32(9 \%)[23]$ & $6(19 \%)[3]$ & $30(9 \%)[23]$ \\
\hline \multicolumn{5}{|l|}{ Hysterectomy-related pain location: } \\
\hline lower abdomen & $34(81 \%)$ & $200(54 \%)$ & $26(77 \%)$ & $187(55 \%)$ \\
\hline upper abdomen & $5(12 \%)$ & $15(4 \%)$ & $5(15 \%)$ & $15(4 \%)$ \\
\hline back & $26(62 \%)$ & $109(27 \%)$ & $19(56 \%)$ & $101(30 \%)$ \\
\hline legs & $10(24 \%)$ & $38(10 \%)$ & $9(27 \%)$ & $35(10 \%)$ \\
\hline breast & $4(10 \%)$ & $11(3 \%)$ & $7(21 \%)$ & $9(3 \%)$ \\
\hline other location & $5(12 \%)$ & $17(5 \%)$ & $5(15 \%)$ & $17(5 \%)$ \\
\hline \multicolumn{5}{|c|}{ Onset hysterectomy-related pain since: } \\
\hline 1 week - 3 months & $4(12 \%)$ & $32(15 \%)$ & $1(4 \%)$ & $31(16 \%)$ \\
\hline $3-12$ months & $6(17 \%)$ & $60(28 \%)$ & $4(15 \%)$ & $56(28 \%)$ \\
\hline$>12$ months & $25(71 \%)$ & $124(57 \%)$ & $22(81 \%)$ & $112(56 \%)$ \\
\hline NA & 7 & 152 & 7 & 142 \\
\hline \multicolumn{5}{|c|}{ Intermittence hysterectomy-related pain: } \\
\hline brief/short & $4(12 \%)$ & $15(7 \%)$ & $0(0 \%)$ & $16(8 \%)$ \\
\hline periodic/recurrent & $20(57 \%)$ & $147(69 \%)$ & $15(58 \%)$ & $137(70 \%)$ \\
\hline constant & $11(31 \%)$ & $51(24 \%)$ & $11(42 \%)$ & $44(22 \%)$ \\
\hline NA & 7 & 155 & 8 & 144 \\
\hline \multicolumn{5}{|c|}{ Non-hysterectomy-related pain intensity: } \\
\hline highest pain last week (0-10) & $5.5(0.0-7.0)$ & $0.0(0.0-5.0)$ & $3.5(0.0-6.3)$ & $0.0(0.0-5.0)$ \\
\hline highest pain last week NRS $\geq 4$ & $24(57 \%)$ & $111(30 \%)$ & $17(50 \%)$ & $110(32 \%)$ \\
\hline average pain last week (0-10) & $3.5(0.0-5.3)$ & $0.0(0.0-3.0)$ & $2.0(0.0-6.0)$ & $0.0(0.0-3.0)$ \\
\hline average pain last week NRS $\geq 4$ & $21(50 \%)$ & $79(22 \%)$ & $14(41 \%)$ & $78(23 \%)$ \\
\hline \multicolumn{5}{|c|}{ Non-hysterectomy-related neuropathic pain: } \\
\hline DN4 $\geq 3$ & $7(18 \%)[4]$ & $28(8 \%)[18]$ & $7(24 \%)[5]$ & $22(7 \%)[17]$ \\
\hline \multicolumn{5}{|c|}{ Non-hysterectomy-related pain location: } \\
\hline lower abdomen & $8(19 \%)$ & $21(6 \%)$ & $7(21 \%)$ & $19(6 \%)$ \\
\hline upper abdomen & $1(2 \%)$ & $11(3 \%)$ & $2(6 \%)$ & $9(3 \%)$ \\
\hline back & $14(33 \%)$ & $50(14 \%)$ & $7(21 \%)$ & $53(16 \%)$ \\
\hline legs & $9(21 \%)$ & $39(11 \%)$ & $10(29 \%)$ & $37(11 \%)$ \\
\hline breast & $5(12 \%)$ & $9(2 \%)$ & $4(12 \%)$ & $8(2 \%)$ \\
\hline arms & $7(17 \%)$ & $28(8 \%)$ & $6(18 \%)$ & $28(8 \%)$ \\
\hline shoulders & $10(24 \%)$ & $64(17 \%)$ & $10(29 \%)$ & $57(17 \%)$ \\
\hline head & $7(17 \%)$ & $27(7 \%)$ & $5(15 \%)$ & $26(8 \%)$ \\
\hline other location & $4(10 \%)$ & $15(4 \%)$ & $1(3 \%)$ & $17(5 \%)$ \\
\hline BPI pain interference $(0-10)$ & $4.4(2.8-5.7)$ & $2.0(0.1-4.3)$ & $4.3(2.1-6.2)$ & $2.0(0.1-4.4)$ \\
\hline $\mathrm{BPI}$ pain interference NRS $\geq 4$ & $23(56 \%)[1]$ & 105 (30\%) [17] & $19(56 \%)$ & $102(31 \%)[16]$ \\
\hline
\end{tabular}




\section{Chapter 5}

\begin{tabular}{|c|c|c|c|c|}
\hline \multirow[b]{2}{*}{ Baseline measures } & \multicolumn{2}{|c|}{ Sample 3 month follow-up } & \multicolumn{2}{|c|}{ Sample 12 month follow-up } \\
\hline & $\begin{array}{l}\text { CPSP group } \\
n=42\end{array}$ & $\begin{array}{l}\text { No-CPSP group } \\
n=368\end{array}$ & $\begin{array}{l}\text { CPSP group } \\
n=34\end{array}$ & $\begin{array}{l}\text { No-CPSP group } \\
n=341\end{array}$ \\
\hline Using pain medication & $25(63 \%)[2]$ & $149(42 \%)[13]$ & $21(62 \%)$ & $139(43 \%)[14]$ \\
\hline Other pain treatment & $1(3 \%)[2]$ & $32(9 \%)[8]$ & $3(9 \%)[1]$ & $24(7 \%)[9]$ \\
\hline \multicolumn{5}{|c|}{ Other physical baseline measures } \\
\hline SF36 physical activity (0-100) & $72.4(23.9)$ & $83.7(18.2)$ & $74.9(24.5)$ & $83.0(18.9)$ \\
\hline Active days / week, (0-7) & $4.5(2.4)$ & $4.3(2.2)$ & $3.9(2.2)$ & $4.3(2.2)$ \\
\hline
\end{tabular}

CPSP: chronic postsurgical pain, at 3 and 12 months, hysterectomy-related highest pain last week, cut-off NRS $\geq 4$. At 3 month $n=412$ but because of 2 cases with missing data on the variable defining CPSP, $n$ of the subgroups with and without CPSP count up to 410 . At 12 months $n=376$ but because of 1 case with missing data on the variable defining CPSP, $n$ of the subgroups with and without CPSP count up to 375 .

Numbers are median (IQR), number (\%), [missing data]. NA: not applicable.

DN4: neuropathic pain questionnaire, 7 self-report items (DN4-interview). 
Table 5B. Final multivariate models: additional legend concerning data reduction and analysis

To maintain a certain uniformity across multivariate analyses of the three outcome measures and to reduce the number of variables, for predictors with a high level of similarity or overlap an a priori choice was made regarding which one to retain. For preoperative health status we chose to use ASA classification instead of the general health status, body mass index, or the presence of chronic disease as obtained from the comorbidity screening list, because the ASA classification is readily available and clinically useful. For preoperative pain status both highest hysterectomy-related and highest non-hysterectomy-related pain (NRS) were used rather than preoperative neuropathic pain, pain interference, or pain treatment. The reason was that these variables do not overlap and best match the primary outcome variable which was also defined as the highest (hysterectomy-related) pain at 3 months. Acute postsurgical pain on day four was entered using neuropathic pain and not by NRS score or pain treatment because NPP contributed most to statistical model improvement. If bivariately significant, pain immediately after surgery, at the Post Anesthesia Care Unit (PACU), was also entered in the model. Of the postoperative complications only surgery-related infection was assessed for predictor analysis because this was assessed explicitly, in contrast to other complications, which were assessed more generally.

The following variables were significant at $p=0.10$ level, but not entered in the multivariate model because of the a priori selection of predictor variables described above: baseline general health status, physical functioning, expected global surgical recovery, SFQ long-term, PCS, CES-D, WBQ-energy, hysterectomy-related baseline pain location, non-hysterectomy-related baseline NPP and pain location, baseline pain interference, baseline analgesia use, and acute pain NRS day 0-4 with regard to the outcome CPSP. General health status, number of active days/week, expected global surgical recovery, expected number of days until reuptake of full activities, SFQ short- and long-term, CESD, both W-BQ12 subscales, hysterectomy-related baseline pain location, nonhysterectomy-related baseline NPP and pain location, pain interference, analgesia use postoperative day $0,2,3$, and acute pain NRS day 2-4 were the variables significant at $p=0.10$ but not entered for outcome physical functioning. And finally, the expected number of days until reuptake of full activities, SFQ short-term, PCS, LOT-R, CES-D, WBQ12 positive well-being subscale, expected pain, hysterectomy-related and nonrelated baseline pain location, pain interference, analgesia use at postoperative day 1-4 and acute pain NRS day 1-4 were not entered for the outcome global surgical recovery.

To further reduce the load of bivariate significant predictors on the multivariate model an intermediate step was made. Based on domain or point in time, predictors were clustered in six blocks. Block 1 consisted of the control variables hospital, age, type of incision, type of anesthesia, baseline measure or expectation of the outcome, and a time variable defining 3 and 12 month follow-up. Block 2 consisted of predictors concerning baseline health status and sociodemographic variables, block 3 consisted of gynecological history and indication for surgery, block 4 concerned psychosocial predic- 
tors, block 5 surgery and acute pain, and block 6 represented self-reported infection during follow-up. Bivariate significant predictors of each of the blocks 2-6 were added separately to the control variables of block 1 , and backward analyses were applied to select which variables of each block were selected (criterion $p<0.10$ ) for the final multivariate analyses. Two multivariate models are presented. Model 1, consisting of the predictors of block 1-4, reflects the impact of control variables and preoperative predictors. In model 2 the per- and postoperative variables of block 5 and 6 were added.

For outcome pain, bivariate analyses were performed using a random intercept model with a variance components covariance structure. If the variance of the random intercept was close to zero, analyses were repeated without random intercept. Eventually this applied to all potential predictors except sexual activity, number of close friends/relatives, and acute pain at the PACU. As a result, the multivariate analyses were also performed without random intercept.

Table 6. Health care visits for self-reported complications during 3 and 12 month follow-up

\begin{tabular}{|c|c|c|c|c|}
\hline \multirow[b]{2}{*}{ Visits } & \multicolumn{2}{|c|}{ Sample 3 month follow-up } & \multicolumn{2}{|c|}{ Sample 12 month follow-up } \\
\hline & $\begin{array}{l}\text { CPSP group } \\
n=42\end{array}$ & $\begin{array}{l}\text { No-CPSP group } \\
n=368\end{array}$ & $\begin{array}{l}\text { CPSP group } \\
\mathrm{n}=34\end{array}$ & $\begin{array}{l}\text { No-CPSP group } \\
n=341\end{array}$ \\
\hline Visit general practitioner & $15(36 \%)$ & $70(19 \%)$ & $12(35 \%)$ & $47(14 \%)$ \\
\hline Visit specialist & $20(48 \%)$ & $66(18 \%)$ & $13(38 \%)$ & $39(11 \%)$ \\
\hline Visit emergency & $4(10 \%)$ & $17(5 \%)$ & $3(9 \%)$ & $9(3 \%)$ \\
\hline \multicolumn{5}{|l|}{ Reason visit: } \\
\hline infection / fever & $16(38 \%)$ & $43(12 \%)$ & $5(15 \%)$ & $15(4 \%)$ \\
\hline urinary tract & $8(19 \%)$ & $39(11 \%)$ & $1(3 \%)$ & $15(4 \%)$ \\
\hline abdominal / gastrointestinal complaints & $9(21 \%)$ & $31(8 \%)$ & $8(24 \%)$ & $16(5 \%)$ \\
\hline pain / cramp & $11(26 \%)$ & $22(6 \%)$ & $6(18 \%)$ & $20(6 \%)$ \\
\hline cystitis & $5(12 \%)$ & $25(7 \%)$ & $0(0 \%)$ & $7(2 \%)$ \\
\hline bleeding / haematoma & $9(21 \%)$ & $19(5 \%)$ & $3(9 \%)$ & $7(2 \%)$ \\
\hline musculoskeletal complaints & $7(17 \%)$ & $13(4 \%)$ & $6(18 \%)$ & $17(5 \%)$ \\
\hline wound inspection & $2(5 \%)$ & $15(4 \%)$ & $5(15 \%)$ & $1(<1 \%)$ \\
\hline ileus / obstipation & $2(5 \%)$ & $12(3 \%)$ & $1(3 \%)$ & $2(<1 \%)$ \\
\hline Hospitalized (no surgery) & $2(5 \%)$ & $8(2 \%)$ & $0(0 \%)$ & $3(1 \%)$ \\
\hline
\end{tabular}

CPSP: chronic postsurgical pain, at 3 and 12 months, hysterectomy-related highest pain last week, cut-off NRS $\geq 4$. At 3 month $n=412$ but because of 2 cases with missing data on CPSP outcome, $n$ of the subgroups with and without CPSP count up to 410. At 12 months $n=376$ but because of 1 case with missing data on CPSP outcome, $n$ of the subgroups with and without CPSP count up to 375.

Number (\%). 


\section{Chapter}

\section{Prevalence and predictors of depression and well-being after hysterectomy: an observational study}

This chapter is submitted for publication as: Maurice Theunissen, Madelon L. Peters, Jan Schepers, Dick C. Schoot, Hans-Fritz Gramke, Marco A. Marcus. Prevalence and predictors of depression and well-being after hysterectomy: an observational study. 


\section{ABSTRACT}

Objectives: To assess risk and protective factors for depression and well-being, 3 and 12 months after elective hysterectomy. Secondary objectives were to assess the incidence of depression, level of well-being, and feelings of femininity.

Study design: A prospective multicenter cohort study was performed among 419 women, planned for hysterectomy for benign indication. Data were collected in the week prior to surgery, and in the per- and postoperative period up to the fourth postoperative day and 3 and 12 months after surgery. Sociodemographic variables, baseline health status, psychosocial predictors, and surgery data were assessed. Outcome measures were Center for Epidemiological Studies-Depression scale (CES-D, range 0$60)$, the 12-item well-being questionnaire energy and positive well-being subscales (range 0-12), and feelings of femininity. Linear mixed model analyses were performed.

Results: Levels of depression, energy, and positive well-being after hysterectomy were predicted by their corresponding baseline levels (estimate $0.62 p<0.001,0.39 p<0.001$, $0.37 p<0.001$, respectively) and baseline pain (0.31 $p=0.003,-0.09 p=0.026,-0.10$ $p=0.008$ ). Postoperative infection reported at 12 months affected CES-D and energy level. Several other gynaecological, psychosocial, or perioperative factors were also predictive for one of the outcomes. Prevalence of depression at baseline, 3 and 12 months was $24 \%, 19 \%$, and $21 \%$, respectively. In general, well-being scores were slightly higher 3 and 12 months after hysterectomy than at baseline. Feelings of femininity were not negatively affected in $92 \%$ of the patients.

Conclusions: Optimizing preoperative psychosocial status, intensified perioperative pain treatment, and reduction of postoperative infection may further improve psychological outcome after hysterectomy. In the majority of patients we observed small but significant improvements with regard to postoperative depression and well-being, while feelings of femininity were unaffected. 


\section{INTRODUCTION}

Hysterectomy is one of the most frequently performed surgical procedures in women worldwide. Although it was previously assumed that hysterectomy could have adverse effects on psychological well-being and might be associated with increased levels of depression, recent studies using a prospective design did not find evidence for decreased well-being or quality of life ${ }^{1,2}$ and even suggested that hysterectomy could reduce symptoms of depression and anxiety and increase general well-being. ${ }^{1,3}$ Despite overall good psychosocial recovery after hysterectomy, there might however be a subgroup of women reporting negative psychological outcomes. ${ }^{6}$ Several risk factors for suboptimal psychological recovery have been identified. These include pre-operative pain, ${ }^{7}$ pre-operative trait anxiety, ${ }^{7}$ previous emotional problems, ${ }^{5}$ and social support. ${ }^{8}$ Type of hysterectomy has generally been found to be unrelated to psychological outcome. ${ }^{3,4,9}$ However, it is still difficult to understand the interplay between these factors. Therefore, in line with the call for studies "to properly determine the psychological effects of hysterectomy", ${ }^{1}$ we examined the influence of sociodemographic, surgeryrelated, and pre-operative psychosocial variables, including childhood abuse, ${ }^{10}$ on depression, positive well-being, and vitality. In addition to our main outcomes depression, positive well-being, and vitality, we examined whether hysterectomy affects feelings of femininity. Concerns regarding femininity have previously been reported in women undergoing hysterectomy. ${ }^{5,11}$

The aim of this study is to assess the prevalence and predictors of adverse psychological outcome 3 and 12 months after elective hysterectomy.

\section{MATERIALS AND METHODS}

A prospective multicentre cohort study was performed in four Dutch hospitals: Maastricht University Medical Centre+, Catharina Hospital Eindhoven, Máxima Medical Centre Veldhoven, and Orbis Medical Centre Sittard-Geleen. This study was performed after approval by the Medical Ethics Committee of Maastricht UMC+, the Netherlands, and informed consent was given by all participants. Predictors of post-operative pain, physical, psychological, and sexual recovery were studied. In this article we focus on the multiple dimensions of psychological recovery. Clinical data on pain and physical recovery are published elsewhere. ${ }^{12}$ Data were collected during the week prior to surgery, and in the per-and postoperative period up to day 4. Long-term recovery was evaluated at 3 and 12 months after surgery. Preoperative and follow-up assessments were performed at home by postal questionnaires. Of all outcome measures also baseline measures were assessed. Patients recorded acute pain in a pain diary, data on surgery and anaesthesia were collected during surgery by the attending anaesthesiologist, gynaecologist, or study coordinator. 
Participants were scheduled for hysterectomy for benign indication between 2010 and 2014. Inclusion criteria were: informed consent, age 18-65 years, good understanding of the Dutch language, elective hysterectomy. Exclusion criteria were history of malignancy, illiteracy, and cognitive impairment.

Baseline data consisted of sociodemographic variables, preoperative health status including gynaecological history, comorbidity, ${ }^{13}$ physical functioning, ${ }^{14}$ and pain. ${ }^{15,} 16$ Collected surgery-related data were: type of hysterectomy (median lower abdominal or Pfannenstiel incision, vaginal approach, laparoscopic assisted vaginal hysterectomy [LAVH], laparoscopic hysterectomy [LH], total or subtotal, with or without oophorectomy), indication for surgery, duration of surgery, and type of anaesthesia (general anaesthesia [GA], GA combined with epidural, spinal, GA combined with spinal).

Psychological predictors were assessed using Dutch versions of several self-report questionnaires. For surgical fear the surgical fear questionnaire (SFQ) was used. ${ }^{17}$ Pain catastrophizing was assessed using the pain catastrophizing scale (PCS). ${ }^{18}$ Optimism/pessimism was assessed using the Life Orientation Test-revised (LOT-R). After reversal of the negative items a single optimism score was obtained. ${ }^{19}$ Trait affectivity was assessed using the positive and negative affect scale (PANAS). ${ }^{20}$ Social support was assessed using the sum score of the Medical Outcomes Study social support survey (MOS-SSS) and a one-item score reflecting the number of close friends/relatives available for social support. ${ }^{21}$ To screen for physical and sexual abuse during childhood two single items were included. ${ }^{22}$

For the outcome depression the Center for Epidemiological Studies-Depression (CES-D, range 0-60) scale was used. The numbers of prevalence/incidence are based on a previously established cut-off of $\geq 16 .^{23}$ For the multilevel predictor analysis the nondichotomised continuous scale was used. The outcome well-being was assessed using the well-being questionnaire (W-BQ12) energy (W-BQe) and positive well-being ( $W$ BQpw) subscales, range $0-12 .^{24}$ The predictor analyses for well-being were also performed using the continuous scales. In addition, participants were asked to what extent they felt less feminine after hysterectomy using an ordinal scale with four categories: not at all, a bit less, rather much less, very much less. Furthermore, it was assessed whether hysterectomy was experienced as a relief, a loss, or neutral. At baseline, corresponding expectations about feelings of femininity and hysterectomy were assessed. Finally, if patients underwent other surgery, or they reported a malignancy, or an event with potential major psychosocial impact during follow-up that might influence the answers of the follow-up questionnaires, they were excluded from follow-up analyses.

We planned to include 500 patients with an expected number of 300 suitable for follow-up analyses. ${ }^{12}$ Missing values on multi-item psychosocial questionnaires, physical functioning, and pain interference scale, if less than $20 \%$, were imputed by the participants mean score on that scale. Single-item sociodemographic and surgical variables were not imputed. Data are analysed using t-test, Chi-square or McNemar test if appropriate. To assess predictors for the 3 and 12 month follow-up outcomes, linear mixed 
model analyses with a random person intercept were performed. Based on an a priori selection a subset of pre-, per-, and postoperative variables was selected for multivariate analyses, to avoid overlap between variables with high level of similarity. This reduced the number of variables in the statistical models and additionally created more uniformity across analyses. To further reduce the number of psychological predictors the PCS and SFQ were combined into a single "surgical worries" score. ${ }^{12}$ Results on predictors are presented as estimate (SD). Model fit is shown by Akaike's Information Criterion, the lower its value the better the fit. See supporting information file S1 for further details of these analyses. All analyses were performed using IBM SPSS Statistics for Windows, version 23.0. Armonk, NY, USA.

\section{RESULTS}

Data of 419 participants were evaluated, see figure 1. Mean age (SD) was 46.9 (7.1) years, main indications for hysterectomy were menorrhagia/metrorrhagia (203 participants) and leiomyoma (136 participants). See table 1 and 2 for sociodemographic and surgery data.

The results on psychosocial baseline and follow-up data are presented in table 3 . The depression scores at 3 and 12 months showed small but significant improvements when compared to baseline mean (SD) of 10.9 (8.3). At 3 months the mean CES-D score decreased to 9.1 (8.5) ( $t: 4.410 ; d f: 401, p<0.001$ ) and at 12 months to 9.9 (8.8) (t: 2.280; $d f: 357, p=0.023)$. The prevalence of depression decreased from $23.7 \%$ at baseline to $19.1 \%$ at 3 months, and increased again to $21.4 \%$ at 12 months. However, changes were small and statistically non-significant. The incidence at 3 months was $8.2 \%$. The W-BQe scores revealed an increase from baseline to 3 months of mean (SD) 7.2 (2.7) to 7.7 (2.9) ( $t$ : $-2.983 ; d f: 401, p=0.003$ ). There was no evidence for a further mean increase, the score at 12 months was 7.9 (2.8). The mean W-BQpw scores at the three consecutive time points were 7.6 (3.0), 8.2 (3.2), and 8.0 (3.3) respectively. The mean increase from baseline to 3 months was statistically significant ( $t$ : $-3.480 ; d f: 399, p=0.001$ ), whereas the 12 month follow-up mean score was not significantly different from the mean at baseline.

Attitude towards hysterectomy was shown to be positive. After 3 months $98.3 \%$ of the participants experienced it as a relief or as neutral, whereas $91.6 \%$ indicated that they did not at all feel less feminine after hysterectomy. The results after 12 months were almost identical to the 3 months results, see table 3 . 


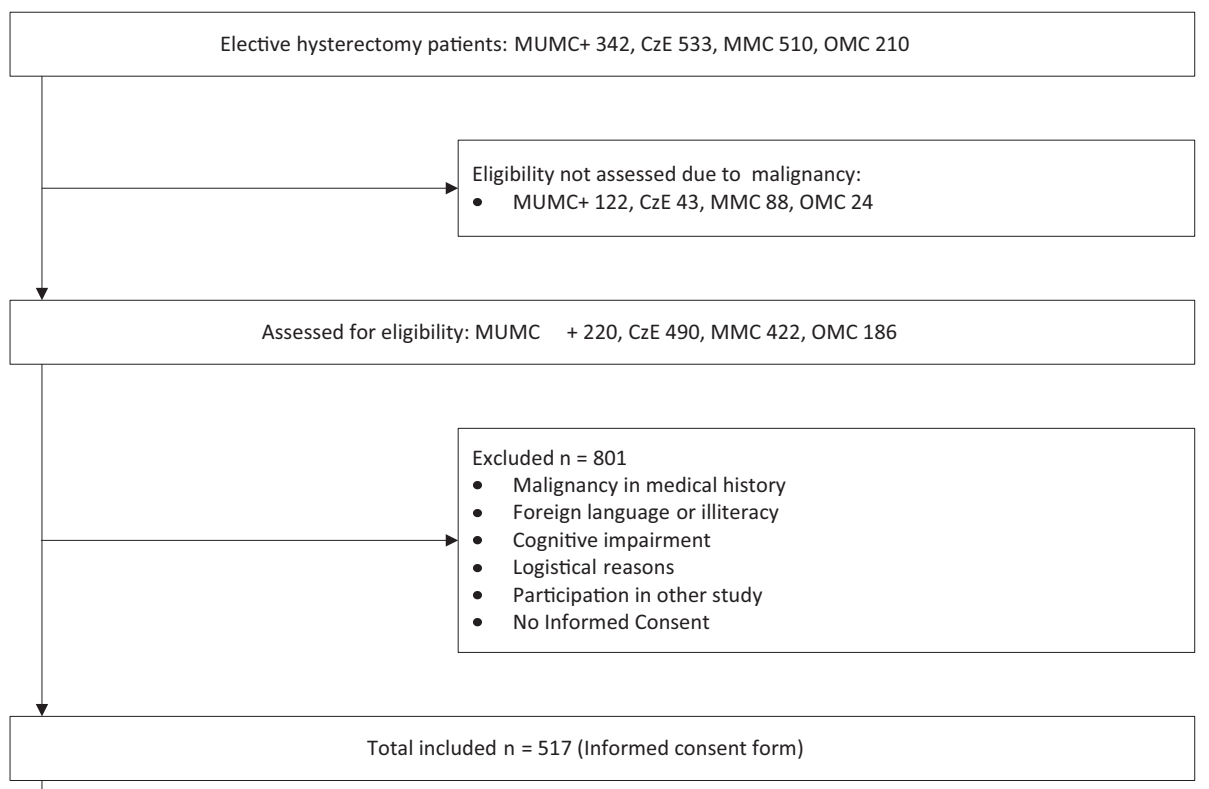

\begin{tabular}{|l|l|l|l|l}
\hline $\begin{array}{l}\text { Drop- out } n=31 \\
\text { Early withdrawal / no baseline data (17) } \\
\text { Hysterectomy performed elsewhere or cancelled (12) } \\
\text { Relaparotomy (2) } \\
\text { Inclusion error } n=18 \\
\text { Malignancy in medical history (14), age }>65 \text { (2), cognitive } \\
\text { impairment (2) }\end{array}$ \\
\hline
\end{tabular}

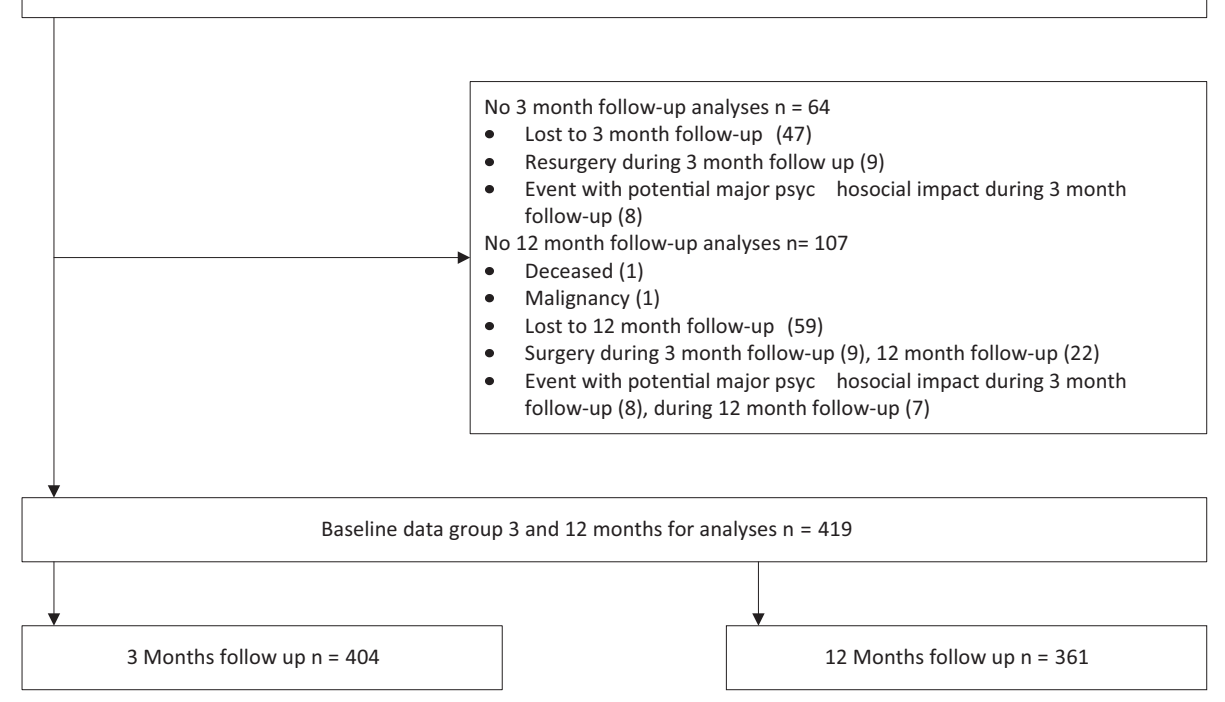

Figure 1. Patient flow 
Table 1. Baseline characteristics

\begin{tabular}{|c|c|}
\hline Measure & $N=419$ \\
\hline \multicolumn{2}{|l|}{ Centre } \\
\hline MUMC+ & $119(28 \%)$ \\
\hline CzE & $148(35 \%)$ \\
\hline MMC & $115(28 \%)$ \\
\hline OMC & $37(9 \%)$ \\
\hline Age & $46.9(7.1)$ \\
\hline Marital status: married / living together & $357(86 \%)[2]$ \\
\hline \multicolumn{2}{|l|}{ Education: } \\
\hline no / lower & $64(15 \%)$ \\
\hline intermediate & $245(59 \%)$ \\
\hline university / higher & $109(26 \%)$ \\
\hline missing data & 1 \\
\hline SF-36 physical activity (0-100) & $82.1(19.6)$ \\
\hline \multicolumn{2}{|l|}{ ASA: } \\
\hline I & $229(57 \%)$ \\
\hline$\|$ & $165(41 \%)$ \\
\hline III & $7(2 \%)$ \\
\hline missing data & 18 \\
\hline BMI & $26.5(4.9)$ \\
\hline \multicolumn{2}{|l|}{ Highest pain last week (0-10): } \\
\hline related to planned hysterectomy & $3(0-7)$ \\
\hline non-related to planned hysterectomy & $0(0-6)$ \\
\hline \multicolumn{2}{|l|}{ Gynaecological history: } \\
\hline hysterectomy-related surgery last year & $74(18 \%)[4]$ \\
\hline pre-menopausal status & $317(77 \%)[8]$ \\
\hline HRT & $15(4 \%)[3]$ \\
\hline contraception use & $86(21 \%)[3]$ \\
\hline parity & $1.8(1.1)$ \\
\hline vaginal or caesarean delivery & $343(83 \%)[6]$ \\
\hline caesarean section & $60(14 \%)[2]$ \\
\hline sexually active & $333(83 \%)[16]$ \\
\hline
\end{tabular}

MUMC+: Maastricht University Medical Centre+; CzE: Catharina hospital Eindhoven; MMC: Máxima Medical Centre, Veldhoven; OMC: Orbis Medical Centre, Sittard-Geleen.

Numbers are mean (SD), median $\left(25^{\text {th }}-75^{\text {th }}\right.$ percentile), or number (\%), [missing data].

SF-36 physical activity: Rand health survey short-form 36 physical activity subscale; ASA: American Society of Anaesthesiologists; BMI: body mass index. Hysterectomy-related surgery last year: answer yes to "If you had surgery last year, was this related to the planned hysterectomy?" HRT: hormonal replacement therapy. 


\section{Chapter 6}

Table 2. Surgery data

\begin{tabular}{|c|c|}
\hline Measure & $N=419$ \\
\hline \multicolumn{2}{|l|}{ Indication (more than 1 possible): } \\
\hline leiomyoma & $136(33 \%)$ \\
\hline prolapse & $71(17 \%)$ \\
\hline menorrhagia / metrorrhagia & $203(48 \%)$ \\
\hline dysmenorrhoea & $26(6 \%)$ \\
\hline endometriosis / adenomyosis & $23(6 \%)$ \\
\hline abdominal pain & $26(6 \%)$ \\
\hline cervical dysplasia & $24(6 \%)$ \\
\hline other & $25(6 \%)$ \\
\hline \multicolumn{2}{|l|}{ Anaesthesia: } \\
\hline GA & $351(84 \%)$ \\
\hline Spinal / GA \& epidural / GA \& spinal & $68(16 \%)$ \\
\hline \multicolumn{2}{|l|}{ Incision: } \\
\hline median lower abdominal & $22(5 \%)$ \\
\hline Pfannenstiel & $50(12 \%)$ \\
\hline vaginal & $170(41 \%)$ \\
\hline $\mathrm{LH}$ & $87(21 \%)$ \\
\hline LAVH & $90(21 \%)$ \\
\hline \multicolumn{2}{|l|}{ Hysterectomy: } \\
\hline total hysterectomy & $393(95 \%)[3]$ \\
\hline oophorectomy unilateral & $13(3 \%)$ \\
\hline oophorectomy bilateral & $16(4 \%)$ \\
\hline prolapse surgery & 75 (19\%) [22] \\
\hline duration (hrs) & $1: 48(0: 51)$ \\
\hline Blood loss (ml) & $282(373)$ \\
\hline
\end{tabular}

Numbers are mean (SD), median $\left(25^{\text {th }}-75^{\text {th }}\right.$ percentile), or number (\%), [missing data].

GA: general anaesthesia. Hysterectomy total: compared with subtotal hysterectomy. LH: laparoscopic hysterectomy, LAVH: laparoscopic assisted vaginal hysterectomy. 
Table 3. Psychosocial measures at baseline, 3 and 12 months after hysterectomy

\begin{tabular}{|c|c|c|c|}
\hline \multirow[t]{2}{*}{ Measure } & $\begin{array}{l}\text { Baseline } \\
\text { Sample }\end{array}$ & $\begin{array}{l}\text { Sample } 3 \text { month } \\
\text { follow-up }\end{array}$ & $\begin{array}{l}\text { Sample } 12 \text { month } \\
\text { follow-up }\end{array}$ \\
\hline & $N=419$ & $N=404$ & $N=361$ \\
\hline CES-D (0-60) & $10.9(8.3)$ & $9.1(8.5)^{* * *}$ & $9.9(8.8)^{*}$ \\
\hline W-BQ energy (0-12) & $7.2(2.7)$ & $7.7(2.9)^{* *}$ & $7.9(2.8)^{* * *}$ \\
\hline W-BQ positive well-being (0-12) & $7.6(3.0)$ & $8.2(3.2)^{* *}$ & $8.0(3.3)$ \\
\hline \multicolumn{4}{|l|}{ Experience hysterectomy: } \\
\hline relief & $314(76 \%)$ & $271(67 \%)^{* *}$ & $247(70 \%)$ \\
\hline neutral & $88(22 \%)$ & $124(31 \%)$ & $100(28 \%)$ \\
\hline loss & $10(2 \%)$ & $7(2 \%)$ & $8(2 \%)$ \\
\hline missing data & 7 & 2 & 6 \\
\hline \multicolumn{4}{|l|}{ Experience less femininity: } \\
\hline not at all & $347(84 \%)$ & $369(92 \%)^{* * *}$ & $331(92 \%)^{* * *}$ \\
\hline a bit less & $57(14 \%)$ & $28(7 \%)$ & $23(6 \%)$ \\
\hline rather much less & $4(1 \%)$ & $3(<1 \%)$ & $2(1 \%)$ \\
\hline very much less & $6(1 \%)$ & $3(<1 \%)$ & $3(1 \%)$ \\
\hline missing data & 5 & 1 & 2 \\
\hline SFQ short-term (0-40) & $16.1(9.9)$ & - & - \\
\hline SFQ long-term (0-40) & $9.4(8.6)$ & - & - \\
\hline PCS (0-52) & $12.3(8.2)$ & - & - \\
\hline LOT-R (0-24) & $17.0(3.7)$ & - & - \\
\hline PANAS positive (5-25) & $17.5(3.2)$ & - & - \\
\hline PANAS negative (5-25) & $10.2(4.2)$ & - & - \\
\hline MOS-SSS number of persons & $9.9(6.9)$ & - & - \\
\hline MOS-SSS total $(0-100)$ & $85.8(18.6)$ & - & - \\
\hline History of physical abuse & $50(12 \%)[3]$ & - & - \\
\hline History of sexual abuse & $80(19 \%)[7]$ & - & - \\
\hline History of psychological problems & $120(29 \%)[3]$ & & \\
\hline Expected pain after 4 days $(0-10)$ & $5(3-6)$ & - & - \\
\hline
\end{tabular}

Depression: Center for Epidemiological Studies-Depression (CES-D) scale. W-BQ: well-being questionnaire, positive well-being and energy subscales.

Experience hysterectomy and femininity at baseline: expected feelings after hysterectomy; SFQ: surgical fear questionnaire; PCS: pain catastrophizing scale; LOT-R: life orientation test-revised; PANAS: positive and negative affect scale; MOS-SSS: Medical Outcomes Study-social support survey with subscales tangible support, emotional/informative support, affective support, and positive social interaction. History of psychological problems: answer yes to "Did you ever suffer from psychological problems for which you needed support?" Numbers are mean (SD), median $\left(25^{\text {th }}-75^{\text {th }}\right.$ percentile), or number (\%), [missing data].

Significant difference compared with baseline: ${ }^{*} p<0.05,{ }^{*} p<0.01, * * * p<0.001$ (for Experience less femininity: dichotomized, not at all versus bit less/rather/very much less).

An overview of all bivariate significant predictors for depression and well-being is presented in the results section of the supporting information, file S2. The results of linear mixed analyses for depression are shown in table 4, left column (only predictors significant at $p<0.05$ level are shown). Higher baseline depression score and non-hysterectomy-related baseline pain were associated to elevated depression scores at follow-up. Furthermore, increased perioperative blood loss was associated with lower depression scores at 3 
months and, to a lesser extent, at 12 months. Finally, for women that reported an infection at 12 month follow-up it appeared that mean depression scores increased more strongly from 3 to 12 months compared to women that did not report an infection.

The results for the well-being energy subscale are presented in table 4 second column. Increased baseline W-BQe and social support predicted higher W-BQe scores whereas baseline pain, high negative affect scores, and pain at the post anaesthesia care unit (PACU) predicted lower W-BQe scores. There were also several interaction effects with time. For postmenopausal women mean W-BQe scores were higher than for premenopausal women at 3 months but not at 12 months. Contraception use at baseline decreased $\mathrm{W}$-BQe at 3 months and to a lesser extent at 12 months. Indication of cervical dysplasia (compared to leiomyoma) decreased W-BQe at 3 months and to a lesser extent at 12 months.

Table 4. Predictors of depression and well-being 3 and 12 months after hysterectomy

\begin{tabular}{|c|c|c|c|c|c|c|}
\hline \multirow[t]{2}{*}{ Predictors } & \multicolumn{2}{|l|}{$\begin{array}{l}\text { Depression } \\
\mathrm{N}=353\end{array}$} & \multicolumn{2}{|l|}{$\begin{array}{l}\text { Well-Being } \mathrm{E} \\
\mathrm{N}=305\end{array}$} & \multicolumn{2}{|l|}{$\begin{array}{l}\text { Well-Being PW } \\
N=404\end{array}$} \\
\hline & Est (SD) & $P$ & Est (SD) & $P$ & Est (SD) & $P$ \\
\hline \multicolumn{7}{|l|}{ Time } \\
\hline 3 months & Reference & & Reference & & Reference & \\
\hline 12 months & $0.516(0.908)$ & 0.570 & $0.643(0.585)$ & 0.273 & $-2.703(0.835)$ & 0.001 \\
\hline \multicolumn{7}{|l|}{ Menopausal status } \\
\hline pre-menopausal & - & & Reference & & - & \\
\hline post-menopausal & - & & $1.088(0.648)$ & 0.094 & - & \\
\hline Contraception use & - & & $-1.977(0.667)$ & 0.003 & - & \\
\hline \multicolumn{7}{|l|}{ Indication } \\
\hline leiomyoma & Reference & & Reference & & Reference & \\
\hline prolapse & $0.575(2.075)$ & 0.782 & $-1.572(0.812)$ & 0.053 & $-1.337(0.783)$ & 0.088 \\
\hline abdominal pain & $-4.200(2.738)$ & 0.126 & $0.730(1.222)$ & 0.516 & $1.484(1.058)$ & 0.162 \\
\hline cervical dysplasia & 4.941 (3.095) & 0.111 & $-3.451(1.230)$ & 0.005 & $-1.500(1.193)$ & 0.209 \\
\hline Depression (0-60) & $0.623(0.041)$ & $<0.001$ & - & & - & \\
\hline Well-being energy (0-12) & - & & $0.391(0.052)$ & $<0.001$ & - & \\
\hline Well-being positive well-being $(0-12)$ & - & & - & & $0.371(0.048)$ & $<0.001$ \\
\hline Negative affect (5-25) & & & $-0.096(0.033)$ & 0.003 & - & \\
\hline Surgery-related worries & - & & - & & $-0.369(0.127)$ & 0.004 \\
\hline Optimism (0-24) & - & & - & & $-0.057(0.073)$ & 0.438 \\
\hline Social support (0-100) & - & & $0.019(0.007)$ & 0.006 & $0.013(0.007)$ & 0.063 \\
\hline $\begin{array}{l}\text { Non-hysterectomy-related baseline } \\
\text { pain }(0-10)\end{array}$ & $0.306(0.101)$ & 0.003 & $-0.089(0.040)$ & 0.026 & $-0.101(0.038)$ & 0.008 \\
\hline Blood loss (ml) & $-0.005(0.002)$ & 0.013 & - & & - & \\
\hline Pain at PACU $(0-10)$ & - & & $-0.134(0.049)$ & 0.007 & - & \\
\hline Surgery-related infection 12 month & $-6.253(3.490)$ & 0.074 & $2.327(1.362)$ & 0.088 & - & \\
\hline \multicolumn{7}{|l|}{ Interaction with time } \\
\hline postmenopausal status & - & & $-0.833(0.372)$ & 0.026 & - & \\
\hline contraception use & - & & $1.017(0.387)$ & 0.009 & - & \\
\hline indication abdominal pain & $2.934(1.593)$ & 0.066 & - & & $-1.395(0.643)$ & 0.031 \\
\hline
\end{tabular}




\begin{tabular}{|c|c|c|c|c|c|c|}
\hline \multirow[t]{3}{*}{ Predictors } & \multirow{2}{*}{\multicolumn{2}{|c|}{$\begin{array}{l}\text { Depression } \\
\mathrm{N}=353\end{array}$}} & \multirow{2}{*}{\multicolumn{2}{|c|}{$\begin{array}{l}\text { Well-Being } E \\
N=305\end{array}$}} & \multirow{2}{*}{\multicolumn{2}{|c|}{$\begin{array}{l}\text { Well-Being PW } \\
N=404\end{array}$}} \\
\hline & & & & & & \\
\hline & Est (SD) & $P$ & Est (SD) & $P$ & Est (SD) & $P$ \\
\hline indication cervical dysplasia & - & & $1.562(0.706)$ & 0.028 & - & \\
\hline optimism & - & & - & & $0.136(0.044)$ & 0.002 \\
\hline blood loss & $0.003(0.001)$ & 0.011 & - & & - & \\
\hline surgery-related infection 12 month & $4.276(1.994)$ & 0.033 & $-2.121(0.778)$ & 0.007 & - & \\
\hline Akaike's Information Criterion & 4485.4 & & 2693.6 & & 3491.9 & \\
\hline
\end{tabular}

Depression: CES-D, Center for Epidemiological Studies - Depression (0-60); Well-being: W-BQ, well-being questionnaire, well-being $\mathrm{E}=$ energy and well-being $\mathrm{PW}=$ positive well-being subscale $(0-12)$.

Numbers are estimates (SD) and $p$ value. Significant $p$ value in bold. Main effects and interaction with time, i.e. an additional effect on 12 month compared with 3 month outcome, are only shown for variables with $p<0.05$.

Surgery-related worries: range -1.675 to 3.665 , is based on regression scores, resulting of factor analysis performed on the SFQ, PCS, LOT-R, CES-D and W-BQ12. Two factors revealed, the first, consisting of the SFQ and PCS named surgery-related worries. Negative affect: PANAS, Optimism: LOT-R (0-24), Social support: MOS-SSS (0-100), Expected pain: expected pain after 4 days (NRS 0-10), PACU: post anaesthesia care unit. Surgery-related infection: self-reported surgery-related infection during 3 or 12 month follow-up.

The Akaike's Information Criterion of all three outcome measures improved when compared with the model with control variables, baseline reference, and time: depression $\mathrm{AIC}=5025.6, \mathrm{~W}-\mathrm{BQ}$ e 3520.6, and W-BQpw 3664.8 .

For positive well-being, the preoperative factors lower baseline W-BQpw, nonhysterectomy-related baseline pain, and surgery-related worries predicted lower WBQpw. Two interaction effects with time were found. In women with indication abdominal pain the decrease in mean W-BQpw from 3 to 12 months was stronger than for women with indication leiomyoma. Baseline optimism did not appear to be associated to W-BQpw at 3 months, but at 12 months this relation was significantly more positive. Of the per- and postoperative variables, none remained in the model. See table 4 third column.

\section{COMMENT}

The results of this study showed that hysterectomy was related to slightly increased wellbeing and decreased depression. Universal predictors for both a higher level of depression and a lower level of well-being 3 and 12 months after hysterectomy were corresponding baseline levels and baseline pain non-related to the planned hysterectomy. The course of depression and well-being energy was affected by self-reported postoperative infection. Several other gynaecological or psychological predictors additionally predicted depression or well-being outcomes.

Strength of this study is that use was made of extensive questionnaire packages, allowing us to assess multiple sociodemographic, gynaecological, psychosocial, and surgical predictors of depression and well-being. We were able to explore whether previous 
findings were supported, and simultaneously explore other factors that may be involved in psychological recovery after hysterectomy. Furthermore, the analyses were performed including the baseline measure of the outcome measures. This enabled us to direct the selection of predictors towards variables predicting change in depression or well-being caused by hysterectomy, rather than predicting a general level of depression and well-being. A limitation is that depression was not clinically diagnosed. Furthermore, the use of hormonal replacement therapy was assessed only at baseline, not at follow-up. This may bias our findings with regard to the predictor contraceptive use. Finally, the exploration of a large number of baseline data for statistical predictor analyses increases the risk of making Type I errors. This should be considered when drawing conclusions from our study. On the other hand, our findings with regard to the outcome depression are in agreement with a recent meta-analysis, reporting that depressive symptoms might improve after hysterectomy, and that the presence of depressive symptoms preoperatively is a risk factor for postoperative depression. ${ }^{1}$ One of the preoperative factors that was related to changes in depression at follow-up was preoperative pain. This association was already described in previous studies. ${ }^{7,25}$ Furthermore postoperative infection was related to changes in depression at follow-up. This finding is complementary with publications stipulating evidence for the link between depression and inflammatory and immunological processes. ${ }^{26,27}$ In contrast, subgroup analyses in the meta-analyses of Darwish et al. ${ }^{1}$ indicated that the decrease in depression after hysterectomy was mainly found in studies including premenopausal women only whereas studies that involved postmenopausal women or women with oophorectomy did not find a significant decrease in depression. In our study no effects of menopausal status or bilateral oophorectomy on depression were found. Except for baseline depression scores, none of the other psychological variables were predictive of depression scores at 3 and 12 months in our study. This is in contrast to previous studies that did report that pre-operative psychological status predicted depression at follow-up. ${ }^{5,7}$, 25,28 Concerning the outcome well-being, we found that several baseline psychological factors were predictive of outcome levels. Post-operative energy levels were lower in the presence of negative affect and higher when social support was available. This confirms a previous report where negative affect predicted poorer outcome on all EQ-5D dimensions 12 months after percutaneous coronary interventions. ${ }^{29}$ Moreover, social support has been related to better quality of life in other health settings, e.g. in stroke patients. ${ }^{30}$ Finally, higher levels of positive well-being at follow-up were positively associated with optimism and negatively with surgical worries. Optimism has been related to better well-being and higher quality of life after surgery across a range of different procedures. ${ }^{31}$ In our previous report of the physical dimensions of recovery in the same patient group, we found surgical worries to be related to increased long-term pain and decreased feelings of global recovery. ${ }^{12}$ Together with the results of the present analyses it seems that worrying about the upcoming surgery has a relatively pervasive effect on a large range of outcomes. Similar to all other outcomes in this cohort study, ${ }^{12}$ also 
postoperative well-being was negatively affected by pre- and acute postoperative pain. Several other studies assessed well-being after hysterectomy, however these studies compared different interventions, and did not provide results on the impact of perioperative pain on well-being. 3, 4, 32, 33 Nonetheless, these studies support our results concerning type of hysterectomy, which did not affect well-being. We found that postmenopausal status was associated with higher W-BQe at 3 months, an effect that seemed to disappear at 12 months. This could possibly mean that the potential negative influence of perimenopausal status in general on the overall energy status can aggravate more in the postoperative phase in those women stopping exogenous female hormones.

Rather positive findings are that the majority of the patients indicated that they experienced hysterectomy as a relief and that feelings of femininity were not adversely affected. A previously performed literature review ${ }^{6}$ reported inconclusive evidence with regard to effects of hysterectomy on body image.

Altogether, this study and many studies performed earlier reveal the potential impact of poor psychosocial preoperative status. Therefore selection of patients at increased preoperative risk and establishment of effective psychological interventions deserve more attention. ${ }^{34}$

This study revealed that apart from baseline level of depression or well-being, preoperative psychosocial status, perioperative pain and postoperative infection are predictors of psychological outcome after hysterectomy. For the majority of patients hysterectomy leads to small but significant improvements in postoperative depression and well-being. Hysterectomy seems not to adversely affect feelings of femininity. To further improve postoperative outcome, a focus on analgesic treatment and infection control might benefit patients' postoperative psychological health.

\section{ACKNOWLEDGEMENTS}

We thank A. van Ommering (MMC Veldhoven) for the effective patient recruitment and data collection, and S. van Riet (Maastricht University) for her support with data logistics. Furthermore we thank dr. M. Bergmans for facilitating the data collection of two patients included at Maastricht UMC+ who had their surgery at the Laurentius hospital Roermond. Finally we thank M. Willemsen, L. Muntinga, N.A.M. Oversier, R.V.G. Soekhoe, B. Vreeswijk, M. Herman, and B.A. de Waal, for their contribution to patient inclusion and data collection during their traineeship or residentship. 


\section{REFERENCES}

1. Darwish M, Atlantis E, Mohamed-Taysir T Psychological outcomes after hysterectomy for benign conditions: a systematic review and meta-analysis. Eur J Obstet Gynecol Reprod Biol. 2014; 174: 5-19.

2. Khastgir G, Studd JW, Catalan J The psychological outcome of hysterectomy. Gynecol Endocrinol. 2000; 14(2): 132-141.

3. Aziz A, Bergquist C, Nordholm L, et al. Prophylactic oophorectomy at elective hysterectomy. Effects on psychological well-being at 1-year follow-up and its correlations to sexuality. Maturitas. 2005; 51(4): 349357.

4. Persson P, Brynhildsen J, Kjolhede P, et al. A 1-year follow up of psychological wellbeing after subtotal and total hysterectomy--a randomised study. BJOG. 2010; 117(4): 479-487.

5. Yen JY, Chen YH, Long CY, et al. Risk factors for major depressive disorder and the psychological impact of hysterectomy: a prospective investigation. Psychosomatics. 2008; 49(2): 137-142.

6. Flory N, Bissonnette F, Binik YM Psychosocial effects of hysterectomy: literature review. J Psychosom Res. 2005; 59(3): 117-129.

7. Vandyk AD, Brenner I, Tranmer J, et al. Depressive symptoms before and after elective hysterectomy. J Obstet Gynecol Neonatal Nurs. 2011; 40(5): 566-576.

8. Thornton EW, McQueen C, Rosser R, et al. A prospective study of changes in negative mood states of women undergoing surgical hysterectomy: the relationship to cognitive predisposition and familial support. J Psychosom Obstet Gynaecol. 1997; 18(1): 22-30.

9. Rohl J, Kjerulff K, Langenberg P, et al. Bilateral oophorectomy and depressive symptoms 12 months after hysterectomy. Am J Obstet Gynecol. 2008; 199(1): 22 e21-25.

10. Salmon P, Hill J, Krespi R, et al. The role of child abuse and age in vulnerability to emotional problems after surgery for breast cancer. Eur J Cancer. 2006; 42(15): 2517-2523.

11. Ferroni P, Deeble J Women's subjective experience of hysterectomy. Aust Health Rev. 1996; 19(2): 4055.

12. Theunissen M, Peters ML, Schepers J, et al. Recovery 3 and 12 months after hysterectomy: epidemiology and predictors of chronic pain, physical functioning, and global surgical recovery. Medicine (Baltimore). 2016; 95(26): e3980.

13. Berg van den J, Wulp van der C, Rapport van de Werkgroep Revisie POLS-Gezondheidsenquête 1999. 2003, Statistics Netherlands (CBS): Heerlen.

14. Ware JE, Jr., Sherbourne CD The MOS 36-item short-form health survey (SF-36). I. Conceptual framework and item selection. Medical care. 1992; 30(6): 473-483.

15. Cleeland CS, Ryan KM Pain assessment: global use of the Brief Pain Inventory. Ann Acad Med Singapore. 1994; 23(2): 129-138.

16. van Seventer R, Vos C, Giezeman M, et al. Validation of the Dutch version of the DN4 diagnostic questionnaire for neuropathic pain. Pain Pract. 2013; 13(5): 390-398.

17. Theunissen M, Peters ML, Schouten EG, et al. Validation of the Surgical Fear Questionnaire in Adult Patients Waiting for Elective Surgery. PLoS One. 2014; 9(6): e100225.

18. Sullivan MJL, Bishop SR, Pivik J The Pain Catastrophizing Scale: Development and validation. Psychol Assessment. 1995; 7(4): 524-532.

19. Scheier MF, Carver CS, Bridges MW Distinguishing optimism from neuroticism (and trait anxiety, selfmastery, and self-esteem): A reevaluation of the Life Orientation Test. J Pers Soc Psychol. 1994; 67(6): 1063-1078.

20. Mackinnon A, Jorm AF, Christensen $\mathrm{H}$, et al. A short form of the Positive and Negative Affect Schedule: Evaluation of factorial validity and invariance across demographic variables in a community sample. Pers Indiv Differ. 1999; 27(3): 405-416.

21. Sherbourne CD, Stewart AL The MOS social support survey. Soc Sci Med. 1991; 32(6): 705-714.

22. Thombs BD, Bernstein DP, Ziegelstein RC, et al. A brief two-item screener for detecting a history of physical or sexual abuse in childhood. Gen Hosp Psychiatry. 2007; 29(1): 8-13. 
23. Radloff LS The CES-D Scale: A self-report depression scale for research in the general population. Appl Psych Meas. 1977; 1(3): 385-401.

24. Bradley C The 12-Item Well-Being Questionnaire: origins, current stage of development, and availability. Diabetes Care. 2000; 23(6): 875.

25. Ewalds-Kvist SB, Hirvonen T, Kvist M, et al. Depression, anxiety, hostility and hysterectomy. J Psychosom Obstet Gynaecol. 2005; 26(3): 193-204.

26. Ghoneim MM, O'Hara MW Depression and postoperative complications: an overview. BMC Surg. 2016; 16(1): 5 .

27. Maes M Depression is an inflammatory disease, but cell-mediated immune activation is the key component of depression. Prog Neuropsychopharmacol Biol Psychiatry. 2011; 35(3): 664-675.

28. Donoghue AP, Jackson HJ, Pagano R Understanding pre- and post-hysterectomy levels of negative affect: a stress moderation model approach. J Psychosom Obstet Gynaecol. 2003; 24(2): 99-109.

29. Versteeg H, Pedersen SS, Erdman RA, et al. Negative and positive affect are independently associated with patient-reported health status following percutaneous coronary intervention. Qual Life Res. 2009; 18(8): 953-960.

30. Kruithof WJ, van Mierlo ML, Visser-Meily JM, et al. Associations between social support and stroke survivors' health-related quality of life--a systematic review. Patient Educ Couns. 2013; 93(2): 169-176.

31. Carver CS, Scheier MF, Segerstrom SC Optimism. Clin Psychol Rev. 2010; 30(7): 879-889.

32. Farquhar CM, Harvey SA, Yu Y, et al. A prospective study of 3 years of outcomes after hysterectomy with and without oophorectomy. AJOG. 2006 194, : 711-717.

33. Persson P, Wijma K, Hammar M, et al. Psychological wellbeing after laparoscopic and abdominal hysterectomy--a randomised controlled multicentre study. BJOG. 2006; 113(9): 1023-1030.

34. Shehmar M, Gupta JK The influence of psychological factors on recovery from hysterectomy. J R Soc Med. 2010; 103(2): 56-59. 


\section{SUPPORTING INFORMATION FILES}

\section{S1. Variable selection and data reduction: Methods}

Sociodemographic data, baseline health, gynaecological history, psychosocial status, perioperative data on surgery, anaesthesia and acute pain, and self-reported infection at follow-up were assessed as predictors of psychological recovery. Based on an a priori selection a subset of predictors was selected for multivariate analyses to avoid overlap between variables with high level of similarity. This reduced the number of variables in the statistical models and additionally created more uniformity across analyses. ASAclassification was selected as a proxy for general preoperative health status, number of active days per week (minimum of 30 minutes of physical activity), SF-36 physical functioning, and history of chronic disease. Baseline pain was analysed using highest numeric rating score (NRS) of pain related or not related to the planned hysterectomy. Acute postsurgical pain was analysed using the pain score (NRS) at the post anaesthesia care unit (PACU) and neuropathic pain (NPP) at postoperative day 4. Self-reported surgeryrelated infection was assessed as postoperative complication. The social support variable number of close friends/relatives was omitted because, covering a broad range of social support dimensions, the MOS-SSS total score was preferred for multivariate analyses. Furthermore, type of incision was dichotomised as laparotomy versus vaginal/LH/LAVH for the multivariate analyses and type of anaesthesia was dichotomised as GA versus other (including combination of types). Oophorectomy was dichotomised as bilateral versus no/unilateral, to enable assessment of potential hormonal effects on depression and well-being at follow-up.

Linear mixed model analyses with a random person intercept were performed. The impact of control variables participating hospital, age, type of incision (dichotomised), and furthermore the baseline measure of the outcome, time, somatic and psychological preoperative predictors, and the per- and postoperative variables was assessed. The control variables, baseline measure, and time were entered in the equation using a forced entry method. All other predictors were selected using a backward deletion procedure with $p<0.10$ as decision criterion. A final significance level of alpha $=0.05$ was used.

To further reduce the load of bivariate significant predictors on the multivariate model an in-between step was made. Based on domain or point in time predictors were clustered in six blocks. Block 1 consisted of the control variables hospital, age, type of incision, and furthermore of the baseline measure of the outcome, and a time variable defining 3 and 12 month follow-up. Block 2 consisted of predictors concerning baseline health status and sociodemographic variables, block 3 consisted of gynaecological history and indication for surgery, block 4 concerned psychosocial predictors, block 5 contained predictors on surgery and acute pain, and block 6 represented self-reported infection during follow-up. Bivariate significant predictors of each of the blocks 2-6 were added per block separately to the control variables of block 1 , and backward analyses 
were applied to select which variables of each block were selected (criterion $p<0.10$ ) for the final multivariate analyses. A truncated version only showing predictors significant at $p<0.05$ level is presented in the manuscript. The presented estimates (SD) reflect the change in outcome for each unit of change in the predictor. Multicollinearity was assessed by evaluating the variance inflation factor and tolerance obtained by repeating the multivariate analyses in a linear regression model.

\section{S2. Variable selection and data reduction: Results}

Predictors of psychological recovery after 3 months.

Depression - bivariate significant predictors, decision criterion $p<0.10$.

Besides the control variables hospital, age, type of incision, other variables entered in the multivariate model were baseline depression and the time variable, education level, hysterectomy-related surgery last year, contraception use, indication for surgery, surgery-related worries, social support (MOS-SSS), baseline pain non-related to the planned hysterectomy, type of anaesthesia, total versus subtotal hysterectomy, blood loss, and infection reported at 12 months. Significant but not entered in multivariate analyses because of our a priori selection or use in the aggregate score surgery-related worries, were baseline SF-36 physical functioning and PCS.

Well-being energy - bivariate significant predictors, decision criterion $p<0.10$.

Of the control variables only type of incision was significant including an interaction effect with the time variable. The variables that were used in the multivariate analyses were baseline $\mathrm{W}$-BQe, time by incision interaction variable, baseline pain non-related to the planned hysterectomy, menopausal state, contraception use, indication for surgery, expectations on hysterectomy (relief/neutral/loss), negative affect, social support, surgery-related worries, expected pain at postoperative day 4, type of anaesthesia, pain at the PACU, NPP at postoperative day 4, and infection at 12 month follow-up. Not entered in multivariate analyses because of our a priori selection or use in the aggregate score surgery-related worries, were number of active days/week, SFQ-S, SFQ-I, CES-D, and W-BQpw (CES-D, and W-BQpw both serving as baseline for the corresponding outcome), non-hysterectomy-related NPP at baseline, and highest pain NRS at postoperative day 4.

Positive well-being - bivariate significant predictors, decision criterion $p<0.10$.

In addition to the control variables, baseline level of W-BQpw, and time, the variables ASA, indication, optimism, negative affect, social support, history of physical abuse, surgery-related worries, baseline pain, non-related to the planned hysterectomy, performance of prolapse surgery, and NPP at postoperative day 4 were used in multivariate analyses. Depression was also associated with W-BQpw but not entered in multivariate analyses because of our a priori selection: it was only used for baseline correction of outcome depression. Other variables excluded from analyses because of our a priori selection were chronic disease yes/no, history of psychological problems yes/no, expected number of days until full reuptake of normal activities, baseline non- 
Chapter 6

hysterectomy-related NPP, and analgesia use at postoperative day 4. SFQ-S, SFQ-I, nor PCS were significant in bivariate analyses, however, surgery-related worries was, and therefore included in the model.

Multicollinearity played no role for any of the three outcome measures. 
Chapter

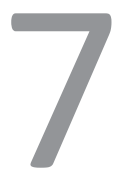

General discussion 


\section{OUTLINE}

This dissertation reflects on two aspects related to postoperative quality of life.

The first is surgical fear: the impact of surgical fear, or anxiety, and catastrophizing on postoperative quality of life in terms of chronic postsurgical pain is assessed in a literature review and meta-analysis. To improve research in this area the validation of a new instrument for the assessment of preoperative fear, the Surgical Fear Questionnaire (SFQ) is described.

The second aspect consists of the study on predictors and outcomes of postoperative quality of life in a homogenous group of patients. In addition to surgical fear other psychosocial and medical predictors are assessed in a cohort of women undergoing a hysterectomy. A description is given of postoperative quality of life in terms of physical and psychological recovery after 3 and 12 months. A summary of the main findings is given below, followed by a discussion.

\section{Main results of the studies}

There is evidence that anxiety and catastrophizing play a role in the development of chronic postsurgical pain (CPSP). (Chapter 2)

The SFQ is a valid and reliable eight-item index of surgical fear, consisting of two subscales: fear of the short-term consequences of surgery and fear of the long-term consequences. (Chapter 3, 4)

The SFQ is sensitive to small changes in pre-operative fear before cataract surgery. Pre-operative pain and stressful life situations are related to higher levels of surgical fear. Except for certain subgroups based on for example educational level and antidepressant use, the observed variations in fear scores were clinically insignificant, suggesting that in general, surgical fear scores assessed at different time points in the week before surgery are well comparable. (Chapter 4)

Several predictors were identified for CPSP, physical functioning, and global surgical recovery after hysterectomy. CPSP was predicted by preoperative pain, surgery-related worries, acute postsurgical pain, and surgery-related infection. Baseline level of physical functioning, participating hospital, general psychological robustness, indication for surgery, acute postsurgical pain, and surgery-related infection were significant predictors of physical functioning. Predictors of global surgical recovery were baseline expectations, surgery-related worries, ASA-classification, type of anesthesia, acute postsurgical pain, and surgery-related infection. Some of the identified factors are modifiable and optimization of patients' preoperative pain status and psychological condition as well as reduction of acute postsurgical pain and surgery-related infection may lead to improvement of outcome. (Chapter 5)

Perioperative pain and postoperative infection predict changes in psychological status after hysterectomy. (Chapter 6 ) 
Most women report to have recovered adequately from their hysterectomy. For the majority of patients hysterectomy-related pain decreased significantly from pre- to postoperative, small increases in physical function were found, a high level of global surgical recovery was reported, resumption of daily activities was as expected, and hysterectomy led to small but significant improvements on postoperative depression and well-being. Hysterectomy seemed not to adversely affect feelings of femininity. (Chapter 5, 6)

\section{POSTOPERATIVE QUALITY OF LIFE: SURGICAL FEAR AND OTHER PREDICTORS}

The predictive value of fear for CPSP: a review of the existing literature

This thesis started with a literature study on a well-recognizable psychological issue, surgical fear. The research question was:

"What is the current level of evidence concerning the hypothesis that high levels of preoperative anxiety or pain catastrophizing are associated with an increased risk of chronic postsurgical pain?"

Anxiety, fear of pain, and pain catastrophizing measure different pain-related constructs, however, these constructs are related close enough to consider them together. ${ }^{1}$ Based on our systematic review and meta-analysis, we found evidence that anxiety and catastrophizing play a role in the development of CPSP. Therefore we recommend that anxiety measures are incorporated in future studies investigating the prediction of CPSP and transition from acute to chronic postoperative pain. We also concluded that validation and proposal of one instrument most suitable for prediction of CPSP was necessary (See chapter 2). Our conclusion was based on 29 included studies in which 14 different instruments were used to assess anxiety or pain catastrophizing. A statistically significant association between anxiety or pain catastrophizing and CPSP was reported by 16 studies (55\%). Furthermore, based on 15 studies providing data enabling meta-analysis, an overall pooled odds ratio was calculated. The risk of CPSP for anxious or catastrophizing patients ranged from 1.55 (95\% confidence interval, 1.10-2.20) to 2.10 (1.49-2.95).

A limitation of the literature study was the presence of heterogeneity, as reflected by the 14 different instruments used for assessment of anxiety and catastrophizing, the 21 outcome measures used, and different statistical analyses applied across the studies included in the review. However, when performing subgroup analyses for the pooled effect sizes, not in all of our analyses significant heterogeneity was present (See chapter 2 , table 5). Moreover, our findings are confirmed by a recent comprehensive literature study and meta-analysis on the association between presurgery emotional distress and postoperative pain outcomes, performed on 47 studies. $^{2}$ Emotional distress was defined 
by catastrophizing, anxiety, fear, depression, or general negative affect/emotional distress. Studies assessing optimism and positive well-being were also included, and analysed using the reversed scores to reflect low well-being. Outcomes included measures of pain intensity, analgesic use, or functional impairment. There were 10 new studies since 2010 in which, similarly to our publication, an assessment was performed of anxiety or catastrophizing as predictor, with a postoperative follow-up of minimally 90 days. Subgroup analyses on all included studies, divided for studies with a follow-up shorter or longer than 2 months revealed that the impact of preoperative distress was most evident on the acute postoperative pain outcomes. However, also for the studies with long-term follow-up modest but significant effect sizes were found.

Because obviously, preoperative fear can play an important role in the perioperative course, it is important to have the disposal of an instrument dedicated to the assessment of the specific characteristics of surgical fear. An instrument developed to this end is the Surgical Fear Questionnaire (SFQ). In chapter 3 and 4 we took a closer look at the SFQ.

The assessment of surgical fear: validation of the Surgical Fear Questionnaire (SFQ)

The SFQ was developed as a specific instrument to assess various aspects of preoperative fear in adult patients. It was intended for use in both clinical and day surgery. Concerning the validation of the SFQ two research questions were formulated:

"Is the surgical fear questionnaire a valid and reliable instrument for the assessment of surgical fear?", and

"What is the sensitivity of the surgical fear questionnaire with regard to the detection of potential differences in surgical fear across time, in the week before cataract surgery, and is there an association between the level of surgical fear assessed by the surgical fear questionnaire, and salivary cortisol and salivary alpha amylase levels?"

To assess whether the SFQ is a valid and reliable instrument for the assessment of surgical fear in adults, data of five previous studies that had applied the SFQ were used. Our results revealed that the SFQ is an eight-item index of surgical fear consisting of two subscales, fear of the short-term (SFQ-s) and fear of the long-term (SFQ-I) aspects of surgery. The SFQ has a high internal consistency, and across all studies we found adequate convergent validity with other instruments assessing state and trait anxiety, catastrophizing, expected pain, and expected recovery. The SFQ also generates insights in the specific aspects of surgical fear which can be directive in targetted preoperative patient information interventions. 
Predictive validity was established using bivariate analyses. The predictive value was not exactly identical for SFQ-S and SFQ-I. In the bivariate analyses described in chapter 3, SFQ-I was more strongly related to pain and global recovery than SFQ-s. This aligns with the findings in the original studies from which the data was derived and which used multivariate analyses, that SFQ-I predicted lower postoperative SF-36 physical functioning and vitality after six or 12 months, ${ }^{3}$ and pain and poor global recovery at six or 12 months follow-up. ${ }^{4,5}$ In contrast, multivariate analyses revealed SFQ-s as a predictor of acute postoperative pain in day surgery patients. ${ }^{6}$ As such, these multivariate results provide additional confirmation for the two factor structure of the scale, because the SFQ-s predicted acute pain, while the SFQ-I predicted long-term outcomes.

Because the five studies included in the analyses for chapter 3 had only applied the SFQ at one point an additional study was performed to examine the sensitivity of the SFQ to detect differences of surgical fear across time, in the week before cataract surgery. We also examined potential predictors of the level and time course of surgical fear. Finally, the association between the level of surgical fear assessed by the SFQ, and state anxiety, expected pain and recovery, and salivary cortisol and salivary alpha amylase levels as biological indices of stress was examined. We found that fear scores assessed at three different time points in the week before surgery were well comparable, except for certain subgroups. The SFQ was sensitive to detect small differences in surgical fear over time before cataract surgery, but these differences were generally of no clinical significance. As for predictors of surgical fear, results indicated that preoperative pain and stress increased fear levels and that several other factors (e.g. education level, antidepressant use) influenced the time course of fear. There was no association between surgical fear and salivary stress hormone levels. An explanation could be that the fear level in the cataract population was too low for the detection of an association with salivary alpha amylase (SAA) and cortisol. Finally, the previous findings with regard to convergent validity with other instruments assessing state anxiety, expected pain, and expected recovery were confirmed.

Several aspects need further consideration in the future. First, we did not find an association of fear with cortisol or SAA. Therefore, the association between salivary stress hormone levels and surgical fear should be studied in patients waiting for surgical interventions that might elicit higher fear levels. Second, the establishment of clinically relevant cut-off levels for the SFQ has not been performed yet. In the studies on prediction of poor postoperative outcome, until now use was made of median split or a continuous scale. However, for the SFQ to be useful to the selection of patients at increased risk of poor postoperative recovery, criteria are needed to select those patients who will benefit most of preoperative fear treatment. 
Predictors of postoperative quality of life after hysterectomy.

In chapter 5 and 6 predictors of quality of life after hysterectomy are assessed in answer to the research question:

"What are the somatic and psychosocial risk and protective factors for pain, depression, physical functioning, global surgical recovery, and well-being after 3 and 12 months, in patients undergoing elective hysterectomy?"

In the next section several types of predictors are further discussed.

\section{Preoperative and acute postoperative pain}

Preoperative pain is the most common predictor of postoperative acute pain across many studies, and preoperative and acute postoperative pain are both the most common predictors of CPSP. ${ }^{7,8}$ In addition, perioperative pain is predictive of many other aspects of postoperative quality of life, as reported by other previous publications (e.g. functional disability, ${ }^{9}$ increased functional limitations, ${ }^{5}$ poor global surgical recovery, ${ }^{3-5}$ physical functioning, ${ }^{3}$ pulmonary complications, ${ }^{10}$ depression ${ }^{11,12}$ ) and also confirmed by the results of this thesis. In our hysterectomy study described in chapter 5 and 6 , we found that preoperative pain was related to CPSP, physical functioning, depression, and well-being, while acute postoperative pain at the PACU or at postoperative day four was predictive of CPSP, physical functioning, global surgical recovery, and the well-being energy subscale. These results emphasize that the prevention of perioperative pain including adequate timing of preventive interventions should have our undivided attention when aiming at better postoperative outcomes.

Because the presence of preoperative pain is a known risk factor of poor postoperative outcome, several predictors referring to preoperative pain were assessed. Among other, the preoperative pain intensity using a numeric rating scale (NRS) and the indication abdominal pain were assessed, and also the indication endometriosis/adenomyo$\operatorname{sis}^{13}$ which is also associated with complaints of pain. Furthermore we separately assessed pain related to the planned hysterectomy and other pain, not related to the planned hysterectomy. Remarkably, the indication abdominal pain did not predict chronic postoperative pain, but was "only" significantly associated with poor physical functioning and, at 3 months only, poor positive well-being. Our prediction models might be confounded by the predictors preoperative hysterectomy-related pain intensity and the indication abdominal pain, since in none of the prediction models they predicted outcome simultaneously. On the other hand, nonhysterectomy-related pain intensity at baseline did significantly predict CPSP together with hysterectomy-related pain intensity. Nonhysterectomy-related pain also predicted higher levels of depression and lower levels of well-being. Importantly, despite the knowledge that preoperative pain is a risk factor for CPSP, when comparing the prevalence of preoperative pain with the prevalence of postoperative pain based on the NRS pain scores, the large reduction 
of pain that we found supports the effectiveness of hysterectomy when (abdominal) pain is the indication for surgery. However, there is room for further reduction of the risk of CPSP after hysterectomy. One of the mechanisms leading to CPSP is hyperalgesia. Interestingly, some studies have shown that pre-existing hyperalgesia abolished after surgical intervention. ${ }^{14,15}$

\section{Psychological factors: fear and pain catastrophizing}

Several psychological factors were revealed as predictor of postoperative quality of life. The role of surgical fear and catastrophizing was already mentioned in chapters 2 to 4. In the hysterectomy study, surgical fear was assessed using the SFQ, similar to previous studies. $^{4-6,16}$ To reduce the number of variables in the multivariate analyses the SFQ was combined with the PCS in an aggregate score, named "surgery-related worries". Because of overlap between the psychological constructs as measured, aggregate scores were considered legitimate, and its use is supported by several other publications. ${ }^{17-19}$ And additional evidence for the coherence between the constructs is provided by a study on pain 3 months after knee and breast surgery: in addition to acute postoperative pain, both state anxiety and pain magnification, as a subscale of pain catastrophizing, predicted CPSP for both types of surgery. One of the conclusions was that "state anxiety and pain magnification seem to constitute psychological risk factors for chronic postsurgical pain relevant in all surgical models." ${ }^{20}$ Considering all six final multivariate prediction models, we found that surgery-related worries predicted CPSP, global surgical recovery, and positive well-being. Although the final prediction models shown in this thesis do not inform about the individual effects of each separate predictor, the information about bivariate effects is available in the supplemental files of chapters 5 and 6 .

Because the validation of the SFQ is an important issue of this thesis, some additional remarks are made with regard to the predictive characteristics of different types of anxiety. In chapter 2 we found that the predictive effects of catastrophizing seemed a little stronger compared to general or more pain-related anxiety. A more recent metaanalysis on the association between presurgery emotional distress and postoperative pain outcomes reported a similar finding. ${ }^{2}$ Pain catastrophizing showed the strongest association with the various outcomes whereas effect sizes for fear were the weakest. We discussed the absence of predictive performance of a specific type of fear, kinesiophobia as measured with the TSK in surgical patients in chapter 2: in none of the four included studies, on musculoskeletal surgery, where the TSK was applied an association was found with CPSP. Including the recent findings by Jackson et al., ${ }^{2}$ it appears that none out of seven studies established an effect for the TSK. Therefore we conclude that there seems no place for the TSK in predictor studies on postsurgical chronic pain. For the SFQ, the existing evidence of predictive value as established, using aggregate scores in chapter 5-6 and as reported in previous studies, ${ }^{3-6}$ was not further expanded by the review of Jackson et al. Specific pain-related or surgical fears are not necessarily the strongest predictors of CPSP. Many of the fear and anxiety concepts, including pain 
catastrophizing show a good deal of overlap and it may be speculated that a general vulnerability profile could underlie the increased risk of CPSP. The significant effects of the aggregate scores used in chapter 5-6 and in previous studies are in support of this.

Psychological factors: depression, optimism, well-being, affect, social support, and abuse In our hysterectomy study we also included other psychological factors that could potentially predict outcome, namely depression, well-being, and optimism. They were combined in an aggregate score named "general psychological robustness" for analyses of the outcomes CPSP, physical functioning, and surgical recovery. In multivariate analyses general psychological robustness appeared to be a predictor of physical functioning only. However, in bivariate analyses we found that depression was associated at $\alpha=0.1$ level positively with outcome CPSP, negatively with physical functioning and, as revealed by a significant interaction term, affected the course of global surgical recovery. Also at $\alpha=0.1$ level in bivariate analyses, well-being energy was negatively associated with CPSP and positively with physical functioning, while positive well-being was positively associated with physical functioning and affected the course of global surgical recovery as revealed by a significant interaction term. Finally, also in bivariate analyses, optimism affected the course of global surgical recovery, as revealed by an interaction term significant at $\alpha=0.1$ level. A protective effect of optimism for postoperative quality of life is reported in several other studies. One study, also using optimism in an aggregate score, reported lower acute pain after breast cancer surgery. ${ }^{17}$ Another study on acute pain after hysterectomy and major joint arthroplasty, reported that optimism was associated with lower acute pain after major joint arthroplasty but not after hysterectomy. ${ }^{21}$ Better long-term recovery was reported in more optimistic patients with regard to pain and global surgical recovery after day surgery, ${ }^{4}$ and with regard to global surgical recovery and general quality of life after clinical surgery. ${ }^{5}$

For the outcomes depression and well-being the aggregate score general psychological robustness was not applied because of the overlap with the respective baseline references. Depression and well-being at follow-up were significantly predicted by their corresponding baseline references. It seems that depression after hysterectomy in many cases is a continuation of a pre-existing state rather than an adverse outcome of hysterectomy and this is in line with the findings of a recent literature review stating "the effect size, the log relative risk of postoperative depressive symptoms increases with higher preoperative depressive symptoms."22 In our study population we found that optimism predicted one of the psychological outcomes, positive well-being. This is in line with previous results, where optimism predicted better mental health and vitality 12 months after surgery. ${ }^{3}$ Negative affect was only assessed for the psychological outcomes depression and well-being and appeared to be negatively associated with wellbeing energy. Finally, more social support predicted increased well-being energy.

We assessed whether the participants had a history of physical or sexual abuse, and found prevalences of $12 \%$ and $19 \%$ respectively. In general, childhood abuse has been 
associated with many types of impaired physical and psychosocial health conditions. ${ }^{23}$ One previous study, performed on hysterectomy patients, evaluated depression, sexual function, and satisfaction with their hysterectomy decision. No differences with regard to sexual functioning or satisfaction were found, however one year after hysterectomy women reporting abuse had significantly higher levels of depression, compared to women not reporting abuse. ${ }^{24}$ In our study population a history of abuse did not predict any of the outcomes assessed. One patient however reported that she suffered from flash-backs of incest, induced by the hysterectomy, resulting in hospitalization at the psychiatric unit of the hospital.

\section{Surgery-related factors}

Several surgery-related predictors were assessed: indication for hysterectomy, type of surgery, type of anesthesia, peroperative blood loss, years of training of the gynecologist, and postoperative self-reported infection. The most striking finding was the association between postoperative self-reported infection and nearly all outcome measures. Infection significantly predicted CPSP, physical functioning, global surgical recovery, depression, and well-being energy. However, as discussed in chapter 5, we were not able to assess whether the infection was still present at the time of evaluation. Neither did we assess details about perioperative preventive measures, nor about the type, severity, or treatment of the infection. But $12.4 \%$ of the patients reporting a surgeryrelated infection at 3 month follow-up, is relatively high when compared to surveillance data on postoperative wound infection in the Netherlands in the period 2012-2014: the reported incidences of postoperative deep or surficial wound infection after abdominal hysterectomy are $1.5 \%(95 \% \mathrm{Cl} 1.0-2.1)$ and after vaginal hysterectomy $0.7 \%(95 \% \mathrm{Cl}$ $0.4-1.4) .{ }^{25}$ The surveillance data are collected using a validated system for reporting and registration of postoperative wound infections, including a strict definition and a followup period of 30 days. In contrast, our self-report assessment, using a less strict definition and a 3 month follow-up period, might at least partly explain the differences in incidence, and do not necessarily have to mean an overestimation since a surgeryrelated infection may present itself other than a wound infection. Apart from infection of the abdominal incision or vaginal cuff, also infected haematoma or abscesses, urinary tract infections, or respiratory infections may occur. ${ }^{26}$ Moreover, previous publications reported infection rates rather similar to ours, of 9 to 13\%, depending on the type of incision, ${ }^{27}$ or even higher. ${ }^{26}$ However, the impact of self-reported infection on multiple postoperative outcomes in our population suggests that, despite educational and organizational intervention programmes such as the Dutch Patient Safety Programme (VMS) theme POWI (prevention of postoperative wound infections) and medical guidelines and interventions such as antibiotic prophylax, ${ }^{28}$ studies focussing at further reduction of surgical site infection after hysterectomy are needed. If these studies lead to improvements in infection prevention, patients may benefit in particular with a decreased risk of CPSP and higher global recovery levels. In order to assess the impact of 
infection in our study population more specifically, we performed post hoc analyses. These (unpublished) analyses revealed that patients who reported a surgery-related infection 3 months after hysterectomy had only a marginally longer stay in the hospital, and reported only marginally higher levels of depression and lower levels of well-being. However, scores on pain intensity, pain interference, global surgical recovery, and physical functioning were significantly worse in women reporting an infection after 3 months compared to those not reporting an infection.

Besides surgery-related infections also some of the indications for hysterectomy appeared to have significant effects on postoperative quality of life, however not across all outcome measures. We found that the indication cervical dysplasia had a negative effect on well-being energy levels, however the effect was less after 12 months compared with 3 months. Patients with the indication endometriosis/adenomyosis and patients with the indication abdominal pain showed relatively large improvements in physical functioning at follow-up. While mild endometriosis can be without symptoms, severe endometriosis is mostly accompanied by pain symptoms, resulting in an indication for surgery. ${ }^{13}$ Closer examination of our data, to assess whether baseline physical functioning levels were lower for patients with indication endometriosis/adenomyosis or indication abdominal pain, and thus leaving more room for improvement, revealed that the mean levels of baseline physical functioning ranged between 76.8 for indication prolapse to 84.7 for indication cervical dysplasia. For endometriosis/adenomyosis the mean level was 81.5 and for indication abdominal pain 79.6, so in the mid-range. However, the minimum scores of both indications were 15 and 20 respectively, among the lowest together with indication prolapse (20) and menorrhagia/metrorrhagia (5), whereas the minimum scores of the other indications were between 25-35. Therefore, in patients with the indications endometriosis/adenomyosis and abdominal pain there might be a larger proportion of patients with lower baseline physical functioning, who might benefit extra of hysterectomy.

Although Pfannenstiel incision is associated with an increased risk of NPP ${ }^{29-31}$ and our study revealed the highest incidence of NPP after 3 months in patients undergoing Pfannenstiel incision, it did not predict CPSP. This might be a result of the fact that median lower abdominal hysterectomy and Pfannenstiel incision were analyzed together to reduce the number of predictors in multivariate analyses. However, in bivariate analyses all five types of incision were assessed, and no effect on CPSP was found. Moreover, in a previously published thesis on chronic pain after hysterectomy of Brandsborg, ${ }^{32}$ no effect of surgery type was found either. A literature review on depression and anxiety after hysterectomy reported postoperative improvements on outcome depression, regardless the type of hysterectomy, ${ }^{22}$ which is in line with our results. Finally, for none of the other physical or psychological outcomes the type of incision was predictive.

With regard to type of anesthesia, general anesthesia was the most prominently applied, in $84 \%$ of all patients. Whether epidural analgesia could be a factor in prevention of CPSP is unclear from our data. There are publications indicating that there is a de- 
creased risk of CPSP after use of epidural analgesia in abdominal surgery, ${ }^{33,34}$ whereas other studies failed to show an effect for chronic pain. ${ }^{35,36}$ However, until now evidence based on extensive systematic reviews is lacking. Brandsborg reported conflicting results on the protective effects of epidural and spinal anesthesia with regard to the occurrence of CPSP after hysterectomy. ${ }^{32}$ We were not able to show a protective effect, which might, at least for the epidural group, partly be the result of small numbers. However, at 3 month follow-up, all 14 patients with combined general and epidural anesthesia were in the no-CPSP group. Importantly, a positive effect on the global surgical recovery score was found for patients receiving spinal anesthesia or a combination of general anesthesia with spinal or epidural anesthesia.

\section{Sociodemographic factors}

Several sociodemographic factors such as age, education level, employment status, and marital status were assessed, in addition to ASA classification as a proxy for general health status. None of these factors were predictive for any of our outcome measures, except ASA classification. We found that with regard to the outcome surgical recovery, patients of ASA class III recovered less well at 12 months compared with patients of ASA class I. As stated in the supplementary file of chapter 5 , to reduce the number of variables in the predictor analyses, ASA was chosen as a proxy for preoperative health status over general health status, body mass index, or the presence of chronic disease as obtained from the comorbidity screening list. ${ }^{37}$ The choice for ASA classification was made because it is readily available and clinically useful.

In summary, we notice that prediction of postoperative quality of life is a matter of various factors belonging to different psychosocial and medical domains. Moreover, different predictors may be associated with different outcomes.

\section{Methodological considerations}

1. The compliance of the participants was good, resulting in relatively low numbers of missing data. When there were missing values on multi-item scales, if $<20 \%$, they were imputed by the participants' mean score on that scale. Single-item variables were not imputed. Although mixed model analyses are robust for missing data, prediction models presenting values for missing data might be difficult to use in practice. As a result, prediction analyses were performed on the basis of total case analyses.

2. Because the large number of predictor and control variables assessed, despite an a priori selection of certain variables, bivariate variable selection was necessary to avoid overload of statistical models.

3. Internal and external validation of our prediction models still needs to be done.

4. All domains of quality of life were covered by validated questionnaires. Pain was measured using the Brief Pain Inventory, physical functioning was measured using the physical functioning subscale of the SF-36, depression using the CES-D en well-being 
using two subscales of the W-BQ 12. Surgical recovery was measured using the global surgical recovery index (GSR), which was developed for validation of other recovery measures and used previously in several other studies on postoperative outcome. This choice provided us with detailed information on the selected outcomes without overlap between questionnaires.

\section{QUALITY OF LIFE AFTER HYSTERECTOMY}

In answer to the final research question:

"What is the prevalence/incidence of chronic pain and depression, and what is the level of physical functioning, global surgical recovery, and well-being after 3 and 12 months, in patients undergoing elective hysterectomy?",

it was concluded in chapter 5 and 6 that recovery after hysterectomy was satisfactory in general. Many aspects regarding the quality of life after hysterectomy have been discussed already. Below, some additional remarks on the outcome CPSP and other outcomes are described.

\section{CPSP}

The importance of pain in the assessment of postoperative quality of life was previously confirmed in the study on the validity of the Postoperative Recovery Profile (PRP) questionnaire: at all postoperative time points abdominal and orthopedic surgery patients ranked pain at place one or two out of 19 items assessing postoperative recovery. ${ }^{38}$ And as mentioned in the introduction, for the hysterectomy study the selection of the outcome measures was based on the framework with core predictors and outcome measures for chronic postsurgical pain in epidemiological studies, in accordance with the article of VanDenKerkhof et al. ${ }^{39}$

A patient was defined as having CPSP if the highest NRS of hysterectomy-related pain in the past week was $\geq 4$, implying moderate or severe pain. Previous studies, using a variety of criteria for CPSP reported prevalences of CPSP after hysterectomy between $5-32 \%,{ }^{40}$ one study even $50 \%,{ }^{16}$ whereas we found a 3 month follow-up prevalence of $10.2 \%$, an incidence of $2.2 \%$, and the 12 month prevalence was $9.1 \%$. Based on the average NRS of hysterectomy-related pain in the past week the prevalence at 3 and 12 months was $8.0 \%$ and $7.0 \%$, respectively. Compared with the baseline prevalence of $50 \%$ hysterectomy-related pain, hysterectomy can be considered successful in reducing pain. But the prevalence of $10.2 \%$ and $9.1 \%$ of CPSP suggests there is room for further improvements. The results of the predictor analyses give direction to the most important factors to aim at. In addition, detailed descriptions of the baseline characteristics for patients with and without CPSP, are presented in the supplementary files of 
chapter 5. Interestingly, they show that the CPSP patients fare less well at baseline in many aspects, albeit not always significant, when compared to patients without CPSP.

The seven self-report items of the DN4, named DN4-interview, were assessed. This gave us the opportunity to pay extra attention to the role of the neuropathic component of CPSP. The prevalence of hysterectomy-related neuropathic pain increased from $10 \%$ at baseline to $24 \%$ at postoperative day 4 , and subsequently decreased to $5 \%$ and $4 \%$ at 3 and 12 months respectively. NPP at 3 months was reported most after Pfannenstiel incision (14.0\%) and with general anesthesia (5.7\%). The incidence of NPP, i.e. the number of patients reporting NPP at follow-up who did not report NPP at baseline, was $3.8 \%$ at three months (14 of 372, missing data 56) and 2.4\% at 12 months (unpublished, eight of 338, missing data 90). A previous study of Clarke-Pearson and Geller, on complications after hysterectomy, stated that the rate of neuropathy after benign hysterectomy tends to be towards $0.2 \% .{ }^{27}$ Another study among 866 women on the risk of NPP after Pfannenstiel incision for cesarean delivery or abdominal hysterectomy found an incidence of NPP (entrapment of N. iliohypogastric or N. ilioinguinal) of $2.5 \%$, based on patient interview combined with physical examination. ${ }^{30}$ After laparoscopic gynecological procedures an incidence of $4.9 \%$ was found for nerve entrapment, that is, when fascial closure was involved. ${ }^{41}$ Thus, our study shows incidences of NPP a similar range as has been reported in the latter two studies. However, these incidences are much higher as reported by Clarke-Pearson and Geller. Reasons for these variations might be the differences in assessment of NPP based on use of a self-report instrument versus additional testing by a clinician, or prospective versus retrospective data collection. Interestingly, we found that in group CPSP 53\% indicated NPP at day four and $46 \%$ at three months, while in group no CPSP NPP reduced from $19 \%$ at day four to $1 \%$ after three months. With regard to the 12 month results a similar pattern was found (unpublished). Time to recovery depends on the type of nerve injury: for neurapraxia, a mild injury caused by external compression to the nerve, recovery is within weeks or months. In case of axonotmesis, where the axon is damaged by profound compression or traction, without harm of the Schwann cells, recovery will take much longer. When the nerve is completely interrupted, called neurotmesis, recovery might be impossible without nerve surgery. ${ }^{42}$ As discussed in chapter 5 , unfortunately our data did not allow establishing whether nerve injury in the group no CPSP was less severe as compared to group CPSP. One study reporting an incidence of $1.8 \%$ of lower extremity NPP after gynecologic surgery concluded that most cases resolve over time. ${ }^{43}$ As already explained in the introduction, not all CPSP results from NPP and our data show that nearly two third of the CPSP at 12 months seems not to be neuropathic. Therefore interventions aiming at the reduction of CPSP should be based on a broad view upon possible underlying mechanisms.

We used intensity of hysterectomy-related pain for the outcome CPSP although many other characteristics of pain were also assessed, such as onset, intermittence, location, interference, analgesic use, and other pain treatment, partly derived from the 
Brief Pain Inventory Short Form (BPI-SF). A composite score might be a more comprehensive option for assessment of CPSP, but there is no consensus yet about what measure to use. Moreover, concerning the currently widely used definition of CPSP of Macrae, ${ }^{44}$ suggestions are published for further refinement with regard to the criteria onset, duration, continuity, including also a new criterion concerning the location of pain. $^{45}$

To further validate the measure used for CPSP after hysterectomy, several other outcomes for the CPSP group and the no-CPSP group were compared. This revealed that the definition as used reflected indeed (statistically) significant impact of CPSP: "For the outcomes (mean) pain intensity, pain interference, analgesia use, SF-36 physical activity, GSR, and proportion of woman able to perform daily activities again, the scores of patients in the CPSP group are significantly less favorable than for patients in the no-CPSP group, at both 3 and 12 months" (chapter 5). Even mean and highest nonhysterectomy-related pain intensity scores were significantly higher in the CPSP group.

Another option for a more comprehensive assessment is the use of pain interference instead of the pain intensity score, because it assesses pain in relation to several aspects of normal daily living. In our study pain interference was assessed, as part of the validated BPI. Pain interference was not used as an outcome in multivariate analyses because it was assessed for all pain complaints, while our primary aim was to assess predictors for hysterectomy-related pain. To obtain a clearer view on the association between pain (NRS highest hysterectomy-related pain 0-10) at follow-up, the other outcomes, and also pain interference, correlations between the 12 month outcomes are presented in the table below. When comparing the correlations of pain versus the correlations of pain interference with the other outcomes, the correlations of pain with the other outcomes are clearly lower compared with the correlations of pain interference. This illustrates that the pain scores reflect an issue more on itself when compared with pain interference, as may be expected. Therefore, the choice of which outcome to use may also depend on the purpose it is assessed for. And as long as there is no consensus, assessment of multiple existing pain-related outcomes may be the best solution. 
Table. Pearson correlations between recovery outcomes 12 months after hysterectomy.

\begin{tabular}{|c|c|c|c|c|c|c|c|c|}
\hline & & 1 & 2 & 3 & 4 & 5 & 6 & 7 \\
\hline 1 & Pain & 1 & & & & & & \\
\hline 2 & Physical functioning & $-0.283^{* *}$ & 1 & & & & & \\
\hline 3 & Surgical recovery & $-0.497 * *$ & $0.420 * *$ & 1 & & & & \\
\hline 4 & Depression & $0.213^{* *}$ & $-0.391 * *$ & $-0.419 * *$ & 1 & & & \\
\hline 5 & Well-being energy & $-0.213 * *$ & $0.469 * *$ & $0.408^{* *}$ & $-0.755^{* *}$ & 1 & & \\
\hline 6 & Positive well-being & $-0.131^{*}$ & $0.321^{* *}$ & $0.300 * *$ & $-0.778^{* *}$ & $0.721^{* *}$ & 1 & \\
\hline 7 & Pain interference & $0.352 * *$ & $-0.658 * *$ & $-0.396 * *$ & $0.479 * *$ & $-0.527 * *$ & $-0.403 * *$ & 1 \\
\hline
\end{tabular}

Pain: NRS highest hysterectomy-related pain last week. Physical functioning: SF-36 physical functioning subscale. Surgical recovery: Global surgery recovery scale (GSR). Depression: CES-D. Well-Being: Well-Being Questionnaire 12, energy and positive well-being subscales. Pain interference: BPI pain interference, for hysterectomy- and nonhysterectomy-related pain. *Significant at $p=0.05$ level, ${ }^{* *}$ significant at $p=0.01$ level.

\section{Other outcomes}

Concerning the outcome pain we found a firm decrease from the pre- to postoperative period, with a relatively low prevalence of CPSP. However, besides pain other outcomes are important to reflect the quality of life after hysterectomy. With regard to the outcome physical functioning, in our study pre-to-postoperative changes were small and less prominent than the changes in pain. Furthermore, the level of global surgical recovery was high in general. And as already concluded in a recent literature review and meta-analysis of Darwish et al., hysterectomy generally does not lead to depression. Based on pooled data of 10 studies published between 1982 and 2011, preoperative depression was reported in 1000 out of 3367 patients (29.7\%), postoperative depression was reported in 489 out of 3133 patients (15.6\%). They concluded that "data from before and after studies suggest that hysterectomy for benign gynecological conditions ... may be positively rather than adversely associated with depression". ${ }^{22}$ Our own data presented in chapter 6 once more confirm these findings, albeit to a certain extent: a small decrease from pre-to-postoperative prevalence of depression was shown, with percentages of $24 \%, 19 \%$, and $21 \%$ at baseline, 3 and 12 months respectively. Postoperative levels of well-being increased slightly when compared with baseline levels, albeit that the increase for positive well-being at 12 months was not significant. Finally, some previous studies have reported that hysterectomy might affect feelings of femininity. We found that in the majority of woman feelings of femininity were unaffected after hysterectomy. 


\section{FUTURE IMPLICATIONS}

\section{Practical implications}

Perioperative optimization: let's not wait for more evidence

In times of increasing costs of health care and subsequent cutbacks in health care expenditures, preventive health care measures might not always be the most easy to implement. However, the logical step after establishing risk factors of poor postoperative quality of life, is the implementation of preventive measures. The results of this thesis do suggest that by optimizing the patients' preoperative status, patients may benefit from improved postoperative quality of life. And for mixed types of surgery we show that preoperative anxiety and catastrophizing is associated with an increased risk of CPSP, which also suggests that preoperative treatment of anxiety/catastrophizing might reduce the risk of CPSP. Moreover, from other studies on different surgery types we know that besides anxiety and catastrophizing, also other risk factors, similar to those we established in our hysterectomy population, play a role. ${ }^{4,5}$ Therefore, based on the evidence for the existence of various risk factors, more attention should be paid to the optimization of patients' perioperative psychosocial and physical condition.

A critical point however, might be the limited evidence for perioperative interventions targetting established risk factors, as already mentioned in the introduction where prehabilitation programmes were discussed. And even when interventions might show immediate success, will they also improve long-term outcomes of surgery? Similarly, in chapter 2, based on the data of the literature review our conclusion was that it was too early for widespread implementation of preoperative screening of anxiety as a predictor of CPSP. However, notwithstanding the possible shortcomings mentioned above, because of the potential high impact for patients at risk, postponing perioperative interventions until more evidence on efficacy has been gained, may carry the risk of suboptimal treatment of many patients who have their surgery now. Therefore, when combining the evidence for established predictors of surgical outcome in addition to the upcoming evidence for prehabilitation programmes, and the existing knowledge on psychological and perioperative medical treatment options, this should be enough to focus on a perioperative optimization programme targeting both psychosocial, physical, and medical aspects. Several suggestions, using the existing literature, are described below.

\section{Psychosocial assessment}

Patients with preoperative psychological vulnerability, irrespective of whether it is based on fear of surgery, catastrophizing, depression, low optimism, or low well-being, are at increased risk of poor outcome after surgery. Moreover, from chapter 4 we learn that from the patient's perspective, preoperative fear treatment would be appreciated in some cases, even before low risk cataract surgery. Therefore it would be preferable 
to add a psychological assessment to the preoperative screening. Since many years it is common sense for patients visiting Dutch pain clinics to complete a psychological assessment at their first visit. Similarly, surgery patients for elective procedures deserve a comprehensive/holistic preoperative intake, not limited to physical aspects alone. From chapter 3 and 4 we know that preoperative assessment using the Surgical Fear Questionnaire fear renders clues for additional preoperative information supply or fear treatment, and can serve as a predictor of outcome. In addition the general psychological measures depression and optimism should be included. Furthermore pain catastrophizing, as a powerful predictor, several outcome expectancies and well-being are worth considering. Electronical assessment, preferably completed at home in advance, would provide a psychological profile, allowing for targeted preoperative interventions, without logistic burden at the preoperative assessment clinic. We propose to implement this at least for major surgery, until further evidence on the selection of measurement instruments and interventions has been obtained. Alternatively one comprehensive instrument could be used with short questions instead of all full scales as mentioned above. This could yield a risk profile similar to the yellow flags as used for low back pain patients. ${ }^{46}$

\section{Psychosocial interventions}

Currently, several psychological treatment options are available for preoperative psychosocial optimization. In the section below, some of the existing evidence on psychological treatment options is presented. For some of the interventions many studies are performed, summarized in literature reviews, for other interventions less evidence is available. Moreover, for some of the interventions effect sizes may be relatively large, whereas for other the established effect sizes are small, results are inconsistent, or the quality of evidence is weak. Therefore, further studies on the efficacy of optimal psychological interventions are needed. However, meanwhile brief preoperative, or if indicated directly postoperative, psychological interventions, directed at diminishing anxiety and negative cognitions relating to surgery and its outcomes, should be applied according to the current state of knowledge. ${ }^{47}$ One of the options is to provide more (tailored) patient education. Education can consist of providing information only, or can include interventions such as counseling and behavioural therapy. ${ }^{48}$ Depending on the type of education it can be provided by the surgeon, the anesthesiologist, a nurse, or a psychologist. Several studies provide results on patient information concerning the surgical procedure or what a patient may feel, ${ }^{49}$ or different types of skills training such as guided imagery or training for pain coping. ${ }^{49-55}$ For the treatment of preoperative pain catastrophizing, with the aim of reducing acute and long-term postoperative pain, cognitive behavioural therapy (CBT) was studied in a meta-analysis and revealed very promising results: the calculations suggest a relevant reduction of CPSP when high catastrophizers would receive cognitive behavioural therapy. ${ }^{56}$ Several publications indicate that baseline expectations can play an important role in postoperative recov- 
ery. ${ }^{57,58}$ A recent study published results concerning a short intervention aiming at optimizing preoperative expectations in cardiac surgery patients. In the intervention group patient expectations were successfully ameliorated. ${ }^{59}$ However, their effect on recovery-related outcomes remains to be determined. In the context of this thesis, the knowledge that expectations can be modified is relevant, however one other literature review draws a more prudent conclusion with regard to the role of patients expectations. ${ }^{60}$ Furthermore, evidence exists for the application of hypnosis, ${ }^{49,54,61}$ and relaxation or music listening. ${ }^{49,54,55,62}$ Finally, another option for treatment of preoperative anxiety is pharmacological treatment. ${ }^{63}$ However, also the effect of pharmacological treatment on recovery-related outcomes remains to be determined.

Apart from the current state of evidence, the selection of treatment may depend on the time available before surgery, cost-effectiveness, and patient preference. It is important that the intervention of choice matches with the personality of the patient: there is evidence that locus of control and self-efficacy moderate the effects of psychological interventions. ${ }^{64}$ As a result, in the worst case, patients might experience treatment preparation as threatening instead of helpful. Thus, awaiting additional evidence for several interventions, selection and implementation of available treatment options should be performed with great caution on carefully selected patient groups.

\section{Physical assessment}

The current preoperative screening is focussed at physical parameters. Blood pressure, blood glucosis level, anemia, and coagulation status are routinely assessed, and if necessary optimized before surgery because disruption implies immediately an increased risk of per- and postoperative complications. When cardiothoracic surgery came up also extra attention to respiratory function was paid, and more recently malnutrition is addressed. For orthopedic surgery interest for preoperative physical function like walking distance is obvious. The predictor physical functioning in our hysterectomy population was only assessed in multivariate analyses for the outcome physical functioning because of our a priori selection. However, in bivariate analyses physical functioning was associated with the outcomes CPSP and depression, and ASA classification, as a proxy for physical state, was associated with global surgical recovery. Therefore it would be useful to include a measure of physical functioning in preoperative screening.

\section{Physical optimization}

After assessment of preoperative physical functioning preoperative optimization of physical parameters should be implemented at a larger scale. It is based on the principle that a physically healthy person is better able to cope with physiological stress, as caused by the surgery. ${ }^{65}$ Prehabilitation in terms of respiratory optimization before cardiac surgery ${ }^{66}$ or physical exercise before orthopedic surgery has been implemented already, ${ }^{67,68}$ however should be applied more widespread across patients at risk for all 
types of surgery. Group sessions can be implemented at a larger scale and regular basis for cost effective preoperative physical exercise programmes.

\section{Pain}

Despite many alarming publications that perioperative pain causes a lot of personal and societal burden, despite many intervention studies on mainly acute postoperative pain, and despite initiatives aiming at improving postoperative pain management such as the Prospect initiative (procedure specific postoperative pain management), the issue of acute postoperative pain seems hard to tackle. Concerning pharmaceutical treatment, increasing evidence appears in favor of the preemptive use of gabapentin or pregabalin for the prevention of acute postoperative pain. Recent review articles and metaanalyses reveal positive results in reduction of acute postoperative pain in mixed surgery $^{69}$ and also in abdominal hysterectomy ${ }^{70}$ populations. Studies on the impact of gabapentinoids on the reduction of CPSP are still limited in number, but show promising results. ${ }^{69,71-73}$ However, one literature review on systemic drugs for the prevention of

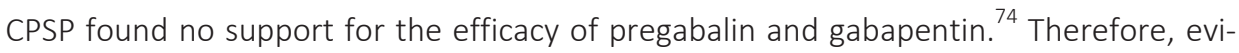
dence for the perioperative use of pregabalin or gabapentin with the aim of prevention of CPSP is contradictory and needs further study. Also ketamine has been suggested for the prevention of chronic pain: Chaparro et al. reported a modest effect of ketamine, compared with placebo, ${ }^{74}$ which is in line with the conclusion of a recent publication on novel strategies for the prevention of CPSP. ${ }^{47}$ Therefore, perioperative use of pregabalin, gabapentin might be successful at least in the reduction of acute postoperative pain, while ketamine might prevent CPSP after hysterectomy and other types of surgery. Finally other anesthesiological treatment options such as epidural analgesia, trunk blocks, or patient-controlled analgesia might need reconsideration.

The findings of our hysterectomy study should reinforce the discussion on surgical techniques among gynecologists, considering further prevention and treatment of NPP with the aim of reduction of CPSP. Many suggestions for improvement with regard to surgical interventions are proposed in the publications referred to before. ${ }^{27,30,41-43}$

Acute pain service (APS) teams are still facing a great challenge in the treatment of postoperative pain and need maximum support. We promote extra attention by nurses for the signals of NPP, starting from the day of surgery. Not only pain intensity, but also pain characteristics should be assessed and indications of NPP should trigger adequate interventions (further assessment of potential nerve lesion by a pain specialist, adaptation of pain medication). An interesting initiative might be the establishment of Transitional Pain Service (TPS) teams as described by Clarke et al. ${ }^{47}$ TPS teams do not only focus on the preoperative selection of patients at increased risk of acute or chronic postsurgical pain, but also focus at detection of patients at risk of persistent postoperative opioid use. Patients at risk receive a teaching programme by a nurse practitioner and contact with primary care is established while still in the hospital. After discharge a TPS visit is scheduled for follow-up, while phone calls are done by the TPS coordinator 
during the period until the follow-up visit. These patients receive a combination of psychological treatment, complementary alternative treatments such as acupuncture, and traditional pharmacological treatment. In fact, the establishment of a TPS could be created by expanding the time span of the existing APS teams, from only postoperative, to pre- and postoperative including postdischarge. In addition, recently agreement was expressed about the necessity of a multidisciplinary approach by pain centers and pain clinics in the treatment of chronic pain patients. ${ }^{75}$ Similarly, in the transformation process from APS to TPS an expansion into a more multidisciplinary approach is worth considering. As such the implementation of a TPS would perfectly match with the concept of a more comprehensive preoperative assessment and prehabilitation programme.

\section{Postoperative infection}

Although not studied in detail, our results indicate that additional efforts aiming at further reduction of postoperative infections after hysterectomy might positively affect many aspects of postoperative quality of life. By paying more attention to postoperative infections during preoperative counseling, patients might be able to react at an earlier stage to signs of infection. Furthermore, since length of stay after hysterectomy has been reduced to one or two days postoperative monitoring after discharge of signs of infection, but also of pain and other aspects of recovery, can be optimized using Ehealth technology. ${ }^{76}$

\section{Assessing postoperative quality of life and recovery}

In our introduction we mentioned the very compact, one-item global surgical recovery index $(G S R)^{77}$ which we used in our hysterectomy study. For the GSR, until now, no established cut-off for good versus poor recovery is available. In a previous study poor global recovery was defined as a GSR score $\leq 80 \%{ }^{5}$ Explorative subgroup analyses of our hysterectomy data revealed that at 3 months follow-up the interquartile range (IQR) of the GSR in group CPSP was 50-80, and 80-100 in group no CPSP. We propose to use a cut-off of $80 \%$ as a rule of thumb, until further validity data are available.

To evaluate a patients postoperative quality of life at a glance, instead of a numerical score a radar plot illustrating the most relevant outcomes might be helpful. In the figure below an example is given including the most relevant outcome measures as used in the hysterectomy study. For the example all outcomes are rescaled to 0-100. Notice that the figure changes from a caterpillar-like pattern in case of poor quality of life towards a butterfly in case of adequate quality of life. In addition colors can be used to indicate poor (red), moderate (yellow, not shown in the plot below), and adequate quality of life (green), similar to the signalling function of red and yellow flags, as used for the presence of prognostic factors in low back pain. ${ }^{46,78}$ However, for each type of surgery an additional surgery-specific outcome measure should be presented too, e.g. bowel movement, range of motion, improved hear loss. 


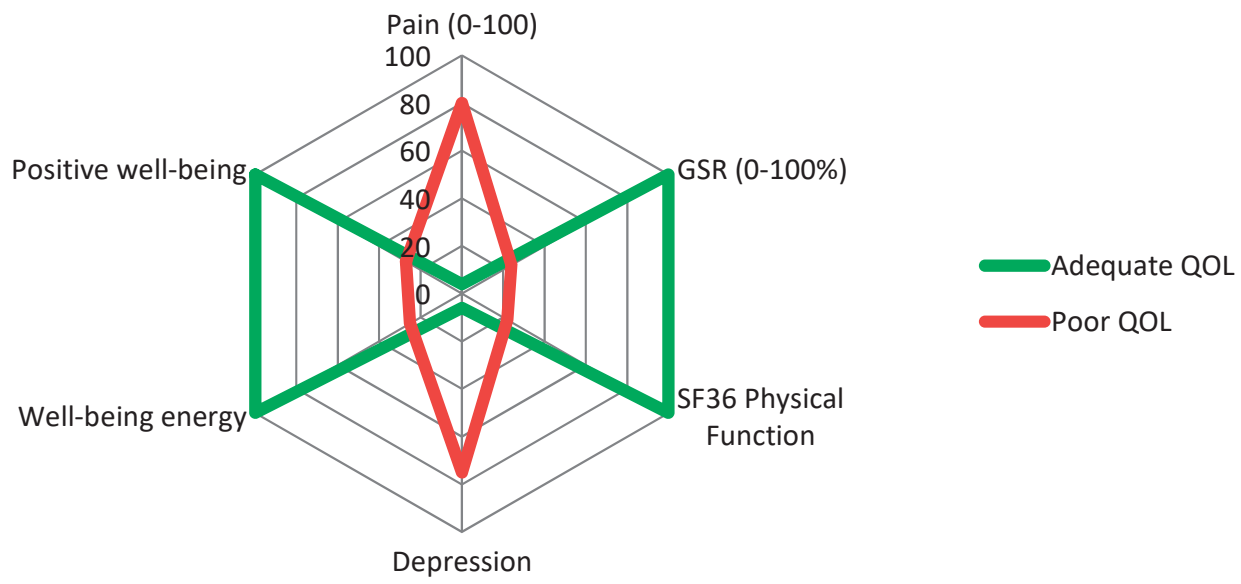

Figure. Radar plot postoperative quality of life

Implications for future research

Based on the heterogeneity as established in chapter 2, there is need for large prospective studies allowing powerful multivariate analyses to establish whether some anxiety related constructs are more important than others, and which assessment instrument or set of instruments is the most suitable for predicting CPSP. Results should be clearly presented, using uniform outcome measures that make statistical pooling possible. The establishment of one or a strictly limited number of "standard" anxiety measures and other predictors included in every study would facilitate future comparison of results.

There is need for validation of existing prediction models on physical and psychological recovery. Validated prediction models reveal insight in postoperative recovery, which can be helpful for clinicians in refining the established indications for surgery, and for patients in their decision about surgery.

(Epi-)genetic factors may play an important role in the prediction of CPSP. In the cohort study on hysterectomy patients (epi-)genetic data were collected and analyses are in preparation. Future results should be incorporated in predictor analyses.

Further studies are needed to assess which interventions targeted toward reducing preoperative anxiety might be worth implementing in the prevention of CPSP. Also other interventions for preoperative tune-up need scientific evidence for their costeffectiveness: the association between optimizing baseline conditions and improved postoperative outcome must become clear. The selection of patients who will profit most of preventive interventions, when and how interventions to apply, are important research questions.

With regard to the outcome pain, until now there is only a limited number of studies on preemptive pain treatment starting earlier than $24 \mathrm{~h}$ preoperative. Further research is needed to is assess whether reduction of existing preoperative pain by prolonged, 


\section{Chapter 7}

pro-active, preoperative pain treatment can reduce acute postoperative and, subsequently, chronic pain.

With regard to the validity of the Surgical Fear Questionnaire, further studies need to establish cut-off levels to facilitate discrimination between high and low fear patients.

For better comparison of results more uniformity across studies is needed. Consensus meetings should be performed about what instruments should be used for assessment of both general and surgery-specific postoperative recovery. After establishment of the main outcomes, for the visualization of the recovery level an app could be developed which creates a radar plot of the main results.

\section{CONCLUSION}

To improve postoperative quality of life, a multidisciplinary approach is mandatory. The current state of knowledge already offers many tools ready for use. These tools concern knowledge on risk factors for impaired recovery, knowledge on instruments needed for a holistic baseline assessment of the surgical patient, including psychosocial measures, physical functioning, and pain, and knowledge on pre-, per-, and postoperative interventions necessary for improved outcome. The challenge is to make the step forward now and carefully implement these tools based on the current evidence, notwithstanding the limited evidence in several domains. However, this thesis started with: "Best care always..." Well, "always" implies also: now. The todays patient might be seriously undertreated if we would wait until the evidence of future studies has been obtained. 


\section{REFERENCES}

1. Vancleef LM, Vlaeyen JW, Peters ML Dimensional and componential structure of a hierarchical organization of pain-related anxiety constructs. Psychol Assess. 2009; 21(3): 340-351.

2. Jackson T, Tian P, Wang Y, et al. Toward Identifying Moderators of Associations Between Presurgery Emotional Distress and Postoperative Pain Outcomes: A Meta-Analysis of Longitudinal Studies. J Pain. 2016.

3. Peters ML, Sommer M, van Kleef $M$, et al. Predictors of physical and emotional recovery 6 and 12 months after surgery. Br J Surg. 2010; 97(10): 1518-1527.

4. Hoofwijk DM, Fiddelers AA, Peters ML, et al. Prevalence and Predictive Factors of Chronic Postsurgical Pain and Poor Global Recovery 1 Year After Outpatient Surgery. Clin J Pain. 2015; 31(12): 1017-1025.

5. Peters ML, Sommer M, de Rijke JM, et al. Somatic and psychologic predictors of long-term unfavorable outcome after surgical intervention. Ann Surg. 2007; 245(3): 487-494.

6. Gramke HF, de Rijke JM, van Kleef M, et al. Predictive factors of postoperative pain after day-case surgery. Clin J Pain. 2009; 25(6): 455-460.

7. Katz J, Seltzer Z Transition from acute to chronic postsurgical pain: risk factors and protective factors. Expert Rev Neurother. 2009; 9(5): 723-744.

8. Kehlet H, Jensen TS, Woolf CJ Persistent postsurgical pain: risk factors and prevention. Lancet. 2006; 367(9522): 1618-1625.

9. Abbott AD, Tyni-Lenne R, Hedlund R Leg pain and psychological variables predict outcome 2-3 years after lumbar fusion surgery. Eur Spine J. 2011; 20(10): 1626-1634.

10. Tzani P, Chetta A, Olivieri D Patient assessment and prevention of pulmonary side-effects in surgery. Curr Opin Anaesthesiol. 2011; 24(1): 2-7.

11. Ghoneim MM, O'Hara MW Depression and postoperative complications: an overview. BMC Surg. 2016; 16(1): 5.

12. Goebel S, Steinert A, Vierheilig C, et al. Correlation between depressive symptoms and perioperative pain: a prospective cohort study of patients undergoing orthopedic surgeries. Clin J Pain. 2013; 29(5): 392-399.

13. Giudice LC, Kao LC Endometriosis. Lancet. 2004; 364(9447): 1789-1799.

14. He W, Liu X, Zhang Y, et al. Generalized hyperalgesia in women with endometriosis and its resolution following a successful surgery. Reprod Sci. 2010; 17(12): 1099-1111.

15. Kosek E, Ordeberg G Abnormalities of somatosensory perception in patients with painful osteoarthritis normalize following successful treatment. Eur J Pain. 2000; 4(3): 229-238.

16. Pinto PR, Mclntyre T, Nogueira-Silva C, et al. Risk factors for persistent postsurgical pain in women undergoing hysterectomy due to benign causes: a prospective predictive study. J Pain. 2012; 13(11): 1045-1057.

17. Bruce J, Thornton AJ, Scott NW, et al. Chronic preoperative pain and psychological robustness predict acute postoperative pain outcomes after surgery for breast cancer. Br J Cancer. 2012; 107(6): 937-946.

18. Campbell P, Bishop A, Dunn KM, et al. Conceptual overlap of psychological constructs in low back pain. Pain. 2013; 154(9): 1783-1791.

19. de Rooij A, Steultjens MP, Siemonsma PC, et al. Overlap of cognitive concepts in chronic widespread pain: an exploratory study. BMC Musculoskelet Disord. 2011; 12: 218.

20. Masselin-Dubois A, Attal N, Fletcher D, et al. Are psychological predictors of chronic postsurgical pain dependent on the surgical model? A comparison of total knee arthroplasty and breast surgery for cancer. J Pain. 2013; 14(8): 854-864.

21. Pinto PR, Mclntyre T, Araujo-Soares V, et al. Differential predictors of acute post-surgical pain intensity after abdominal hysterectomy and major joint arthroplasty. Ann Behav Med. 2015; 49(3): 384-397.

22. Darwish M, Atlantis E, Mohamed-Taysir T Psychological outcomes after hysterectomy for benign conditions: a systematic review and meta-analysis. Eur J Obstet Gynecol Reprod Biol. 2014; 174: 5-19. 
23. Thombs BD, Bernstein DP, Ziegelstein RC, et al. A brief two-item screener for detecting a history of physical or sexual abuse in childhood. Gen Hosp Psychiatry. 2007; 29(1): 8-13.

24. Wukasch RN The impact of a history of rape and incest on the posthysterectomy experience. Health Care Women Int. 1996; 17(1): 47-55.

25. PREZIES, Referentiecijfers 2012-2014: Postoperatieve Wondinfecties. 2016, Rijksinstituut voor Volksgezondheid en Milieu. Ministerie van Volksgezondheid en Sport: the Hague, the Netherlands. p. 1-49.

26. Hodges KR, Davis BR, Swaim LS Prevention and management of hysterectomy complications. Clin Obstet Gynecol. 2014; 57(1): 43-57.

27. Clarke-Pearson DL, Geller EJ Complications of hysterectomy. Obstet Gynecol. 2013; 121(3): 654-673.

28. Van Eyk N, van Schalkwyk J Antibiotic prophylaxis in gynaecologic procedures. J Obstet Gynaecol Can. 2012; 34(4): 382-391.

29. Huikeshoven FJ, Dukel L The bikini incision: nice, but not without painful complications. Ned Tijdschr Geneeskd. 1998; 142(26): 1481-1483.

30. Loos MJ, Scheltinga MR, Mulders LG, et al. The Pfannenstiel incision as a source of chronic pain. Obstet Gynecol. 2008; 111(4): 839-846.

31. Luijendijk RW, Jeekel J, Storm RK, et al. The low transverse Pfannenstiel incision and the prevalence of incisional hernia and nerve entrapment. Ann Surg. 1997; 225(4): 365-369.

32. Brandsborg B, Pain following hysterectomy: Epidemiological and clinical aspects, in Danish Pain Research Center, Aarhus University, Noerrebrogade 44, 8000-Aarhus, Denmark. 2010, Aarhus University Denmark.

33. Bouman EA, Theunissen M, Bons SA, et al. Reduced Incidence of Chronic Postsurgical Pain after Epidural Analgesia for Abdominal Surgery. Pain Pract. 2013.

34. Lavand'homme $\mathrm{P}$, De Kock $\mathrm{M}$, Waterloos $\mathrm{H}$ Intraoperative epidural analgesia combined with ketamine provides effective preventive analgesia in patients undergoing major digestive surgery. Anesthesiology. 2005; 103(4): 813-820.

35. Haythornthwaite JA, Raja SN, Fisher B, et al. Pain and quality of life following radical retropubic prostatectomy. J Urol. 1998; 160(5): 1761-1764

36. Katz J, Cohen L Preventive analgesia is associated with reduced pain disability 3 weeks but not 6 months after major gynecologic surgery by laparotomy. Anesthesiology. 2004; 101(1): 169-174.

37. Berg van den J, Wulp van der C, Rapport van de Werkgroep Revisie POLS-Gezondheidsenquête 1999. 2003, Statistics Netherlands (CBS): Heerlen.

38. Allvin R, Svensson E, Rawal N, et al. The Postoperative Recovery Profile (PRP) - a multidimensional questionnaire for evaluation of recovery profiles. J Eval Clin Pract. 2011; 17(2): 236-243.

39. VanDenKerkhof EG, Peters ML, Bruce J Chronic pain after surgery: time for standardization? A framework to establish core risk factor and outcome domains for epidemiological studies. Clin J Pain. 2013; 29(1): 2-8.

40. Brandsborg B, Nikolajsen L, Kehlet $\mathrm{H}$, et al. Chronic pain after hysterectomy. Acta Anaesthesiol Scand. 2008; 52(3): 327-331.

41. Shin JH, Howard FM Abdominal wall nerve injury during laparoscopic gynecologic surgery: incidence, risk factors, and treatment outcomes. J Minim Invasive Gynecol. 2012; 19(4): 448-453.

42. Bradshaw AD, Advincula AP Postoperative neuropathy in gynecologic surgery. Obstet Gynecol Clin North Am. 2010; 37(3): 451-459.

43. Bohrer JC, Walters MD, Park A, et al. Pelvic nerve injury following gynecologic surgery: a prospective cohort study. Am J Obstet Gynecol. 2009; 201(5): 531 e531-537.

44. Macrae WA Chronic pain after surgery. Br J Anaesth. 2001; 87(1): 88-98.

45. Werner MU, Kongsgaard UE I. Defining persistent post-surgical pain: is an update required? Br J Anaesth. 2014; 113(1): 1-4.

46. Nicholas MK, Linton SJ, Watson PJ, et al. Early identification and management of psychological risk factors ("yellow flags") in patients with low back pain: a reappraisal. Phys Ther. 2011; 91(5): 737-753.

47. Clarke H, Poon M, Weinrib A, et al. Preventive analgesia and novel strategies for the prevention of chronic post-surgical pain. Drugs. 2015; 75(4): 339-351. 
48. Pellise F, Sell P, EuroSpine Patient Line Task Force Patient information and education with modern media: the Spine Society of Europe Patient Line. Eur Spine J. 2009; 18 Suppl 3: 395-401.

49. Powell R, Scott NW, Manyande A, et al. Psychological preparation and postoperative outcomes for adults undergoing surgery under general anaesthesia. Cochrane Database Syst Rev. 2016(5): CD008646.

50. Doering S, Katzlberger F, Rumpold G, et al. Videotape preparation of patients before hip replacement surgery reduces stress. Psychosom Med. 2000; 62(3): 365-373.

51. Guo P Preoperative education interventions to reduce anxiety and improve recovery among cardiac surgery patients: a review of randomised controlled trials. J Clin Nurs. 2015; 24(1-2): 34-46.

52. Gurusamy KS, Vaughan J, Davidson BR Formal education of patients about to undergo laparoscopic cholecystectomy. Cochrane Database Syst Rev. 2014(2): CD009933.

53. Johansson K, Nuutila L, Virtanen $\mathrm{H}$, et al. Preoperative education for orthopaedic patients: systematic review. J Adv Nurs. 2005; 50(2): 212-223.

54. Nelson EA, Dowsey MM, Knowles SR, et al. Systematic review of the efficacy of pre-surgical mind-body based therapies on post-operative outcome measures. Complement Ther Med. 2013; 21(6): 697-711.

55. Scheel J, Parthum A, Dimova V, et al. [Psychological prophylaxis training for coping with postoperative pain. Long-term effects]. Schmerz. 2014; 28(5): 513-519.

56. Burns J, Moric M Psychosocial factors appear to predict postoperative pain: Interesting, but how can such information be used to reduce risk? Tech Reg Anesth Pain Manag. 2011; 15(3): 90-99.

57. Auer CJ, Glombiewski JA, Doering BK, et al. Patients' Expectations Predict Surgery Outcomes: A MetaAnalysis. Int J Behav Med. 2016; 23(1): 49-62.

58. Mondloch MV, Cole DC, Frank JW Does how you do depend on how you think you'll do? A systematic review of the evidence for a relation between patients' recovery expectations and health outcomes. CMAJ. 2001; 165(2): 174-179.

59. Laferton JA, Auer CJ, Shedden-Mora MC, et al. Optimizing preoperative expectations in cardiac surgery patients is moderated by level of disability: the successful development of a brief psychological intervention. Psychol Health Med. 2016; 21(3): 272-285.

60. Waljee J, McGlinn EP, Sears ED, et al. Patient expectations and patient-reported outcomes in surgery: a systematic review. Surgery. 2014; 155(5): 799-808.

61. Tefikow S, Barth J, Maichrowitz S, et al. Efficacy of hypnosis in adults undergoing surgery or medical procedures: a meta-analysis of randomized controlled trials. Clin Psychol Rev. 2013; 33(5): 623-636.

62. Bradt J, Dileo C, Shim M Music interventions for preoperative anxiety. Cochrane Database Syst Rev. 2013(6): CD006908.

63. Hansen MV, Halladin NL, Rosenberg J, et al. Melatonin for pre- and postoperative anxiety in adults. Cochrane Database Syst Rev. 2015(4): CD009861.

64. Shelley M, Pakenham $\mathrm{K}$ The effects of preoperative preparation on postoperative outcomes: the moderating role of control appraisals. Health Psychol. 2007; 26(2): 183-191.

65. Hoogeboom TJ, Dronkers JJ, Hulzebos EH, et al. Merits of exercise therapy before and after major surgery. Curr Opin Anaesthesiol. 2014; 27(2): 161-166.

66. Hulzebos EH, Smit Y, Helders PP, et al. Preoperative physical therapy for elective cardiac surgery patients. Cochrane Database Syst Rev. 2012; 11: CD010118.

67. Ditmyer MM, Topp R, Pifer M Prehabilitation in preparation for orthopaedic surgery. Orthop Nurs. 2002; 21(5): 43-51; quiz 52-44.

68. Henschke N, Diong J Exercise reduces pain and improves physical function for people awaiting hip replacement surgery. Br J Sports Med. 2014; 48(6): 477-478

69. Mishriky BM, Waldron NH, Habib AS Impact of pregabalin on acute and persistent postoperative pain: a systematic review and meta-analysis. Br J Anaesth. 2015; 114(1): 10-31.

70. Alayed N, Alghanaim N, Tan X, et al. Preemptive use of gabapentin in abdominal hysterectomy: a systematic review and meta-analysis. Obstet Gynecol. 2014; 123(6): 1221-1229.

71. Carroll I, Hah J, Mackey S, et al. Perioperative interventions to reduce chronic postsurgical pain. J Reconstr Microsurg. 2013; 29(4): 213-222. 


\section{Chapter 7}

72. Clarke $H$, Bonin RP, Orser BA, et al. The prevention of chronic postsurgical pain using gabapentin and pregabalin: a combined systematic review and meta-analysis. Anesth Analg. 2012; 115(2): 428-442.

73. Schmidt PC, Ruchelli G, Mackey SC, et al. Perioperative gabapentinoids: choice of agent, dose, timing, and effects on chronic postsurgical pain. Anesthesiology. 2013; 119(5): 1215-1221.

74. Chaparro LE, Smith SA, Moore RA, et al. Pharmacotherapy for the prevention of chronic pain after surgery in adults. Cochrane Database Syst Rev. 2013(7): CD008307.

75. de Meij N, van Grotel M, Patijn J, et al. First Dutch Consensus of Pain Quality Indicators for Pain Treatment Facilities. Pain Pract. 2016; 16(1): 57-66.

76. Dukeshire S, Gilmour D, MacDonald N, et al. Development and evaluation of a web site to improve recovery from hysterectomy. Comput Inform Nurs. 2012; 30(3): 164-175; quiz 176-167.

77. Kleinbeck SV Self-reported at-home postoperative recovery. Res Nurs Health. 2000; 23(6): 461-472.

78. Verhagen AP, Downie A, Popal N, et al. Red flags presented in current low back pain guidelines: a review. Eur Spine J. 2016; 25(9): 2788-2802. 


\section{Valorization}

The aim of this paragraph is to describe how the "academic wisdom", as obtained by the studies described in this thesis, can be translated to societal benefit. There are several domains where benefit from the study findings can be expected. In the first place there is the potential impact on the target population of this dissertation, surgical patients. More in general, the societal and economic value can be considered. Finally, by this thesis new scientific insights have been obtained, and it may generate new scientific initiatives. These issues will be described in this order below.

\section{THE IMPACT ON THE SURGICAL PATIENT.}

The most important aspect of this thesis is reflected by the call for further optimization of perioperative (preventive) treatment, aiming at the reduction of the impact of established risk factors of poor postoperative quality of life. Further optimization is necessary, notwithstanding the fact that for hysterectomy, similar to other surgery types, improvements in care pathways have been implemented continuously. Over the last decade, many of the publications on this subject focused on implementation of enhanced recovery pathways, also with regard to hysterectomy, as reflected by the comprehensive review of Kalogera and Dowdy. ${ }^{1}$ To achieve an enhanced recovery after surgery (ERAS), meaning an earlier return of normal bowel function and a shorter length of stay, with a stable complication rate compared with previous care paths, a combination of pre-, per-, and postoperative interventions is performed. The preoperative interventions (I) concern patient counseling and education, preoperative diet, bowel preparation, and use of preemptive analgesia. An important intraoperative intervention (II) is the application of anesthesia types that allow for rapid awakening, decreased systemic opioid requirements, and prevention of postoperative nausea and vomiting (PONV). Other objectives are to maintain normothermia and normovolumia and to avoid nasogastric tubes and peritoneal drains. Postoperative interventions (III) aim at continuation of the normovolemic status, early postoperative feeding and mobilization, early urinary catheter removal, prevention of ileus, and application of multimodal analgesia. ${ }^{1}$ Particularly for two of these aspects, namely preoperative counseling and education, and peri- 
operative pain treatment, the results of this thesis are indicative for further fine tuning of perioperative care.

In the first place, preoperative counseling and education as described in the ERAS protocol aim at informing the patient about the procedure and how patients can contribute to their own healing process. However, the ERAS protocol seems not to differentiate between patients with different psychosocial characteristics. Based on the findings that preoperative anxiety and catastrophizing are risk factors of chronic postoperative pain after different types of surgery, and based on the establishment of other psychosocial predictors of poor postoperative quality of life after hysterectomy besides surgical worries, this thesis adds support to the idea that preoperative patient preparation should be more tailor made. To achieve this, propositions are made for a more comprehensive preoperative screening, including psychosocial measures. In addition, a quick scan of existing literature on treatment options for the established risk factors is described in the discussion: several promising findings are reported, in particular for music listening and hypnosis. In the end, patients with an increased preoperative psychosocial risk profile might benefit from a better postoperative quality of life because of early detection and treatment of established risk factors.

The second issue from the ERAS protocol that also is a subject of this thesis is perioperative pain treatment. Concerning the preoperative interventions (I) with regard to pain, ERAS promotes the start of preemptive analgesia. In this thesis a call was made for studies on more extended or aggressive treatment of preoperative pain. Because preoperative pain is a known risk factor of acute and chronic postsurgical pain, it would be worth studying whether preemptive analgesia starting earlier than 24 hours before surgery could be effective. Until now such studies are rare. Another aspect that might be helpful for future patients is the implementation of transitional pain service (TPS) teams as proposed by Clarke et al. ${ }^{2}$ Implementing a preoperative screening to detect patients with increased pain-related risks, and subsequently, the application of preoperative education and a tailor-made treatment plan, do perfectly match the conclusions of this thesis and the current opinion favoring a more patient-centered health care. Intraoperative analgesic treatment (II) will also affect the acute postoperative period. The results of this thesis revealed that among the hysterectomy patients no less than $42 \%$ indicated an NRS pain score $\geq 4$ one hour after arrival at the PACU. This once more underlines the necessity of further improvements to achieve in the reduction of acute postoperative pain. Several suggestions are done, concerning (re-) consideration of treatment options such as gabapentin, pregabalin, ketamine, patient-controlled analgesia, or trunk blocks. Some of these options are meant to be started intraoperatively. Concerning the postoperative analgesic interventions (III) ERAS also proposes multimodal pharmacological analgesia in combination with regional analgesia. Patients may benefit if the suggested TPS teams are implemented including postdischarge follow-up. In addition, e-health technology could be supportive in terms of information supply for discharged patient, and by using interactive systems patients can enter their actual 
health status, facilitating postdischarge monitoring. These measures could be beneficial to patients postoperative quality of life. However, $39 \%$ indicated an NRS pain score $\geq 4$ at postoperative day four: the implementation of postdischarge follow-up including ehealth monitoring might not be sufficient for all of these patients. Therefore, the principle of early discharge as promoted by ERAS might need reconsideration for a subset of patients at risk of an impaired recovery, who perhaps might be better off with a prolonged, intensive, clinical postoperative treatment.

One of the aims according to ERAS is early mobilization after surgery. Early mobilization was not explicitly assessed for this thesis, however, physical function was an important measure at baseline and 3 and 12 months follow-up. Baseline physical functioning was a significant predictor of physical functioning at follow-up. Therefore, also for physical functioning preoperative assessment and if necessary, prehabilitation interventions might be of benefit for patients with a poor baseline physical function level. In summary, concerning several aspects of the ERAS pathway, this thesis adds suggestions for improvement or fine tuning. Some of these suggestions are easier to implement than other, for some additional evidence is needed.

In this thesis detailed information concerning the pre-and postoperative status of hysterectomy patients is described. It is reassuring for women considering a hysterectomy that in general postoperative quality of life is high, in many cases pain is reduced significantly, the risk of postoperative depression is not increased because of the surgery, and feelings of femininity are not affected. On the other hand, especially women with a poor preoperative physical or mental condition should notice that hysterectomy will probably not resolve all pre-existing problems and where possible, preoperative optimization might be helpful to achieve improvements in quality of life.

The findings with regard to the predictors of postoperative quality of life are based on a hysterectomy population. Therefore conclusions for other surgery populations cannot be drawn. However, many of the results are in line with previous findings on populations undergoing other types of surgery. And for the predictors anxiety and catastrophizing in relation to chronic postsurgical pain also a literature review was performed among patients undergoing various types of surgery. Therefore, not only hysterectomy patients can benefit from these results, but also patients who have to undergo other types of clinical and day surgery.

Knowledge of surgical fear as a risk factor for CPSP is important for patients because it is supposed to result in more attention for preventive measures on this issue which in turn, should lead to improved postoperative outcome. In addition, this thesis revealed that patients undergoing day surgery not necessarily are less fearful when compared to clinical surgery patients. This is relevant information when preoperative preparation for day surgery patients is discussed. Also the impact of other risk factors is further established or confirmed. 


\section{SOCIETAL AND ECONOMIC RELEVANCE}

With the established validity of the SFQ an instrument has become available for the assessment of surgical fear among adult clinical and day surgery patients. The SFQ is sensitive to detect even small differences in surgical fear based on time course and other causes. It provides insight in fear of short- and long-term consequences of surgery, and if necessary even on more detailed (item) level. It is available for free in Dutch, English, Portuguese, and Brazilian Portuguese. Moreover, translations in several other languages are in preparation: Bengali, Czech, Italian, Malay, Serbian, Slovak, and Turkish. The SFQ can be used across many countries and it supports the identification of an important preoperative risk factor, which is the first step towards preventive measures.

The vast grow in number of diagnostic and treatment options for many diseases is accompanied by an enormous grow in medical cost. In many countries, the control of increasing costs for health care facilities has become an important issue. As a result many programs aiming at improved efficiency are implemented. Against the background of this, poor postoperative quality of life can be considered as an unwanted and inefficient outcome of surgery. The impact of postoperative pain and other aspects of poor postoperative recovery has been extensively described in many publications: it is associated with huge personal and societal burden, medical costs, social costs, and economic loss. ${ }^{3-8}$ Considering the large number of surgeries that take place every day, even the smallest improvement in care pathways may have large beneficial effects for the society. Therefore, increased insight in factors associated with postoperative quality of life leading to implementation of improvements in perioperative care as proposed in this thesis, can lead to improvements in postoperative quality of life. In turn, this may have a positive impact on society in terms of medical and social cost savings, less absenteeism, and a healthier population.

\section{SCIENTIFIC RELEVANCE}

The finding that anxiety and catastrophizing play a role in the development of CPSP resulted in recommendations to include these measures in future studies on postoperative pain. In addition, aggregate scores were used for the assessment of psychological measures with a certain overlap between the underlying psychological constructs. In line with previous studies the results further confirm that these aggregate scores are well usable. Moreover, the use of these aggregate scores may trigger the discussion whether, for the assessment of preoperative vulnerability and resilience, detailed multiple scales, partly overlapping, are always necessary.

When it comes to the selection of one or more specific scales for the assessment of preoperative fear, it is important to realize that after studying different measures of 
preoperative fear, it became apparent that the TSK seems not of great value for the prediction of chronic postoperative pain. Preferably other measures of fear should be used instead.

For the assessment of surgical fear, at least in cataract surgery patients, it became obvious that surgical fear can be assessed at any time point within the week before surgery without significantly affecting the results, with the exception of certain subgroups as mentioned in chapter 4 . Furthermore it was established that before cataract surgery $8.6 \%$ of the patients would have preferred a preoperative treatment of surgical fear and $14 \%$ of the patients selected one of the suggested options for the preoperative treatment of fear. Considering the fact that surgical fear levels before cataract surgery are lower compared with other types of surgery it might be speculated that for more invasive surgery the proportion of patients that would prefer any fear treatment will even be larger. Therefore, the suggestion for preoperative optimization does not only origin from results of prediction analyses, but is supported by patient preferences as expressed in this thesis.

Whether there is an association between preoperative levels of fear and cortisol and alpha amylase or not, remains an interesting research question for study on patients planned for surgery eliciting higher fear levels than cataract surgery.

The validation of prediction models is still ongoing. Previously studies on prediction models and their validation have been published, e.g. Kalkman et al., Stessel et al. ${ }^{9,}{ }^{10}$ In the future these models will probably be expanded with genetic variants. These data were also collected in our hysterectomy population.

The most relevant scientific aspect is the call for maximum scientific support for studies answering questions with regard to the selection and implementation of the most adequate assessment tools and preventive measures, aiming at improving postoperative quality of life. At this moment only associations between predictors and outcomes are established. However, the proof that there is a causal link between e.g. fear, and pre- and acute postoperative pain and long-term recovery can only be established by intervention studies. Understanding these links is not only important for patients, but also in a scientific sense. 


\section{REFERENCES}

1. Kalogera E, Dowdy SC Enhanced Recovery Pathway in Gynecologic Surgery: Improving Outcomes Through Evidence-Based Medicine. Obstet Gynecol Clin North Am. 2016; 43(3): 551-573.

2. Clarke $\mathrm{H}$, Poon $\mathrm{M}$, Weinrib $\mathrm{A}$, et al. Preventive analgesia and novel strategies for the prevention of chronic post-surgical pain. Drugs. 2015; 75(4): 339-351.

3. Crombie IK, Davies HT, Macrae WA Cut and thrust: antecedent surgery and trauma among patients attending a chronic pain clinic. Pain. 1998; 76(1-2): 167-171.

4. Goren A, Mould-Quevedo J, daCosta DiBonaventura M Prevalence of pain reporting and associated health outcomes across emerging markets and developed countries. Pain Med. 2014; 15(11): 18801891.

5. Langley P, Muller-Schwefe G, Nicolaou A, et al. The societal impact of pain in the European Union: health-related quality of life and healthcare resource utilization. J Med Econ. 2010; 13(3): 571-581.

6. Langley P, Perez Hernandez C, Margarit Ferri C, et al. Pain, health related quality of life and healthcare resource utilization in Spain. J Med Econ. 2011; 14(5): 628-638.

7. Langley PC The societal burden of pain in Germany: health-related quality-of-life, health status and direct medical costs. J Med Econ. 2012; 15(6): 1201-1215.

8. Takura T, Ushida T, Kanchiku T, et al. The societal burden of chronic pain in Japan: an internet survey. J Orthop Sci. 2015; 20(4): 750-760.

9. Kalkman CJ, Visser K, Moen J, et al. Preoperative prediction of severe postoperative pain. Pain. 2003; 105(3): 415-423.

10. Stessel B, Fiddelers AA, Marcus MA, et al. External Validation and Modification of a Predictive Model for Acute Postsurgical Pain at Home after Day Surgery. Clin J Pain. 2016. 
Summary 
When people undergo curative surgery, their aim is to achieve an increased level of quality of life after surgery, or at least maintain their preoperative level of quality of life. However, postoperative quality of life is determined by many factors. A clear insight in these factors can help with predicting postoperative outcome and, more importantly, it can serve as starting point for targeted perioperative preventive measures aiming at higher quality of life after surgery. A well-known psychological issue in surgical patients is preoperative fear. This thesis starts with the description of three studies on the impact and the measurement of surgical fear. Thereafter, based on a multicenter observational study on elective hysterectomy patients with a benign indication, predictors of pain, physical function, global surgical recovery, depression, and well-being are addressed. In addition, epidemiological data on baseline status and the multiple outcome measures are presented.

Anxiety and pain catastrophizing are known predictors of acute postoperative pain. However, it is not well established whether they also predict chronic postsurgical pain (CPSP). The aim of the systematic review and meta-analysis described in chapter 2 , was to investigate whether high levels of preoperative anxiety or pain catastrophizing are associated with an increased risk of CPSP. A literature search was performed using the electronic databases PubMed and PsycINFO. Additional literature was obtained by reference tracking and expert consultation. Studies from 1958 until October 2010, investigating the association between preoperative anxiety or pain catastrophizing and CPSP in adult surgery patients, were assessed. The primary outcome was the presence of pain at least 3 months postoperatively. Twenty-nine studies were included for review in which 14 instruments were used to assess anxiety or pain catastrophizing. Sixteen studies (55\%) reported a statistically significant association between anxiety or pain catastrophizing and CPSP. The proportion of studies reporting a statistically significant association was $67 \%$ for studies of musculoskeletal surgery and $36 \%$ for other types of surgery. There was no association with study quality, but larger studies were more likely to report a statistically significant relationship. The overall pooled odds ratio, on the basis of 15 studies, ranged from 1.55 to 2.10. Pain catastrophizing might be of higher predictive utility compared with general anxiety or more specific pain-related anxiety. We concluded that there is evidence that anxiety and catastrophizing play a role in the development of CPSP. We recommend that anxiety measures should be incorporated in future studies investigating the prediction and transition from acute to chronic postoperative pain.

As shown in chapter 2, a diversity of instruments exists to assess anxiety and fear. Because only a limited number of instruments is available for the assessment of surgical fear and existing instruments are either limited in scope, or aiming at specific patient groups, there is need for an instrument which is suitable for a broad scope of patients and surgery types, and which is easy to obtain in view of a general clinical use in the future. Therefore the Surgical Fear Questionnaire (SFQ) was developed. The SFQ is an instrument dedicated to detect fear of surgery in adult patients across different types of 
surgery. In chapter 3 the validity and reliability of the SFQ is assessed. Based on existing literature and expert consultation the ten-item SFQ was composed. Data on the SFQ were obtained from five prospective studies $(N=3233)$ in inpatient or day surgery patients. These data were used for exploratory factor analysis (EFA), confirmatory factor analysis (CFA), reliability analysis and validity analysis. EFA in Study 1 and 2 revealed a two-factor structure with one factor associated with fear of the short-term consequences of surgery (SFQ-s, item 1-4) and the other factor with fear of the long-term consequences of surgery (SFQ-I, item 5-10). However, in both studies two items of the SFQ-I had low factor loadings. Therefore in Study 3 and 4 the two-factor structure was tested and confirmed by CFA in an eight-item version of the SFQ. Across all studies significant correlations of the SFQ with pain catastrophizing, state anxiety, and preoperative pain intensity indicated good convergent validity. Internal consistency (Cronbach's alpha) was between 0.765-0.920 (SFQ-total), 0.766-0.877 (SFQ-s), and 0.628-0.899 (SFQ-I). The SFQ proved to be sensitive to detect differences based on age, sex, education level, employment status and preoperative pain intensity. In conclusion, the SFQ is a valid and reliable eight-item index of surgical fear consisting of two subscales: fear of the short-term consequences of surgery and fear of the long-term consequences. However, in all studies that provided data for the validation process described in chapter 3 , the SFQ was assessed only once. As a result, the sensitivity to differences in fear level over time is unknown. Also the convergent validity with biological indices of stress is unknown. These were reasons to perform another study.

In chapter 4 additional steps in the validation process of the SFQ are described. In cataract surgery patients, the course of surgical fear in the week before cataract surgery was assessed, and simultaneously samples of salivary cortisol and alpha-amylase were collected. Aims were to assess the convergent validity of the SFQ with other self-report instruments and biological indices of stress, to examine predictors of the level and time course of fear, and to assess preferences for fear treatment. In a prospective observational cohort study SFQ short-term (SFQ-S) and long-term (SFQ-I) scores were assessed one week, one day, and the morning before cataract surgery, together with salivary cortisol and alpha-amylase (SAA) levels, and numeric rating scale (NRS) fear score. SFQscores were also assessed before second eye surgery. Expected pain and recovery, and sociodemographic and medico-psychological predictors of fear were assessed at baseline. Data of 98 patients were analyzed. The scores of both SFQ-subscales (range 0-40) were generally low, all mean $\leq 9.0$. SFQ-S and SFQ-I correlated significantly with the other self-report instruments: NRS fear 0.83 and 0.89 , expected pain 0.49 and 0.54 , expected recovery -0.27 and -0.44 . No association was found between SFQ-scores and cortisol or SAA level. Predictors of the level of fear were baseline pain and stress. Additional effects of time were found for subgroups based on educational level, antidepressant use, and presurgical stress (SFQ-I). SFQ-scores were significantly lower before the second cataract surgery than before the first, and higher in patients who would have appreciated treatment of fear. In conclusion, convergent validity of the SFQ with other 
self-report measures is shown and the sensitivity of the SFQ permits the detection of small variations in fear caused by time or other factors.

The study described in the chapters 5 and 6 aimed to establish predictors of postoperative quality of life after hysterectomy for a benign indication. Besides surgical fear also other predictors were assessed. One important aspect of surgical recovery is chronic postsurgical pain (CPSP). Chapter 5 describes predictors and epidemiological data of CPSP, and also of physical functioning (SF-36PF, 0-100) and global surgical recovery (global surgical recovery index, 0-100\%), 3 and 12 months after hysterectomy. Data were obtained using a prospective multicenter cohort study. Sociodemographic, somatic, and psychosocial data were assessed in the week before surgery, postoperatively up to day 4, and at 3- and 12-month follow-up. Generalized linear model (CPSP) and linear mixed model analyses (SF-36PF and global surgical recovery index) were used. Baseline data of 468 patients were collected, 412 (88\%) patients provided data for 3-month evaluation and 376 (80\%) patients for 12-month evaluation. After 3 and 12 months, the prevalence of CPSP (numeric rating scale $\geq 4$, scale $0-10$ ) was $10.2 \%$ and $9.0 \%$, respectively, SF-36PF means (SD) were 83.5 (20.0) and 85.9 (20.2), global surgical recovery index $88.1 \%$ (15.6) and 93.3\% (13.4). Neuropathic pain was reported by 20 (5.0\%) patients at 3 months and 14 (3.9\%) patients at 12 months. Preoperative pain, surgeryrelated worries, acute postsurgical pain on day 4 , and surgery-related infection were significant predictors of CPSP. Baseline level, participating center, general psychological robustness, indication, acute postsurgical pain, and surgery-related infection were significant predictors of SF-36PF. Predictors of global surgical recovery were baseline expectations, surgery-related worries, American Society of Anesthesiologists classification, type of anesthesia, acute postsurgical pain, and surgery-related infection. In sum, several predictors were identified for CPSP, physical functioning, and global surgical recovery. Some of the identified factors are modifiable and optimization of patients' preoperative pain status and psychological condition as well as reduction of acute postsurgical pain and surgery-related infection may lead to improvement of outcome.

In chapter 6 the risk and protective factors for depression and well-being, and the prevalence of depression, level of well-being, and feelings of femininity are assessed. Outcome measures were the Center for Epidemiological Studies-Depression scale (CES$D)$, the 12-item well-being questionnaire (W-BQ 12) energy and positive well-being subscales, and feelings of femininity. Linear mixed model analyses were performed. The levels of depression, energy, and positive well-being after hysterectomy were predicted by their corresponding baseline levels and by baseline pain. Postoperative infection reported at 12 months affected CES-D and energy level. Several other gynecological, psychosocial, or perioperative factors were also predictive for one of the outcomes. Prevalence of depression at baseline, 3 and 12 months was 24\%, 19\%, and 21\%, respectively. In general, well-being scores were slightly higher 3 and 12 months after hysterectomy than at baseline. Feelings of femininity were not negatively affected in $92 \%$ of the 
patients. It was concluded that optimizing the preoperative psychosocial status, intensified perioperative pain treatment, and reduction of postoperative infection may further improve psychological outcome after hysterectomy. In the majority of patients we observed small but significant improvements with regard to postoperative depression and well-being, while feelings of femininity were unaffected.

In chapter 7, the findings of the previous chapters are discussed and mutual relationships are considered. Implications for practice and future research are described. It was concluded that in order to improve postoperative quality of life, careful implementation of several perioperative preventive measures and treatment options, in a multidisciplinary approach, is mandatory. 

Samenvatting 
Als mensen een curatieve operatie ondergaan doen ze dat om de kwaliteit van leven te verbeteren, of ten minste het bestaande niveau te behouden. Kwaliteit van leven wordt bepaald door vele factoren. Inzicht in deze factoren kan behulpzaam zijn bij het voorspellen van het resultaat van een operatie. Bovendien kan deze informatie dienen als uitgangspunt voor doelgerichte, perioperatieve, preventieve maatregelen ter verhoging van de kwaliteit van leven na de operatie. Een herkenbaar psychologisch fenomeen voor operatiepatiënten is preoperatieve angst. Deze dissertatie begint met de beschrijving van drie studies die handelen over de impact en het meten van preoperatieve angst. Vervolgens worden voorspellende factoren van pijn, lichamelijk functioneren, depressie en welbevinden beschreven, gebaseerd op een observationeel onderzoek onder vrouwen die een electieve baarmoederverwijdering ondergaan vanwege een goedaardige indicatie. Epidemiologische gegevens ten aanzien van de preoperatieve uitgangssituatie, alsmede de diverse uitkomstmaten worden eveneens weergegeven.

Angst en pijn catastroferen zijn bekende voorspellers van acute postoperatieve pijn. Minder duidelijk is of zij ook voorspellend zijn voor chronische postoperatieve pijn (CPOP). Het doel van het systematische literatuuronderzoek en de meta-analyse zoals beschreven in hoofdstuk 2, was om te onderzoeken of hogere niveaus van preoperatieve angst of pijn catastroferen geassocieerd zijn met een verhoogd risico op CPOP. Met behulp van de elektronische databases PubMed en PsycINFO werd relevante literatuur gezocht. Aanvullende publicaties werden verkregen middels het doorzoeken van referentielijsten en het raadplegen van deskundigen. Studies gepubliceerd tussen 1958 en oktober 2010, die het verband tussen preoperatieve angst of pijn catastroferen en CPOP onderzochten bij volwassenen, werden beoordeeld. De primaire uitkomstmaat was aanwezigheid van pijn tenminste 3 maanden na een operatie. Er werden 29 publicaties geïncludeerd en geëvalueerd, waarin 14 meetinstrumenten voor het meten van angst en pijn catastroferen werden gebruikt. Er waren 16 publicaties (55\%) die een statistisch significant verband tussen angst of pijn catastroferen en CPOP rapporteerden. De proportie van studies die een significant verband rapporteerden was $67 \%$ bij publicaties over musculoskeletale operaties en 36\% bij publicaties over andere operaties. De kwaliteit van de geëvalueerde studies had geen invloed op de bevindingen, maar in studies met een grotere populatie was de kans op een statistisch significant verband groter. De gepoolde odds-ratio, gebaseerd op 15 publicaties, liep uiteen van 1,55 tot 2,10. Het lijkt erop dat pijn catastroferen mogelijk een grotere voorspellende waarde heeft dan algemene angst of pijngerelateerde angst. De conclusie was dat er bewijs is dat angst en pijn catastroferen een rol spelen bij het ontstaan van CPOP. Wij raden aan om angst als factor mee te nemen in toekomstige studies die zich richten op het voorspellen van pijn alsook in studies die zich richten op de transitie van acute naar chronische postoperatieve pijn.

Zoals blijkt uit hoofdstuk 2, zijn er vele vragenlijsten voor het meten van vrees of angst. Maar voor het meten van preoperatieve angst is maar een beperkt aantal vragenlijsten beschikbaar en deze zijn ofwel beperkt in reikwijdte ofwel bedoeld voor spe- 
cifieke patiëntengroepen. Dit betekent dat er ruimte was voor een vragenlijst, geschikt voor een breed scala aan operatiepatiënten. Met het oog op toekomstige klinische toepassing, moet het instrument makkelijk zijn in gebruik. Voor dit doel werd de Vragenlijst Vrees voor de Operatie (VVO) ontwikkeld. De VVO is een vragenlijst gericht op het meten van preoperatieve angst, bij volwassenen, voor diverse operaties. In hoofdstuk 3 is beschreven hoe de validiteit en betrouwbaarheid van de VVO is vastgesteld. Gebaseerd op bestaande literatuur en deskundigenadvies was de 10-item VVO samengesteld. Vervolgens werden data, verkregen uit 5 prospectieve studies ( $N=3233$ ) met zowel klinische als dagcentrumpatiënten, gebruikt voor exploratieve (EFA) en confirmatieve (CFA) factor analyses alsook voor het testen van de betrouwbaarheid en validiteit. EFA met de data van de studies 1 en 2 lieten een 2-factor structuur zien, waarin één factor verband houdt met angst voor de korte termijn aspecten (VVO-k, item 1-4) van de operatie en de andere factor verband houdt met de lange termijn aspecten (VVO-I, item 5-10). Echter, in beide studies kwamen 2 items naar voren met een lage factor lading. Daarom werd de 2-factor structuur onderzocht en bevestigd met de data van studie 3 en 4, middels een CFA uitgevoerd op een 8-item versie van de VVO. Een goede convergente validiteit werd weerspiegeld door significante correlaties, in alle studies, tussen de VVO en pijn catastroferen, situationele angst, en preoperatieve pijn intensiteit. De interne consistentie (Cronbach's alfa) was 0,765-0,920 (VVO-totaal score), 0,766-0,877 (VVO-k) en 0,628-0,899 (VVO-I). Het bleek dat de VVO gevoelig genoeg was om verschillen op basis van leeftijd, geslacht, opleidingsniveau, werkstatus en preoperatieve pijnintensiteit te kunnen onderscheiden. Concluderend, de VVO is een 8-item vragenlijst voor het meten van preoperatieve angst, bestaande uit 2 subschalen: angst voor de korte termijn aspecten en angst voor de lange termijn aspecten van de operatie. Echter, alle studies beschreven in hoofdstuk 3 hebben de VVO maar op één tijdstip voor de operatie gemeten. Op grond hiervan kunnen er geen uitspraken worden gedaan over de gevoeligheid van de VVO voor veranderingen van preoperatieve angst in de tijd. Ook de convergente validiteit in relatie tot biologische indicatoren van stress is niet bekend. Dit waren 2 redenen om nog een vervolgstudie uit te voeren.

Hoofdstuk 4 beschrijft de vervolgstappen die zijn ondernomen in het validatieproces van de VVO. Bij patiënten die een cataractoperatie moesten ondergaan werd het beloop van angst in de week voor de operatie gemeten. Ook werden er speekselmonsters afgenomen ter bepaling van cortisol en alfa-amylase spiegels. Doel van de studie was om de convergente validiteit met andere patiënt-gerapporteerde meetinstrumenten en met biomarkers van stress te onderzoeken en verder ook het vaststellen van voorspellers van de hoogte en het beloop van angst en tenslotte, voorkeuren van patiënten voor een eventuele behandeling van preoperatieve angst vast te stellen. In een prospectieve observationele cohortstudie werd de angst voor de korte termijn aspecten (VVO-k) en de lange termijn aspecten (VVO-I) van de operatie gemeten op de volgende tijdstippen: een week voor de operatie, een dag voor de operatie en de ochtend voor de operatie. Tegelijkertijd werden speekselmonsters voor cortisol en alfa-amylase afgenomen alsook 
een numeric rating scale (NRS) voor angst. VVO-scores werden ook afgenomen voor de tweede operatie, van het andere oog. Een week voor de operatie werden tevens verwachtingen ten aanzien van postoperatief herstel en pijn geïnventariseerd alsmede sociodemografische en psychomedische voorspellers van angst. De gegevens van 98 patiënten werden geanalyseerd. De scores op beide subschalen van de VVO (schaal 040) waren over het algemeen laag, gemiddeld $\leq 9,0$. De VVO-k en VVO-I vertoonden significante correlaties met de andere patiënt-gerapporteerde meetinstrumenten: NRS angst 0,83 en 0,89, verwachtte pijn 0,49 en 0,54, verwacht herstel -0,27 en -0,44. Er werd geen verband gevonden tussen $\mathrm{VVO}$-scores en cortisol en alfa-amylase spiegels. Voorspellende factoren voor de hoogte van angst waren pijn voor de operatie en stress. Effecten van tijd op de angst werden gevonden voor subgroepen gebaseerd op opleidingsniveau, gebruik van antidepressiva, en (alleen voor VVO-I) preoperatieve stress. De VVO-scores waren significant lager voor de tweede operatie dan voor de eerste en hoger bij patiënten die aangaven dat ze een behandeling voor preoperatieve angst op prijs gesteld zouden hebben. Concluderend, de convergente validiteit van de VVO met andere patiënt-gerapporteerde meetinstrumenten is aangetoond en de sensitiviteit van de VVO blijkt sterk genoeg om kleine variaties in angst ten gevolge van tijdsverloop of andere factoren te kunnen registreren.

De studie die beschreven wordt in de hoofdstukken 5 en 6 heeft als doel het vaststellen van voorspellers van de kwaliteit van leven bij vrouwen die een baarmoederverwijdering kregen vanwege een goedaardige indicatie. Behalve preoperatieve angst worden ook andere voorspellers onderzocht. Een belangrijk aspect van postoperatieve kwaliteit van leven betreft chronische postoperatieve pijn (CPOP). In hoofdstuk 5 worden voorspellers en epidemiologische bevindingen ten aanzien van CPOP beschreven, maar ook van lichamelijk functioneren (SF-36PF, 0-100) en globaal postoperatief herstel (GSR, 0-100\%), 3 en 12 maanden na de operatie. Onderzoeksgegevens werden verkregen middels een prospectieve observationele cohortstudie. Sociodemografische, somatische en psychosociale gegevens werden verzameld in de week voor de operatie, van de operatiedag tot/met dag vier en 3 en 12 maanden na de baarmoederverwijdering. Generalized linear model (CPOP) en Linear mixed model analyses (SF-36 PF en GSR) werden toegepast. Baselinegegevens van 468 patiënten werden verzameld, 412 (88\%) patiënten leverden data voor de 3-maanden analyse en 376 (80\%) voor de 12-maanden analyse. Na 3 en 12 maanden was de prevalentie van CPOP (pijn NRS $\geq 4$, schaal 0-10) respectievelijk 10,2\% en 9,0\%, SF-36PF gemiddelde (SD) was 83,5 $(20,0)$ en $85,9(20,2)$ en GSR $88,1 \%(15,6)$ en $93,3 \%(13,4)$. Neuropathische pijn werd gerapporteerd door 20 $(5,0 \%)$ patiënten na 3 maanden en $14(3,9 \%)$ patiënten na 12 maanden. Preoperatieve pijn, operatie-gerelateerde zorgen, acute postoperatieve pijn op dag 4 en operatiegerelateerde infecties waren significante voorspellers van CPOP. Lichamelijk functioneren voor de operatie, het ziekenhuis waar de operatie plaatsvond, algemene psychologische robuustheid, de indicatie voor de baarmoederverwijdering en acute postoperatieve pijn waren voorspellers voor SF-36PF. Voorspellers van globaal postoperatief her- 
stel waren preoperatieve verwachtingen ten aanzien van het herstel, operatiegerelateerde zorgen, American Society of Anesthesiologists classificatie, anesthesietype, acute postoperatieve pijn, en operatie-gerelateerde infecties. Samengevat, verschillende voorspellers voor CPOP, SF-36PF en GSR werden geïdentificeerd en sommigen van deze voorspellers zijn beïnvloedbaar. Het optimaliseren van de preoperatieve pijnstatus en psychologische conditie, evenals het terugdringen van operatie-gerelateerde infecties zou kunnen leiden tot verdere verbetering van postoperatieve resultaten.

In hoofdstuk 6 werden risico- en beschermende factoren voor depressie en welbevinden, alsmede de prevalentie van depressie, het niveau van welbevinden en gevoelens van vrouwelijkheid onderzocht. Uitkomstmaten waren de Center for Epidemiological Studies-Depression schaal (CES-D), de well-being questionnaire energy en positive well-being subschalen (W-BQ 12) en gevoelens van vrouwelijkheid. Linear mixed model analyses werden toegepast. De mate van depressie, energie, en positief welbevinden na een baarmoederverwijdering werd voorspeld door de preoperatieve uitgangswaarde en preoperatieve pijn. Postoperatieve infectie gerapporteerd na 12 maanden was van invloed op de hoogte van CES-D en energie. Verschillende andere gynaecologische, psychosociale of perioperatieve factoren waren eveneens voorspellend voor een van de uitkomstmaten. De prevalentie van depressie preoperatief en na 3 en 12 maanden was respectievelijk 24\%, 19\% en $21 \%$. In het algemeen waren de scores van welbevinden 3 en 12 maanden na de baarmoederverwijdering enigszins hoger dan voor de operatie. Gevoelens van vrouwelijkheid waren niet negatief beïnvloedt bij 92\% van de patiënten. De conclusie was dat het optimaliseren van de preoperatieve psychologische status, geïntensiveerde perioperatieve pijnbehandeling en reductie van postoperatieve infectie het psychologische herstel na een baarmoederverwijdering verder zou kunnen optimaliseren. In de meerderheid van de patiënten werd een kleine maar significante verbetering gevonden ten aanzien van depressie en welbevinden en gevoelens van vrouwelijkheid werden niet aangetast.

In hoofdstuk 7 worden de bevindingen uit de eerdere hoofdstukken bediscussieerd en in onderlinge samenhang beschouwd. Implicaties voor de praktijk en toekomstig onderzoek worden beschreven. De algemene conclusie luidt dat, om postoperatieve kwaliteit van leven verder te verbeteren, zorgvuldige implementatie van diverse perioperatieve preventieve maatregelen en behandelopties, in multidisciplinair verband, noodzakelijk is. 



\section{Dankwoord}


In een les over wetenschappelijk onderzoek beschreef ik de uitvoering van één studie, van de totstandkoming van het protocol tot, 8 jaar later, de eerste publicatie. Na afloop zei een van de toehoorders: "Hoe kan iemand nu zeggen dat een studie zijn studie is?" Daarmee sloeg hij de spijker op zijn kop.

Beste Marco Marcus, in 2003 kwam er een vacature voor een researchverpleegkundige binnen je onderzoekslijn. Aangezien de interesse voor onderzoek inmiddels gewekt was in de voorafgaande periode was het voor mij een logische stap om hierop te solliciteren. Sinds mijn begin in september 2003 is er veel gebeurd. Je hebt me betrokken bij vele, uiteenlopende projecten en zo heb ik een enorme ervaring in klinisch onderzoek op kunnen bouwen. Daarmee ben je voor mijn loopbaan van essentiële betekenis geweest en sta je aan de basis van dit proefschrift. Je was over het algemeen meer een man van de grote lijnen. Daarbij gaf je blijk van een helder inzicht in de actualiteit én van een vooruitziende blik. Feedback op detailniveau hield je graag beperkt, maar met een enkele opmerking wist je vaak de vinger op de zere plek te leggen. Lange tijd had ik mijn kamer tegenover de jouwe. Terugdenkend hoor ik weer hoe regelmatig mensen lachend je kamer verlieten na een overleg. Welnu, om ook in serieuze zaken enigszins de luchtigheid erin te houden, is een kunst die werk en leven een stuk aangenamer maakt. En daarmee wil ik maar zeggen: ik kijk met veel plezier terug op onze samenwerking.

Beste Madelon Peters, als ik me niet vergis verliep ons eerste contact via de mail, in de trant van: "...als je het over de duivel hebt dan ..." en jouw antwoord "Maar wie is hier nu de duivel? Jij maakt jonge meisjes aan het huilen..." Het betrof enkele vrijwilligsters voor een onderzoek waar ik bij betrokken was, die wachtend op de gang in de buurt van jouw kamer, nogal last hadden van bepaalde bijwerkingen! Gelukkig heeft dit je er niet van weerhouden om later de samenwerking te zoeken in de uitvoering van de hysterectomiestudie. Van het één kwam het ander, en samen met Marco werd uiteindelijk besloten: "Als Maurice die studie gaat uitvoeren kan hij er ook wel zelf op promoveren". En zo stond ik als epidemioloog opeens met één been in de wereld van de anesthesie en met het andere been in de wereld van de psychologie. Voor mij een heel aangename combinatie. Vanaf het begin van het promotietraject heb ik een groot vertrouwen mogen ervaren. Ter zake kundig, was jouw feedback altijd zeer gedetailleerd, genuanceerd en opbouwend. Bovendien reageerde je altijd heel snel, daarmee de vaart erin houdend. Waar mogelijk faciliteerde je met mensen en middelen. Wat kan een promovendus zich nog meer wensen? Het was een voorrecht dit te ondergaan.

Beste Hans Gramke, de tijd van mijn promotie viel grotendeels samen met een voor jou enerverende periode. Een periode die illustreert dat werk, en dus ook "copromotor zijn", niet altijd op de eerste plaats kan (moet) staan. Desondanks heb je een waardevolle bijdrage kunnen leveren aan dit proefschrift maar ook op andere trajecten in mijn reis van verpleegkundige naar wetenschapper. En alle moderne communicatiemiddelen ten spijt, nadat Marco en Madelon hun residentie naar het buitenland verlegden, was het 
fijn om toch nog een lid van het promotieteam in de buurt te hebben die van alles op de hoogte was en die ik laagdrempelig snel even persoonlijk kon raadplegen. Met je combinatie van rust, relativeringsvermogen en deskundigheid waren onze overlegmomenten altijd aangenaam en functioneel tegelijkertijd.

Beste Karin van Ditshuizen, uiteindelijk ben jij het die het vlammetje van mijn wetenschappelijke interesse heeft aangestoken. Toen je als leidinggevende op de recovery kwam wist je al snel diverse medewerkers te binden aan voor hen interessante thema's. Zo begon ik, samen met Cintha en Jos, met literatuuronderzoek om te komen tot meer evidence based handelen. En in een periode dat congresbezoek voor niet-academici toch minder evident was, liet je ons naar een symposium gaan van ACENDIO (Association for Common European Nursing Diagnoses, Interventions and Outcomes) in Wenen. Daar zag ik de "achterkant" van het methodisch werken zoals mij dat op de HBO-V vooral in praktische zin was aangeleerd: de CONCEPTEN die aan de grondslag liggen van ons handelen werden bestudeerd en nieuwe inzichten werden op internationaal niveau bediscussieerd en uitgewisseld. Inspirerend. Vervolgens kon ik als gaststudent de blokken methoden \& technieken van onderzoek en statistiek gaan volgen bij de faculteit gezondheidswetenschappen, de basis om later alsnog de master epidemiologie te doen. Al heeft de projectgroep waarmee het allemaal begon wellicht geen wereldnieuws opgeleverd, het heeft de kiem gelegd voor alle publicaties waar ik later bij betrokken was.

De lokale onderzoekers die de hysterectomiestudie mogelijk hebben gemaakt in de centra buiten het MUMC+. Beste Hans van Suijlekom, het was leuk om na je vertrek uit het MUMC+, op deze manier weer met je samen te kunnen werken. Verzekerd van de onmisbare inzet van Mark Willemsen, Britta de Waal, Lieke Muntinga en Ratna Soekhoe en op het lab Anton van de Stokker, had je de zaken goed voor elkaar in het Catharina ziekenhuis Eindhoven. Beste Jacques Maas, nadat je eerst in het MUMC+ al je bijdrage had geleverd aan de opzet van de studie, hield je de continuïteit erin door ook later in het Máxima Medisch Centrum de hysterectomiestudie weer op te pakken. Beste Anja van Ommering, ik heb grote waardering voor de wijze waarop je de uitvoering van de studie hebt ingepast in je drukke werkzaamheden en studie-activiteiten. Beste Anita Groos, hartelijk dank voor de immer correcte afhandeling van de vele labmaterialen die je voor ons verwerkt hebt. Beste Fleur Tournois, na overleg met Gérard Bremer werd jij de centrale persoon die voor ons de studie in het Zuyderland ziekenhuis Sittard-Geleen (voorheen Orbis Medisch Centrum) ging uitvoeren. Dat deed je samen met de laboranten Cindy Hendrix, Miranda l'Espoir, Marlies Huizinga. Hartelijk dank voor de prettige samenwerking. Mede dankzij de inzet in de drie genoemde ziekenhuizen hebben we in relatief korte tijd de studie kunnen uitvoeren. Diverse andere personen waren op enig moment onmisbaar voor het welslagen van de hysterectomiestudie. Bij het Memic Désirée Jacobs, voor de ondersteuning bij het ontwerpen van de vragenlijsten en het samen met de medewerkers van data entry zorgen voor de digitale omzetting van de 
resultaten. Het was fijn om gebruik te kunnen maken van je kennis van zaken en altijd op korte termijn terecht te kunnen. Beste Sita van Riet, de door jou ontworpen Accessdatabase was onmisbaar om het overzicht te kunnen bewaren bij de logistieke afwikkeling van de in totaal ruwweg 3000 vragenlijsten. En niet te vergeten de mensen van de postkamer, die een belangrijk deel van deze vragenlijsten als in- én uitgaande post op de juiste bestemming hebben gebracht. Binnen het pijnonderzoek is aandacht voor de genetische component nog relatief jong. Daarom was de samenwerking met Jim van Os, Gunter Kenis en Bart Rutten van grote waarde voor het uitwerken en faciliteren van het genetische onderdeel deel van de hysterectomiestudie. Binnenkort zullen de eerste resultaten bekend worden: laten we hopen dat de resultaten ons weer verder kunnen helpen bij het ontginnen van blanke gebieden in het pijnonderzoek. Beste Jeroen Schepers, bedankt voor de tijdige bevoorrading van al het afnamemateriaal. Verder dank ik de medewerkers van de bloedafname van MUMC+ en Berry Soute, Corine Coorens, Claudia Bosman en de overige medewerkers van de BioBank Maastricht, voor de professionele opslag van alle monsters bedoeld voor het genetisch onderzoek.

Van de Universiteitskliniek voor Oogheelkunde Soraya Jonker, Frank vd Biggelaar, Rudy Nuijts, Laura Wielders en het hele team van de poli en dagcentrum OK. Jullie hebben ervoor gezorgd dat de tweede validatiestudie van de Surgical Fear Questionnaire, uitgevoerd bij cataract patiënten, onder zo optimaal mogelijke omstandigheden werd uitgevoerd. Zowel bij de screening, de OK-planning, alsook op de OK-dagen was het een plezier om met jullie samen te werken. Beste Soraya, jij hebt zowel in praktische zin alsook inhoudelijk een belangrijke bijdrage geleverd. Het was fijn om onze kennis met elkaar uit te kunnen wisselen. Ik wens je veel succes met de afronding van jouw proefschrift. Annick Ronden, als student/vrijwilliger heb je grote inzet en betrokkenheid getoond bij je ondersteuning aan dit project. Het kan niet anders dan dat je er de vruchten van zult plukken. Hartelijk dank en veel succes met de afronding van je studie. Dear Clemens Kirschbaum and Sarah Brand of the Biopsychology Laboratory TU Dresden, although we have never met each other, from the beginning it was reassuring to know that the analyses of our salivary samples would be performed under your supervision. Your expertise at this subject, in combination with well-organized logistics resulted in a smooth cooperation.

Voor zover nog niet genoemd, diverse co-auteurs aan de verschillende artikelen. Dear Julie Bruce, it was for my first publication that you became a co-author. I really appreciated the support given by you. By adding your expertise, and being a native English speaker, both the content and the linguistic aspects of the manuscript got an enormous boost. Erik Schouten, hartelijk dank voor het uitvoeren van de confirmatory factor anaIyses en je uitleg bij het duiden van de resultaten. Dear Patrícia Pinto, it was a great pleasure to work with you. Thank you for providing your data for the validation of the Surgical Fear Questionnaire, answering all my questions, and your comments as coauthor. Nancy Nicolson, your input with regard to the study of stress hormones in sali- 
va, as part of the second validation study of the Surgical fear Questionnaire was vital. The selection of the biomarkers, the sampling methods, the selection of the laboratory, the analyses, and the interpretation of the results: thank you for all your advices. Beste Jan Schepers, met een opmerkelijke rust heb je me vele malen bijgestaan bij de diverse statistische analyses. Kritisch en met een frisse blik op de problematiek, vormde jouw bijdrage een belangrijke waarborg voor de kwaliteit van de uitgevoerde statistiek. Beste Dick Schoot, ik kan me nog herinneren dat je spontaan contact opnam om nog een aantal verbeteringen aan het protocol van de hysterectomiestudie toe te voegen. Je betrokkenheid, ook later bij het schrijven van het artikel, waardeer ik zeer.

Diverse personen die tijdens hun wetenschapsstage hun bijdrage hebben geleverd: Anique van Oudheusden, Nieke Oversier, Michiel van Troost, Malou Herman, Nathalie Claassen, Bas Vreeswijk, Veronique Timmer, Heike van Schaik. Jullie hebben als stagiaire een belangrijk deel van het vele werk verzet. Daarmee vormen jullie zowel voor een promovendus maar ook voor een ziekenhuis als organisatie, een niet te miskennen groep.

Medewerkers van de afdeling anesthesiologie onder leiding van Maarten van Kleef en later Wolfgang Buhre. Beste stafleden en assistenten, hartelijk dank voor jullie medewerking bij de diverse studies: de inclusie, het invullen van vragenlijsten en het meedenken bij allerlei zaken. Verder dank ik de medewerkers van de poli voor hun ondersteuning, het waarschuwen als zich weer een onderzoekspatiënt meldde of om mee te denken welke spreekkamer we het beste konden gebruiken. Ook dank ik iedereen van het secretariaat voor alle ondersteuning. De anesthesiemedewerkers op de OK dank ik voor het bijhouden van alle operatiegegevens van de diverse onderzoekspatiënten. Fons Kessels, de door jou ontwikkelde kappacalculator kwam goed van pas in het review artikel. Verder was je niet bij dit proefschrift betrokken, maar ik heb veel van je geleerd op statistisch gebied. Een bijzonder woord van dank voor mijn (oud-)collegaonderzoekers Audrey Fiddelers, José Geurts, Nelleke de Meij, Dianne de Korte en ons hoofd research Bert Joosten. Het is erg fijn om deel uit te kunnen maken van een harmonieus team. Beste Audrey, als kamergenote gedurende het grootste deel van mijn promotie was je niet alleen collega, maar ook mijn belangrijkste sparring partner. Als zodanig heb je, behalve als co-auteur van één artikel, op vele momenten bijgedragen aan het welslagen van dit project. En, samen met José Geurts weet ik mij gesteund door twee geweldige paranimfen.

Bij de uitvoering van de diverse onderzoeken in het MUMC+ kwam ik zelf op een aantal afdelingen. Anton en Marlie, in het bijzonder op verpleegafdeling A2 viel het mij als "bezoeker" iedere keer weer op: de sfeer en de bereidwilligheid waarmee het team onze onderzoeken faciliteerde. Maar ook op verpleegafdeling $\mathrm{C2}$, het chirurgisch dagcentrum en natuurlijk bij mijn oud-collega's van de recovery, kon ik altijd terecht: hartelijk dank voor alle medewerking. 
Arno Lataster, tijdens mijn promotie heb ik een aantal malen je afdeling bezocht ter verdieping van mijn anatomische kennis. Het is een genot om je enthousiasme te mogen ervaren.

Mitchell Giebels. Ik ben vereerd met een echte "Giebels" op de omslag. Als jonge (pop)fotograaf heb je reeds vele wereldsterren gefotografeerd: een schitterende carrière, die ongetwijfeld zijn vervolg zal krijgen.

Tenslotte mijn familie en vrienden, te beginnen met mijn moeder. Natuurlijk heb jij de basis gelegd voor wat er van mij geworden is. Samen met mijn veel te jong overleden vader wist je duidelijk te maken wat er echt toe doet in het leven. In een tijd dat het nog niet vanzelfsprekend was -in tegendeel-, om terminaal zieke patiënten thuis te verplegen, deed jij dat wel, met de volste overtuiging. Je wist je gesteund door professionele hulp, van met name je familie en de buurvrouw Jeanne Beckers. Aldus ervoer ik al jong van dichtbij hoe "postoperatieve kwaliteit van leven" vorm kan krijgen, nog lang voordat ik van het concept zelf had gehoord. Nu je zelf de beperkingen van het ouder worden ervaart, wens ik je toe dat je nog een tijd in redelijke gezondheid van je rust mag genieten. Verder dank aan mijn broer Laurent en schoonzus Ineke Groot, voor de ondersteuning en belangstelling, evenals aan alle overige (schoon-)familie en vrienden.

Lieve Anja, 29 jaar zijn we nu samen en dat ik deelgenoot ben geworden van jouw leven heeft vele mooie momenten en ook leuke ontmoetingen gebracht. De laatste jaren heb je me thuis vaak uit de wind gehouden zodat ik me kon richten op mijn studie en later de promotie. Alleen al hiermee heb je je lijfspreuk "wie goed doet, goed ontmoet", meer dan waar gemaakt. Nu breekt er weer een nieuwe fase aan. Het zal zeker de moeite waard zijn! Beste Pieter, de volgende anekdote typeert je helemaal: "Meester, wilt u een pepermuntje?" "Nee, dank je." "Ik zou het toch maar doen..." Op de basisschool al wist je wat je wilde, kon je situaties kernachtig typeren, en daarbij de ander volledig in zijn waarde laten. Dankjewel voor de poëzie die je toevoegt aan ons leven. 


\section{Curriculum Vitae}

Henricus Mauritius Servatius Theunissen was born on September 191962 in Maastricht, the Netherlands. In 1981 he graduated from pre-university education (in Dutch: VWO) at the Sint Maartenscollege Maastricht. After obtaining his nursing degree 5th level at the HBO-V, Gezondheidszorg Academie in Sittard in 1985, he worked as a registered nurse, temporarily at the Psychiatric Hospital Welterhof, Heerlen, later at the University Hospital of Maastricht. After getting involved in research at the Post Anesthetic Care Unit he started in 2003 as a study nurse at the department of Anesthesiology and Pain management (Prof. Dr. M.A.E. Marcus). In 2008 he obtained a Master's Degree in Public Health - Epidemiology at Maastricht University. Now he works as an epidemiologist, focus of the research activities is perioperative medicine. In collaboration with the department of Clinical Psychological Science of Maastricht University (Prof. Dr. M.L. Peters) he performed this PhD project from 2009 to 2017. He is married with Anja Theunissen - van Meijel and has one son, Pieter. 



\section{List of publications}

1. Van Poucke S, Stevens K, Wetzels R, Kicken C, Verhezen P, Theunissen M, Kuiper G, van Oerle R, Henskens $Y$, Lance M: Early platelet recovery following cardiac surgery with cardiopulmonary bypass. Platelets 2016:1-7.

2. Theunissen M, Peters ML, Schepers J, Maas JW, Tournois F, van Suijlekom HA, Gramke HF, Marcus MA: Recovery 3 and 12 months after hysterectomy: epidemiology and predictors of chronic pain, physical functioning, and global surgical recovery. Medicine (Baltimore) 2016, 95(26):e3980.

3. Hamaekers $A E$, van der Beek $T$, Theunissen M, Enk D: Rescue ventilation through a small-bore transtracheal cannula in severe hypoxic pigs using expiratory ventilation assistance. Anesth Analg 2015, 120(4):890-894.

4. Theunissen $M$, Peters ML, Schouten EG, Fiddelers AA, Willemsen MG, Pinto PR, Gramke HF, Marcus MA: Validation of the surgical fear questionnaire in adult patients waiting for elective surgery. PLoS One 2014, 9(6):e100225.

5. Stessel B, Theunissen M, Fiddelers AA, Joosten EA, Kessels AG, Gramke HF, Marcus MA: Controlled-release oxycodone versus naproxen at home after ambulatory surgery: a randomized controlled trial. Curr Ther Res Clin Exp 2014, 76:120-125.

6. Bouman EA, Theunissen M, Kessels AG, Keymeulen KB, Joosten EA, Marcus MA, Buhre WF, Gramke HF: Continuous paravertebral block for postoperative pain compared to general anaesthesia and wound infiltration for major oncological breast surgery. Springerplus 2014, 3:517.

7. Bouman EA, Theunissen M, Bons SA, van Mook WN, Gramke HF, van Kleef M, Marcus MA: Reduced incidence of chronic postsurgical pain after epidural analgesia for abdominal surgery. Pain Pract 2014, 14(2):E76-84.

8. Stessel B, Ovink JK, Theunissen HM, Kessels AG, Marcus MA, Gramke H: Is sketamine with or without magnesium sulphate an alternative for postoperative pain treatment? Randomised study. Eur J Anaesthesiol 2013, 30(2):91-93.

9. Maas E, Meer vd F, Theunissen HMS, Marcus MAE: Urapidil, een vergeten medicijn? NTVA 2013, 26(2):3-5. 
10. Lance MD, Henskens YM, Nelemans P, Theunissen MH, Oerle RV, Spronk HM, Marcus MA: Do blood collection methods influence whole-blood platelet function analysis? Platelets 2013, 24(4):275-281.

11. Theunissen M, Peters ML, Bruce J, Gramke HF, Marcus MA: Preoperative anxiety and catastrophizing: a systematic review and meta-analysis of the association with chronic postsurgical pain. Clin J Pain 2012, 28(9):819-841.

12. Lance MD, Marcus MA, van Oerle R, Theunissen HM, Henskens YM: Platelet concentrate transport in pneumatic tube systems - does it work? Vox Sang 2012, 103(1):79-82.

13. Veldhorst-Janssen NM, Fiddelers AA, van der Kuy PH, Theunissen HM, de Krom MC, Neef C, Marcus MA: Pharmacokinetics and tolerability of nasal versus intravenous midazolam in healthy Dutch volunteers: a single-dose, randomized-sequence, openlabel, 2-period crossover pilot study. Clin Ther 2011, 33(12):2022-2028.

14. Severdija E, Heijmans J, Theunissen M, Maessen J, Roekaerts P, Weerwind P: Retrograde autologous priming reduces transfusion requirements in coronary artery bypass surgery. Perfusion 2011, 26(4):315-321.

15. Veldhorst-Janssen NML, Fiddelers A.A., Kuy van der P-H.M., Kessels A.G.H., Theunissen H.M.S., Hulst van der R.W.J., Neef C., Marcus M.A.E.: Pharmacokinetics, Analgesic Effect, and Tolerability of a Single Preprocedural Dose of Intranasal Fentanyl in Patients Undergoing Drain Removal after Breast Reduction or Augmentation Surgery: A Prospective, Randomized, Double-Blind, Placebo-Controlled Study. Clin Ther 2010, 32(7):1427-1436.

16. Theunissen HMS: Referaat "A randomized, controlled trial of oxycodone versus placebo in patients with postherpetic neuralgia and painful diabetic neuropathy treated with pregabalin" (Zin C.S. et al., 2009). In: Nederlands Tijdschrift voor Pijn en Pijnbestrijding. the Netherlands: Nederlandse Vereniging ter Bestudering van Pijn; 2010: 18.

17. Heijmans JH, Ganushak YM, Theunissen MS, Maessen JG, Roekaerts PJ: Predictors of cardiac responsiveness to fluid therapy after cardiac surgery. Acta Anaesthesiol Belg 2010, 61(3):151-158.

18. Theunissen HMS: Referaat "Disrupted sleep in the night before breast surgery is associated with increased postoperative pain (Wright C.E. et al., 2009)". In: Nederlands Tijdschrift voor Pijn en Pijnbestrijding. vol. 28. the Netherlands: Nederlandse Vereniging ter Bestudering van Pijn; 2009: 30-31.

19. Schnabel RM, Marcus MA, Theunissen HM, Janssens MM: Anesthetic management for a child with mitochondrial complex II deficiency (Letter to editor). Paediatr Anaesth 2008, 18(8):802-803. 
20. NVA\&NVOG: Richtlijn medicamenteuze pijnbehandeling tijdens de bevalling. In.: Kwaliteitsinstituut voor de Gezondheidszorg CBO; 2008: 141.

21. Bouman EAG, H. F. Wetzel, N. Vanderbroeck, T. H. Bruinsma, R. Theunissen, M. Kerkkamp, H. E. Marcus, M. A. : Evaluation of two different epidural catheters in clinical practice. Narrowing down the incidence of paresthesia! Acta Anaesthesiol Belg 2007, 58(2):101-105.

\section{MANUSCRIPT ACCEPTED FOR PUBLICATION}

1. Stessel B, Theunissen M, Marcus MAE, Joosten EAJ, van Kuijk SM, Fiddelers AA, Peters ML, Hoofwijk D, Buhre WF, Gramke H-F: Prevalence and predictors of patient non-adherence to pharmacological acute pain therapy at home after day surgery: a prospective cohort study. Pain Pract 2017.

\section{POSTER PRESENTATIONS}

1. Theunissen M, Peters ML, Jonker S, Gramke H-F, Marcus MAE: Validity of the Surgical Fear Questionnaire in cataract patients. In: The 8th World Congress of the World Institute of Pain. New York, USA; 2016.

2. Theunissen M, Gramke H-F, Marcus MAE, Peters ML: Predictors of pain 12 months after hysterectomy. In: 9th Congress of the European Pain Federation EFIC. Vienna, Austria; 2015.

3. de Waal B, Theunissen M, Gramke H, Marcus M, Peters M: Predictors of acute pain after hysterectomy In: IASP 15th World Congres on Pain. Buenos Aires, Argentina; 2014.

4. Oversier N, Theunissen M, Gramke H-F, Marcus M, Peters M: Validation of the Surgical Fear Questionnaire in adult patients waiting for elective day-case surgery. In: IASP 14th World Congress on Pain. Milan, Italy; 2012.

5. Timmer VC, Theunissen HM, Peters ML, Gramke H-F, Marcus MA: Preoperative anxiety and catastrophizing: a systematic review of the association with chronic post surgical pain. In: Joint Annual Scientific Meeting 2011 BPS \& CPS. Edinburgh, UK; 2011.

6. Theunissen M, Bouman E, Peters M, Gramke H-F, van Kleef M, Marcus M: Chronic post-surgical pain: Is there a role for epidural anesthesia and analgesia in the prevention? . In: NVA Wetenschapsdag. Ede, the Netherlands; 2009. 
7. Bouman E, Theunissen M, Kessels A, Marcus M, Gramke H-F: Paravertebral block for analgesia after major oncologic breast surgery: a comparison of continuous paravertebral block, single shot paravertebral block, and single shot wound infiltration. In: NVA Wetenschapsdag. Ede, the Netherlands; 2009.

8. Theunissen M, Bouman E, van Saet A, Gramke H-F, Marcus M: Thoracic epidural anesthesia: comparison of 2 different catheters. In: XXVII ANNUAL ESRA CONGRESS Genoa, Italy; 2008. 
